\title{
SNARE assembly and regulation on membranes
}

\author{
PhD Thesis
}

\begin{abstract}
in partial fulfilment of the requirements
for the degree "Doctor of Philosophy"

in the Molecular Biology Program

at the Georg August University Göttingen,

Faculty of Biology
\end{abstract}

submitted by

Tabrez Jamal Siddiqui

born in

Calcutta, India 
I hereby declare that the Ph.D. thesis entitled "SNARE assembly and regulation on membranes" has been written independently and with no other sources and aids than quoted.

Tabrez Jamal Siddiqui 


\section{Acknowledgements}

I am grateful to my mentor and supervisor, Professor Reinhard Jahn, for giving me the opportunity to perform my Ph.D. in his laboratory and for his constant support and guidance.

I am thankful to Dr Dirk Fasshauer for his involvement in my work. His advice and support were instrumental during my Ph.D.

My deep gratitude is due to Dr Gottfried Mieskes for his enthusiasm and willingness to help with any problem that I encountered in the lab.

I am beholden to the following colleagues for their help and collaborations, without which this work would not be in its present form.

Dr Rainer Heintzman, presently at King's College, London, taught me Kinetic simulations.

Dr Silvio O. Rizzoli collaborated with me in setting up functional assays. He also performed the experiments involving direct imaging of isolated organelles. I am thankful to him for his help and advice whenever needed.

Ms Michaela Hellwig raised the monoclonal antibodies directed against the ternary SNARE complex. I deeply appreciate her readiness to help and assist at all times.

Dr Thorsten Lang collaborated with me in the experiments involving plasma membrane sheets. The membrane sheet assay involving BHK cells were done entirely by him.

Ms Ulrike Winter performed the disassembly experiments.

Dr Dietmar Riedel performed, from time to time, negative staining electron microscopy of liposomes and synaptic vesicles.

Dr Henning Urlaub performed the mass-spectrometry experiments.

Dr Shigeo Takamori taught me the synaptic vesicle enrichment protocol.

I am thankful to all my colleagues who helped in my experiments, among them $\mathrm{Dr}$ Christina Schütte, Dr Konark Mukherjee, Dr Mathew Holt, Dr Constanze Riel, Mr Alexander Stein, and Mr Christian Stegmann.

I am appreciative of my colleagues for an enjoyable experience in the lab and my friends in Göttingen for a positive social life.

I am thankful to my thesis committee members Professor Erwin Neher and Professor Nils Brose for their time and support.

I am indebted to Dr Steffen Burkhardt, the co-ordinator of the International Molecular Biology Program for making life easier from the beginning of my stay in Göttingen.

I look to my parents Mr Jamaluddin A. Siddiqui and Mrs Rehana Siddiqui with deep fondness and gratitude for their constant support and prayers.

I wish to thank my fiancée, Ms Nirmala Padmanabhan, for being by my side and encouraging me through all moments of despair and success. 


\section{Table of Contents}

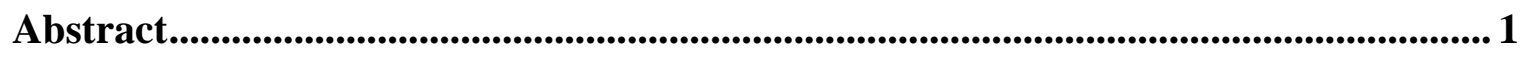

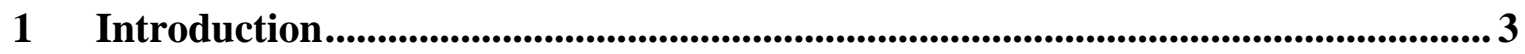

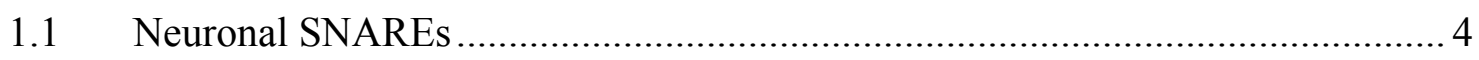

1.2 SNARE Assembly ........................................................................................ 7

1.2.1 Intermediates in the assembly pathway of SNAREs .............................. 8

1.2.2 N-terminal zippering model of SNARE complex assembly ...................... 9

1.3 Regulation of SNARE-mediated membrane fusion......................................... 11

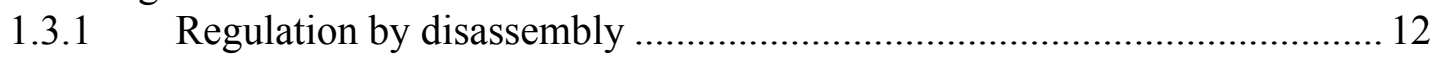

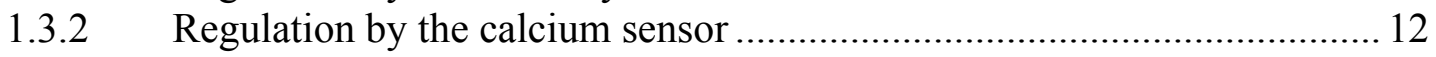



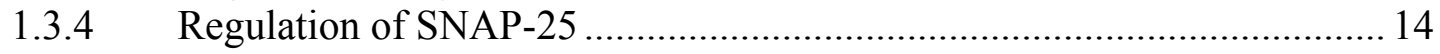

$1.4 \quad$ Emerging role of synaptobrevin in regulation ............................................... 14

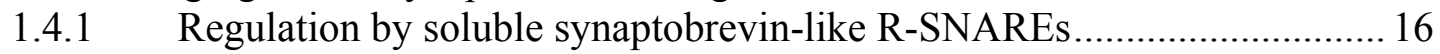

1.4.2 The synaptophysin/synaptobrevin heterodimer ...................................... 16



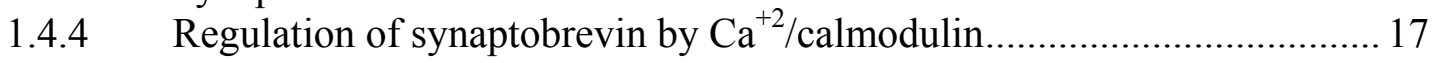

1.4.5 Regulation of synaptobrevin by the membrane ..................................... 18

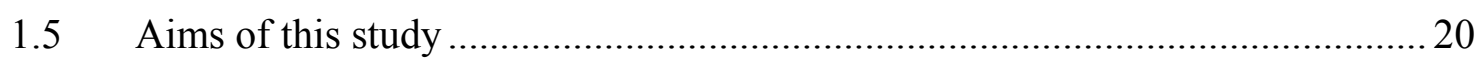

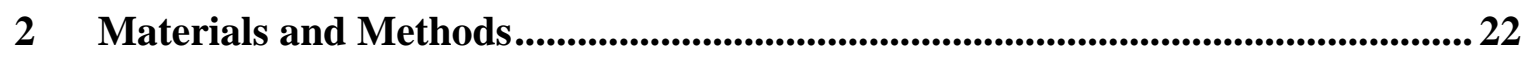

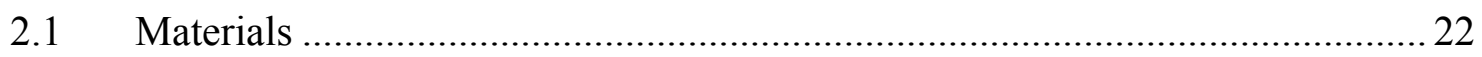

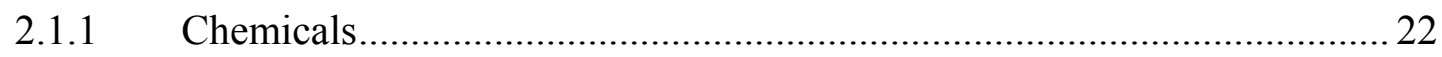

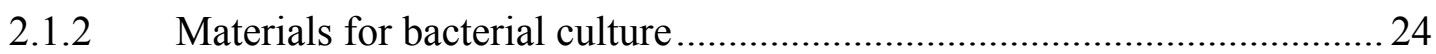

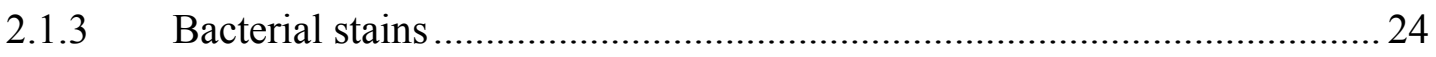

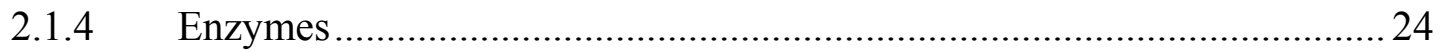

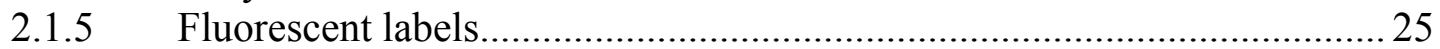

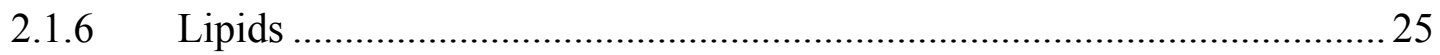

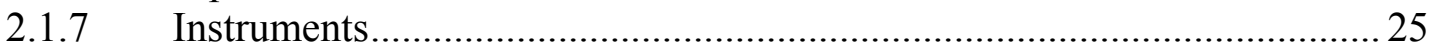

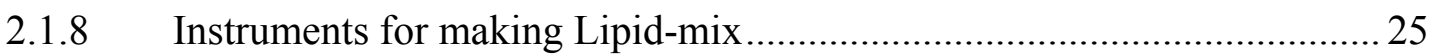

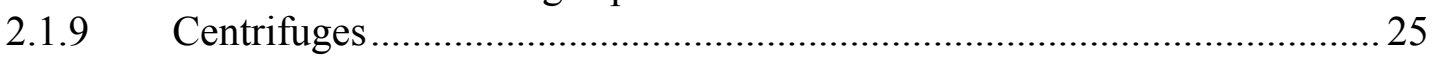



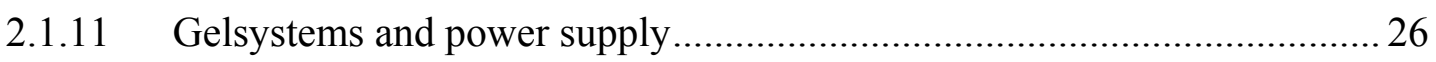



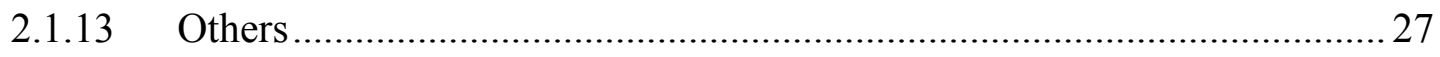

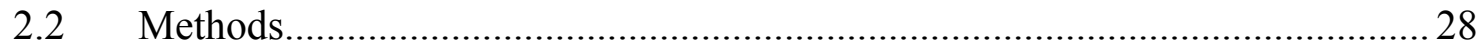

2.2.1 Protein Constructs and Molecular Cloning ............................................. 28

2.2.2 Protein Expression and Purification........................................................ 29

2.2.3 Determination of Protein Concentration .................................................. 31

2.2.4 Protein Labeling with Fluorescent Dyes................................................. 31

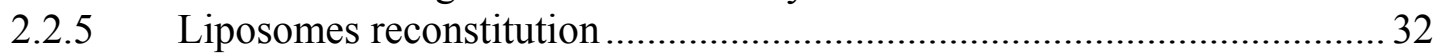

2.2.6 Digestion of proteoliposomes with toxins to determine protein orientation..

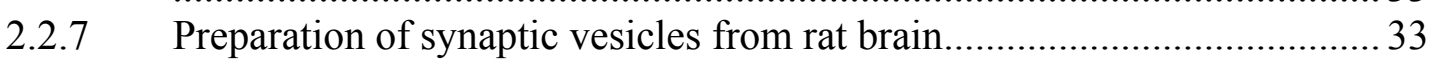


2.2.8 Preparing and running SDS-PAGE gels (Laemmli, 1970) ...................... 34

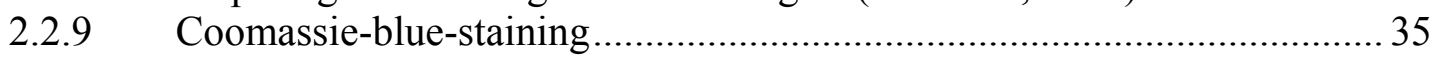



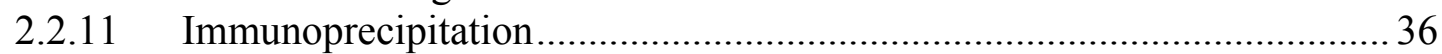

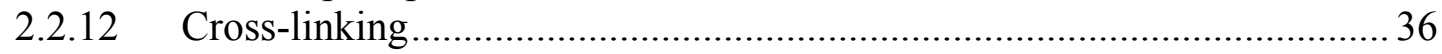

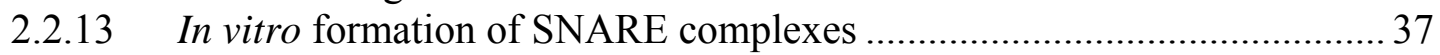

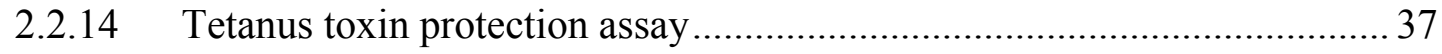

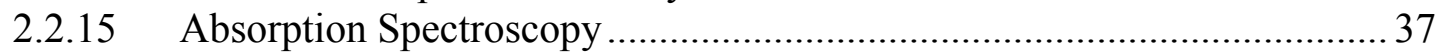

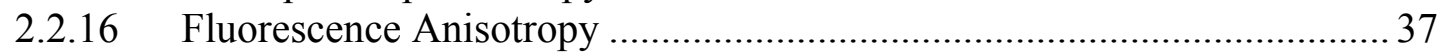

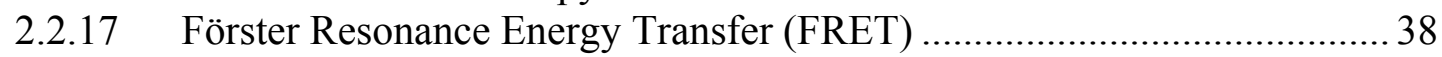

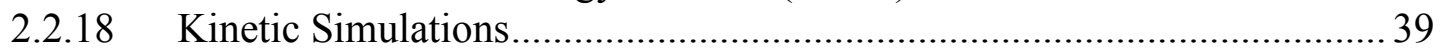

2.2.19 Antibody binding to ternary complex on synaptic vesicles ....................... 39

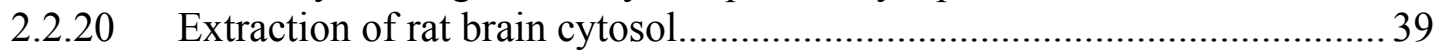

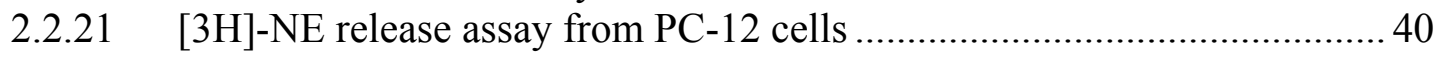



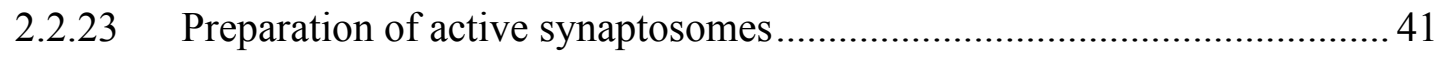

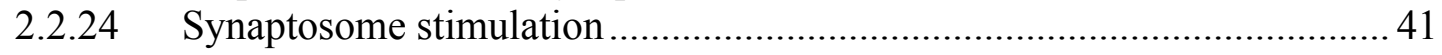

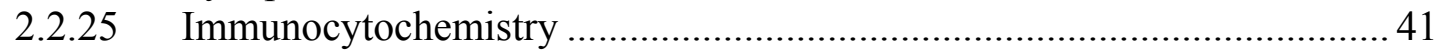



2.2.27 Direct labeling of synaptic vesicles ...................................................... 42

2.2.28 Mass-spectrometry ........................................................................ 42

2.2.29 Generation, immuonostaining, image acquisition and data analysis of

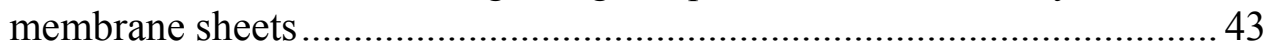

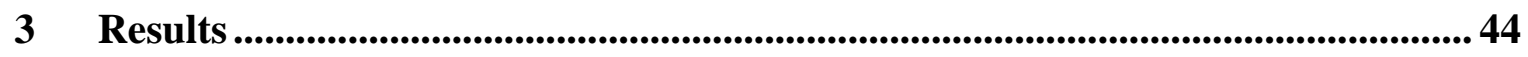

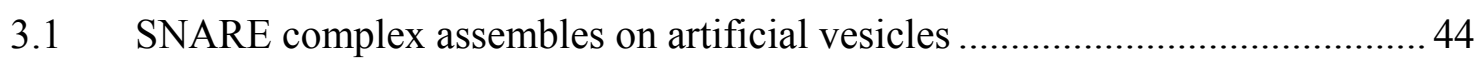

3.1.1 Liposomes do not influence assembly of soluble domains of SNAREs.... 45

3.1.2 SNARE complex assembles on syntaxin proteoliposomes ....................... 46

3.1.3 SNARE complex assembles on synaptobrevin proteoliposomes .............. 47

3.1.4 Fluorescence spectroscopy assays report kinetics of SNARE complex formation on artificial vesicles............................................................. 49

3.1.5 Reconstituted synaptobrevin engages SNAP-25 and syntaxin with the intact N-terminal domain in a complex .................................................... 54

3.1.6 Synaptobrevin in aged or frequently frozen/thawed liposomes becomes refractory to SNARE complex assembly ............................................ 55

3.2 Kinetics of SNARE complex assembly on artificial vesicles ...........................56

3.2.1 Assembly of SNARE complex on membranes proceeds via the syntaxin/

SNAP-25 binary complex ................................................................. 57

3.2.2 Kinetic Modelling of SNARE complex assembly on membranes............. 60

3.2.3 Substitution of membrane-proximal tryptophan residues by serine does not enhance the rate of complex formation................................................... 67

3.2.4 SNARE complex assembly rate is enhanced in the presence of weak

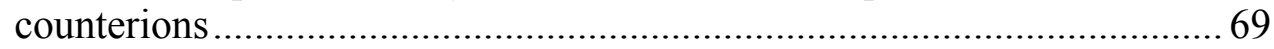

3.3 SNARE complex assembly on synaptic vesicles........................................... 73

3.3.1 SNARE complex formation on synaptic vesicles is not influenced by



3.3.2 Reactivity of synaptobrevin in liposomes and synaptic vesicles is comparable 
3.4 Soluble syntaxin and SNAP-25 displace synaptobrevin from the synaptophysin/synaptobrevin heterodimer to form the ternary SNARE complex 79

3.5 Characterisation of monoclonal antibodies against neuronal ternary SNARE complex.

3.6 The anti-ternary complex antibodies block disassembly of SNARE complexes by NSF/ $\alpha$-SNAP

3.7 The anti-ternary complex antibodies do not prevent release from permeabilized PC-12 cells

3.8 Preliminary results suggest that the total amount SNARE complex in a resting cell and stimulated cell is comparable

3.9 SNARE complexes are present on synaptic vesicles ..................................... 96



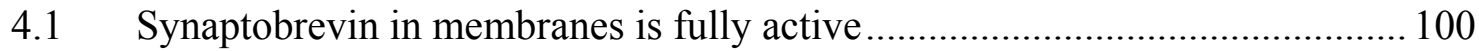

4.2 Role of membrane-proximal tryptophan residues of synaptobrevin............... 102

4.3 Synaptobrevin readily dissociates from synaptophysin to form SNARE complex

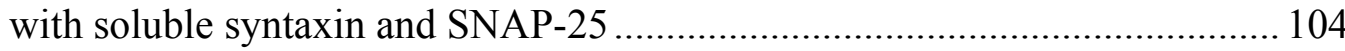

4.4 Kinetic modelling of SNARE complex assembly ......................................... 105

4.5 Anti-ternary complex antibodies: new tools for studying SNARE complexes 109

4.6 SNARE complexes dissociate immediately after exocytosis

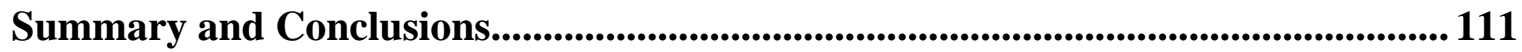

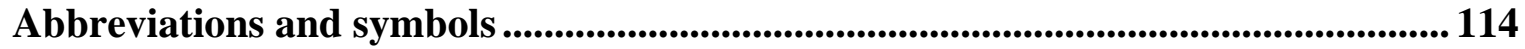

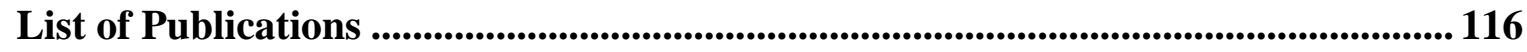

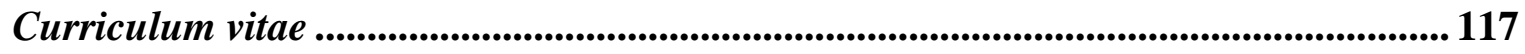

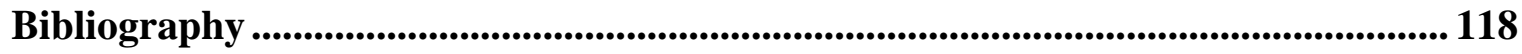




\section{List of Figures}

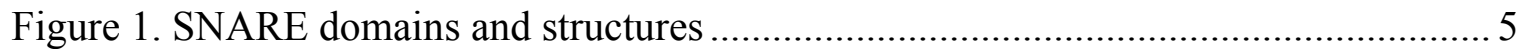

Figure 2. Putative model for SNARE mediated membrane fusion................................... 10

Figure 3. Status of synaptobrevin in membrane when not engaged in SNARE complex. Synaptobrevin (blue) may exist in one or more configurations illustrated above... .15

Figure 4. SNARE complex formation using soluble domains of SNAREs, monitored by SDS-PAGE and Coomassie Blue staining.

Figure 5. SNARE complex formation of soluble synaptobrevin (aa 1-96), syntaxin (aa 180-262) and SNAP-25 in the presence or absence of protein free liposomes. ........ 45

Figure 6. SNARE complex formation on syntaxin (Syx-) liposomes monitored by SDS-

PAGE followed fluorescence detection or Coomassie staining. ................................ 46

Figure 7. Orientation of synaptobrevin reconstituted in liposomes..................................... 48

Figure 8. SNARE complex formation on synaptobrevin (Syb)-containing liposomes, monitored by SDS-PAGE and Coomassie Blue staining. ……………………......... 48

Figure 9. SNARE complex formation on synaptobrevin (Syb)-containing liposomes monitored by SDS-PAGE and fluorescence anisotropy ……………………….....51

Figure 10. SNARE complex formation on synaptobrevin (Syb)-containing liposomes, monitored by FRET between synaptobrevin and SNAP-25

Figure 11. SNARE complex formation on synaptobrevin (Syb)-containing liposomes, monitored by FRET between synaptobrevin and syntaxin

Figure 12. SNARE complex formation on synaptobrevin (Syb)-containing liposomes with SNAP-25 and syntaxin (aa 1-262), i.e., syntaxin with the N-terminal Habc domain intact

Figure 13. Synaptobrevin in liposomes becomes refractory to SNARE complex assembly after prolonged storage or frequent freeze-thaw cycles............................................. 56

Figure 14. SNARE complex formation on synaptobrevin (Syb)-containing liposomes in the presence of detergent proceeds only moderately faster.....................................57

Figure 15. Increasing concentrations of SNAP-25 profoundly accelerates the rate of complex formation.

Figure 16. Simulation followed by fitting of the kinetic traces obtained in the SNAP-25 titration experiment, (FRET between $\mathrm{Syb}^{61 \mathrm{OG}} / \mathrm{Syx}^{225 T R}$ ).

Figure 17. Simulation followed by fitting of the kinetic traces obtained in the SNAP-25 titration experiment (FRET between Syb610G/Syx225TR).

Figure 18. Simulation followed by fitting of the kinetic traces obtained in the SNAP-25 titration experiment (FRET between Syb28OG/Syx197TR).

Figure 19. Substitution of the membrane proximal tryptophans 89 and 90 with serine does not alter the efficiency of synaptobrevin to enter SNARE complexes.

Figure 20. Rate of SNARE complex formation on synaptobrevin-containing liposomes is not changed by brain cytosolic proteins.

Figure 21. Rate of SNARE complex assembly does not depend on extraneous conditions.

Figure 22. SNARE complex formation on synaptobrevin (Syb)-containing liposomes with a lipid content of POPC:DOPS in a ratio of 85:15, monitored by FRET................ 71

Figure 23. Rate of SNARE complex assembly on synaptobrevin-containing liposomes in the presence of different solvents. ....................................................................... 73

Figure 24. SNARE complex assembly on synaptic vesicles (SV)................................... 75

Figure 25. SNARE complex assembly on synaptic vesicles does not change in the presence of increasing amounts of calcium. 
Figure 26. The rates of SNARE complex assembly on synaptic vesicles and on synaptobrevin liposomes are comparable.

Figure 27. Synaptobrevin is displaced from synaptophysin (Syp) upon formation of

SNARE complexes.

Figure 28. Binding specificity of anti-ternary complex antibodies to the SNARE complex.

Figure 29. Characterisation of the binding site of the novel monoclonal antibodies 135.1,

135.2 and 135.5

Figure 30. Immunoprecipitation followed by immunoblotting and mass-spectrometry of synaptopsome-enriched rat brain fraction.

Figure 31. Colocalization of ternary complex with membrane resident proteins,SNAP-25

(a) and syntaxin (b) and vesicular marker synaptophysin (c).

Figure 32. Supressing unspecific background of 135.1 staining on PFA-fixed membrane sheets.

Figure 33. Correlation of signals arising from incorporated fluorescently labelled synaptobrevin with anti-ternary complex antibody 135.1 immunostaining (a) or with anti-synaptobrevin antibody 69.1 immunostaining (b).

Figure 34. The anti-ternary complex antibodies can block disassembly of SNARE complexes by NSF/a-SNAP but does not abolish SNARE complex assembly, as assessed in a FRET assay....

Figure 35. $[3 \mathrm{H}]$ Norepinephrine secretion from Streptolysin-O permeabilized PC-12 cells.

Figure 36. Are ternary complexes dissociated before or after synaptic vesicle endocytosis?

Figure 37. A large fraction of synaptic vesicles binds anti-ternary complex antibodies 97

Figure 38. Sequence alignment of C-terminal residues of synaptobrevin homologs. ..... 103

Figure 39. Putative models for synaptobrevin binding to the syntaxin/SNAP-25 acceptor complex. 


\section{Abstract}

The fusion of synaptic vesicles with the pre-synaptic plasma membrane is mediated by SNARE proteins. This work provides key insights into the behaviour of synaptobrevin, the SNARE protein localized to synaptic vesicles, in its native membrane organelle and when it is reconstituted in liposomes. The reactivity of reconstituted synaptobrevin has remained controversial. Recent studies have suggested that synaptobrevin inserted in membranes does not readily engage in SNARE complexes $(\mathrm{Hu}$ et al., 2002; Kweon et al., 2003b). I therefore explored the binding characteristics and assembly pathway of the SNAREs on synaptobrevin-bearing membranes. Like its soluble domain, synaptobrevin anchored in membranes binds to the syntaxin/SNAP-25 acceptor complex to form the stable tetra helical coiled coil SNARE bundle. Kinetic simulations and fitting of experimental data helped unravel the SNARE assembly pathway on membranes. Monitoring the effects of endogenous and extraneous factors on SNARE complex assembly suggested that assembly is a robust process, largely unaffected by brain cytosolic factors, membrane fluidity, chaotropicity, divalent ions and lipid composition but is considerably enhanced in the presence of weak counter-ions. In agreement with these observations, SNARE complex assembly rate on liposomes reconstituted with synaptobrevin and on synaptic vesicles was comparable.

Being highly reactive molecules, the regulation of SNAREs has considerable importance. Synaptobrevin has been found to be associated in membranes with synaptophysin, an abundant protein localized to the synaptic vesicle and a potential regulator of SNARE complex assembly. I showed here that syntaxin/SNAP-25 binding to synaptobrevin in synaptic vesicles causes its dissociation from synaptophysin.

Three monoclonal antibodies recognizing the ternary SNARE complex, but not the monomers, were successfully raised and characterised in immunoblots, cell-lines and plasma-membrane sheets. Their binding sites on the complex were mapped. In a functional assay, the antibodies abolished disassembly of the SNARE complex thus providing insights into the disassembly machinery.

The antibodies can be used in other functional assays to answer pertinent questions. The status and dynamics of SNARE complexes was probed in pre-synaptic nerve-terminals. I found that the amount of SNARE complex is comparable in resting and stimulated synaptosomes, suggesting that they rapidly disassemble immediately after exocytosis has occurred. Some amount of complex was, however, found on isolated 
organelles when monitored by direct imaging or immunoblotting, suggesting that rapid disassembly of the SNARE complex is not a pre-requisite for endocytosis, though it cannot be ruled out that complexes formed on isolated organelles in vitro. 


\section{Introduction}

The predominant mechanism of information transfer between neurons occurs via the release of neurotransmitters at specialised sites called chemical synapses (Loewi, 1921). A typical chemical synapse is defined by a pre- and a post-synaptic neuron separated by a 20-40 nm gap called the synaptic cleft (Kandel, 2000). Upon the arrival of an action potential and subsequent elevation of intracellular calcium concentration in the pre-synaptic nerve terminal, specialised organelles called synaptic vesicles laden with neurotransmitters fuse with the plasma membrane and release their content into the synaptic cleft (Katz, 1969). Neurotransmitters diffuse across the synaptic cleft and bind to post-synaptic receptors, thereby eliciting specific post-synaptic responses, reviewed in (Sheng and Kim, 2002).

The merger of synaptic vesicles with the presynaptic plasma membrane is a temporally - occuring at sub-millisecond time-scale - and spatially regulated process (Rettig and Neher, 2002; Rosenmund et al., 2003). Synaptic vesicles are targeted to specialised electron dense sites called 'active zones' on the presynaptic plasma membrane (Akert, 1971) and primed into a 'release-ready' state prior to calcium entry into the presynaptic bouton (Rettig and Neher, 2002). Fusion occurs by membrane contact, merger, and the opening of an aqueous fusion pore (Jahn et al., 2003). The merging of two membranes involves their deformation and proceeds via low-energy intermediates (Kuzmin et al., 2001) which may be overcome by fluctuations and perturbations in the local lipid environment (Jahn and Grubmuller, 2002). Concomitant with activitydependent exocytosis, spontaneous fusion or miniature release events (minis) have been observed in neurons (Katz and Thesleff, 1957). In all biological fusion reactions, proteins have been suggested to bring into close apposition two disparate membranes and by their perturbation, thought to catalyze fusion (Jahn and Grubmuller, 2002). Such a role has been suggested for the SNARE proteins (Hanson et al., 1997; Lin and Scheller, 1997).

SNARE proteins mediate all vesicular fusion events within the cell, with a few notable exceptions, among them that of miochondria (Griffin et al., 2006), sperm-egg fusion (Inoue et al., 2005) and virus entry into host cell, reviewed in (Jahn et al., 2003). Insights into the involvement of SNAREs in membrane trafficking in yeast led to the view that intracellular fusion events require engagement of SNAREs in transport vesicles (vSNAREs) and target membranes (t-SNAREs), reviewed in (Jahn and Sudhof, 1999). The 
functional importance of synaptic SNAREs was first demonstrated in studies showing them to be targets of clostridial neurotoxins (Blasi et al., 1993; Blasi, 1993; Heiner Niemann, 1994; Link et al., 1992; Schiavo et al., 1992), which block neuronal exocytosis. Mutational studies in Drosophila (Broadie, 1995; Fergestad et al., 2001; Schulze et al., 1995), C. elegans (Nonet et al., 1998; Richmond et al., 2001) and mice (Schoch et al., 2001; Washbourne et al., 2002) further demonstrated the significance of synaptic SNAREs in evoked release. Furthermore, SNAREs reconstituted in liposomes were able to fuse them (Schuette et al., 2004; Tucker et al., 2004; Weber et al., 1998), suggesting that SNAREs comprise the minimal fusion machinery.

\subsection{Neuronal SNAREs}

SNAREs are characterised by 60-70 amino acids arranged in heptad repeats, termed SNARE motifs, which have a high tendency to form coiled coils (Fasshauer et al., 1997b). The SNAREs involved in neuronal exocytosis are syntaxin 1, synaptobrevin 2 and SNAP-25 (Fig. 1a). These three proteins assemble spontaneously into a complex that sediments at $7 \mathrm{~S}$ (Sollner et al., 1993a).

Synaptobrevin or VAMP (Vesicle associated membrane protein) is a $12.5 \mathrm{kDa}$ (116 amino acids) type 2 membrane protein, confined to synaptic vesicles (Baumert, 1989 ; Trimble et al., 1988). Two isoforms, synaptobrevin 1 and synaptobrevin 2 are known to be differentially expressed in the nervous system (Raptis et al., 2005). In this work, synaptobrevin 2 shall be referred to as simply synaptobrevin. Synaptobrevin has a short unstructured N-terminal region (Fig. 1a), (Hazzard et al., 1999) which, in its homologs, is either absent or replaced by a folded N-terminal profilin-like domain referred to as the longin domain (Gonzalez et al., 2001; Tochio et al., 2001). The cytoplasmic domain contains one SNARE motif which was determined to be unstructured in solution by circular dichroism (Fasshauer et al., 1997b) and solution state NMR studies (Hazzard et al., 1999).

Syntaxin 1, a $33 \mathrm{kDa}$ (288 amino acids) type 2 membrane protein, is localized to the pre-synaptic plasma membrane (Bennett, 1992 ). Two isoforms, syntaxin 1a and syntaxin $1 \mathrm{~b}$ with $84 \%$ sequence homology are differentially expressed in the central and peripheral nervous systems (Ruiz-Montasell, 1996 ). In this work, syntaxin 1a shall be referred to as simply syntaxin. Syntaxin contains a well-structured N-terminal three helix 
bundle called the $\mathrm{H}_{\mathrm{abc}}$ domain (aa 24-150) linked to a single SNARE motif by a flexible linker (Margittai et al., 2003a). X-ray and NMR structures of the $\mathrm{H}_{\mathrm{abc}}$ domain suggest that it is well structured with three alpha helices (Fernandez et al., 1998; Lerman et al., 2000) (Fig 1a). The groove between the second and the third helix is conserved. Though unstructured in solution, the SNARE motif of syntaxin tends to adopt a homotetrameric structure at very high concentrations (Misura et al., 2001b).

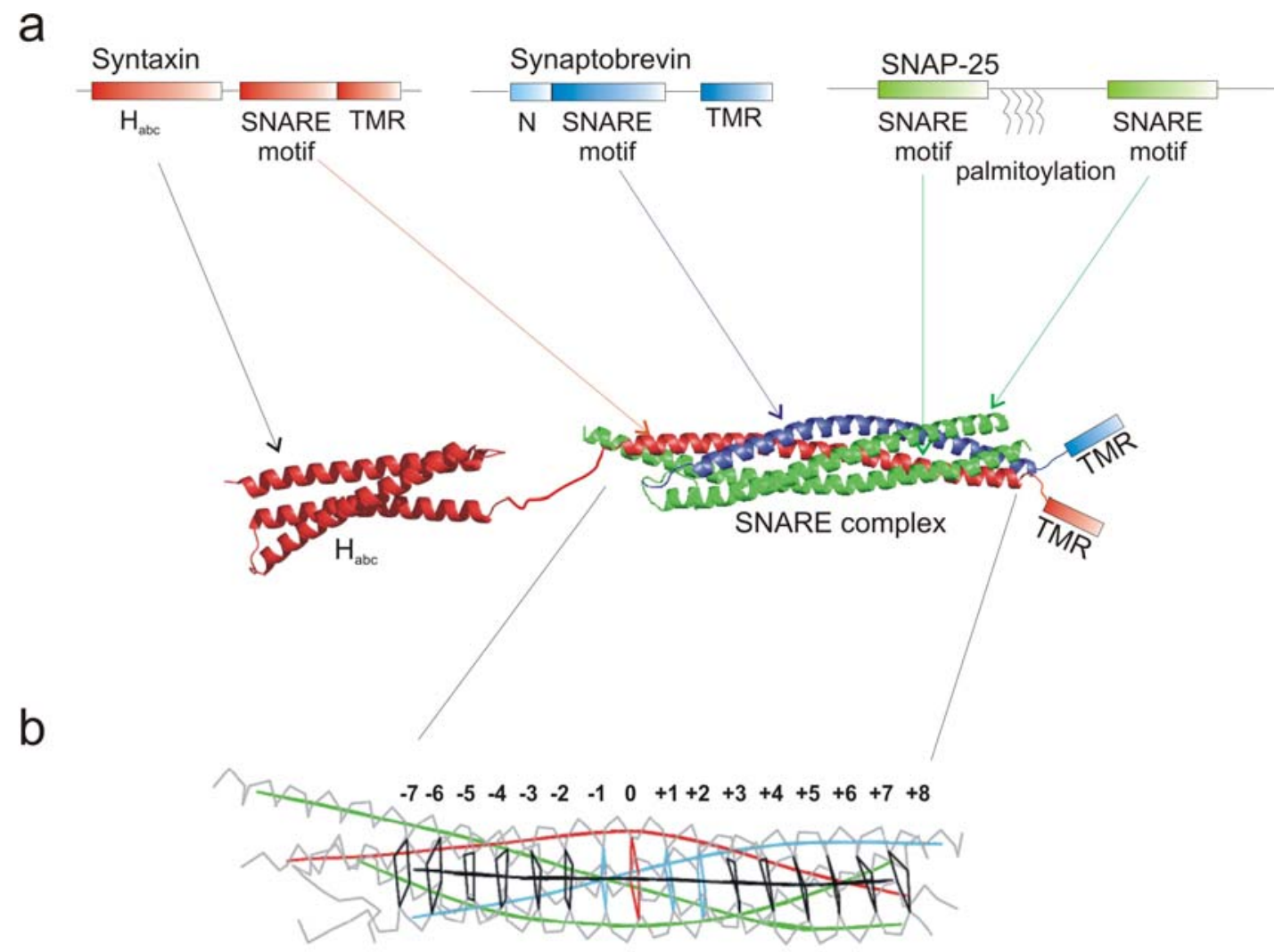

Figure 1. SNARE domains and structures

a) The amino terminal domain of syntaxin $\left(\mathrm{H}_{\mathrm{abc}}\right)$ forms a three helical bundle (PDB 1EZ3); (Lerman et al., 2000). The SNARE motifs of syntaxin, synaptobrevin and SNAP-25 form a four helical coiled coil structure (PDB 1SFC);(Sutton et al., 1998). Synaptobrevin contains an N-terminal proline-rich amino terminal $(\mathrm{N})$. Notice that whereas both syntaxin and synaptobrevin contain a trans-membrane region (TMR), SNAP-25 attaches to the membrane through the palmitoylation anchors at the four cysteine residues in the central domain between the two SNARE motifs.

b) Schematic drawing of the neuronal SNARE complex showing the layers -7 to $+8 ; 0$ layer in red, $-1,+1,+2$ in blue and all the other layers in black. Fig. $b$ was reproduced from (Sutton et al., 1998). 
SNAP-25 (synaptosome-associated protein of $25 \mathrm{kDa}, 206$ amino acids) (Oyler et al., 1989) contains two SNARE motifs which are associated with the presynaptic plasma membrane via palmitoyl chains on the linker between the SNARE motifs (Fig. 1a) (Hess et al., 1992). Two splice variants, SNAP-25a and SNAP-25b which differ in only nine amino acids are known (Bark et al., 1995). In this study, SNAP-25a shall be referred to as simply SNAP-25. Circular dichrosim (CD) experiments have shown that isolated SNAP25 is unstructured in solution (Fasshauer et al., 1997b).

In isolation, the SNAREs are mainly unstructured but upon contact with each other, their SNARE motifs spontaneously assemble in parallel into a twisted, tight, tetrahelical coiled coil bundle (ternary complex) of very high kinetic stability (Fig. 1a) (Fasshauer et al., 2002; Fasshauer et al., 1997b; Sutton et al., 1998). Whereas syntaxin and synaptobrevin contribute one helix each to the ternary complex, SNAP-25 contributes two helices. Four mainly hydrophobic residues, one from each of the SNARE motifs interact at sixteen positions in the interior of the structure (Sutton et al., 1998). These positions are referred to as layers numbered from -7 to +8 (Fig. 1b). The helix bundle is largely stabilised by these hydrophobic interactions. The 0-layer, unlike the other layers is not comprised of hydrophobic residues; instead, three glutamines (Q), contributed by syntaxin and SNAP-25 and one arginine (R), contributed by synaptobrevin interact to form a polar layer. The polar layer co-ordinates a $\mathrm{H}_{2} \mathrm{O}$ molecule (Brunger and Ernst, 2002). The 3Q:1R topology is conserved in other SNARE complexes (Antonin et al., 2002b; Pobbati et al., 2004). Based on these observations, the SNAREs were reclassified as Q- and R- SNAREs (Fasshauer et al., 1998b). The Q-SNAREs were further classified as $\mathrm{Q}_{\mathrm{a}^{-}}, \mathrm{Q}_{\mathrm{b}^{-}}$and $\mathrm{Q}_{\mathrm{c}^{-}}$SNAREs (Bock et al., 2001). Syntaxin homologs were classified as $\mathrm{Q}_{\mathrm{a}}$-SNAREs, and SNAP-25 helix 1 and helix 2 homologs were classified as $\mathrm{Q}_{\mathrm{b}^{-}}$and $\mathrm{Q}_{\mathrm{c}^{-}}$ SNAREs respectively. It was proposed that a fusion competent SNARE complex must contain a $\mathrm{Q}_{\mathrm{a}}, \mathrm{Q}_{\mathrm{b}}, \mathrm{Q}_{\mathrm{c}}, \mathrm{R}$ combination (Bock et al., 2001; Fasshauer et al., 1998b). Mutagenesis studies have confirmed that a $3 \mathrm{Q}: 1 \mathrm{R}$ configuration is required for functional SNARE complexes (Graf et al., 2005; Katz and Brennwald, 2000; Ossig et al., 2000). The $\mathrm{Q}$ and $\mathrm{R}$ system of nomenclature has proven to be more accurate than the $\mathrm{t}$ - and $\mathrm{v}$ - system of nomenclature since many t-SNAREs are found in vesicles and v-SNAREs are found on target membranes (Cao and Barlowe, 2000; Liu and Barlowe, 2002). Likewise, the Q and $\mathrm{R}$ system is better suited to define homotypic vesicular fusion (Brandhorst et al., 2006). 


\subsection{SNARE Assembly}

The proposal that the energy released during SNARE complex formation is sufficient to induce fusion is supported by molecular dynamics simulation studies indicating that the energy of about $3-8 \mathrm{kcal} / \mathrm{mol}$ of unstructured SNAREs could be transduced cooperatively by the linker region of syntaxin (Knecht and Grubmuller, 2003) trespassing low-energy intermediates on way to fusion (Kuzmin et al., 2001). The extraordinary stability of the SNARE complex (Fasshauer et al., 2002) lends credence to the view that the energy of fusion is derived from SNARE complex formation. These findings, together with genetic (Broadie, 1995; Fergestad et al., 2001; Nonet et al., 1998; Richmond et al., 2001; Schoch et al., 2001; Schulze et al., 1995; Washbourne et al., 2002), biochemical (Blasi et al., 1993; Blasi, 1993; Heiner Niemann, 1994; Link et al., 1992; Schiavo et al., 1992; Schuette et al., 2004; Tucker et al., 2004; Weber et al., 1998) and physiological studies (Nagy et al., 2004; Sakaba et al., 2005; Sorensen et al., 2002; Wei et al., 2000; Xu et al., 1999) hint strongly at SNAREs being putative fusogens.

The $\mathrm{H}_{\mathrm{abc}}$ domain of syntaxin, folds back on its SNARE motif in what is known as the closed conformation of syntaxin (Chen and Scheller, 2001). This conformation is stabilised by a protein called Munc18-1 or nSec1, a key regulatory protein in neuronal exocytosis (Dulubova et al., 1999; Misura et al., 2000). The closed conformation of syntaxin reduces the interaction of its SNARE motif with that of synaptobrevin and SNAP-25 (Margittai et al., 2003b). In in vitro experiments, the removal of the $\mathrm{H}_{\mathrm{abc}}$ domain accelerates the rate of SNARE complex formation ten-fold (Margittai et al., 2003c). The $\mathrm{H}_{\mathrm{abc}}$ domain does not interact tightly with the SNARE complex as evinced by thermal denaturation experiments (Fasshauer et al., 1997b; Karin L. Nicholson, 1998; Rice et al., 1997). Furthermore, the linker between the $H_{a b c}$ domain and the SNARE motif of syntaxin was shown to be highly flexible when syntaxin was engaged in SNARE complexes (Margittai et al., 2003a). Moreover, in yeast, when the Q-SNARE complex is made intramolecular, the $\mathrm{H}_{\mathrm{abc}}$ domain of the yeast plasma membrane syntaxin, Ssolp, is not required for efficient SNARE complex formation (Van Komen et al., 2006). These findings suggest that once SNARE complexes have formed, the $\mathrm{H}_{\mathrm{abc}}$ domain acts independently of the tetra-helical SNARE complex bundle 


\subsubsection{Intermediates in the assembly pathway of SNAREs}

Synaptic SNAREs have been found to interact with each other in a variety of combinations. Only a subset of these interactions probably represents biologically relevant intermediates.

The binding affinity between syntaxin and SNAP-25 has been determined to be high (Pevsner et al., 1994), whereas that between syntaxin and synaptobrevin is weak (Calakos et al., 1994). Indeed, thermal denaturation of the SNARE complex followed by renaturation revealed that the assembly pathway of the ternary SNARE complex transits through a less stable intermediate (Fasshauer et al., 2002). This intermediate was found to be a binary complex between syntaxin and SNAP-25. The distinct hysteresis profile for SNARE diassembly and assembly follows from the fact that the system does not remain in thermodynamic equilibrium at any given time. Therefore free energy changes involved in SNARE complex assembly have not been possible to measure. The studies by Fasshauer and colleagues (Fasshauer et al., 2002) estimate the rate of SNARE complex disassembly to be as slow as $4 \times 10^{-18} \mathrm{~s}^{-1}$. Such a large kinetic barrier suggests that certain factors are required for disassembly so that the SNAREs can be reused in subsequent rounds of fusion. Indeed, several lines of evidence show NSF, a homo-hexameric protein complex belonging to the AAA+ family of ATPases, in conjuction with its cofactor $\alpha$ SNAP to be involved in the disassembly process (Horsnell et al., 2002; Littleton et al., 1998; Pallanck et al., 1995; Sollner et al., 1993a; Sollner et al., 1993b; Whiteheart et al., 1993).

In solution, syntaxin and SNAP-25 interact to assume a coiled coil domain structure, as assessed by the increase in alpha helicity in CD experiments (Fasshauer et al., 1997a). The stoichiometry of syntaxin and SNAP-25 in a complex was determined to be 2:1 in experiments comprising non-denaturing and denaturing PAGE (Fasshauer et al., 1997b).

The structure of the complex between syntaxin and SNAP-25 is a parallel four helix bundle like the ternary SNARE complex, with one of the syntaxins occupying the binding site for synaptobrevin (Margittai et al., 2001; Xiao et al., 2001). In addition to the 2:1 syntaxin/SNAP-25 heterodimer, the SNARE motif of syntaxin can form a homotetramer of two pairs of parallel helices arranged in an anti-parallel configuration (Misura et al., 2001b) and a four helix bundle with only the first half of SNAP-25 (Misura et al., 2001a). The complex between syntaxin and the first half of SNAP-25 was 
also observed in live endocrine cells using fluorescently tagged versions of the proteins (An and Almers, 2004). The biological significance of homo- and hetero- oligomeric forms of syntaxin is not known.

These off-pathways, however, do complicate studies of the bona fide assembly pathway of the ternary SNARE complex, which proceeds via a 1:1 syntaxin/SNAP-25 complex, an intermediate to which a synaptobrevin molecule can readily bind (Fasshauer and Margittai, 2004). The binding mode of synaptobrevin to the 1:1 syntaxin/SNAP-25 heterodimer has been difficult to assess due to the transient nature of this intermediate: the formation rate of the syntaxin/SNAP-25 1:1 dimer has been determined to be a second order rate constant of $6000 \mathrm{M}^{-1}-\mathrm{s}^{-1}$ (Fasshauer and Margittai, 2004) and $\mathrm{k}_{\text {off }}$ for this product is estimated to be $0.01 \mathrm{~s}^{-1}$ (Dirk Fasshauer, unpublished observations). The assembly of SNAREs has been suggested to commence at the N-terminal ends of the molecules - according to the so-called N-terminal zippering model (Hanson et al., 1997; Lin and Scheller, 1997).

\subsubsection{N-terminal zippering model of SNARE complex assembly}

Both synaptobrevin and syntaxin are type II membrane proteins and SNAP-25 is attached to the plasma membrane via its palmitoyl linkers. Hence it is conceivable that their 'zippering' from the N-terminal to the C-terminal ends would bring the synaptic vesicle in close proximity to the plasma membrane. The energy of complex assembly would then be transduced via the membrane proximal linker to the local lipid environment causing perturbations, consequently leading to fusion (Fig. 2) 


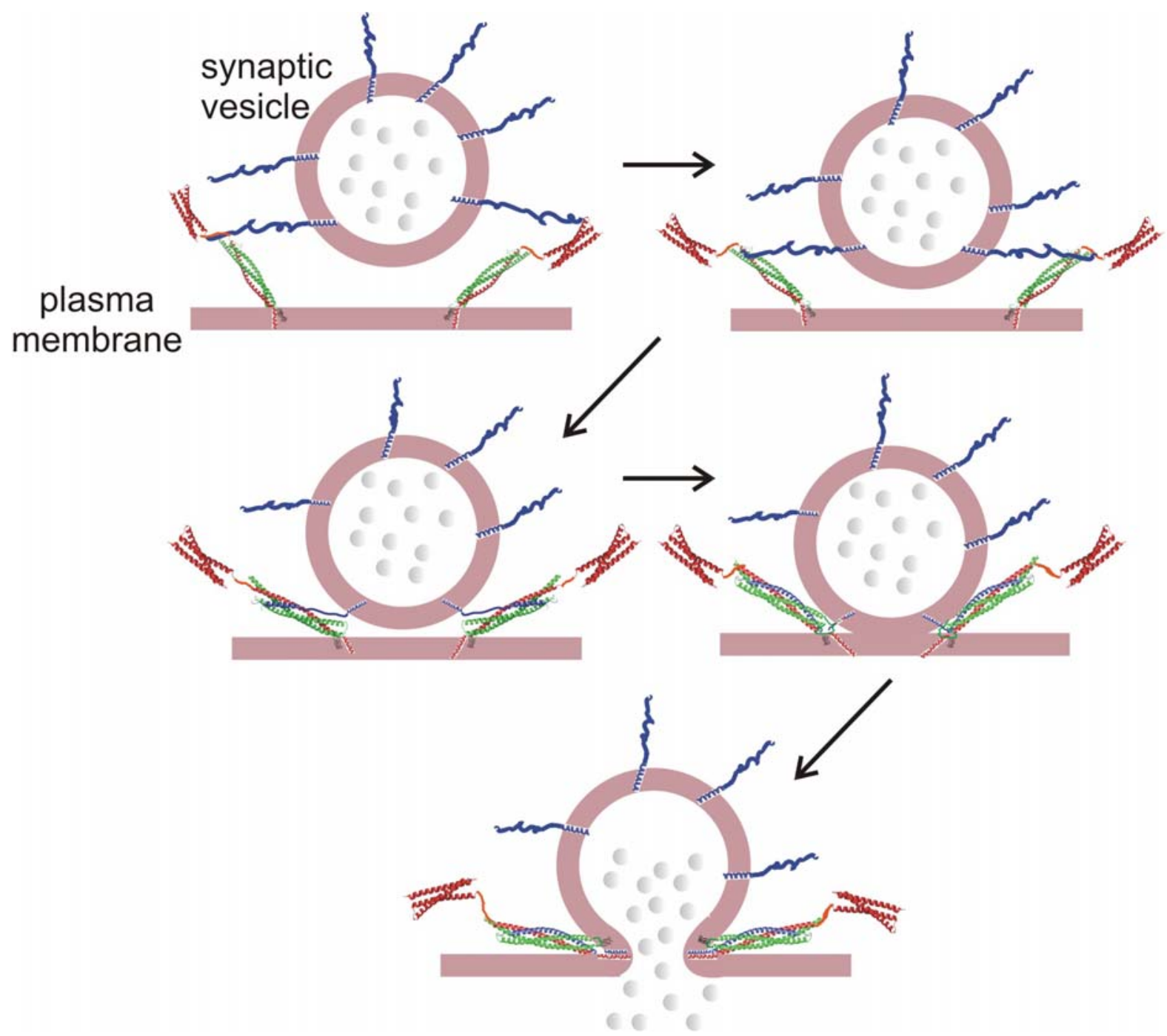

Figure 2. Putative model for SNARE mediated membrane fusion.

Syntaxin (red) and SNAP-25 (green) on the plasma membrane form an acceptor site for binding of synaptobrevin (blue). Binding of synaptobrevin to the syntaxin/SNAP-25 acceptor complex beginning at the $\mathrm{N}$-terminal end brings the neurotransmitter-laden synaptic vesicle and the plasma membranes in close apposition. The energy released in complex formation is transduced to the membranes, perturbing them and thereby causing fusion.

It has been suggested that a 1:1 heterodimer between syntaxin and SNAP-25 is first formed as an intermediate to which synaptobrevin binds and zippers from the N-terminal to the C-terminal end (Fasshauer and Margittai, 2004). Though attractive, this model has not been directly proven. Several indirect lines of evidence, however, exist for the model. First, the N-terminus of Sso1/Sec9p, yeast homolog of syntaxin/SNAP-25, is structured and provides an acceptor site for Snc1/2p, yeast homolog of synaptobrevin, which is suggested to bind to this N-terminus structured end and initiate zippering (Fiebig et al., 1999). Second, the presence of small polar residues like threonines and serines which are 
are in leucine zipper proteins but frequently occur in the SNARE motif may form intermolecular hydrogen bonds that could assist in directed assembly (Antonin et al., 2002b). Third, deuterium exchange on amides of synaptobrevin monitored by two dimensional NMR $\left({ }^{1} \mathrm{H}^{15} \mathrm{~N}\right.$ TROSY-HSQC) suggests that the $\mathrm{N}$-terminal half of the SNARE complex is more tightly packed than the C-terminal half (Chen et al., 2002). Fourth, in electrophysiological studies of adrenal chromaffin cells, a monoclonal antibody raised against the N-terminal end of SNAP-25 was shown to block complex formation (Xu et al., 1999). Fifth, fluorescence spectroscopy studies determined that whereas Cterminal truncations of syntaxin and SNAP-25 do not block assembly, the N-terminal truncations abolish assembly (Fasshauer and Margittai, 2004). Sixth, toxin cleavage analysis in crayfish neurons indicated that the N-terminal end of synaptobrevin is shielded in a complex (Hua et al., 1998). Seventh, studies involving mutation of SNAREs in cracked PC-12 cells suggest a sequential assembly of SNAREs starting from the Nterminal end (Chen et al., 2001).

Two modes of SNARE assembly starting from the N-terminal end are conceivable. One, once assembly starts, it zippers down all the way to the C-terminal end, transducing all the energy contained in the unfolded SNARE monomers to the lipids, causing fusion. This implies that the SNAREs would have to be prevented from making contacts before the calcium trigger to avoid undesirable fusion events. Two, assembly comes to a halt mid-way resulting in partially zippered complexes, probably stabilised by repulsive forces of the membranes or by other proteins. Upon calcium elevation, SNARE complex assembly would zipper to completion resulting in fusion. Either way, evoked neurotransmitter release is under the control of several auxiliary factors and hence intricately regulated.

\subsection{Regulation of SNARE-mediated membrane fusion}

A fundamental question in the field is how the highly reactive SNAREs are controlled to ensure that fusion occurs at a defined location and with defined kinetics. It is conceivable that the regulation of SNARE-mediated fusion of synaptic vesicles with plasma membranes may occur at any stage in the vesicle exo-endocytosis cycle. Though not known, it is possible that the level and activity of regulators is controlled by supraregulatory mechanisms, which would indirectly affect SNARE activity. Also, it cannot be ruled out that vesicle fusion is controlled by regulating the rate of disassembly of 
SNAREs by NSF/ $\alpha$-SNAP, thereby controlling the rate of vesicle recycling. The other possibilities are that SNAREs are directly acted upon by other factors. Two ways are conceivable in which SNAREs are directly controlled. One, the assembly of SNAREs may be blocked by a calcium sensor after partial zippering has occurred. An elevation of intracellular calcium would relieve this block causing fusion. Two, the SNAREs may be kept from interacting with each other by various factors thereby regulating the number of fusion-competent vesicles or number of sites on the active zone capable of supporting fusion. Some of the ways by which vesicle fusion may be regulated are discussed below.

\subsubsection{Regulation by disassembly}

SNARE complexes, being kinetically trapped (Fasshauer et al., 2002), need to be disassembled for reuse of the individual monomers. Co-sedimentation experiments revealed a $20 \mathrm{~S}$ complex comprising one SNARE complex, three alpha SNAPs and one hexameric NSF, (Wimmer et al., 2001). It is generally believed that NSF and its cofactor, $\alpha$-SNAP constitute the disassembly machinery (Chen and Scheller, 2001; Jahn et al., 2003). Drosophila with a temperature-sensitive allele for NSF undergo a slow onset of paralysis when they were subjected to heat shock at $38^{\circ} \mathrm{C}$ (Pallanck et al., 1995). This slow onset of paralysis is probably due to unavailability of free monomeric SNAREs for subsequent rounds of fusion. It is therefore conceivable, though not established, that under physiological conditions, the activity of NSF and/or $\alpha$-SNAP is altered to modulate the availability of free SNAREs.

\subsubsection{Regulation by the calcium sensor}

Neuro-exocytosis, being a calcium-mediated process, requires a calcium sensor. One of the original proposals was that this trigger is intrinsic to the SNARE complex. Though two sites on the surface of the SNARE complex formed by acidic and hydrophilic residues on SNAP-25 (D85/E170/Q177) and synaptobrevin (S75/E78/T79) were found to co-ordinate divalent ions in neuronal SNARE complex (Sutton et al., 1998), further evidence, including NMR and modelling studies, have discounted the direct binding and calcium-triggering role of SNARE complex (Chen et al., 2005; Rickman et al., 2004). In the search for a putative calcium sensor, much attention has shifted to a $65 \mathrm{kDa}$ protein called synaptotagmin 1 , discovered as a calcium binding protein localized to synaptic 
vesicles and large dense core vesicles, reviewed in (Chapman, 2002). Synaptotagmin binds to calcium via its $\mathrm{C} 2$ domains at an affinity at which neuro-exocytosis occurs (Brose et al., 1992; Davis et al., 1999). Mice carrying mutation in the synaptotagmin I gene have severely impaired neurotransmitter release and die within 48 hours of birth (Geppert et al., 1994). Synaptotagmin has been shown to bind to phospholipids in a calcium dependent manner (Brose et al., 1992; Shin et al., 2003) and to SNAREs in calcium-dependent and independent manners (Bai et al., 2004; Chapman et al., 1995; Rickman and Davletov, 2003). Various hypotheses exist on the mode of synaptotagmin action. One possibility is that it clamps SNARE complexes from fully zippering. Upon calcium influx, the block would be released, which would enable full SNARE pairing. Another possibility is that upon calcium elevation, synaptotagmin binds SNARE complex and phospholipids simultaneoulsy and mediates membrane fusion in concert with SNAREs. The exact mode of syaptotagmin action is yet to be elucidated.

\subsubsection{Regulation of Syntaxin}

As discussed above, syntaxin switches between its open and closed conformation. Single molecule fluorescence studies estimated the time for this switch to be $0.8 \mathrm{~ms}$ (Margittai et al., 2003b). Syntaxin, in its closed conformation, binds to Munc18-1 which therefore has been suggested to control the availability of syntaxin for SNARE complex formation (Dulubova et al., 1999). Another protein, Munc13-1, has been suggested to mediate the release of syntaxin from the Munc18-1 block (Betz et al., 1997; Sassa et al., 1999). Mice deficient in the gene for either Munc18-1 or Munc13-1 show complete block in neurotransmitter release (Augustin et al., 1999; Verhage et al., 2000). Though these findings directly confirm the indispensable role of Munc18-1 and Munc13-1 in neuronal exocytosis, they are hard to reconcile with the proposed inhibitory block of syntaxin by Munc18-1. Though Munc13-1 has been proposed to prime the SNAREs for fusion (Rosenmund et al., 2003), its interaction with calmodulin has been implicated in modulating short-term plasticity (Junge et al., 2004). A possible mechanism by which syntaxin activity could be modulated is by its $\mathrm{N}$-terminal $\mathrm{H}_{\mathrm{abc}}$ domain. But apart from the folding of this domain on to the syntaxin SNARE motif (Dulubova et al., 1999), no role for it has yet been proposed. 


\subsubsection{Regulation of SNAP-25}

As described above, SNAP-25 has four palmitoylation anchors in the linker between the two SNARE motifs. Plasma membrane targeting of SNAP-25 by the palmitoylation anchors increases its local concentration and is necessary for SNARE complex formation. (Koticha et al., 2002). It has been suggested that the local concentration of SNAP-25 on the plasma membrane is controlled by rounds of palmitoylation and depalmitoylation as SNAP-25 in PC-12 cells and neurites was suggested to be dynamically palmitoylated (Hess et al., 1992; Lane and Liu, 1997). Furthermore, phosphorylation and dephosphorylation of SNAP-25 at Thr138 and Ser187 was found to be necessary to modulate the number of vesicles in the release-ready state (Nagy et al., 2002; Nagy et al., 2004), adding another level of complexity in regulation.

\subsection{Emerging role of synaptobrevin in regulation}

Over the years, it has emerged that synaptobrevin, predominantly resident on the synaptic vesicles, is under exquisite control. There are on an average 60-70 synaptobrevin molecules on a single synaptic vesicle (Takamori unpublished observations). To orchestrate the reactivity of such a large number of synaptobrevin molecules, several modes of regulation have probably evolved. In this section, the possible ways in which synaptobrevin reactivity is modulated shall be discussed (Fig. 3). 

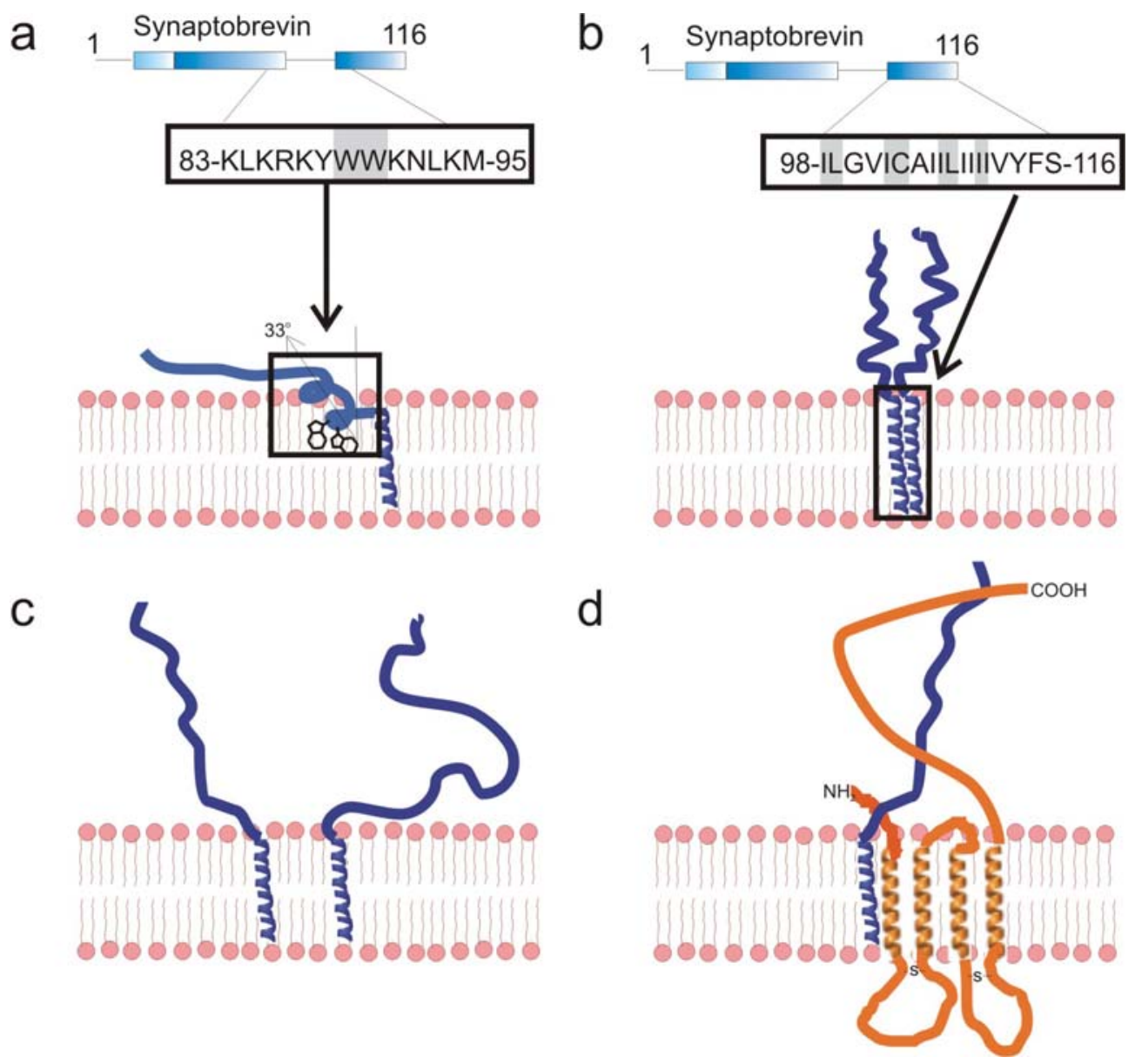

Figure 3. Status of synaptobrevin in membrane when not engaged in SNARE complex. Synaptobrevin (blue) may exist in one or more configurations illustrated above.

a) The membrane proximal aromatic residues tryptophan 89 and 90 dip into the membrane and drive residues 85-92 into an amphipathic helix (two $\alpha$-helical turns) at $33^{\circ}$ to the membrane normal. Based on EPR accessibility measurements, this model was proposed by (Kweon et al., 2003a; Kweon et al., 2003b).

b) Synaptobrevin dimerizes via its transmembrane regions. Based on site-directed mutagenesis experiments, Langosch and colleagues suggested the key eight residues (highlighted in the sequence) to be critical for synaptobrevin homodimerization (Laage and Langosch, 1997; Roy et al., 2004)

c) Synaptobrevin may not prefer any particular configuration in the membrane but remains largely unconstrained.

d) Synaptobrevin forms a labile heterodimer with synaptophysin 1 (Edelmann et al., 1995; Pennuto et al., 2002; Reisinger et al., 2004; Yelamanchili et al., 2005). This interaction may be largely mediated by the trans-membrane regions of both proteins (Edelmann et al., 1995). 


\subsubsection{Regulation by soluble synaptobrevin-like R-SNAREs}

Two soluble R-SNAREs have been found to be enriched in the brain: tomosyn and amisyn. Tomsyn is a $130 \mathrm{kDa}$ protein containing a C-terminal R-SNARE motif. It has been shown to form a tight ternary complex of very high kinetic stability with syntaxin and SNAP-25 (Hatsuzawa et al., 2003; Pobbati et al., 2004). The crystal structure of this complex was solved (Pobbati et al., 2004). It has a striking resemblance to the ternary complex with synaptobrevin. This complex can be disassembled by NSF and $\alpha$-SNAP (Hatsuzawa et al., 2003). In several studies, tomosyn was proposed to be a positive regulator of exocytosis (Baba et al., 2005; Fujita et al., 1998). Other studies have assigned a negative regulatory role for tomosyn suggesting that it directly competes with synaptobrevin to form the fusion-inactive tomosyn SNARE complex and thus act as a negative regulator of exocytosis (Hatsuzawa et al., 2003; Widberg et al., 2003; Yizhar et al., 2004). Amisyn is a $25 \mathrm{kDa}$ protein and like tomosyn, it contains a C-terminal RSNARE motif. Whereas the SNARE motif of amisyn does not efficiently inhibit exocytosis, full-length amisyn can block secretion of growth hormone from PC-12 cells (Constable et al., 2005; Scales et al., 2002). Although amisyn can form 'fusion incompetent' complexes with syntaxin and SNAP-25, its inhibitory role is not due to its binding to syntaxin (Constable et al., 2005).

\subsubsection{The synaptophysin/synaptobrevin heterodimer}

In the membrane of synaptic vesicles, synaptobrevin was shown to be associated with synaptophysin, a major multispanning membrane protein of synaptic vesicles (Fig 3d) (Calakos and Scheller, 1994; Edelmann et al., 1995; Washbourne et al., 1995). Synaptophysin 1 and its homolog synaptogyrin 1 together account for about $10 \%$ of vesicular proteins (Jahn et al., 1985; Wiedenmann and Franke, 1985). In transfected PC12 cells, synaptophysin 1 and synaptogyrin 1 are potent inhibitors of exocytosis (Sugita et al., 1999) indicating a role for these proteins in regulating exocytosis. Interaction of synaptobrevin with synaptophysin and syntaxin 1/SNAP-25 is mutually exclusive (Edelmann et al., 1995), suggesting that release of synaptobrevin from synaptophysin may constitute an (additional) activation step (Pennuto et al., 2002; Reisinger et al., 2004; Yelamanchili et al., 2005). However, genetic ablation of synaptophysin results in no detectable phenotype (McMahon et al., 1996). 
Mice lacking the gene for both synaptophysin 1 and its homolog synaptogyrin 1 have no visible phenotype but exhibit greatly attenuated long term potentiation (LTP) (Janz et al., 1999). Owing to the lack of visible phenotype in synaptophysin 1 or in both synaptophysin 1 and synaptogyrin 1 double knock-out mice, many researchers have questioned the functional role of these proteins in neuro-exocytosis. It is possible, though, that synaptophysin, via its interaction with synaptobrevin or another as yet unknown factor, may be involved in fine-tuning neuronal exocytosis under certain conditions. Further work needs to be done to elucidate the functional importance of such an abundant protein.

\subsubsection{Synaptobrevin dimerization}

Synaptobrevin can interact with different proteins with its different domains; whereas the SNARE motif interacts with syntaxin and SNAP-25 to form the ternary SNARE complex, the trans-membrane region has been suggested to interact with synaptophysin (Calakos and Scheller, 1994; Edelmann et al., 1995; Washbourne et al., 1995). Under denaturing conditions, a distinct dimeric form of synaptobrevin is discernible in SDS-PAGE (Fig. 3b) (Edelmann et al., 1995). Mutational and cross-linking studies suggested that synaptobrevin dimerizes via its trans-membrane domain (Laage and Langosch, 1997; Roy et al., 2004). The functional relevance, if any, for synaptobrevin dimerization, could be in the proposed multimerization of the SNARE complex (Laage et al., 2000) or in being a transitory species from the synaptophysin/synaptobrevin heterodimer to the SNARE complex. Another study, however, estimated the dissociation constant of synaptobrevin dimerization to be $10 \mathrm{mM}$, questioning the significance of this association in vivo (Bowen et al., 2002).

\subsubsection{Regulation of synaptobrevin by $\mathrm{Ca}^{+2} /$ calmodulin}

Initially calmodulin was suggested to be a candidate calcium sensor in neuroexocytosis (Burgoyne, 1984). However, the low off rate of $\mathrm{Ca}^{+2}$ dissociation from calmodulin cannot account for the transient nature of reponse to elevated calcium in nerve terminals (Teo and Wang, 1973). Another possible role suggested for $\mathrm{Ca}^{+2} /$ calmodulin is its regulation of synaptobrevin in the late stage of exocytosis (De Haro et al., 2003). $\mathrm{Ca}^{+2} /$ calmodulin has been reported to bind to synaptobrevin C-terminus (aa 77-90) with a $\mathrm{K}_{\mathrm{D}}$ of $0.7 \mu \mathrm{M}$ which is well below the intracellular calmodulin concentration of about 10 
$\mu \mathrm{M}$ (Quetglas et al., 2002). Synaptobrevin $77-90$ has also been suggested to bind to lipids (Quetglas et al., 2000). Using surface plasmon resonance spectroscopy (SPR) and epitope accessibility methods, it was suggested that $\mathrm{Ca}^{+2} /$ calmodulin transfers this domain of synaptobrevin from the cis- to trans- bilayer (de Haro et al., 2004). In a functional assay designed to monitor human growth hormone release from PC-12 cells, Ophiobolin A, a calmodulin antagonist, significantly blocked release (Quetglas et al., 2002), raising the stakes for calmodulin as a regulator. Nonetheless, calmodulin is involved in a variety of cellular processes and it cannot be ruled out that exocytosis was affected indirectly. Various other roles for calmodulin in regulating exocytosis or modulating short term plasticity have been suggested. (Brooks and Treml, 1984; Chamberlain et al., 1995; Junge et al., 2004; Okabe et al., 1992).

\subsubsection{Regulation of synaptobrevin by the membrane}

In recent years, several reports have suggested that membrane-inserted synaptobrevin is refractory to SNARE complex assembly (Hu et al., 2002; $\mathrm{Hu}$ et al., 2003; Kweon et al., 2003b). When isolated synaptic vesicles were incubated with recombinant SNAP-25 and syntaxin 1, no complex formation was observed. Synaptic vesicles did not fuse with liposomes reconstituted with syntaxin 1 and SNAP-25 unless calcium was added (Hu et al., 2002).

Synaptobrevin in synaptic vesicles was also unable to engage with soluble syntaxin and SNAP-25 in a complex in the presence of calcium. It was therefore suggested that synaptobrevin requires interaction with the calcium sensor, synaptotagmin I, for activation to catalyze fusion ( $\mathrm{Hu}$ et al., 2002). The reactivity of purified synaptobrevin was investigated after incorporation into liposomes by Electron paramagnetic resonance studies (Kweon et al., 2003b). No complexes formed with syntaxin 1 and SNAP-25, suggesting that synaptobrevin is intrinsically inactive when inserted into a bilayer. The lack of reactivity was attributed to the membrane-proximal region of synaptobrevin (aa 85-92) that is located adjacent to the transmembrane domain and includes the C-terminal end of the SNARE motif (Kweon et al., 2003b). This region was suggested to form an amphipathic helix that is tilted at an angle of $33^{\circ}$, with two conserved tryptophan residues (Trp89 and Trp90) dipping into the hydrophobic core of 
the bilayer (Fig 3a) (Kweon et al., 2003a). When these Trp-residues were replaced with serine, SNARE binding was restored (Kweon et al., 2003b). These authors suggested that SNARE assembly is directly regulated by the membrane, mediated primarily by the membrane-proximal tryptophan residues.

The data discussed above indicate that the SNARE motif of membrane-anchored synaptobrevin is normally inaccessible for complementary SNAREs, implying that synaptobrevin requires activation for fusion catalysis. However, synaptobrevin reconstituted in proteoliposomes readily fuses with liposomes containing syntaxin and SNAP-25 in a manner that is clearly dependent on the formation of SNARE complexes (Schuette et al., 2004; Tucker et al., 2004; Weber et al., 1998), a finding that is very difficult to reconcile with the notion that synaptobrevin is inaccessible in proteoliposomes. As discussed in section 1.2.2, in view of the widely believed N-terminal direction of SNARE assembly, the significance of the C-terminal restriction of synaptobrevin has remained somewhat elusive. 


\subsection{Aims of this study}

Chemical neurotransmission is an intricately regulated process. Synaptic vesicles filled with neurotransmitters fuse with the pre-synaptic plasma membrane with defined kinetics at specialised sites in the pre-synaptic nerve terminals called active zones. The most attractive candidates to execute fusion are the SNARE proteins (Chen and Scheller, 2001; Jahn et al., 2003; Rizo and Sudhof, 2002). The SNAREs, syntaxin and SNAP-25 on the plasma membrane and synaptobrevin in the synaptic vesicle, spontaneously assemble in solution to form stable tetra-helical coiled coils. Determining the factors that temporally and spatially regulate these highly reactive molecules would considerably enhance understanding of the molecular mechanisms underlying neurotransmission.

Synaptobrevin, the SNARE localized to the synaptic vesicles constitutes about $10 \%$ of the total vesicular protein pool and is the favourite substrate of clostridial neurotoxins. Botulinum toxins B, D, F, G and Tetanus toxin selectively cleave synaptobrevin, reviewed in (Breidenbach and Brunger, 2005). Understanding the mechanisms and pathways in which synaptobrevin is regulated is essential for enhancing knowledge of not just neuronal exocytosis but pathological conditions arising from clostridial neurotoxin poisoning. Extensive work on the assembly pathway of soluble domains of SNAREs suggests that synaptobrevin readily binds to the syntaxin/SNAP-25 acceptor complex (Fasshauer and Margittai, 2004). Is synaptobrevin in membrane as reactive as it is in solution? Several reports have suggested that reconstituted synaptobrevin is intrinsically inactive and cannot interact with syntaxin/SNAP-25 unless a membrane-solubilizing detergent is present (Chen et al., 2004; Kweon et al., 2003b). The first major goal of this work is therefore to ascertain whether reconstituted synaptobrevin is indeed refractory to SNARE complex formation.

If reconstituted synaptobrevin is able to form a complex, using kinetic traces from fluorescence spectroscopy measurements in combination with simulations and fitting of the data, I shall ask what the assembly pathway of the SNAREs is on membranes. The factors that may affect the kinetics of assembly shall be probed. Intrinsic factors like the interfacial residues suggested to cause membrane-insertion of the membrane-proximal region and effect of intracellular compoents shall be explored. Among the extraneous factors to be investigated shall be temperature, membrane fluidity, lipid composition, divalent ions, chaotropicity, electroconductivity and salt composition. 
Synaptobrevin in synaptic vesicles has also been suggested to be inhibited from engaging with syntaxin and SNAP-25 in a complex (Hu et al., 2002; Hu et al., 2004). I therefore intend to probe the reactivity of synaptobrevin in native vesicles and compare it to that of the reconstituted protein. In view of the report that synaptic vesicles fuse with syntaxin/SNAP-25 liposomes only in the presence of micromolar amounts of calcium, I ask if SNARE complex assembly on synaptic vesicles is influenced by calcium.

Synaptobrevin has been shown to be involved in a heterodimeric interaction with synaptophysin (Calakos and Scheller, 1994; Edelmann et al., 1995; Washbourne et al., 1995). This interaction is mutually exclusive to its interaction with syntaxin/SNAP-25 (Edelmann et al., 1995). A goal of this work is therefore to ask if synaptobrevin must first dissociate from synaptophysin to form the SNARE complex or whether the binding of syntaxin/SNAP-25 to synaptobrevin disrupts its interaction with synaptophysin.

Direct visualization of the SNARE complex within a cell without genetic manipulation has not been possible. I therefore intend to raise and characterise in immunoblots, cell-lines and plasma-membrane sheets, monoclonal antibodies that would recognise only the SNARE complex but not the individual monomers.

The next task shall be to determine whether the binding of the antibodies to the SNARE complex abolishes its disassembly by NSF/ $\alpha$-SNAP, which could form the basis of interesting and meaningful functional assays. I shall endeavour to determine the status and dynamics of SNARE complexes within a cell or pre-synaptic nerve-terminal. Among the parameters that I intend to investigate are subcellular localization of the complex, level of SNARE complexes in a resting cell and whether that level changes when stimulated for exocytosis. Finally, I would determine whether the antibodies can inhibit or potentiate release from permeabilized PC-12 cells, which would provide further insights into SNARE-mediated membrane fusion. 


\section{Materials and Methods}

\subsection{Materials}

\subsubsection{Chemicals}

Acetic acid, Merck (Darmstadt, Germany)

Acrylamide/Bisacrylamide solution, Roth (Karlsruhe, Germany)

Adenosine 5 '-triphosphate (ATP), Fluka (Switzerland)

Agarose (low EEO), Applichem (Darmstadt, Germany)

Albumin, bovine serum, Sigma (Deisenhofen, Germany)

Ammoniumpersulfate (APS), Sigma (Deisenhofen, Germany)

Ammonium Chloride, (Nagy et al.)

Ampicillin, Roth (Karlsruhe, Germany)

ß-Mercaptoethanol, Sigma (Deisenhofen, Germany)

BCIP, Boehringer (Mannheim, Germany)

Bromophenol blue, Sigma (Deisenhofen, Germany)

Bradford-Reagent, Biorad (Richmond, USA)

Calcium chloride dihydrate, Fluka (Switzerland)

Coomassie Brilliant Blue R-250, Serva (Heidelberg, Germany)

CHAPS, Sigma (Deisenhofen, Germany)

Cholate $3 \alpha 7 \alpha 12 \alpha$ Trihydroxy5 $\beta$ cholan 24oic acid sodium salt, Sigma (Deisenhofen, Germany)

Creatinine Kinase, Roche Diagnostics (Basel, Switzerland)

Creatinine Phosphate, Roche Diagnostics (Basel, Switzerland)

D (+)- Glucose, Sigma (Deisenhofen, Germany)

Dako Fluorescent mounting medium, Dako Diagnostika (Dako, Denmark)

Dithiothreitol (DTT), Roth (Karlsruhe, Germany)

Dipotassium hydrogen phosphate, Merck (Darmstadt, Germany)

Disodium hydrogen phosphate, Merck (Darmstadt, Germany)

DMF, Merck (Darmstadt, Germany)

Disuccinimydyl suberate (DSS), Pierce (USA)

EDTA Titriplex III, Merck (Darmstadt, Germany) 
EGTA, Sigma (Deisenhofen, Germany)

Ethanol, Merck (Darmstadt, Germany)

Glycine, Sigma (Deisenhofen, Germany)

Hepes-(2-hydroxyethyl)piperazine--N'-2-ethanesulphonicacid),GERBU(Gaiberg,

Germany)

Hydrochloric acid, Merck (Darmstadt, Germany)

Ionomycin, Merck (Darmstadt, Germany)

Imidazole, Sigma (Deisenhofen, Germany)

Isopropanol, Merck (Darmstadt, Germany)

Isopropyl- $\beta$-D-thiogalactoside (IPTG), Roth (Karlsruhe, Germany)

Kanamycin, Roth (Karlsruhe, Germany)

L-Glutamic acid monopotassium Sigma (Deisenhofen, Germany)

Magnesium chloride, Sigma-Aldrich (St.Louis, USA)

Methanol, Merck (Darmstadt, Germany)

N,N,N',N'- Tetramethylethylendiamine (TEMED), Biorad (Richmond, USA)

N-Ethylmaleimide (NEM), Sigma (Deisenhofen, Germany)

NBT, Boehringer (Mannheim, Germany)

Nycodenz, Nycomed Pharma from Axis-Shield Group (Oslo, Norway)

Octylglucoside, GERBU(Gaiberg, Germany)

Paraformaldehyde, Sigma-Aldrich (St.Louis, USA)

Poly-L-Lysine, Sigma (Deisenhofen, Germany)

Potassium acetate, Merck (Darmstadt, Germany)

Potassium dihydrogen phosphate, Merck (Darmstadt, Germany)

Potassium hydroxide, (Nagy et al.)

Potassium Iodide, (Nagy et al.)

PMSF (paramethyl sulphonyl fluoride), Roth (Karlsruhe, Germany)

Protease K, Boehringer (Mannheim, Germany)

Sodium chloride, Merck (Darmstadt, Germany)

Sodiumdodecylsulfate (SDS), Biorad (Richmond, USA)

Sodium hydrogen carbonate, Merck (Darmstadt, Germany)

Sucrose, Roth (Karslruhe, Germany)

Tricine, Applichem (Darmstadt, Germany)

Tris (hydroxymethyl)-aminomethane (Tris), Merck (Darmstadt, Germany) 
Triton X-100, Merck (Darmstadt, Germany)

Trypsin, Sigma (Deisenhofen, Germany)

Urea, Merck (Darmstadt, Germany)

\subsubsection{Materials for bacterial culture}

Tryptone, Roth (Karlsruhe, Germany)

Yeast extract, Roth (Karlsruhe, Germany)

Agar-agar, Roth (Karlsruhe, Germany)

LB (Luria Bertani) media: $10 \mathrm{~g}$ tryptone, $5 \mathrm{~g}$ yeast extract and $5 \mathrm{~g} \mathrm{NaCl}$, add 1 liter distilled water (added $12 \mathrm{~g}$ agar for solid media)

TB (Terrific broth) media: $13.3 \mathrm{~g}$ tryptone, $26.7 \mathrm{~g}$ yeast extract, $4.4 \mathrm{ml}$ glycerol.

TB salt: $0.17 \mathrm{M} \mathrm{KH}_{2} \mathrm{PO}_{4}, 0.72 \mathrm{M} \mathrm{K}_{2} \mathrm{HPO}_{4}$.

Before inoculation, the media were autoclaved and brought to room temperature. In the case of TB media, after autoclaving, TB salt was added.

\subsubsection{Bacterial stains}

The following strains were obtained from (Stratagene, La Jolla, CA, USA)

Escherichia coli BL21 (DE3) B F- dcm ompT hsdS (rB- mB-) gal $\lambda$ (DE3)

Escherichia coli XL-1-Blue recA1, endA1, gyrA96, thi-, hsdR17, (rk-, mk+), supE44, relA1, lac-, [F', traD36, proAB, lacIqZ $\Delta \mathrm{M} 15$, Tn10 (tetr)]

Escherichia coli JM109 e14-(McrA-) recA1 endA1 gyrA96 thi-1 hsdR17 (rK-mK+) supE44 relA1 $\Delta$ (lac-proAB) [F' traD36 proAB lacIqZ $\Delta \mathrm{M} 15]$.

Escherichia coli M15 strain contained additional pREP4 plasmid.

\subsubsection{Enzymes}

Restriction endonucleases, New England Biolabs (Ipswich, USA) or MBI Fermentas (Ontario, Canada)

Lyzozyme, Applichem (Darmstadt, Germany)

DnaseI, Applichem (Darmstadt, Germany)

Thrombin, Merck (Darmstadt, Germany) 


\subsubsection{Fluorescent labels}

Oregon Green ${ }^{\circledR} 488$ iodoacetamide, Invitrogen (USA)

Texas Red ${ }^{\circledR}$ C5 bromoacetamide, Invitrogen (USA)

Alexa Fluor ${ }^{\circledR} 594$ C5 maleimide, Invitrogen (USA)

\subsubsection{Lipids}

All lipids were purchased from Avanti Polar lipids (Alabaster, Al, USA)

Cholesterol (brain)

L- $\alpha$-Phosphatidylcholine (brain)

Phosphatidylethanolamine (brain)

Phosphatidylserine sodium salt (brain)

Phosphatidylinositol sodium salt (bovine liver)

1,2-Dioleoyl-sn-Glycero-3-(Pospho-L-Serine) (DOPS)

1-Palmitoy-2-Oleoyl-sn-Glycero-3-Phosphocholine (POPC)

\subsubsection{Instruments}

ÄKTA explorer (GE Healthcare)

Luminescent image analyzer, LAS-1000 Fujifilm (Japan)

Fluoromax-2, Horiba Jobin Yvon (Edison, NJ, USA)

Flurolog, Horiba Jobin Yvon (Edison, NJ, USA)

GeniosPro microplate fluorescence reader (Tecan)

UV-spectrophotometer, UV-2401 PC Shimadzu (Japan)

\subsubsection{Instruments for making Lipid-mix}

Rotavapor R-124, Büchi,

Vacuum Controller B-720, Büchi,

Waterbath B-480, Büchi,

\subsubsection{Centrifuges}

J6-MI, Beckman (Palo Alto, CA, USA)

Optima TL X Ultracentrifuge, Beckman (Palo Alto, CA, USA)

Optima L-90K Ultracentrifuge, Beckman (Palo Alto, CA, USA) 
RC 5C Plus centrifuge, Sorvall (Bad Homburg)

RC 5B Plus centrifuge, Sorvall (Bad Homburg)

\subsubsection{Rotors}

SLA-3000, (SuperLite ${ }^{\mathrm{TM}}$ GSA), Sorval, (Bad Homburg)

SLA-1500, (SuperLite ${ }^{\mathrm{TM}}$ GSA), Sorval, (Bad Homburg)

SW 28, Beckman (Palo Alto, CA, USA)

SW41, Beckman (Palo Alto, CA, USA)

SW50.1, Beckman (Palo Alto, CA, USA)

SS34, Sorvall, (Bad, Homburg)

TLA 55, Beckman (Palo Alto, CA, USA)

TLA 100.3, Beckman (Palo Alto, CA, USA)

TLA 120.2, Beckman (Palo Alto, CA, USA)

TLS 55, Beckman (Palo Alto, CA, USA)

Ti70, Beckman (Palo Alto, CA, USA)

Ti 50.2, Beckman (Palo Alto, CA, USA)

\subsubsection{Gelsystems and power supply}

Electrophoresis chamber Mini-Protean II, Biorad (Richmond, USA)

Geldryer Model 583, Biorad (Richmond, USA)

Power Pac 200, Biorad (Richmond, USA)

Power Pac 300, Biorad (Richmond, USA)

\subsubsection{Softwares}

Aida Image Analyzer, Raytest (Straubenhardt, Germany)

Corel Graphics suite 11, Corel Corporation

Endnote 9, Thomson

Kaleidagraph v4.03, Synergy software

Matlab 7.1, The MathWorks Inc. (Massachusetts, USA)

Pro-Kineticist II, Applied Photophysics, (Leatherhead, UK)

Pymol, DeLano Scientific LLC 


\subsubsection{Others}

G25-Sepahadex Beads, Pharmacia Biotech (Freiburg)

G50-Sephadex Beads, Pharmacia Biotech (Freiburg)

Gene Pulser II BioRad (Richmond, USA)

Microcon Centrifugal filter devices (Amicon)

Molecular weight protein standards, Sigma (Deisenhofen)

Molecular weight protein standards, MBI Fermentas

Ni-NTA-Superflow Beads, Qiagen (Hilden)

Protein-G Sepharose 4FastFlow (GE Healthcare)

Western Lightning Chemiluminiscence Kit, Perkin Elmer

DNA Isolation Kits, Machery Nagel (Düren, Germany) 


\subsection{Methods}

\subsubsection{Protein Constructs and Molecular Cloning}

All recombinant proteins were derived from cDNAs encoding for rat proteins and sub-cloned into pET28a, pET30, pET15, pHO2c or pGEX vectors (Novagen, Darmstadt, Germany) or pEQ3 (Qiagen, Hilden, Germany) which encode for either an aminoterminal His $_{6}$-tag or an N-terminal GST-tag. SNAP-25 (no cysteine), synaptobrevin (aa 196), syntaxin (aa 180-262), SN1 (SNAP-25A aa 1-83), the second helix of SNAP-25a., SN2 (SNAP-25A aa 120-206) (Fasshauer et al., 1998a), syntaxin(aa 1-288), syntaxin C225, syntaxin C197, SNAP-25 C84, SNAP-25 C130 (Margittai et al., 2001) and fulllength synaptobrevin (aa 1-116) (Margittai et al., 1999), syntaxin (aa 183-188) (Schuette et al., 2004), endobrevin SNARE motif (Fasshauer et al., 1999), syntaxin (aa 183-240), synaptobrevin (aa 1-81), synaptobrevin (aa 1-70), synaptobrevin (aa 25-96), synaptobrevin (aa 35-96), SNAP-25A, BoNT/A fragment (aa 1-197) (Fasshauer and Margittai, 2004), syntaxin (aa 1-262) (Margittai et al., 2003b), tomosyn SNARE-motif (Hatsuzawa et al., 2003) SNAP-23 (Wolfram Antonin), synaptobrevin (aa 1-76) (Margittai PhD Thesis) have been have been described previously. TeNT, BoNT C and BoNT A light chains, encoded in pEQ3 (Qiagen, Hilden) were gifts from H. Niemann (Medizinische Hochschule, Hannover, Germay). Cloning was performed according to standard protocols (Sambrook, 2001). Enzymes for DNA manipulations were obtained from New England Biolabs (Beverly, MA), and Ni-NTA agarose from Qiagen, oligonucleotides were obtained from MWG Biotech AG (Ebersberg, Germany). All other reagents were purchased from Sigma (Deisenhofen, Germany). Plasmid manipulations were performed using the Escherichia coli XL1-Blue strain. Single cysteines in the cytsoplasmic regions were introduced at positions 28, 61 and 79 of full-length synaptobrevin by site directed mutagenesis (Martin Margittai, unpublished work). Tryptophan residues at positions 89 and 90 of full-length synaptobrevin and of its respective single cysteine variants were mutagenized to serine residues using the forward primer: 5' GCT CAA GCG CAA ATA CTC GTC GAA AAA GCT CAA GAT GAT G 3' and reverse primer: 5' CAT CAT CTT GAG GTT TTT CGA CGA GTA TTT GCG CTT GAG C 3'. 
Syntaxin 2, syntaxin 3, syntaxin 4 and syntaxin 5 SNARE motifs were generated by a single PCR reaction. cDNAs encoding for rat syntaxin 2, syntaxin 3, syntaxin 4 and syntaxin 5 served as templates. The PCR products were cloned into their target vectors via an Nde1/Xho1 cleavage site. The cloned products, vector and primers are listed in following table.

\begin{tabular}{|c|c|l|}
\hline Construct & Vector & \multicolumn{1}{c|}{ Primer } \\
\hline Syx2 (aa 184-263) & pHO2c & $\begin{array}{l}\text { Start: 5'-GGAATTC CAT ATG TCA GAT TCA CAG ATT AC } \\
\text { Stop:5'-GCGCC CTC GAG TTA TCT GGC CTT GCT CTG G }\end{array}$ \\
\hline Syx3 (aa 183-261) & pHO2c & $\begin{array}{l}\text { Start: 5'-GGAATTC CAT ATG GAC TCC CAG ATT TCC AAG } \\
\text { Stop:5'-GCGGC CTC GAG CTA TCG AGC CTG ACC CTG }\end{array}$ \\
\hline Syx4 (aa 192-270) & pHO2c & $\begin{array}{l}\text { Start: 5'-GGAATTC CAT ATG GAC ACA CAG GTG ACC CG } \\
\text { Stop: 5'-GCGCC CTC GAG CTA CCT CGC CTT CTT CTG }\end{array}$ \\
\hline Syx5 (aa 201-279) & pGEX & $\begin{array}{l}\text { Start: 5'-GGAATTC CAT ATG CAG CTT CAG CTC ATT G } \\
\text { Stop: 5'-GCGCC CTC GAG TCA CCG ATT GGA GGT GAC }\end{array}$ \\
\hline
\end{tabular}

\subsubsection{Protein Expression and Purification}

All proteins, both cysteine free and cysteine containing constructs, were expressed in pET28a, pET30, pET15, pHO2c or pGEX vectors (Novagen, Darmstadt, Germany) in the BL21(DE3) strain of Escherichia coli containing either ampicillin or kanamycin resitance genes. Tetanus toxin light chain and BoNT C light chain, containing both kanamycin and ampicillin resistance genes, were encoded in pQE3 (Qiagen) in M15 strain of Escherichia coli. The proteins contained N-terminal His -tags $_{6}$ or GST-tags that were utilized to affinity purify them on nickel-nitrilotriacetic acid-agarose (Qiagen, Hilden) or sepharose beads coupled to glutathione (Qiagen, Hilden). The N-terminal tags can be cleaved off using the protease thrombin.

Transfection and expression of recombinant proteins were done according to (Dower et al., 1988). All plasmids containing the protein constructs were introduced into the relevant electro-competent E.coli strain by electroporation in Gene pulser II (Biorad) at $1.8 \mathrm{kV}, 200 \Omega$ and $25 \mu \mathrm{F}$ in an electroporation cuvette (diameter 1-2 mm, Biorad). The transformed bacteria was plated on Luria Broth (LB) agar plates containing 15\% agar and either $30 \mu \mathrm{g} / \mathrm{ml}$ kanamycin or $100 \mu \mathrm{g} / \mathrm{ml}$ ampicillin at $37^{\circ} \mathrm{C}$ for $14 \mathrm{~h}-16 \mathrm{~h}$. In the case of toxins, both kanamycin and ampicillin were used as antibiotics.

Bacteria were picked from single colonies and inoculated in $100 \mathrm{ml} \mathrm{LB}$ medium with kanamycin concentration of $0.3 \mathrm{mg} / 1$ or ampicillin concentration of $1 \mathrm{mg} / \mathrm{l}$ and 
incubated overnight at $37^{\circ} \mathrm{C}$ while shaking. $700 \mathrm{ml}$ TB medium containing $10 \% \mathrm{~TB}$ salt or LB medium with the appropriate antibiotic was inoculated with $10 \mathrm{ml}$ of the over-night culture and incubated at $37^{\circ} \mathrm{C}$ while shaking until the absorption at $600 \mathrm{~nm}$ reached $0.7-1$. The protein expression was induced with $1 \mathrm{mM}$ IPTG for 3 to 4 hours. The cultures were harvested by cenrtrifugation in 1 litre containers at $4,000 \mathrm{rpm}$ for 15 minutes in the Beckman J6-MI centrifuge. The harvested culture was resuspended in extraction buffer and incubated with lysozyme, DNAse and PMSF for 15 minutes at RT. Complete lysis was done by sonification ( 4 x 40 pulses, big tip, microtip limit). Bacterial debris was removed by centrifugation in an SLA-1500 or SLA-3000 rotor (Sorvall) (30 min at $16,000 \mathrm{rpm})$. Ni-beads or GST-Sepharose suspension was incubated with the supernatant for $3-4 \mathrm{~h}$ at $6^{\circ} \mathrm{C}$. After several washes with washing buffer $(50 \mathrm{mM}$ Tris/ $\mathrm{HCl}, \mathrm{pH} 7.4,250$ $\mathrm{mM} \mathrm{NaCl}, 4 \mathrm{mM}$ Imidazole) for His-tagged proteins and (20 mM Tris, pH 7.4, $250 \mathrm{mM}$ $\mathrm{NaCl}, 1 \mathrm{mM}$ EDTA, $1 \mathrm{mM}$ DTT) for GST-tagged proteins, protein was eluted with elution buffer (50 mM Tris/HCl, pH 7.4, $250 \mathrm{mM} \mathrm{NaCl} 400 \mathrm{mM}$ Imidazol) for His-tagged proteins and (50 mM Tris, $\mathrm{pH}$ 7.4, $50 \mathrm{mM}$ Glutathione, $100 \mathrm{mM} \mathrm{NaCl}, 1 \mathrm{mM}$ EDTA, 1 mM DTT) for GST-tagged proteins. The tags were cleaved by thrombin $(1 \mathrm{U} / \mu \mathrm{l})$ during overnight dialysis (20 mM Tris, $\mathrm{pH} 7.4,50 \mathrm{mM} \mathrm{NaCl}, 1 \mathrm{mM}$ DTT) in a dialysis bag of 2$10 \mathrm{kDa}$ molecular weight cut-off (Spectra Por molecular porous membranes, Spectrum). Proteins with transmembrane region were not purified further. Their purity was determined by SDS-PAGE. Protein concentration was determined by Bradford test (Bradford, 1976) and the proteins were stored at $-80^{\circ} \mathrm{C}$.

Syntaxin (aa 183-288) tended to form inclusion bodies that remained in the pellet after centrifugation. After high speed centrifugation $(14500 \mathrm{~g})$, the pellet was partially resuspended in $200 \mathrm{ml}$ extraction buffer containing $6 \mathrm{M}$ Urea, $1.5 \%$ sodium cholate and 10 $\mathrm{mM}$ B-mercaptoethanol, sonicated and incubated at $6^{\circ} \mathrm{C}$ for $2 \frac{1 / 2}{2}$ hours. The solution was cleared by centrifugation and incubated with Ni-beads followed by the procedures decribed above.

Proteins without transmembrane regions were further purified on a Mono Q (eg. syntaxin and SNAP-25) or Mono S column (eg. synaptobrevin) using the Äkta Explorer (GE Healthcare). After loading, the proteins were eluted with a linear gradient of $\mathrm{NaCl}$ (20 mM Tris pH 7.4, $50 \mathrm{mM} \mathrm{NaCl}, 20 \mathrm{mM}, 1 \mathrm{mM}$ DTT and $20 \mathrm{mM}$ Tris $\mathrm{pH}$ 7.4, $1 \mathrm{M}$ $\mathrm{NaCl}, 1 \mathrm{mM}$ DTT ). $3 \mathrm{mM}$ (final concentration.) DTT was added to the eluates. The peak fractions were pooled and analyzed on SDS-PAGE. 


\subsubsection{Determination of Protein Concentration}

Protein concentration was determined according to Bradford (Bradford, 1976). A set of standards containing 1, 2, 3, 4, $5 \mu \mathrm{g}$ bovine serum albumin and the proteins were diluted in $200 \mu \mathrm{d} \mathrm{dH} 2 \mathrm{O}$ and then mixed with $800 \mu \mathrm{l}$ Bradford solution, blanked against a similar preparation of Bradford reagent containing no protein. After incubation for $5 \mathrm{~min}$ at RT the absorbance at $595 \mathrm{~nm}$ wavelength was measured using a photometer (Pharmcia Biotech Novaspec II). The slope and the intercept as well as the correlation coefficient of the standard curve could be determined using the appropriate functions in Microsoft Excel. The correlation coefficient of the standard curve was taken as a quality indicator of the measurement and was usually greater than 0.995 . The measured sample absorbance was multiplied with the slope and the product was added to the intercept value of the standard curve to obtain the protein concentration of sample.

\subsubsection{Protein Labeling with Fluorescent Dyes}

Proteins with single cysteines at specific sites were labeled with dyes that react with the sulfhydryl group of cysteine. Proteins were first dialysed in degassed buffer overnight followed by dialysis for two hours in degassed buffer saturated with $\mathrm{N}_{2}$. The fluorescent dyes, Oregon Green 488 iodoacetamide, or Texas Red C5 bromoacetamide were dissolved in DMF and incubated for $2 \mathrm{~h}$ at $6^{\circ} \mathrm{C}$ in a ten-fold molar excess to the proteins to ensure that all sulfhydryl groups of the cysteines conjugated with the dyes.<smiles>CC(=O)Nc1cc(C(=O)O)c(-c2c3cc(F)c(=O)cc-3oc3cc(O)c(F)cc23)c(C(=O)O)c1</smiles>

Oregon Green

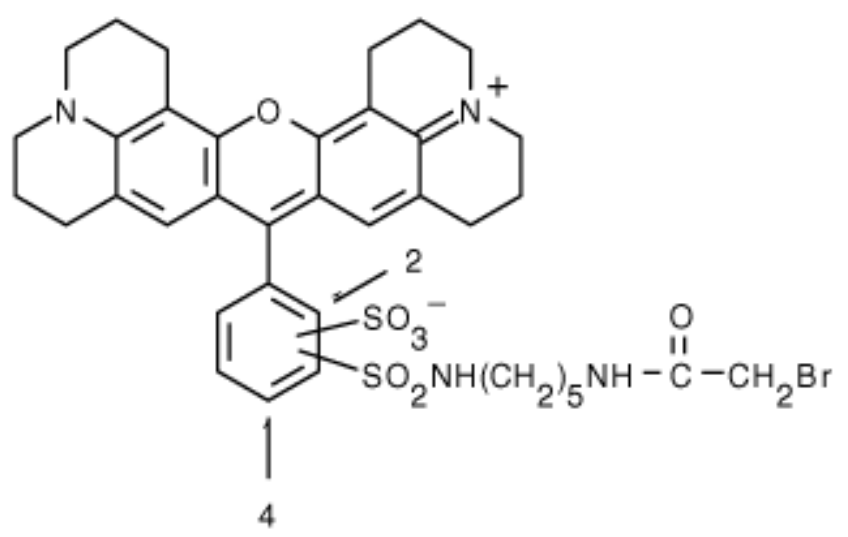

Texas Red 
The labelled proteins were applied on Biorad EconoColumn containing G-25 sephadex beads followed by extensive dialysis (PBS, $20 \mathrm{mM} \mathrm{Na} \mathrm{HPO}_{4}, \mathrm{pH}$ 7.4, 150 $\mathrm{NaCl}$ ) to remove unbound dye. The proteins were run on 15\% SDS gels and documented with an LAS-1000 reader (Fujifilm) using the appropriate filter sets (HQ470/40x (Cy2) Exiter / HQ525/50m (Cy2) Emitter, and HQ545/30x (Cy3) Exiter / HQ610/75m (Cy3) Emitter). All labeled proteins were more than 95\% pure and free of unincorporated label. Wherever needed, the proteins were concentrated in Microcons (YM-10).

\subsubsection{Liposomes reconstitution}

Lyophylized lipids (Avanti Polar Lipids) were solubilized in chloroform/methanol in a 2:1 (V/v) ratio immediately before use. Phosphatidylcholine, phosphatidylethanolamine, phosphatidylserine, phosphatidylinositol and cholesterol were mixed in a ratio of 5:2:1:1:1 under Argon. Alternatively, for the generation of liposomes with neutral lipids, phosphatidylcholine and cholesterol were mixed in a ratio of 7:3 under Argon. A third lipid mix of 1-Palmitoy-2-Oleoyl-sn-Glycero-3-Phosphocholine (POPC): 1,2Dioleoyl-sn-Glycero-3-Pospho-L-Serine (DOPS) in a ratio of 85:15 was prepared under argon. The lipid mixtures were dried on a rotary evaporator and resuspended in cholate buffer $(20 \mathrm{mM}$ Tris $\mathrm{pH} 7.4,120 \mathrm{mM} \mathrm{NaCl}, 1 \mathrm{mM} \mathrm{DTT}, 5 \%$ cholate $(\mathrm{w} / \mathrm{v}))$ at a detergent to lipid molar ratio of $8: 1$ and a final lipid concentration of $13.5 \mathrm{mM}$. Protein and the lipid-micellar mix containing 5\% cholate were mixed in a molar ratio of 1:100 with the volume of the lipid micellar mix being $100 \mu \mathrm{l}$. If the concentration of the protein was considerably low, it was concentrated in the Centricon MWCO 10.000. The protein-lipid mix was incubated at $4^{\circ} \mathrm{C}$ for 30 min followed by size exclusion chromatography on Biorad Econo Column of $0.7 \mathrm{~cm}$ diameter and $15 \mathrm{~cm}$ length packed with Sephadex G-50 (superfine) equilibrated in PBS or Hepes-buffered K-Glut (20 mM Hepes-KOH, pH 7.2, $120 \mathrm{mM}$ potassium glutamate, $20 \mathrm{mM}$ potassium acetate and 1mM EDTA). For the removal of unincorporated proteins, $500 \mu 1$ of the liposome fraction was mixed with an equal volume of $80 \%$ Nycodenz in buffer, overlaid with $500 \mu \mathrm{l}$ of $30 \%$ Nycodenz and 150 $\mu \mathrm{l}$ of buffer. The gradient was centrifuged at 165,000 $\mathrm{g}$ for $4 \mathrm{~h}$ in Beckman Ultra Clear 11 x $34 \mathrm{~mm}$ centrifuge in TLS 55 rotor. Liposomes were retrieved from the top of the gradient. A typical preparation yields $300 \mu \mathrm{l}$ of liposome containing buffer. To determine the protein content of the fractions, a Bradford assay was performed for each liposome 
Materials and Methods

population. The liposomes were frozen in liquid nitrogen and stored at $-80^{\circ} \mathrm{C}$. Occasionally, the integrity and size of the liposomes was checked for by electron microscopy. The proteoliposomes containing the neuronal SNAREs had a diameter between $30 \mathrm{~nm}$ to $50 \mathrm{~nm}$. The preparations were analyzed on 15\% SDS-PAGE.

\subsubsection{Digestion of proteoliposomes with toxins to determine protein orientation}

Tetanus toxin and Botulinum $\mathrm{C}$ are clostridial neurotoxins, the light chains of which are highly selective SNARE proteases. Whereas Tetanus toxin light chain selectively cleaves synaptobrevin between residues 76 and 77 (Eisel et al., 1986; Fairweather et al., 1986), Botulinum C cleaves syntaxin between residues 253 and 254 (Hauser et al., 1990). The toxins cleave only if the SNAREs are surface exposed in reconstituted liposomes. This was used as a tool to determine the orientation of synaptobrevin or syntaxin incorporated in liposomes. Synaptobrevin liposomes were incubated with Tetanus toxin light chain and syntaxin liposomes were incubated with BoNT $\mathrm{C}$ light chain at $37^{\circ} \mathrm{C}$ for $1-2 \mathrm{~h}$ in the presence or absence of $1 \%$ CHAPS. Reactions were stopped by SDS containing sample buffer. The samples were analyzed on $15 \%$ SDS-PAGE followed by Coomassie staining or were documented with an LAS-1000 reader (Fujifilm) using the appropriate filter sets (HQ470/40x (Cy2) Exiter / HQ525/50m (Cy2) Emitter, and HQ545/30x (Cy3) Exiter / HQ610/75m (Cy3) Emitter).

\subsubsection{Preparation of synaptic vesicles from rat brain}

Synaptic vesicles were purified from rat brains as described in detail in previous publications (Hell, 1994; Huttner et al., 1983; Nagy et al., 1976). All materials and reagents were kept detergent free to avoid disruption of the vesicles. The entire prep was carried out at $4^{\circ} \mathrm{C}$. 20 whole rat brains were homogenized in Hepes-buffered at $\mathrm{pH} 7.2$ $0.32 \mathrm{M}$ sucrose using a glass/teflon homogenizer (9 strokes at $900 \mathrm{rpm}$ ). PMSF and pepstatin A were added immediately (to a final concentration of $0.001 \% \mathrm{~V} / \mathrm{v}$ using a 200 $\mathrm{mM}$ stock solution). Centrifugation was carried out in Sorvall SS-34 rotor for $10 \mathrm{~min}$ at $800 \mathrm{x} \mathrm{g} \mathrm{(2700} \mathrm{rpm).} \mathrm{The} \mathrm{supernatant} \mathrm{(S1)} \mathrm{was} \mathrm{again} \mathrm{centrifuged} \mathrm{in} \mathrm{SS-34} \mathrm{rotor} \mathrm{for} 15 \mathrm{~min}$ at $9200 \mathrm{x} \mathrm{g}(10000 \mathrm{rpm})$. The pellet (P2) was resuspended in $120 \mathrm{ml} 0.32 \mathrm{M}$ sucrose and centrifuged in the SS-34 rotor for $15 \mathrm{~min}$ at $9200 \mathrm{x} \mathrm{g}(11000 \mathrm{rpm})$. The resuspended pellet (P2') contained the crude synaptosomal fraction and was resuspended in $24 \mathrm{ml} 0.32$ 
M Hepes-buffered sucrose. The crude synaptosomal fraction was subjected to osmotic lysis by adding nine volumes of distilled water to the resuspended pellet and homogenized in a glass/teflon homogenizer (3 strokes at $2000 \mathrm{rpm}$ ). $1.2 \mathrm{ml}$ of Hepes-NaOH pH 7.2 and PMSF were added. The lysate was centrifuged in the SS-34 rotor for $20 \mathrm{~min}$ at 25,000 $\mathrm{xg}$ $(16,500 \mathrm{rpm})$. The supernatant (LS1) was removed and spun in a Beckmann ultracentrifuge $\mathrm{Ti} 70$-rotor for $2 \mathrm{~h}$ at $165000 \mathrm{x} \mathrm{g}(50000 \mathrm{rpm})$. The pellet (LP2) was resuspended and homogenized in $6 \mathrm{ml}$ of $40 \mathrm{mM}$ sucrose, 3 strokes at $3000 \mathrm{rpm}$ in glass teflon homogenizer. Subsequently, the homogenate was taken in with a $20 \mathrm{G}$ needle and pressed through a $27 \mathrm{G}$ needle. This crude synaptic vesicle fraction was loaded on top of a continuous sucrose gradient (top: $50 \mathrm{mM}$ sucrose; bottom: $800 \mathrm{mM}$ sucrose) and centrifuged in a SW 28-rotor for $4 \mathrm{~h}$ at 65,000 x g (23000 rpm). The vesicle-containing band was pumped out of the gradient (approximately at 200-400 $\mathrm{mM}$ sucrose). This fraction was loaded on top of the controlled pore glass bead column and eluted over night in buffered glycine (5 mM Hepes-KOH, pH 7.3, $300 \mathrm{mM}$ glycine). The flow rate was adjusted to approximately $40 \mathrm{ml} / \mathrm{h}$. The absorbance at $280 \mathrm{~nm}$ wave-length was recorded and fraction sizes of 5-6 ml were collected. Vesicle containing fractions were pooled (the second peak of the UV-recording corresponds to the synaptic vesicles) and spun in an ultracentrifuge, Ti 50.2-rotor for $2 \mathrm{~h}$ at $165,000 \mathrm{x} \mathrm{g}(50000 \mathrm{rpm})$. The pellet was resuspended in $0.5 \mathrm{ml}$ Hepes buffered at $\mathrm{pH} 7.2 \mathrm{~K}$-Glut solution, taken in with a $20 \mathrm{G}$ needle and pressed through a $27 \mathrm{G}$ needle. Protein concentration of the vesicles was determined by Bradford assay as described above. Occasionally, the integrity and size of the vesicles was checked for by electron microscopy. $50 \mu 1$ aliquots of purified synaptic vesicles were shock frozen in liquid nitrogen and stored at $-80^{\circ} \mathrm{C}$.

\subsubsection{Preparing and running SDS-PAGE gels (Laemmli, 1970)}

For SDS-PAGE gels the following components were mixed for the stacking and the separation gels (15\%). Ready-to-use gas stabilized solutions of $30 \%$ acrylamide and $0.8 \%$ bisacrylamide were used to prepare the gels.

Composition of $15 \%$ SDS-PAGE gel:

\begin{tabular}{|l|l|l|}
\hline \multicolumn{1}{|c|}{ Component } & \multicolumn{1}{c|}{$\begin{array}{c}\text { Stacking gel (3.75\%) } \\
\text { (4 gels) }\end{array}$} & \multicolumn{1}{c|}{$\begin{array}{c}\text { Separation gel (15\%) } \\
\text { (2 gels) }\end{array}$} \\
\hline Acrylamide $30 \%$ & $1.25 \mathrm{ml}$ & $5.0 \mathrm{ml}$ \\
\hline Gel buffer & $2.5 \mathrm{ml}$ & $2.5 \mathrm{ml}$ \\
\hline $\mathrm{H}_{2} \mathrm{O}$ & $6.14 \mathrm{ml}$ & $2.4 \mathrm{ml}$ \\
\hline TEMED & $10 \mu \mathrm{l}$ & $5 \mu \mathrm{l}$ \\
\hline $10 \%$ APS & $100 \mu \mathrm{l}$ & $100 \mu \mathrm{l}$ \\
\hline
\end{tabular}


The glass walls, spacers $(0.75 \mathrm{~mm})$ and the combs were cleaned with ethanol and mounted in the holder. $3.4 \mathrm{ml}$ of the separation gel were poured between the glass walls. This was overlayed with iso-butanol saturated with water to smoothen the interface between the stacking gel and the separation gel. After the polymerization of the separation gel, the iso-butanol was removed and the remaining volume was filled with stacking gel and the comb was inserted. After the gel had polymerized, the protein samples were mixed with the SDS loading buffer, either $2 \mathrm{x}$ or $5 \mathrm{x}$ sample buffer, and loaded either directly or boiled for $5 \mathrm{~min}$ before loading on the gel. The gel chamber was filled with running buffer. The proteins were loaded into the pockets of the gel and electrocuted at constant current of $15 \mathrm{~mA}$. After the blue front reached the separation gel boarder the current level was elevated to $25 \mathrm{~mA}$. The gel was allowed to run till the blue front diffused out of the gel. (Upper gel buffer: 0.5 M Tris(HCl, pH 6.8, 0.4\% SDS, Lower gel buffer: $1.5 \mathrm{M}$ Tris/HCl, $\mathrm{pH} 8.8,0.4 \% \mathrm{SDS})$.

\subsubsection{Coomassie-blue-staining}

After electrophoresis, the stacking gel was discarded and the separation gel was stained for $15-30 \mathrm{~min}$ in $50 \%(\mathrm{~V} / \mathrm{v})$ methanol, $10 \%(\mathrm{~V} / \mathrm{v})$ acetic acid and $0.2 \%(\mathrm{~W} / \mathrm{v})$ Coomassie Brilliant Blue R-250 while shaking. The gel was destained in 50\% (V/v) ethanol and 10\% (V/v) acetic acid for $15 \mathrm{~min}$ and then in $10 \%(\mathrm{~V} / \mathrm{v})$ ethanol and $5 \%(\mathrm{~V} / \mathrm{v})$ acetic acid until no background was left. The gels were scanned, dried in the gel dryer (Geldryer Model 538, Biorad) wrapped in cellophane foil.

\subsubsection{Immunoblotting}

Gels were blotted in a semi-dry transfer mode (Towbin et al., 1989) using nitrocellulose membranes (GB003 from Schleicher \& Schuell). Per membrane a current of $50 \mathrm{~mA}$ was applied for 50 minutes. The membrane was subsequently blocked by incubation in 5\% low fat milk solution for at least 30 minutes followed by incubation for at least $60 \mathrm{~min}$ at RT with the primary antibody at a 1:1000 or 1:2000 dilution in 5\% low fat milk solution. After three washing steps in wash buffer $(0.2 \mathrm{M}$ TrisHCl $\mathrm{pH} 7.8,0.1 \%$ Tween-20 (V/v), 0.15M NaCl,) for at least 5 minutes each, horseradish peroxidase (HRP) or alkaline phosphatase (AP) conjugated secondary antibody was added (dilution 1:1000 to $1: 2000$ ) in 5\% low fat milk solution and incubated for 30 minutes. Again, three 
washing steps were performed before enhanced chemiluminiscence reagent (for HRPconjugated secondary antibody) was applied according to manufacturer's instruction (Perkin Elmer Western Lightning) and detected using the luminescent image analyzer (LAS-1000 Fujifilm). For AP- conjugated secondary antibody, a combination of nitro blue tetrazolium $(0.33 \mathrm{mg} / \mathrm{ml})$ and 5-bromo-4-chloro-3-indolyl phosphate $(0.17 \mathrm{mg} / \mathrm{ml})$ was applied and detected by eye.

\subsubsection{Immunoprecipitation}

Immunoprecipitation was done essentially as described in (Edelmann et al., 1995). Lysate pellet 2 (LP2, pre-incubated or not with soluble syntaxin and SNAP-25 for $1.5 \mathrm{~h}$ ) or P2' (crude synaptosomal fraction) was dissolved in ice-cold extraction buffer (20 mM Hepes-KOH pH 7.3, 140 mM KCl, 2 mM EDTA,1-1.5\% Triton X-100 or 1\% CHAPS). Protein amount was adjusted to 1 to $1.5 \mathrm{mg} / \mathrm{ml}$, and the insoluble material was removed by centrifugation for $30 \mathrm{~min}$ at $100,000 \mathrm{x}$ g. $7.5 \mu \mathrm{l}$ of ascites fluid corresponding to $\sim 8-25$ $\mu \mathrm{g}$ specific IgG per $\mathrm{ml}$ solution were added and incubation was carried out for $8-10 \mathrm{~h}$ at $4^{\circ} \mathrm{C} .75 \mu \mathrm{l}$ of protein G- Sepharose suspension (GE Healthcare) $/ \mathrm{ml}$ of detergent extract was incubated for 1-1.5 h. The beads were collected by centrifugation and washed three times in extraction buffer before elution with sample buffer containing SDS and Bmercaptoethanol.

\subsubsection{Cross-linking}

Cross-linking was done as described in (Edelmann et al., 1995). For cross-linking, LP2 was resuspended at a protein concentration of 1 to $1.5 \mathrm{mg} / \mathrm{ml}$ in Krebs-Ringer buffer (1.2 mM Na2HPO4, $5 \mathrm{mM} \mathrm{NaHCO3,} 140 \mathrm{mM} \mathrm{NaCl}, 1 \mathrm{mM} \mathrm{MgCl}$,) and pre-warmed for 10 min at room temperature. Disuccinimidyl suberate (DSS) was dissolved in DMSO at a final concentration of $5 \mathrm{mM}$. Crosslinking was carried out at RT for $45 \mathrm{~min}$. The reaction was quenched with Tris-Cl, $\mathrm{pH} 7.4,(100 \mathrm{mM}$ final concentraion) for $30 \mathrm{~min}$ at RT. The membranes were pelleted in a microfuge at $5000 \mathrm{rpm}$ for $3 \mathrm{~min}$ and then resuspended in 1 $\mathrm{ml}$ ice-cold buffer (20 mM Tris-Cl pH 7.4, $150 \mathrm{mM} \mathrm{NaCl}$ ) containing 1\% Triton X-100 and incubated at $4^{\circ} \mathrm{C}$ for $1 \mathrm{~h}$. Insoluble material was removed by centrifugation at 40000 $\mathrm{rpm}(100,000 \mathrm{~g})$ for $20 \mathrm{~min}$ in TLA 55 rotor. $20 \mu \mathrm{l}$ aliquots of the supernatants were analyzed by SDS-PAGE followed by immunoblotting. 


\subsubsection{In vitro formation of SNARE complexes}

Binary and ternary complexes used in the Coomassie staining and immunoblotting studies were formed overnight (unless otherwise stated) in the stoichiometric ratios stated in the figure legends. Complex formation was verified by nondenaturing PAGE (binary complexes) or SDS-PAGE (ternary complexes). Some of the complexes were purified by anion-exchange chromatography (MonoQ, GE Healthcare).

\subsubsection{Tetanus toxin protection assay}

Tetanus toxin cleaves synaptobrevin 2 specifically between glutamine 76 and phenylalanine 77 . When synaptobrevin is in the complex, tetanus toxin cannot cleave it. Protection of synaptobrevin from cleavage is an indication of the cleavage site being occluded in a complex. SNARE complex was generated as described above. Subsequently, the volume was filled up to $50 \mu 1$ with K-Glut buffer $(120 \mathrm{mM}$ potassium glutamate, $20 \mathrm{mM}$ potassium acetate, $20 \mathrm{mM}$ Hepes, $\mathrm{pH}$ 7.2) and $1 \mu 1$ of recombinantly expressed tetanus toxin light chain was added (corresponding to $1.2 \mu \mathrm{g}$ of protein and a final concentration of approximately $500 \mathrm{nM}$ ). Equal amount of proteoliposomes of the same preparation alone served as control. The solutions were incubated for $1 \mathrm{~h}$ at $37^{\circ} \mathrm{C}$ before SDS-PAGE.

\subsubsection{Absorption Spectroscopy}

The labeling efficiencies were determined from the absorption spectra, according to the Beer-Lambert law.

$$
\mathrm{A}_{\mathrm{\varepsilon}} \quad \frac{\mathrm{MW} \text { of protein }}{\mathrm{mg} \text { protein } / \mathrm{ml}}=\frac{\text { moles of dye }}{\text { moles of protein }}
$$

The extinction coefficients were used as given by the provider ( $\varepsilon$ (Oregon Green) $490 \mathrm{~nm}=70000 \mathrm{M}-1 \mathrm{~cm}-1,(\varepsilon($ Texas Red $) 590=80000 \mathrm{M}-1 \mathrm{~cm}-1))$. All measurements were carried out in a Shimadzu (UV-2401 PC) spectrophotometer. ( $A_{x}$ represents Absorption)

\subsubsection{Fluorescence Anisotropy}

All measurements were carried out in a Fluoromax-2 (Horiba Jobin Yvon) with automated polarizers (Model: 1971) or Fluorolog-3 (Horiba Jobin Yvon) with in-built 
polarizers essentially as described in (Fasshauer and Margittai, 2004). Excitation and emission wavelengths were $488 \mathrm{~nm}$ and $520 \mathrm{~nm}$, respectively (Oregon green). The slit widths were set between $2.5 \mathrm{~nm}$ and $3.5 \mathrm{~nm}$ to ensure fluorescence intensities between 80,000 and $100,000 \mathrm{cps}$. The integration time was set at $1 \mathrm{sec}$. The G-factor was determined according to $\mathrm{G}=\mathrm{I}_{\mathrm{HV}} / \mathrm{I}_{\mathrm{HH}}$, where $\mathrm{I}_{\mathrm{HV}}$ and $\mathrm{I}_{\mathrm{HH}}$ are the fluorescence intensities of the vertically and horizontally polarized emissions when the sample is excited with horizontally polarized light. After measuring the intensities of the vertically and horizontally polarized emissions upon vertically polarized excitation $\left(\mathrm{I}_{\mathrm{VV}}\right.$ and $\mathrm{I}_{\mathrm{VH}}$, respectively), the anisotropy values were computed automatically by the software according to following relationship: $\mathrm{r}=\left(\mathrm{I}_{\mathrm{VV}}-\mathrm{GI}_{\mathrm{VH}}\right) /\left(\mathrm{I}_{\mathrm{VV}}+2 \mathrm{GI}_{\mathrm{VH}}\right)$ (Lakowicz, 2000). The concentration of the labeled protein was always kept between $70 \mathrm{nM}$ and $300 \mathrm{nM}$. To start SNARE assembly, the unlabeled reactants were always added in large excess to saturate the reaction. The temperature was kept constant at $25^{\circ} \mathrm{C}$ throughout the experiment. All assembly reactions were carried out in quartz cuvettes (Hellma) in a final volume of 2.5 $\mathrm{ml}$.

\subsubsection{Förster Resonance Energy Transfer (FRET)}

Förster resonance energy tranfer (FRET) experiments were carried out in Fluoromax-2 spectrometer (Horiba Jobin Yvon) or Fluorolog-3 (Horiba Jobin Yvon) as described in (Fasshauer and Margittai, 2004). All measurements were carried out at $25^{\circ} \mathrm{C}$ ( $37^{\circ} \mathrm{C}$ for disassembly) in 1-cm quartz cuvettes (Hellma) in PBS (unless otherwise stated). FRET measurements were done by donor excitation at $488 \mathrm{~nm}$ and monitoring donor (OG) fluorescence emission $(520 \mathrm{~nm})$ and acceptor (TR) fluorescence emission $(610 \mathrm{~nm})$. The slit widths were set to $1-4 \mathrm{~nm}$ and the integration time was set to $1 \mathrm{~s}$. Spectra were measured before and after the reaction. The change in fluorescence intensity over time was observed for both the donor and the acceptor molecules. All assembly and disassembly reactions were carried out in quartz cuvettes (Hellma) in a final volume of $2.5 \mathrm{ml}$ and $2 \mathrm{ml}$ respectively.

FRET was also monitored in 96-well plates in a total volume of $100 \mu 1$ in GeniosPro (Tecan) using excitation filter of $490 \mathrm{~nm}$ and and emission filters of $510 \mathrm{~nm}$ and $610 \mathrm{~nm}$. The maximum gain was set to 40 and the signal at a time was set to 10 flashes. 


\subsubsection{Kinetic Simulations}

Simulations were performed on the complete 4D data matrix (time, wavelength, fluorescence, concentration) with the specialized 4D data fitting software Pro-Kineticist II (Applied Photophysics, UK), a second order global and local analysis software. For fitting, the residuals and the resulting sum of squares were calculated by Newton-Raphson algorithm and numerical integration. Subsequent iterations were performed using the derivative of the residuals matrix and shifts in the rate and formation constants. $K_{\text {on }}$ and $\mathrm{K}_{\text {off }}$ were determined in the global analysis mode for reaction intermediates and products by the simultaneous analysis of multiple datasets gathered at different sets of initial conditions.

\subsubsection{Antibody binding to ternary complex on synaptic vesicles}

Soluble syntaxin (4.7 nmoles) and SNAP-25 (3.43 nmoles) were incubated with purified synaptic vesicles $(117 \mu \mathrm{g})$ overnight. $20 \mu \mathrm{l}$ of ascites cleared by centrifugation at $100,000 \mathrm{x} \mathrm{g}$ for $15 \mathrm{~min}$ in TLA 120.2 rotor was incubated with synaptic vesicles +/syntaxin/SNAP-25. The mixes were pelleted at 100,000 x g for $15 \mathrm{~min}$ in TLA 120.2 rotor. After three washes in PBS, the pellets were resuspended in $50 \mu 1$ of the same buffer, $5 \mu 1$ of which was analyzed in SDS-PAGE followed by Coomassie staining.

\subsubsection{Extraction of rat brain cytosol}

Cytosol was prepared from the brains of freshly killed male adult Wistar rats (Klenchin et al., 1998). The brain stem and cerebellum were not used. Brains were placed into isotonic solution (50 mM PIPES, pH 6.8, $130 \mathrm{mM} \mathrm{NaCl}, 2 \mathrm{mM}$ EGTA, 1 protease inhibitor cocktail tablet, Boehringer Mannheim, Germany; $1 \mathrm{ml} / \mathrm{brain}$ ) and homogenised with 1 slow and 3 fast strokes of a Teflon homogeniser running at $900-1000 \mathrm{rpm}$ at $4^{\circ} \mathrm{C}$. Larger debris was removed by centrifugation at $14500 \mathrm{~g}$ in SS-34 rotor for 15 minutes at $4^{\circ} \mathrm{C}$. The supernatant was collected and centrifuged at $128000 \mathrm{~g}$ (rotor TLA 100.3, Beckman) for $60 \mathrm{~min}$ at $4^{\circ} \mathrm{C}$ in order to remove mitochondria and myelin. The second supernatant was dialysed against 41 of ice-cold K-Glut buffer for $2 \mathrm{~h}$, with a change of buffer every half an hour. Cytosol was aliquoted, frozen in liquid nitrogen and stored at $80^{\circ} \mathrm{C}$. Protein concentration was determined according to Bradford (Bradford, 1976). 


\subsubsection{1 [3H]-NE release assay from PC-12 cells}

Streptolysin-O based PC-12 cells release assay was adapted from Klenchin et al. (Klenchin et al., 1998). PC-12 cells were cultured in uncoated 6-well plates in [3H] NE $(7 \mu \mathrm{Ci} / 15 \mathrm{~cm}$ diameter plate, stock $40 \mathrm{Ci} / \mathrm{mmol}$, Amersham Biosciences) containing medium for 9-12 h. Medium was removed and fresh medium not containing the radiolabel was added to the culture for the 'chase' period. Cells were subsequently placed on ice and washed with PBS and PBS/0.2\% BSA followed by incubation with Streptolysin-O (75 $\mu \mathrm{g} / \mathrm{ml}$, final concentration) for $10 \mathrm{~min}$ on ice. After another wash with PBS to remove excess Streptolysin-O, cells were incubated for $12 \mathrm{~min}$ at $37^{\circ} \mathrm{C}$ in permeabilisation buffer (20 mM Hepes, pH7.2, $120 \mathrm{mM} \mathrm{KCl,} 20 \mathrm{mM} \mathrm{K}$-Acetate, $4 \mathrm{mM} \mathrm{MgCl}_{2}$ ) in the presence or absence of antibodies in $0.1 \%$ BSA. For stimulation, $\mathrm{Ca}^{+2}(10 \mu \mathrm{M}$ final conc.), Rat brain cytosol (2 mg/ml, final concentration), EGTA (5 mM, final concentration), ATP (2 mM, final concentration), Creatine phosphate (CP, $26 \mathrm{mM}$, final concentration) and Creatine Kinsae (CK, 0.132 mg (800 units/mg) Roche, Basel, Switzerland) were added to the permeabilisation buffer. For negative control, calcium, ATP, CP, CK and rat brain cytosol were omitted. Stimulation was done for $25 \mathrm{~min}$ at $37^{\circ} \mathrm{C}$ with $0.1 \%$ BSA. Supernatants were removed and pellets were solubilized in Triton X-100. Activity was measured in a liquid scintillation counter.

\subsubsection{Cell culture}

Experiments were performed using the neuroendocrine cell line PC-12 clone 251 (Heumann et al., 1983). PC-12 cells were cultured in $75 \mathrm{~cm}^{2}$ uncoated flasks in DMEM with $4.5 \mathrm{~g} / 1$ glucose supplemented with $10 \%$ fetal calf serum (FCS), 5\% horse serum, 4 $\mathrm{mM} \mathrm{L}$-glutamine and $60 \mathrm{U} / \mathrm{ml}$ each of penicillin and streptomycin at $37^{\circ} \mathrm{C}$ in $10 \% \mathrm{CO} 2$. For splitting or plating on coverslips, cells were washed with PBS and detached with $3 \mathrm{ml}$ trypsin-EDTA (Bio-Whittaker Europe, Verviers, Belgium) for 1 min. Trypsin activity was quenched by addition of $15 \mathrm{ml}$ growth medium and the cells were centrifuged at $235 \mathrm{~g}$ at $20^{\circ} \mathrm{C}$ for $5 \mathrm{~min}$. The pellet was titurated in growth medium. Aliquots of approximately $2 \mathrm{x}$ $10^{5}$ cells were seeded on $25 \mathrm{~mm}$ coverslips coated with poly-L-lysine. After additional 15 minutes to allow the cells to settle, $2.5 \mathrm{ml}$ of growth medium was added. Cells were used for the experiments at least $24 \mathrm{~h}$ after. 


\subsubsection{Preparation of active synaptosomes}

Synaptosomes were prepared from adult (250 grams) rats. Four rats were decapitated, the brains were removed into ice-cold sucrose buffer $(5 \mathrm{mM}$ Hepes, $\mathrm{pH} 7.4$, $320 \mathrm{mM}$ sucrose,). The cortex and cerebellum were homogenized in $90 \mathrm{ml}$ of sucrose buffer (10 strokes at $900 \mathrm{rpm}$, in presence of protease inhibitors, $0.2 \mathrm{mM}$ PMSF and 1 $\mu \mathrm{g} / \mathrm{ml}$ of pepstatin A). Cellular fragments were pelleted by centrifugation at $2000 \mathrm{~g}$ (2 minutes) in a Sorvall SS34 rotor. The supernatant was centrifuged further at $9500 \mathrm{~g}$ for 12 minutes, rendering a pellet consisting mainly of synaptosomes, mitochondria and myelin. The pellets were resuspended (avoiding the relatively harder mitochondrial component) in $10 \mathrm{ml}$ sodium buffer (20 mM Hepes pH 7.4, $140 \mathrm{mM} \mathrm{NaCl}, 5 \mathrm{mM} \mathrm{KCl,} 10 \mathrm{mM}$ glucose, $5 \mathrm{mM} \mathrm{NaHCO}_{3}, 1.2 \mathrm{mM} \mathrm{Na}_{2} \mathrm{HPO}_{4}, 1 \mathrm{mM} \mathrm{MgCl}_{2}$ ) and centrifuged at $9500 \mathrm{~g}$. They were then resuspended in 1-2 $\mathrm{ml}$ sodium buffer and used within 1 hour for release experiments.

\subsubsection{Synaptosome stimulation}

Synaptosomes were pre-incubated at $37^{\circ} \mathrm{C}$ for 20 minutes. $\mathrm{Ca}^{2+}(1.3 \mathrm{mM})$ or $5 \mathrm{mM}$ EGTA were added for a further 10 minutes. Stimulation was then performed for 5-7 minutes by addition of $50 \mathrm{mM} \mathrm{KCl}$ or $10 \mu \mathrm{M}$ ionomycin, followed by placing the synaptosome-containing tubes on ice and immediate disruption by hypo-osmotic shock (addition of a 9-fold higher volume of ice-cold water, and homogenization using a glass teflon homogenizer, six strokes at maximum speed). The plasma membranes and smaller vesicles were pelleted separately by differential centrifugation (see section on synaptic vesicles preparation).

\subsubsection{Immunocytochemistry}

Immunocytochemistry was done essentially as described in (Willig et al., 2006). PC-12 cell cultures were fixed for 30-45 minutes with 4\% paraformaldehyde, quenched with $50 \mathrm{mM}$ ammonium chloride (in PBS) for 15 minutes, permeabilized with $0.1 \%$ Triton X 100, and incubated for 1 hour with primary antibodies diluted at 1:100 in PBS/1\% BSA $/ 0.1 \%$ Triton X 100. The coverslips were then washed three times with PBS/0.1\% Triton X-100, and secondary antibodies were added for one hour. The cultures were then washed with high salt PBS (three times, $500 \mathrm{mM} \mathrm{NaCl}$ in $20 \mathrm{mM}$ sodium phosphate buffer, $\mathrm{pH} 7.4)$, low salt $\mathrm{PBS}$ (150 mM NaCl in $10 \mathrm{mM}$ sodium phosphate 
buffer, pH 7.4) and PBS, mounted in Dako mounting medium (DakoCytomation) and imaged.

\subsubsection{Fluorescence microscopy}

A Zeiss Axiovert 200M fluorescence microscope was used, equipped with a 1.4 NA 100x objective and a CCD camera with a 1317 x 1035 Kodak chip (pixel size 6.8 x $6.8 \mu \mathrm{m}$, Princeton Instruments Inc., USA). Cy3 fluorescence (red channel) was detected using the excitation filter 565/30X-HQ, the beamsplitter 595 DCLP-Q, and the emission filter 645/45M-HQ. Cy2 fluorescence was detected with the excitation filter 480/40X-HQ, the beamsplitter 505 DCLP-HQ, and the emission filter 527/30M-HQ.

\subsubsection{Direct labeling of synaptic vesicles}

Highly enriched synaptosomes were obtained by Ficoll gradient centrifugation (Nicholls and Sihra, 1986), and synaptic vesicles were generated (LS1). They were then labeled and processed as published previously (Brandhorst et al., 2006). Imaging and data analysis was performed as previously described (Brandhorst et al., 2006). Experiments were performed by S.O. Rizzoli (MPI-bpc, Göttingen).

\subsubsection{Mass-spectrometry}

Twenty bands were cut out from a lane of 15\% SDS-PAGE in which the immunoprecipitate had been run. Proteins were digested with trypsin and peptides were extracted from the gel. Extracted peptides were subjected to nano LC-coupled Electrospray Ionization (ESI) tandem mass spectrometry. Proteins were identified by searching the fragment pattern of the sequenced peptides against the NCBInr database using Mascot as search engine. Experiments were performed by Henning Urlaub (MPIbpc, Göttingen). 


\subsubsection{Generation, immuonostaining, image acquisition and data analysis of membrane sheets}

Experiments with plasma membrane sheets were done as described in (Lang et al., 2002). Membrane sheets were generated from cells grown on poly-L-lysine-coated coverslips by using a 100-ms ultrasound pulse in ice-cold K-Glut buffer (20 mM Hepes, pH 7.2, 120 mM K-Glutamate, 20 mM K-Acetate, 10 mM EGTA). The membrane sheets were either directly fixed in $4 \%$ paraformaldehyde (PFA) or incubated in a humid

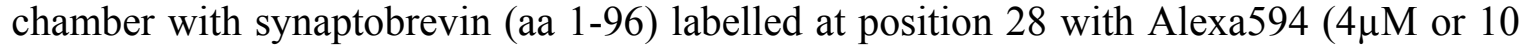
$\mu \mathrm{M})$ at $37^{\circ} \mathrm{C}$ in $\mathrm{K}-$ Glut buffer containing 3\%BSA for 30-60 min. Membrane sheets were then washed with PBS (10 mM NaH$\left.{ }_{2} \mathrm{PO}_{4}, \mathrm{pH} 7.4,10 \mathrm{mM} \mathrm{Na}_{2} \mathrm{HPO}_{4}, 150 \mathrm{mM} \mathrm{NaCl}\right)$ two times for 10 min each at RT followed by fixing for $30 \mathrm{~min}$ at RT with $4 \%$ PFA dissolved in PBS. PFA was quenched for $20 \mathrm{~min}$ in PBS containing $50 \mathrm{mM} \mathrm{NH}{ }_{4} \mathrm{Cl}$. Incubation with primary antibody (1-2 h, 1:100 dilution ) and following washing steps (2x $10 \mathrm{~min}$. each) were carried out in PBS (+/- $1 \%$ BSA and $+/-500 \mathrm{mM} \mathrm{NaCl}$ as indicated). Incubation with secondary antibody (1-2 h, goat-anti-rabbit-Cy2, 1:200 dilution) was carried out in PBS. Membrane sheets were then washed three times in PBS for $10 \mathrm{~min}$, and imaged in 4\% 1-(4-trimethylammonium)-6-phenyl-1,3,5-hexatriene (TMA-DPH)saturated PBS solution. In some experiments fluorescent beads (tetraspeck beads) were used as a spatial reference.

Fluorescence microscopy and quantification of immunofluorescence intensity was performed essentially as previously described (Lang et al., 2002). For correlation analysis, regions of a size of several $\mu \mathrm{m}^{2}$ were extracted from both channels using the program Metamorph (Universal Imaging corporation, West Chester, PA, USA) and imported in the program Sigma Plot (Systat Software, Inc., Richmond, USA). The Pearson Corrleation coefficient was calculated according to $\mathrm{r}=\Sigma_{\mathrm{i}}\left(\right.$ green $_{\mathrm{i}}-$ green $\left._{\mathrm{av}}\right) *\left(\operatorname{red}_{\mathrm{i}}-\operatorname{red}_{\mathrm{av}}\right) /\left\{\sum_{\mathrm{i}}\left(\operatorname{green}_{\mathrm{i}^{-}}\right.\right.$ green $\left.\left._{\mathrm{av}}\right)^{2} * \sum_{\mathrm{i}}\left(\mathrm{red}_{\mathrm{i}}-\mathrm{red}_{\mathrm{av}}\right)^{2}\right\}^{1 / 2}$ (Manders et al., 1992). For each experiment comparing 135.1 staining with syb 2-Alexa594 labeling, 5-14 individual membrane sheets (on average 10) were analysed, and three experiments were performed. The mean \pm S.E.M. are given. One experiment was performed comparing 69.1 staining with syb 2-Alexa594 labeling, analysing 15 individual membrane sheets. 


\section{$3 \quad$ Results}

\subsection{SNARE complex assembles on artificial vesicles}

Studies probing the reactivity and the assembly pathway of neuronal SNAREs, the putative fusogens, are essential to the understanding of factors that govern neuroexocytosis. Cytoplasmic domains of neuronal SNAREs are known to spontaneously assemble into a tight ternary complex. This complex is resistant to SDS treatment and migrates in SDS-PAGE without dissociating into its monomers, thus indicating SNARE complex formation (Hayashi et al., 1994). SNARE complexes dissociate into their individual components upon heating (Fig. 4). Extensive studies have enhanced understanding of the assembly pathway of soluble domains of SNAREs, reviewed in (Fasshauer, 2003). It was proposed that the soluble domain of synaptobrevin readily binds to the syntaxin/SNAP-25 acceptor complex (Fasshauer and Margittai, 2004). The reactivity of synaptobrevin reconstituted in liposomes, however, has been called into question. It was proposed that synaptobrevin in membranes is refractory to SNARE complex formation, thus requiring additional factors for activation $\mathrm{Hu}$ et al., 2002; Kweon et al., 2003b). These studies imply that SNAREs are incapable of interacting with each other in their native environment, unless an activating factor, probably $\mathrm{a} \mathrm{Ca}^{+2}$ sensor, relieves the inhibition of synaptobrevin. A major goal of this work, therefore, was to examine the reactivity of membrane-anchored synaptobrevin.

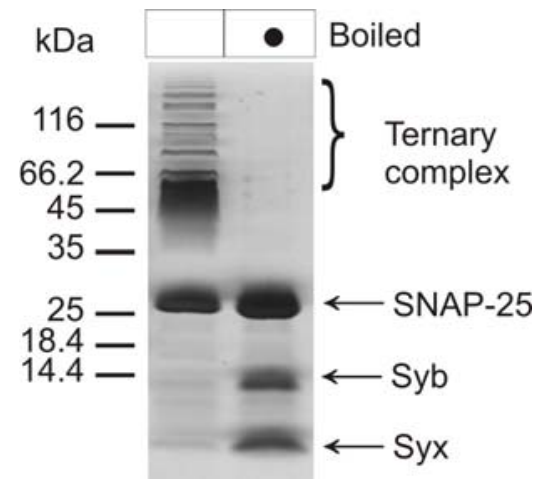

Figure 4. SNARE complex formation using soluble domains of SNAREs, monitored by SDSPAGE and Coomassie Blue staining.

Incubation of syntaxin 1, SNAP-25 and synaptobrevin (aa 1-96, Syb) in the stoichiometric ratio of 2 (SNAP-25): 1 (syntaxin): 1 (synaptobrevin) results in the appearance of SDS-resistant bands of higher molecular mass (Ternary complex). The SDS-resistant bands dissociate into their monomeric constituents when the sample is boiled prior to SDS-PAGE. 


\subsubsection{Liposomes do not influence assembly of soluble domains of SNAREs}

The proposed inhibition of synaptobrevin could be traced to its interaction with the membrane. This inhibition may arise from the interaction of its interfacial residues (aa 77-90) with membrane, as this domain has been shown to bind to membranes (Quetglas et al., 2000). Alternatively, the trans-membrane region could confer structural features rendering it incapable of contacting its binding partners. To test the former possibility, i.e., whether the assembly of soluble domains of SNAREs is influenced by lipids, SNARE complex formation was monitored by Förster resonance energy transfer (FRET) in the presence or absence of protein free liposomes. Versions of synaptobrevin (aa 1-96) and SNAP-25 (full-length) in which single cysteines were introduced at positions 28 and 130 respectively were purified and labelled with Oregon Green $(\mathrm{OG})$ as donor $\left(\mathrm{Syb}^{28 \mathrm{OG}}\right)$ and Texas Red (TR) as acceptor fluorophore (SNAP-25 ${ }^{130 T R}$ ), respectively. The rate of SNARE complex assembly, reported by donor fluorescence quenching, does not change significantly when approximately $100 \mu \mathrm{M}$ protein-free liposomes was included in the reaction (Fig. 5), suggesting that the assembly of SNAREs without intact trans-membrane regions is independent of their interaction with the lipids.

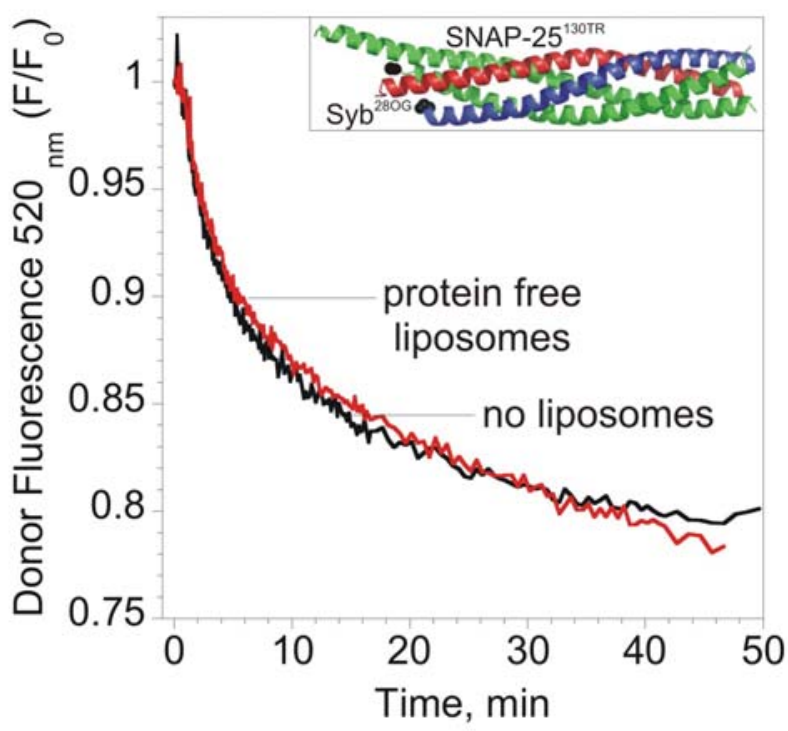

Figure 5. SNARE complex formation of soluble synaptobrevin (aa 1-96), syntaxin (aa 180-262) and SNAP-25 in the presence or absence of protein free liposomes.

Complex formation was monitored by FRET (donor quenching). Synaptobrevin, labeled at position 28 with Oregon Green ( $\mathrm{Syb}^{28 \mathrm{OG}}$, final conc $\sim 200 \mathrm{nM}$.) was incubated with SNAP-25 labeled at position 130 with Texas Red (SNAP-25 ${ }^{130 \mathrm{TR}}$, final conc. $\sim 300 \mathrm{nM}$ ). Upon addition of syntaxin $(\sim 500 \mathrm{nM})$, donor fluorescence decreased, indicating complex 
formation. Note that the presence of protein free liposomes did not alter the rate of complex formation of soluble SNAREs. Insert: cartoon of SNARE complex crystal structure indicating the labeling positions (black spheres).

\subsubsection{SNARE complex assembles on syntaxin proteoliposomes}

In the next set of experiments I asked whether syntaxin inserted in liposomes is active and readily engages in SNARE complexes with its cognate SNAREs. To test whether membrane-bound syntaxin is sufficient to interact with synaptobrevin and SNAP25 , the trans-membrane domain containing syntaxin with either only the SNARE motif (aa 180-288) or full-length (aa 1-288) were reconstituted into liposomes. Liposomes were generated from cholate extracts using size-exclusion chromatography and further purified on Nycodenz density gradients. Syntaxin-containing liposomes were incubated with soluble synaptobrevin and SNAP-25 for $6 \mathrm{~h}$ at room temperature (RT). As shown in Fig. 6 , both versions of syntaxin in liposomes engage soluble synaptobrevin and SNAP-25 to form SNARE complexes that are detectable as SDS-resistant bands of higher molecular weight.

a
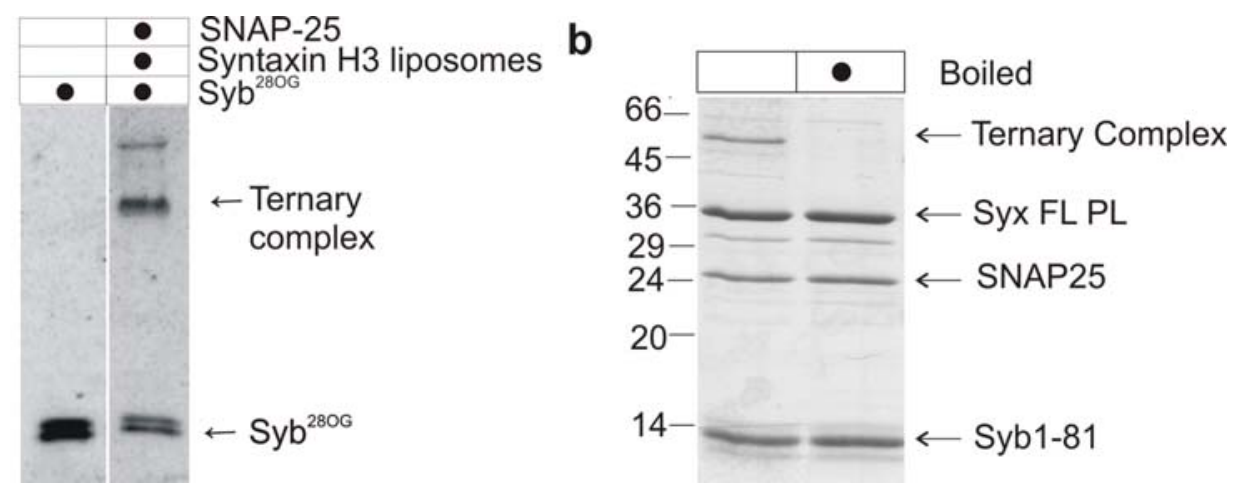

Figure 6. SNARE complex formation on syntaxin (Syx-) liposomes monitored by SDS-PAGE followed fluorescence detection or Coomassie staining.

a) SNARE complex formation on syntaxin (aa 180-288) liposomes monitored by SDSPAGE followed by detection of fluorescence derived from synaptobrevin. Incubation of syntaxin liposomes (120 pmoles) with SNAP-25 (500 pmoles) and soluble synaptobrevin (aa 1-96) labeled at position 28 with Oregon green ( $\mathrm{Syb}^{280 \mathrm{G}}, 250$ pmoles) results in the appearance of SDS-resistant bands of higher molecular mass (Ternary complex). The reaction was carried out in a total volume of $50 \mu \mathrm{l}$ for $6 \mathrm{~h}$ and was stopped by adding $50 \mu \mathrm{l}$ 2x sample buffer containing SDS; $5 \mu l$ of this reaction was analyzed.

b) SNARE complex formation on full length syntaxin (aa 1-288) liposomes monitored by SDS-PAGE and Coomassie staining. Syntaxin full-length liposomes (Syx FL PL, 30 pmoles) was incubated with SNAP-25 (50 pmoles) and soluble synaptobrevin (aa 1-81, 50 pmoles). A band of higher molecular mass (Ternary complex) was visible which dissociated into monomeric SNAREs when boiled. 


\subsubsection{SNARE complex assembles on synaptobrevin proteoliposomes}

The results described above indicate that syntaxin inserted in liposomes can engage soluble synaptobrevin and SNAP-25 in SNARE complexes. Does synaptobrevin reconstituted in liposomes form SNARE complex with the soluble domain of syntaxin and SNAP-25? Several reports suggest that membrane-inserted synaptobrevin is largely inactive (Hu et al., 2002; $\mathrm{Hu}$ et al., 2003; Kweon et al., 2003b): when isolated synaptic vesicles were incubated with recombinant SNAP-25 and syntaxin 1, no complex formation was observed (Kweon et al., 2003b). Furthermore, when recombinant synaptobrevin incorporated in liposomes was incubated with syntaxin and SNAP-25, complexes were not detected unless membrane-solubilizing detergent was added suggesting that synaptobrevin is intrinsically inactive when inserted into a bilayer (Kweon et al., 2003b). To test these findings and further resolve the issue of SNARE complex assembly on synaptobrevin liposomes, purified synaptobrevin was incorporated into liposomes in a manner similar to what has been described for syntaxin above. Reconstitution results in random orientation with nearly $50 \%$ of the protein pointing out of the lumen of the liposomes, as seen by the cleavage of synaptobrevin by the light chain of Tetanus toxin (TeNT) (Fig. 7). When the liposomes were treated with TeNT in the presence of membrane solubilizing detergent, the entire pool of synaptobrevin was cleaved by the toxin. 


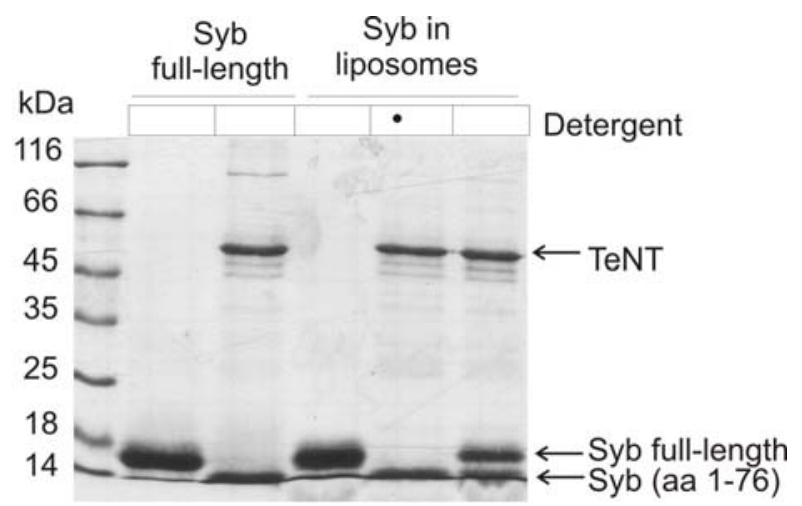

Figure 7. Orientation of synaptobrevin reconstituted in liposomes.

Synaptobrevin proteoliposomes were incubated with Tetanus toxin light chain (TeNT) in the absence or presence of membrane-solubilizing detergent (2\% CHAPS) and analyzed on an SDSPAGE followed by Coomassie Blue staining. Full-length synaptobrevin was completely cleaved by TeNT resulting in a fragment of synaptobrevin (aa 1-76) indicating that the protease was active. Almost $50 \%$ of synaptobrevin inserted in liposomes was cleaved by TeNT suggesting random orientation. When synaptobrevin proteoliposomes were treated with TeNT in the presence of detergent, the cleavage was complete.

To investigate whether the correctly oriented synaptobrevin in liposomes is capable of forming SNARE complexes, recombinant SNAP-25 (full-length) and syntaxin were incubated with synaptobrevin liposomes in a stoichimetric ratio of 2 (synaptobrevin): 1 (SNAP-25): 1 (syntaxin). As shown in Fig. 8a, SNARE complexes formed that are detectable as SDS-resistant bands. The remaining synaptobrevin was resistant to toxin cleavage indicating that the entire pool of surface exposed synaptobrevin was recruited into SNARE complexes. Heating dissociated the complexes into their monomers (Fig. 8), as is usual for SNARE complexes.

a

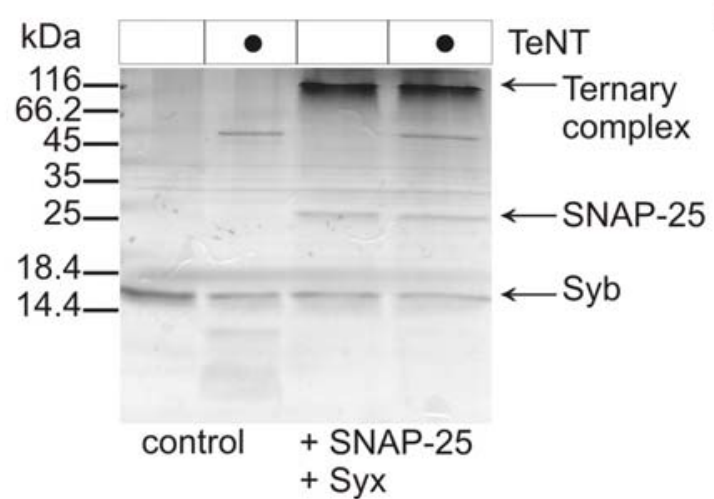

b

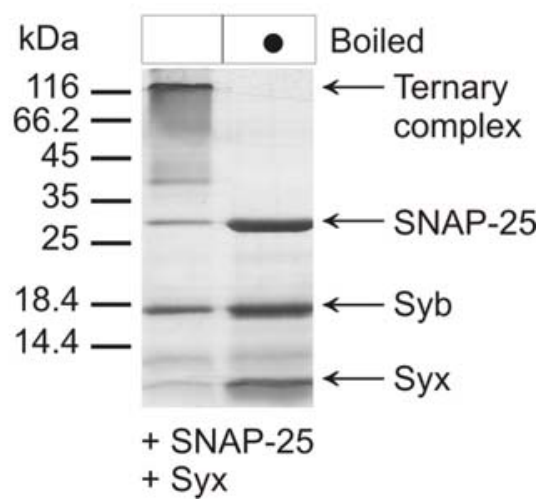

Figure 8. SNARE complex formation on synaptobrevin (Syb)-containing liposomes, monitored by SDS-PAGE and Coomassie Blue staining.

a) Addition of syntaxin 1 (aa 180-262, Syx) and SNAP-25 results in the appearance of SDSresistant bands of higher molecular mass (Ternary complex). Treatment of liposomes with the light chain of tetanus toxin (TeNT) before addition of SNAP-25 and syntaxin reduced 
synaptobrevin by about 50\% (left lanes). After SNARE complex formation, the remaining synaptobrevin was resistant to toxin cleavage (right lanes), suggesting that this pool is oriented towards the vesicle interior. About 300 pmoles of synaptobrevin in liposomes was incubated with 150 pmoles of SNAP-25 and of syntaxin overnight.

b) The SDS-resistant bands dissociate into their monomeric constituents when the sample is boiled prior to SDS-PAGE. Assembly was carried out as in a).

\subsubsection{Fluorescence spectroscopy assays report kinetics of SNARE complex formation on artificial vesicles}

As a measure of kinetics of SNARE complex assembly, complex formation was monitored by fluorescence anisotropy using single cysteine mutants of synaptobrevin labelled with the fluorophore Oregon Green $\left(\mathrm{Syb}^{280 \mathrm{G}}, \mathrm{Syb}^{79 \mathrm{OG}}, \mathrm{Syb}^{61 \mathrm{OG}}\right.$ ) incorporated in liposomes. To validate that the fluorescent labels on synaptobrevin did not sterically hinder SNARE complex formation, it was incubated with recombinant SNAP-25 and syntaxin in the same stoichiometric ratio as described for Fig. 8. As shown in Fig. 9a, SDS-resistant bands of higher molecular weight representing the ternary complex formed, documenting that the fluorophores do not prevent complex formation. Anisotropy represents the rotational motion of a fluorophore during its excited-state lifetime, thereby reporting local conformational flexibility of the dye that increases upon increasing constraints on the fluorophore. An increase of anisotropy, denoting complex formation, was observed for all labelling positions of synaptobrevin in liposomes upon addition of unlabelled syntaxin and SNAP-25 (Fig. 9), which was prevented in the presence of unlabelled soluble syaptobrevin. 


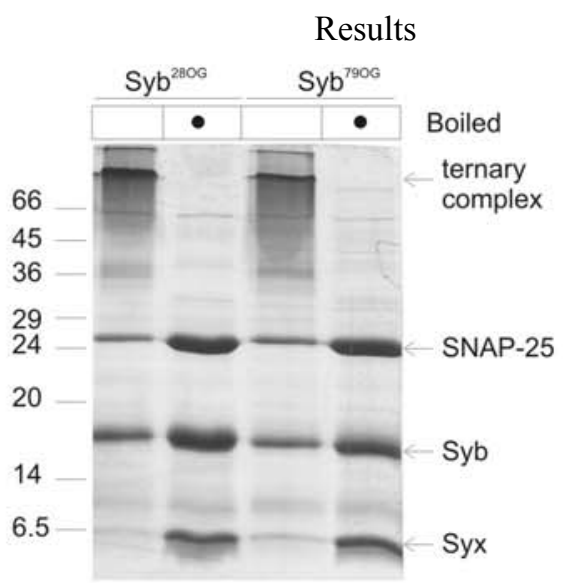

b

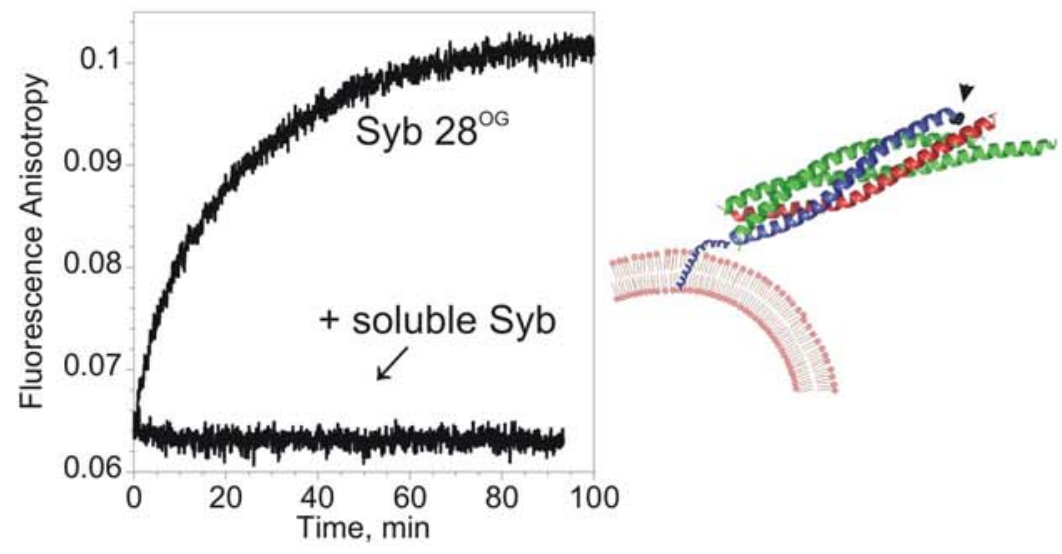

c
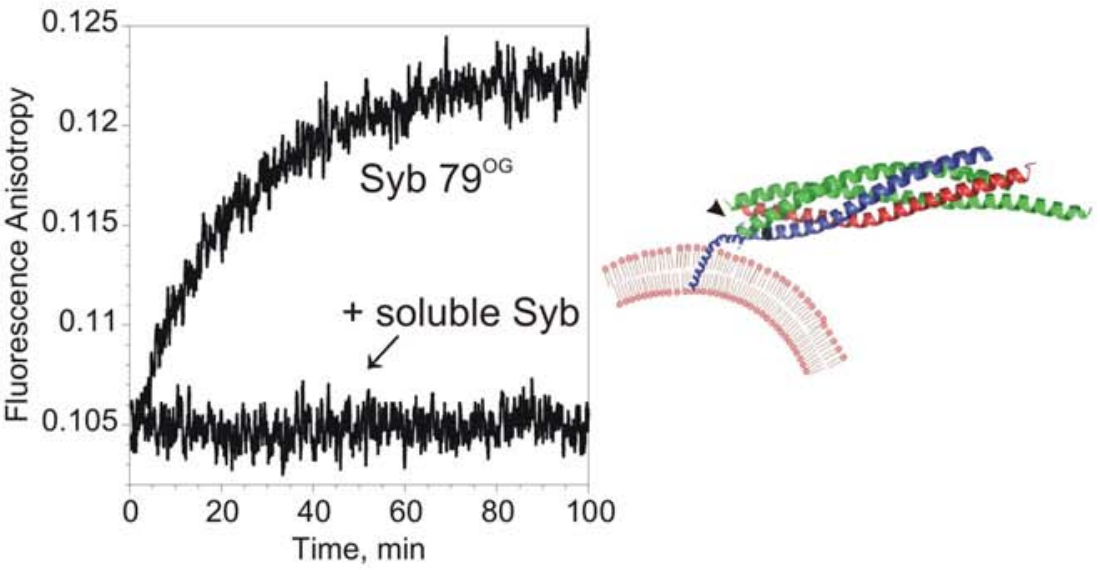

d
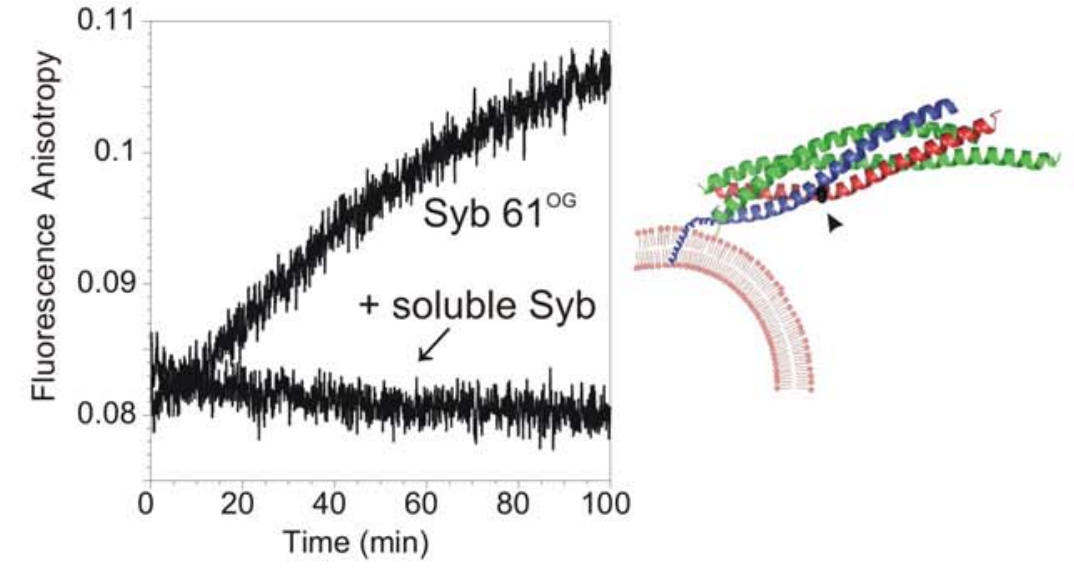
Figure 9. SNARE complex formation on synaptobrevin (Syb)-containing liposomes monitored by SDS-PAGE and fluorescence anisotropy.

a) SNARE complex formation with fluorescently labeled synaptobrevin monitored by SDSPAGE and Coomassie Blue staining to show that the fluorescent label does not hinder its capacity of engaging syntaxin and SNAP-25 in a complex. Synaptobrevin labeled at positions 28 or 79 with Oregon Green ( $\mathrm{Syb}^{280 \mathrm{G}}$ and $\mathrm{Syb}^{790 \mathrm{G}}$ respectively) incorporated in liposomes were incubated with syntaxin and SNAP-25 essentially as described in Fig. 8. SDS-resistant bands of higher molecular mass (ternary complex) appeared which dissociated into their monomers upon boling.

b) c) and d) Complex formation monitored by fluorescence anisotropy, using $\mathrm{Syb}^{610 \mathrm{G}}$ (b), $\mathrm{Syb}^{790 \mathrm{O}}$ (c) and $\mathrm{Syb}^{280 \mathrm{G}}$ (d) containing liposomes (all $\sim 100 \mathrm{nM}$ ). Anisotropy increased when unlabeled soluble syntaxin $(1.25 \mu \mathrm{M})$ and SNAP-25 $(1.92 \mu \mathrm{M})$ were added to the reaction. Excess soluble synaptobrevin $(5 \mu \mathrm{M})$ blocks the increase in anisotropy. Cartoons indicate the labeling position (black spheres) on synaptobrevin in liposome in the crystal structure of the SNARE complex.

To characterize SNARE complex assembly on membranes in more detail, FRET was used to report complex formation. Single cysteine mutants of each of the SNAREs was purified and labelled with either Oregon Green $(\mathrm{OG})$ as donor $\left(\mathrm{Syb}^{28 \mathrm{OG}}, \mathrm{Syb}^{790 \mathrm{O}}\right.$, $\mathrm{Syb}^{61 \mathrm{OG}}$ ) or Texas Red (TR) as acceptor (SNAP-25 ${ }^{130 \mathrm{TR}}$, SNAP-25 ${ }^{84 \mathrm{TR}}$, Syx ${ }^{225 \mathrm{TR}}$, Syx $\left.{ }^{197 T R}\right)$. FRET was monitored between the fluorophores on synaptobrevin and SNAP25 or between the fluorophores on synaptobrevin and syntaxin. In the crystal structure of the SNARE complex, all FRET pair positions are adjacent to each other on the outside of the complex, thus allowing to monitor complex formation while not interfering with the binding sites. As shown in Fig. 10a, FRET between $\mathrm{Syb}^{280 \mathrm{G}}$ containing liposomes and SNAP- $25^{130 T R}$ results in altered emission spectra upon donor excitation maxima at 488 $\mathrm{nm}$ before and after SNARE complex formation. Upon incubation with detergent (CHAPS, final conc.1\%) for $1 \mathrm{~h}$, further signal change was observed, in agreement with the finding that a fraction of synaptobrevin is oriented into the lumen of liposomes. FRET between $\mathrm{Syb}^{790 \mathrm{OG}}$ containing liposomes and SNAP-25 $5^{84} \mathrm{TR}$ was observed when unlabelled syntaxin was added to the reaction. Donor fluorescence decreased (upper panel, Fig. 10b) while acceptor fluorescence increased (lower panel, Fig. 10b), indicating complex formation. 
a
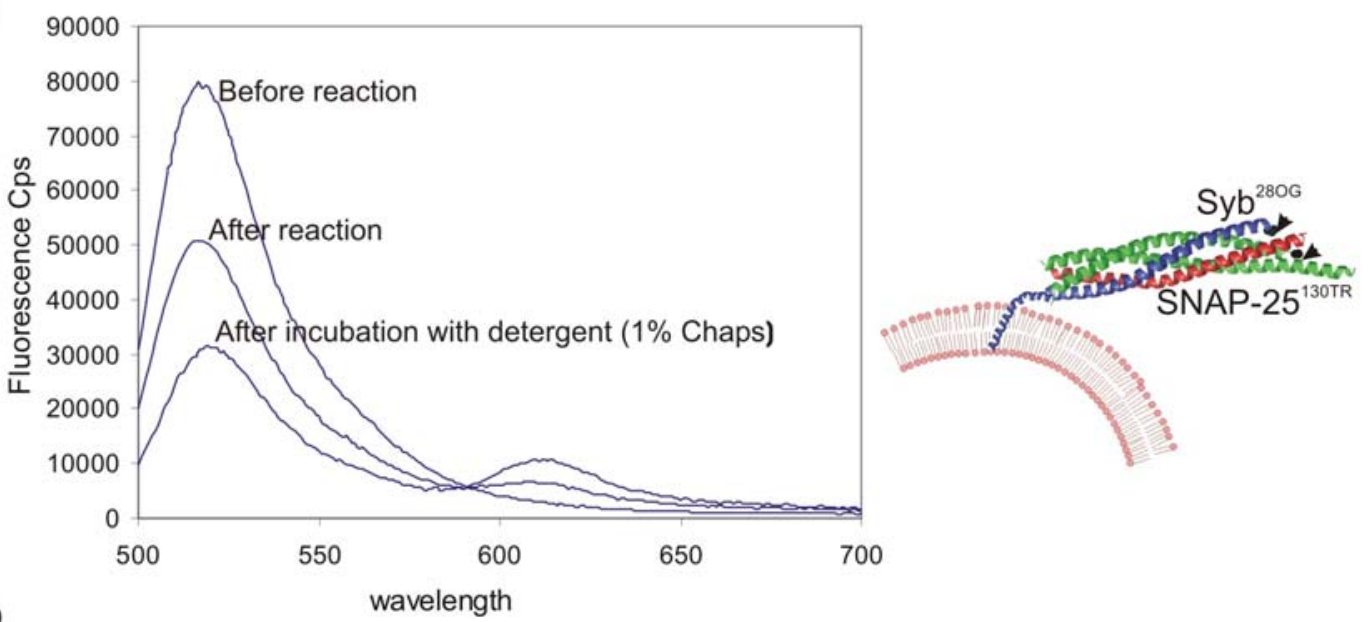

b
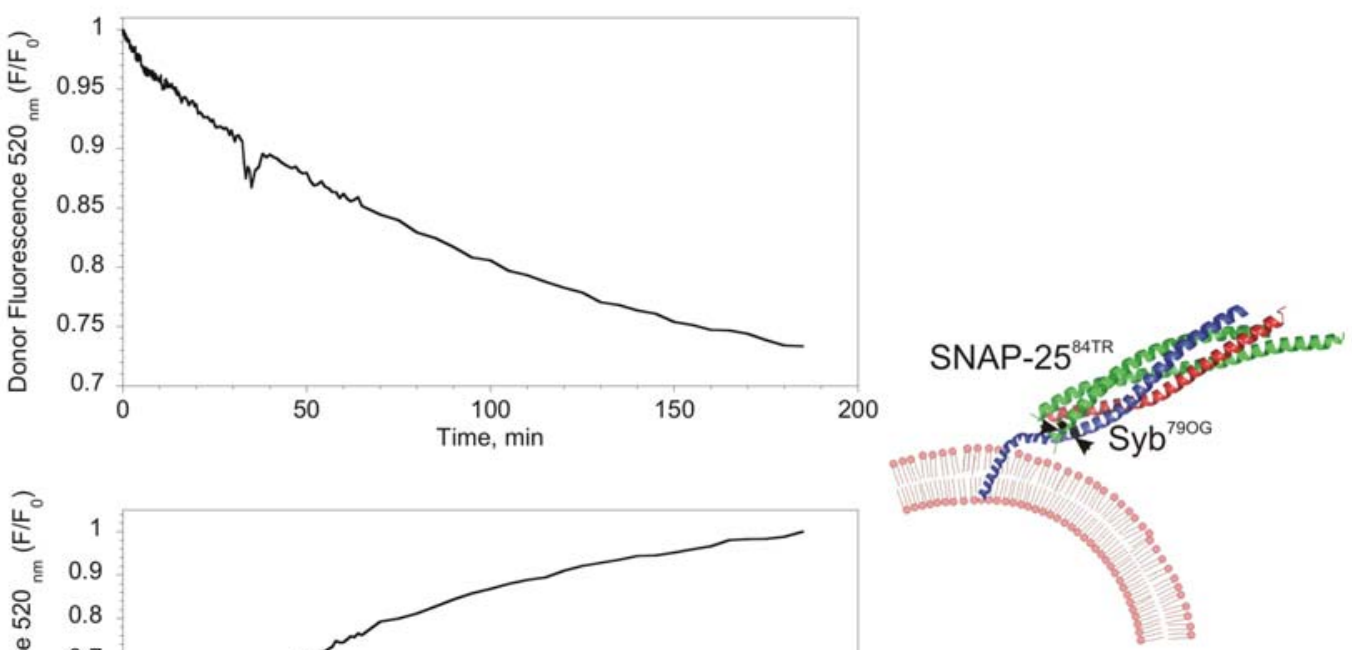

Figure 10. SNARE complex formation on synaptobrevin (Syb)-containing liposomes, monitored by FRET between synaptobrevin and SNAP-25

a) FRET between $\mathrm{Syb}^{280 \mathrm{O}}$ containing liposomes and SNAP-25 ${ }^{130 \mathrm{TR}}$. Emission spectrum $\left(\lambda_{\text {ex }}\right.$ $488 \mathrm{~nm}$, donor $\lambda_{\mathrm{em}} 520 \mathrm{~nm}$ and acceptor $\left.\lambda_{\mathrm{em}} 610 \mathrm{~nm}\right)$ was recorded before and after incubation of $\mathrm{Syb}^{280 \mathrm{OG}}$ reconstituted in liposomes $(125 \mathrm{nM})$, SNAP-25 ${ }^{130 \mathrm{TR}}(\sim 300 \mathrm{nM})$ and syntaxin $(1.25 \mu \mathrm{M})$ for $\sim 1 \mathrm{~h}$. Upon incubation with detergent (CHAPS, final conc. $1 \%)$ for 1h, further increase in FRET was observed indicating that a fraction of synaptobrevin was oriented in the lumen of liposomes.

b) FRET between $\mathrm{Syb}^{790 \mathrm{OG}}$ containing liposomes and SNAP-25 labeled at postion 84 with Texas Red (SNAP-25 $5^{84}$ TR $)$ Syb SyOG $^{79}$ liposomes $(\sim 600 \mathrm{nM})$ was incubated with unlabeled syntaxin $(1.25 \mu \mathrm{M})$. Upon addition of SNAP-25 ${ }^{84}$ TR $(\sim 1.1 \mu \mathrm{M})$, donor fluorescence decreased (upper panel) while acceptor fluorescence increased (lower panel), indicating complex formation. Cartoons indicate labeling positions on synaptobrevin in liposomes and SNAP-25. 
SNARE complex assembly on membranes was further validated by FRET pairs between synaptobrevin and syntaxin. FRET between $\mathrm{Syb}^{61 \mathrm{OG}}$ and Syx ${ }^{225 T R}$ (Fig. 11a) and $\mathrm{Syb}^{280 G}$ and Syx ${ }^{197 \mathrm{TR}}$ (Fig 11b) was observed upon addition of SNAP-25. Again, soluble unlabeled synaptobrevin effectively competed with the labeled synaptobrevin for complex formation. Furthermore, FRET was abolished when synaptobrevin liposomes were preincubated with trypsin (Fig. 11c), further documenting that the signal change observed was due to a specific interaction between the FRET pairs.

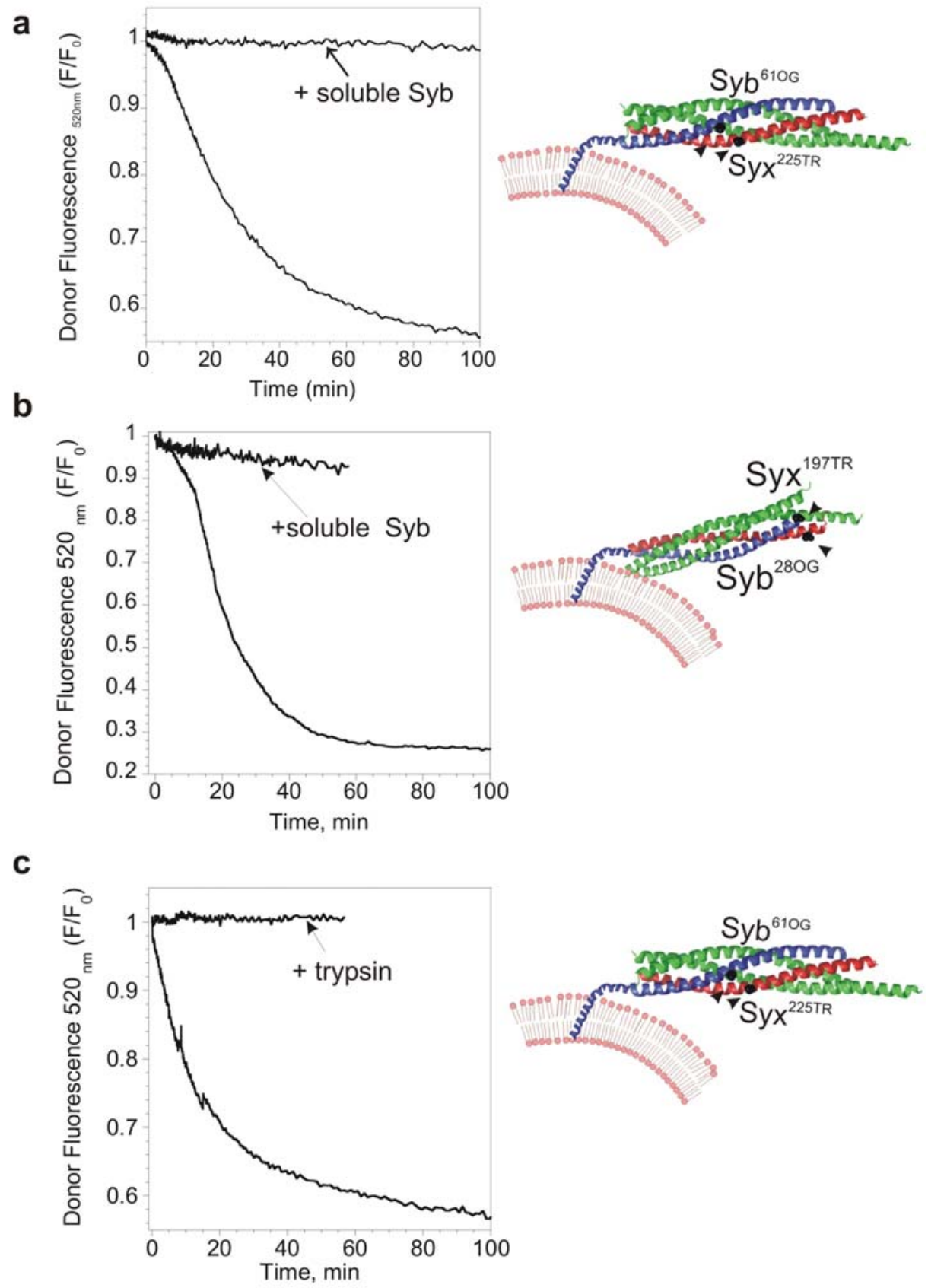


Figure 11. SNARE complex formation on synaptobrevin (Syb)-containing liposomes, monitored by FRET between synaptobrevin and syntaxin

a) and c) FRET between $\mathrm{Syb}^{610 \mathrm{G}}$ and syntaxin labeled at position 225 with Texas Red $\left(\mathrm{Syx}^{225 \mathrm{TR}}\right) . \mathrm{Syb}^{61 \mathrm{OG}}$, reconstituted in liposomes $(\sim 100 \mathrm{nM}$ final conc.) were incubated with $\operatorname{Syx}^{225 \mathrm{TR}}$ (final conc. $\sim 300 \mathrm{nM}$ ). Upon addition of SNAP-25 (1.28 $\mu \mathrm{M}$ (a), $2 \mu \mathrm{M}$ (c)) donor fluorescence decreased, indicating complex formation. Addition of soluble unlabeled synaptobrevin (a) $(2.5 \mu \mathrm{M}$ final conc.) effectively competed with the labeled synaptobrevin for complex formation. FRET was not observed when synaptobrevin liposomes was preincubated with $1 \mathrm{mM}$ trypsin for $2 \mathrm{~h}$ and quenched with PMSF prior to the reaction $(\mathrm{c})$

b) FRET between $\mathrm{Syb}^{280 \mathrm{G}}$ and syntaxin labeled at position 197 with Texas Red $\left(\mathrm{Syx}^{197 \mathrm{TR}}\right)$. $\mathrm{Syb}^{28 \mathrm{OG}}$, reconstituted in liposomes $\left(\sim 125 \mathrm{nM}\right.$ final conc.) was incubated with Syx ${ }^{197 \mathrm{TR}}$ (final conc. $\sim 370 \mathrm{nM}$ ). Upon addition of SNAP-25 (1.28 $\mu \mathrm{M}$ final conc.) donor fluorescence decreased, indicating complex formation. Addition of soluble unlabeled synaptobrevin $(\sim 2.5 \mu \mathrm{M}$ final conc.) effectively competed with the labeled synaptobrevin for complex formation.

Cartoons indicate labeling positions (black spheres) on synaptobrevin in liposomes and syntaxin.

\subsubsection{Reconstituted synaptobrevin engages SNAP-25 and syntaxin with the intact $\mathrm{N}$-terminal domain in a complex}

The results described above show that synaptobrevin in liposomes is fully available for SNARE complex assembly with SNAP-25 and the SNARE motif of syntaxin. The N-terminal region of syntaxin, known as the $\mathrm{H}_{\mathrm{abc}}$ domain, however, has been reported to have an inhibitory effect on SNARE complex formation (Margittai et al., 2003c). To determine whether synaptobrevin in liposomes is capable of engaging SNAP25 and syntaxin with the intact $\mathrm{N}$-terminal domain (syntaxin aa 1-262) in a complex, equimolar amount of all three proteins were mixed. As shown in Fig. 12a, SDS-resistant ternary complex was visible, which dissociated into its components upon heating. SNARE complex formation was also monitored by FRET. Synaptobrevin, labeled at position 61 with Texas Red was reconstituted in liposomes and incubated with syntaxin (aa 1-262) labeled at position 225 with Oregon Green. Upon addition of SNAP-25, FRET occurred, indicating complex formation (Fig. 12b). It is notable that the rate of complex formation with syntaxin (aa 1-262) is considerably slower than that with only the SNARE motif, as has been reported previously (Margittai et al., 2003c). 
a





Figure 12. SNARE complex formation on synaptobrevin (Syb)-containing liposomes with SNAP-25 and syntaxin (aa 1-262), i.e., syntaxin with the N-terminal Habc domain intact.

a) SNARE complex formation was monitored by SDS-PAGE and Coomassie Blue staining. Addition of entire cytoplasmic domain of syntaxin 1 (aa 1-262) and SNAP-25 results in the appearance of SDS-resistant bands of higher molecular mass (Ternary complex). Incubation with SNAP-25 and syntaxin (aa 1-262) was carried out overnight (all proteins being 50 pmoles). The SDS-resistant bands dissociate into their monomeric constituents when the sample is boiled prior to SDS-PAGE.

b) SNARE complex formation was monitored by FRET (donor quenching). Synaptobrevin, labeled at position 61 with Texas Red was reconstituted in liposomes $(\sim 150 \mathrm{nM}$ final conc.) and incubated with syntaxin (aa 1-262) labeled at position 225 with Oregon Green (final conc. $\sim 100 \mathrm{nM})$. Upon addition of SNAP-25 $(\sim 1.92 \mu \mathrm{M})$, donor fluorescence decreased indicating complex formation. For comparison, ternary complex formation using Syx 180-262 (see legend to Fig. 11) is shown (gray line).

\subsubsection{Synaptobrevin in aged or frequently frozen/thawed liposomes becomes refractory to SNARE complex assembly}

The results described so far demostrate clearly that SNARE complex assembles on synaptobrevin liposomes. It was noted, however, that synaptobrevin in liposomes becomes refractory to SNARE complex assembly after prolonged storage ( $>10$ days on ice) or frequent freeze-thaw cycles ( $>3$ times). As shown in Fig. 13a, complex formation on freshly prepared liposomes resulted in a shift of synaptobrevin to an SDS-resistant band of higher molecular weight whereas on aged liposomes, synaptobrevin did not enter SNARE complex. Treatment of freshly prepared liposomes with the light chain of tetanus toxin (TeNT) before addition of SNAP-25 and syntaxin cleaved nearly half of synaptobrevin corresponding to its random orientation in liposomes whereas synaptobrevin in aged liposomes was resistant to toxin cleavage (Fig. 13a). Using the FRET assay described above, (FRET between $\mathrm{Syb}^{61 \mathrm{OG}}$ and $\mathrm{Syx}^{225 \mathrm{TR}}$ ), donor fluorescence 
quenching was greatly retarded for synaptobrevin in aged liposomes. Results from electron microscopic analysis of liposomes (not shown) suggest that liposomes aggregate after prolonged storage. Together, these results indicate that though synaptobrevin in liposomes is fully available for complex formation, aged and frequently frozen and thawed liposomes tend to cluster and aggregate, thus rendering the synaptobrevin incorporated in them unavailable for complex formation.
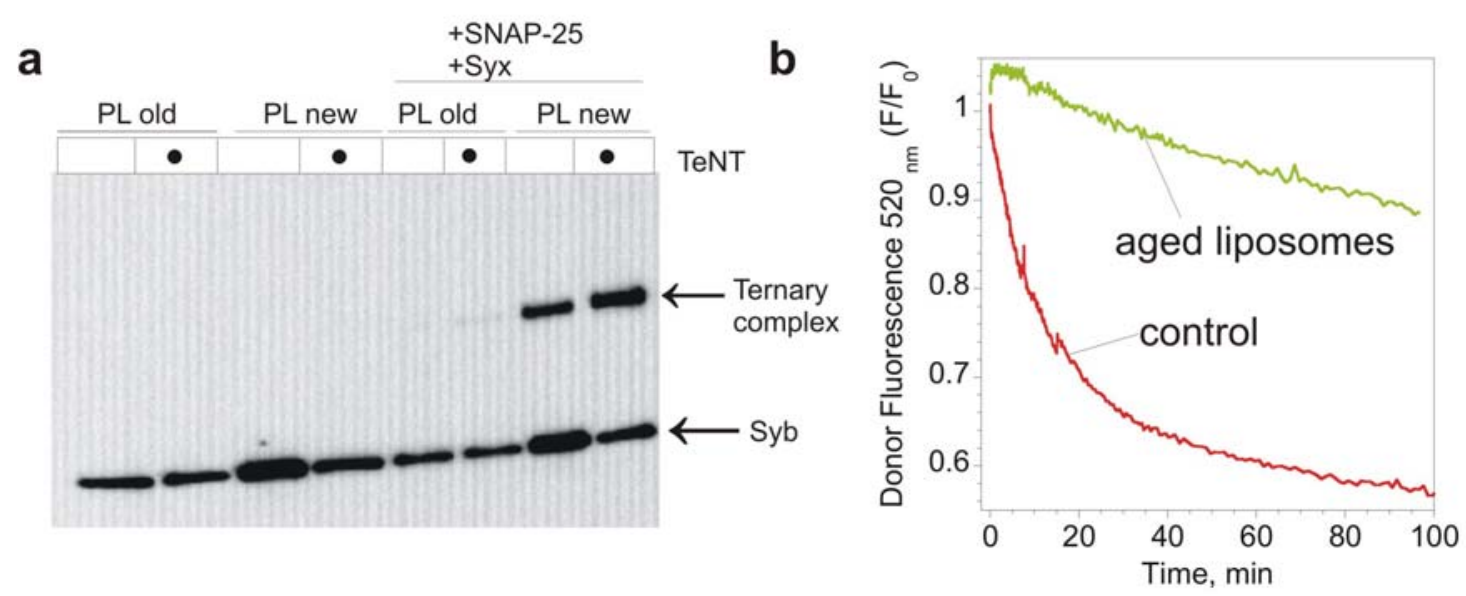

Figure 13. Synaptobrevin in liposomes becomes refractory to SNARE complex assembly after prolonged storage or frequent freeze-thaw cycles.

a) SNARE complex formation on synaptobrevin liposomes (Antonin et al.), monitored by SDSPAGE and immunoblotting for synaptobrevin. Syntaxin (40 pmoles) and SNAP-25 (100 pmoles) were incubated with synaptobrevin liposome (old and fresh, 80 pmoles) for $4 \mathrm{~h}$ in 50 $\mu \mathrm{l}$ volume. The reactions were stopped by adding $50 \mu 12 \mathrm{x}$ sample buffer containing SDS; $5 \mu 1$ of the reactions were analyzed.

b) SNARE complex assembly monitored by FRET ( $\left.\mathrm{Syb}^{61 \mathrm{OG}} / \mathrm{Syx}^{225 \mathrm{TR}}\right)$ as described in Fig. 11a.

\subsection{Kinetics of SNARE complex assembly on artificial vesicles}

The FRET assays described above enable the monitoring of the assembly of SNAREs on membranes. Likewise, the effects of extraneous and endogenous factors on SNARE complex assembly rate can be monitored. These studies could lead to a clearer understanding of the factors and conditions determining SNARE complex assembly on membranes. In view of the reports that reconstituted synaptobrevin is in an"off-state", unless membrane-solubilizng detergent is included (Hu et al., 2002; Kweon et al., 2003b), it is notable that in the present study, the rate of complex assembly increased only moderately when detergent (1\% CHAPS) was added to the reaction (Fig. 14), indicating 
that synaptobrevin in liposomes is not significantly retarded from forming SNARE complexes.

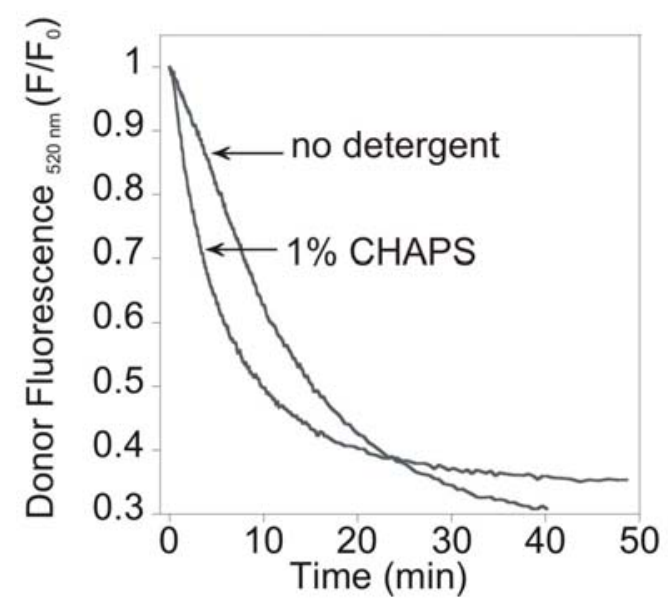

Figure 14. SNARE complex formation on synaptobrevin (Syb)-containing liposomes in the presence of detergent proceeds only moderately faster.

SNARE complex formation was monitored by FRET ( $\mathrm{Syb}^{61 \mathrm{OG}} / \mathrm{Syx}^{225 T R}$ ) (see legend to Fig. 11a) except that the the reaction carried out in the presence of detergent was in $1 \%$ CHAPS. Concentration of SNAP-25 was $\sim 1.92 \mu \mathrm{M}$.

\subsubsection{Assembly of SNARE complex on membranes proceeds via the syntaxin/ SNAP-25 binary complex}

It was shown earlier that a 1:1 complex between syntaxin and SNAP-25 is required for binding of synaptobrevin and that syntaxin competes with synaptobrevin for this binding, resulting in the formation of a non-productive 2:1 complex between syntaxin and SNAP-25 (Fasshauer et al., 2002; Fasshauer and Margittai, 2004). Excess of SNAP25 shifts the equilibrium to the 1:1 acceptor complex, which is expected to result in an enhanced rate of ternary complex formation. In my study, the FRET assay was used to investigate whether the rate of complex formation is dependent on the concentration of SNAP-25. Two different FRET pairs (Syb ${ }^{610 G}$ and Syx ${ }^{225 T R}$ ) (Fig. 15a) and (Syb ${ }^{280 G}$ and Syx ${ }^{197 T R}$ ) (Fig 15c) reported an increase in the rate of complex formation with increasing concentration of SNAP-25. No complex formation was observed in the absence of SNAP25 (Fig. 15a). Preincubation of syntaxin and SNAP-25 leads to a higher concentration of syntaxin/SNAP-25 acceptor complex, offsetting any time-delay for its formation. Addition of synaptobrevin to the preincubated mix would thus lead to a higher rate of 
complex formation than if all components were added sequentially, thereby precluding any delayed formation of the acceptor complex. As shown in Figs. 15b and 15d, this was indeed the case: preincubated reactions were significantly faster than when all components were added sequentially. 
a



Results

b

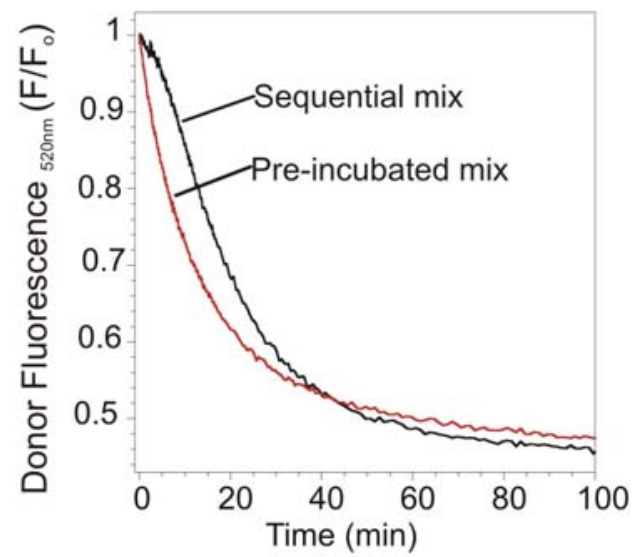

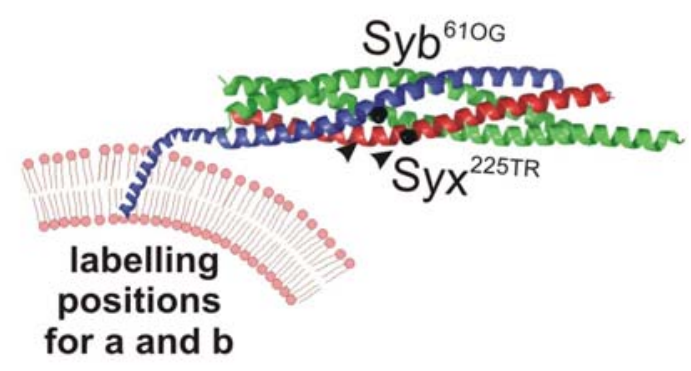

C

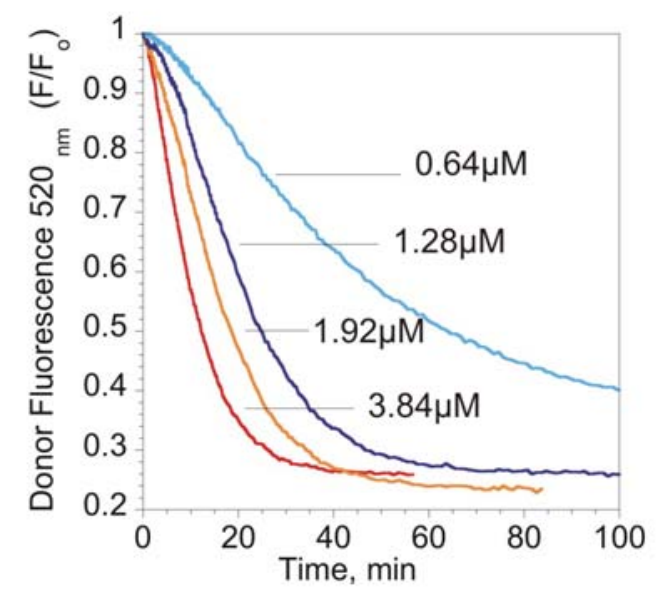

d

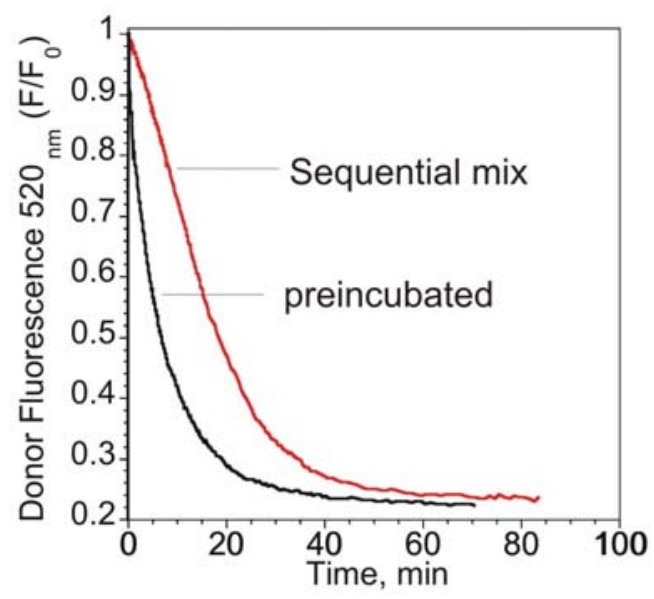

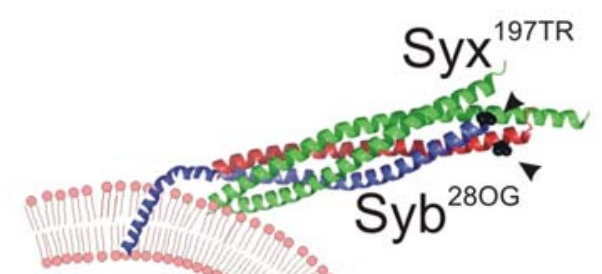

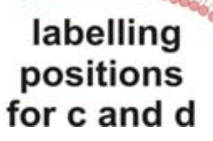

Figure 15. Increasing concentrations of SNAP-25 profoundly accelerates the rate of complex formation.

Complex formation was monitored by FRET (see legend to Fig. 11). 
a) and c) Increasing amounts of SNAP-25 shifted the equilibrium towards the 1:1 syntaxin/SNAP-25 acceptor complex, yielding higher concentration of binding sites for synaptobrevin thereby resulting in faster rate of SNARE complex formation

b) and d) Pre-mixing of syntaxin and SNAP-25 (FRET pair Syb ${ }^{610 G} / \mathrm{Syx}^{225 T R}$ final conc. $250 \mathrm{nM}$ and $1.28 \mu \mathrm{M}$ respectively and FRET pair $\mathrm{Syb}^{280 \mathrm{O}} / \mathrm{Syx}^{197 \mathrm{TR}}$ final conc. $125 \mathrm{nM}$ and $1.92 \mu \mathrm{M}$ respectively) results in a faster onset of complex formation indicating that the formation of acceptor complexes between syntaxin and SNAP-25 is initially ratelimiting.

\subsubsection{Kinetic Modelling of SNARE complex assembly on membranes}

The SNAP-25 titration experiments enables one to model the reaction pathway and determine $\mathrm{K}_{\mathrm{on}}$ and $\mathrm{K}_{\text {off }}$ of reaction intermediates. The SNAP-25 titration experiments were used to simulate the reaction scheme described in Fig. 16b. Applying the NewtonRaphson algorithm and numerical integration in an iterative process for minimization of residuals (Peduzzi et al., 1979), the simulated traces were fitted to the experimental ones (Fig. 16a, 17a and 18a). The goodness of the fit can be judged from the residuals that varied randomly around zero (Fig. $16 \mathrm{c}$ and d, $17 \mathrm{c}$ and $\mathrm{d}$ and $18 \mathrm{c}$ and $\mathrm{d}$ ). 
a
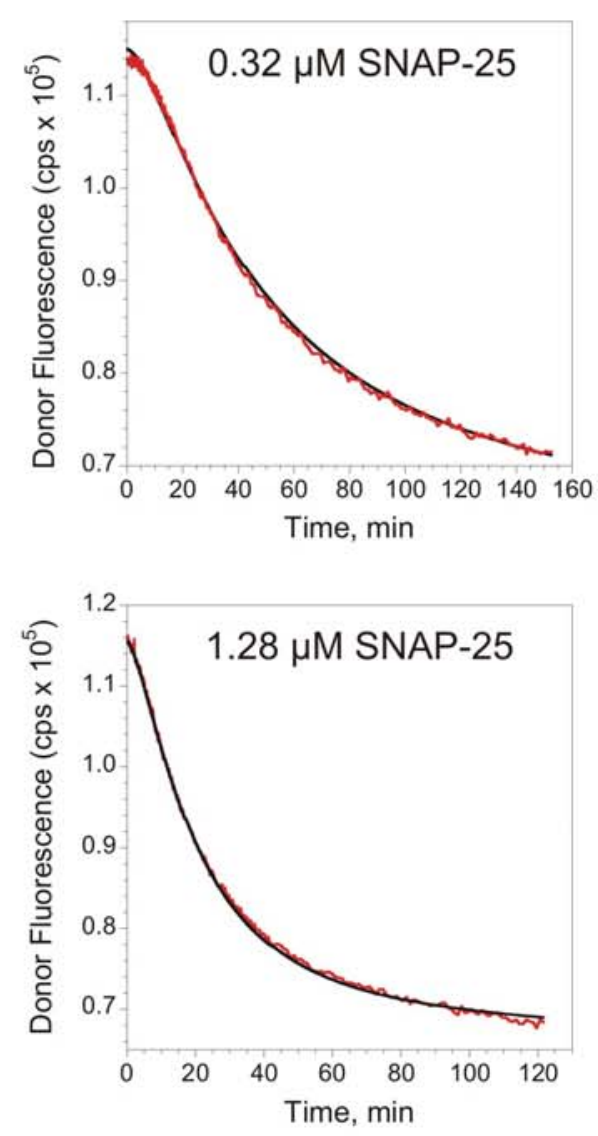
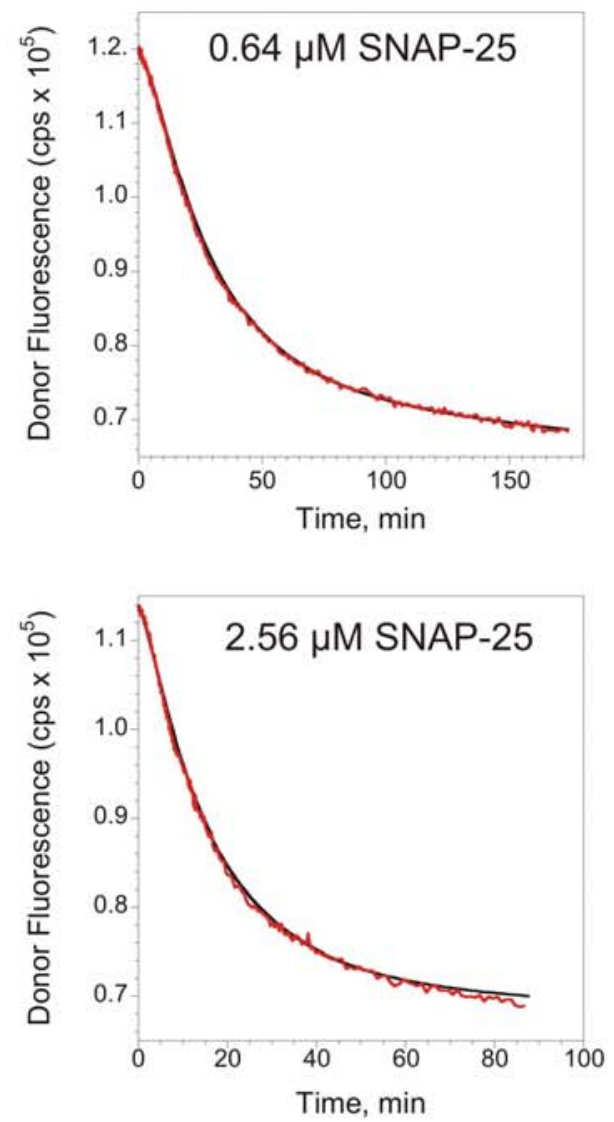

b

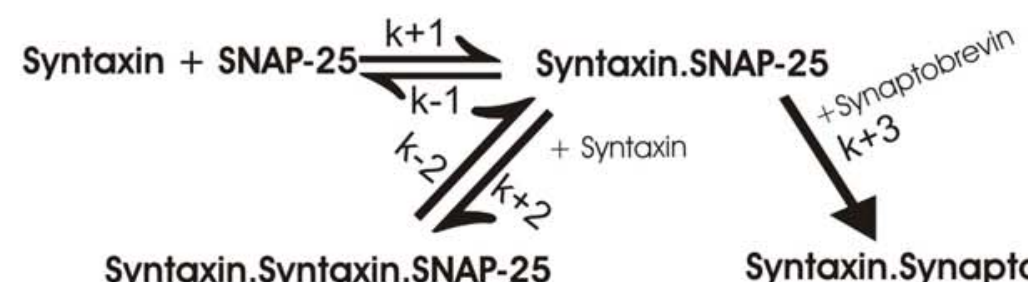

Syntaxin.Syntaxin.SNAP-25

Syntaxin.Synaptobrevin.SNAP-25

$\begin{array}{ll}\text { Name } & \text { Value } \\ \text { k+1: } & 1900 \mathrm{M}^{-1} \mathrm{~s}^{-1} \\ \mathrm{k}-1: & 0.0007 \mathrm{~s}^{-1} \\ \mathrm{k}+2: & 250 \mathrm{M}^{-1} \mathrm{~s}^{-1} \\ \mathrm{k}-2: & 1.060418 \mathrm{e}-010 \mathrm{~s}^{-1} \\ \mathrm{k}+3: & 4800 \mathrm{M}^{-1} \mathrm{~s}^{-1}\end{array}$

\section{Error}

$+/-5.60 \mathrm{e}-005 \mathrm{M}^{-1} \mathrm{~s}^{-1}$

$+/-5.79 \mathrm{e}-005 \mathrm{~s}^{-1}$

$+/-1.19 \mathrm{e}-004 \mathrm{M}^{-1} \mathrm{~s}^{-1}$

$+/-1.34 \mathrm{e}-007 \mathrm{~s}^{-1}$

$+/-7.31 \mathrm{e}-005 \mathrm{M}^{-1} \mathrm{~s}^{-1}$
C

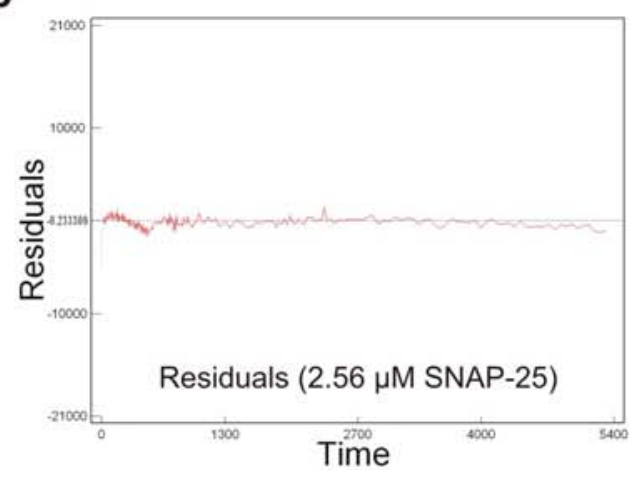

d

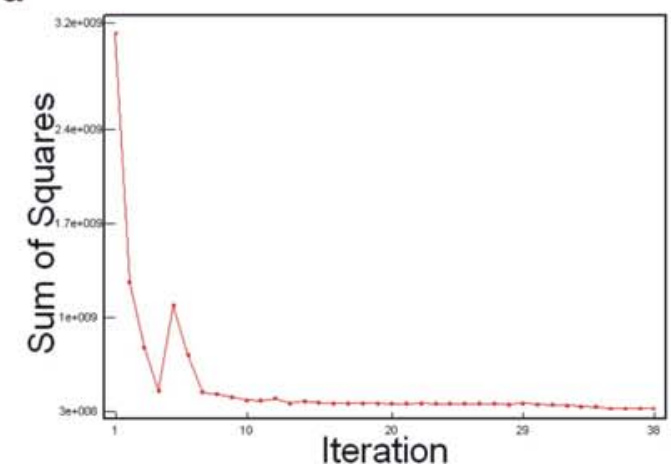


Figure 16. Simulation followed by fitting of the kinetic traces obtained in the SNAP-25 titration experiment, (FRET between $\mathrm{Syb}^{61 \mathrm{OG}} / \mathrm{Syx}^{225 \mathrm{TR}}$ ).

(experimental traces were the ones shown in figure 15a). The simulations and fitting were done essentially as described in the Methods. Note that whereas the $\mathrm{k}_{\text {on }}$ and $\mathrm{k}_{\text {off }}$ for the syntaxin/SNAP$251: 1$ acceptor complex remains the same as was determined for the soluble proteins (ref), the binding of synaptobrevin to the acceptor complex was retarded.

a) Plots of experimental traces with the fitted data. Red trace represents experimental trace, black trace represents fitted trace.

b) Kinetic model that was used for simulation and the $\mathrm{k}_{\text {on }}$ and $\mathrm{k}_{\text {off }}$ of reaction intermediates and product obtained from the fitting represented in the model as $\mathrm{k}+1, \mathrm{k}-1, \mathrm{k}+2, \mathrm{k}-2$ and $\mathrm{k}+3$.

c) Representative trace of residuals for the condition in which the concentration of SNAP-25 was $2.56 \mu \mathrm{M}$. Notice that the residuals trace fluctuates randomly around zero

d) The sum squares of residuals plotted against the number of iterations.

The determination of two sets of reaction rates was possible, depending on the starting values assigned to the formation and reaction rate constants before the iterative process was initiated. In the first fitting regime, using experimental data from titrations of SNAP-25 with the FRET pair Syb ${ }^{610 G}$ and Syx ${ }^{225 T R}$, the $\mathrm{k}_{\text {on }}$ and $\mathrm{k}_{\text {off }}$ for the 1:1 complex between syntaxin and SNAP-25 were calculated to be approximately $1900 \mathrm{M}^{-1} \mathrm{~s}^{-1}$ and $0.0007 \mathrm{~s}^{-1}$ respectively. The $\mathrm{K}_{\text {on }}$ for the $2: 1$ complex between syntaxin and SNAP-25 was calculated to be $250 \mathrm{M}^{-1} \mathrm{~s}^{-1}$ whereas that of binding of synaptobrevin in liposomes to the 1:1 syntaxin/SNAP-25 acceptor complex was approximately $4800 \mathrm{M}^{-1} \mathrm{~s}^{-1}$ (Fig. 16b). In this solution, it is noteworthy that the formation of the ternary SNARE complex is favoured over the unproductive 2:1 complex between syntaxin and SNAP-25 by a factor of 20. The concentration profiles of the reaction intermediate species suggests that the $1: 1$ syntaxin/SNAP-25 acceptor complex is present in large excess over the 2:1 syntaxin/ SNAP-25 intermediate (not shown). An important conclusion of this solution was that the binding of synaptobrevin to the syntaxin/SNAP-25 complex is very slow $\left(4800 \mathrm{M}^{-1} \mathrm{~s}^{-1}\right)$. This solution may be flawed since the experimentally determined values of $K_{\text {on }}$ and $k_{\text {off }}$ for the $\operatorname{syntaxin}_{2} / \mathrm{SNAP}-25$ complex do not agree with that of this solution. 
a

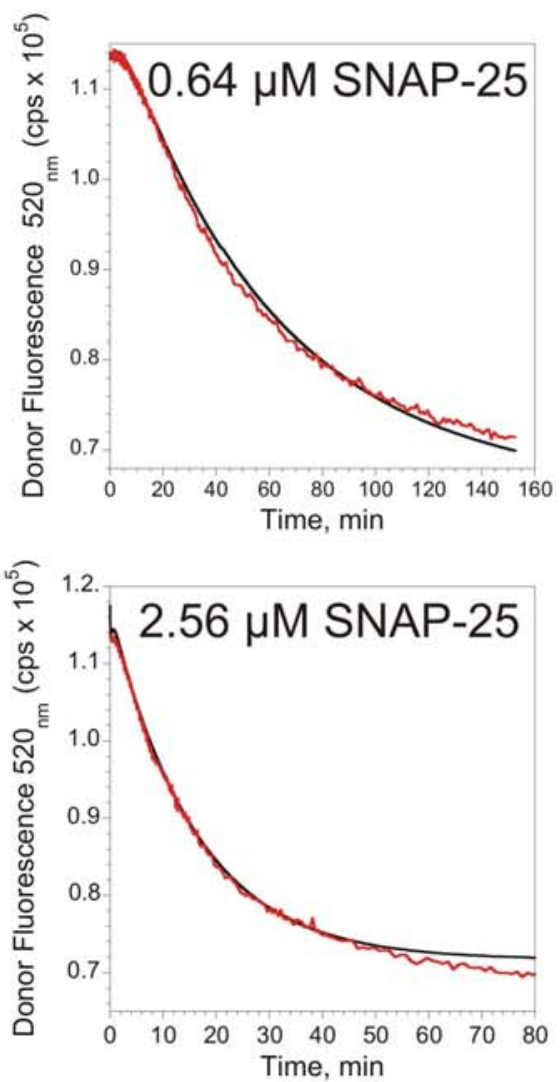

b

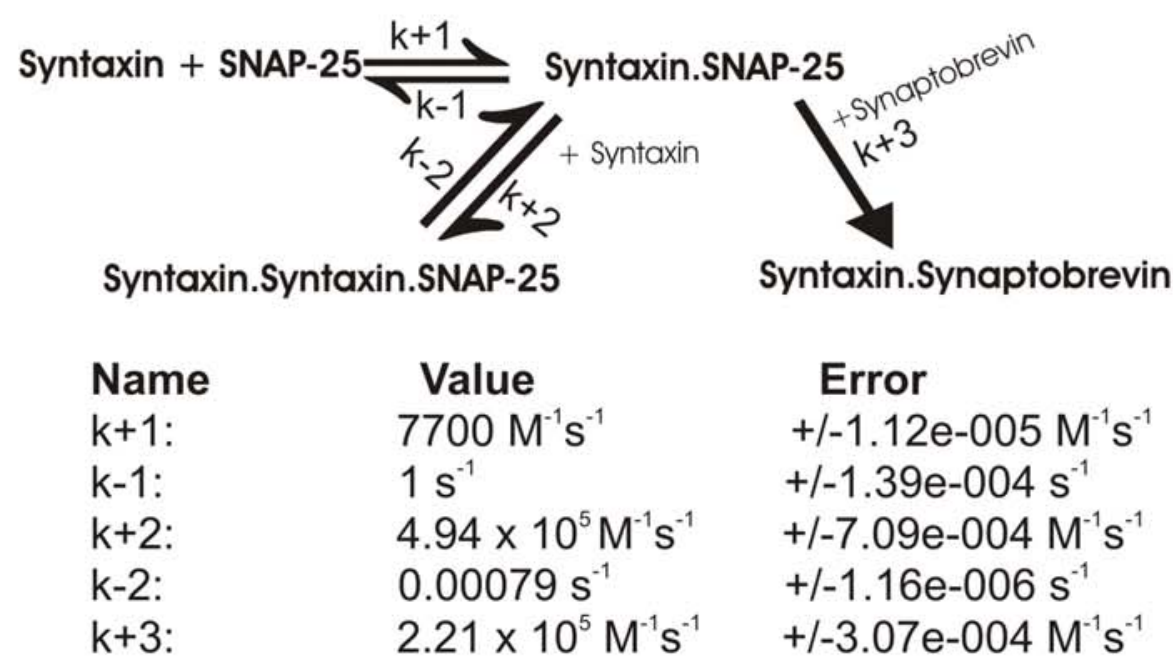

C



d

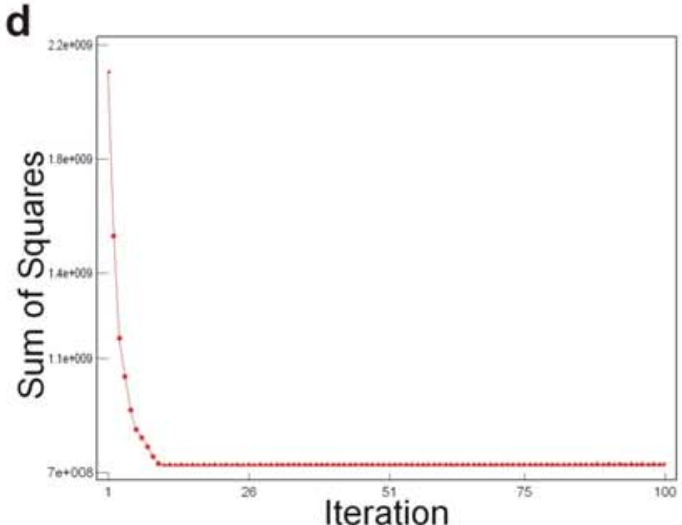


Figure 17. Simulation followed by fitting of the kinetic traces obtained in the SNAP-25 titration experiment (FRET between Syb61OG/Syx225TR).

(experimental traces were the ones shown in figure 15a). The simulations and fitting were done essentially as described in the Methods. Note that whereas the $k_{\text {on }}$ for the syntaxin/SNAP-25 1:1 acceptor complex remains the same as was determined for the soluble proteins (ref), $\mathrm{k}_{\text {off }}$ for this complex was about 100 times higher than that determined for the soluble proteins. Notice that the binding of synaptobrevin to the acceptor complex was rapid.

a) Plots of experimental traces with the fitted data. Red trace represents experimental data, black trace represents fitted data.

b) Kinetic model that was used for simulation and the $\mathrm{k}_{\text {on }}$ and $\mathrm{k}_{\text {off }}$ of reaction intermediates and product obtained from the fitting represented in the model as $\mathrm{k}+1, \mathrm{k}-1, \mathrm{k}+2, \mathrm{k}-2$ and $\mathrm{k}+3$.

c) Representative trace of residuals for the condition in which the concentration of SNAP-25 was $2.56 \mu \mathrm{M}$. Notice that though the goodness of the fit was within the error margin, the residuals were not uniformly spaced around zero, particularly at the tail end of the fit.

d) The sum squares of residuals plotted against the number of iterations. 
a
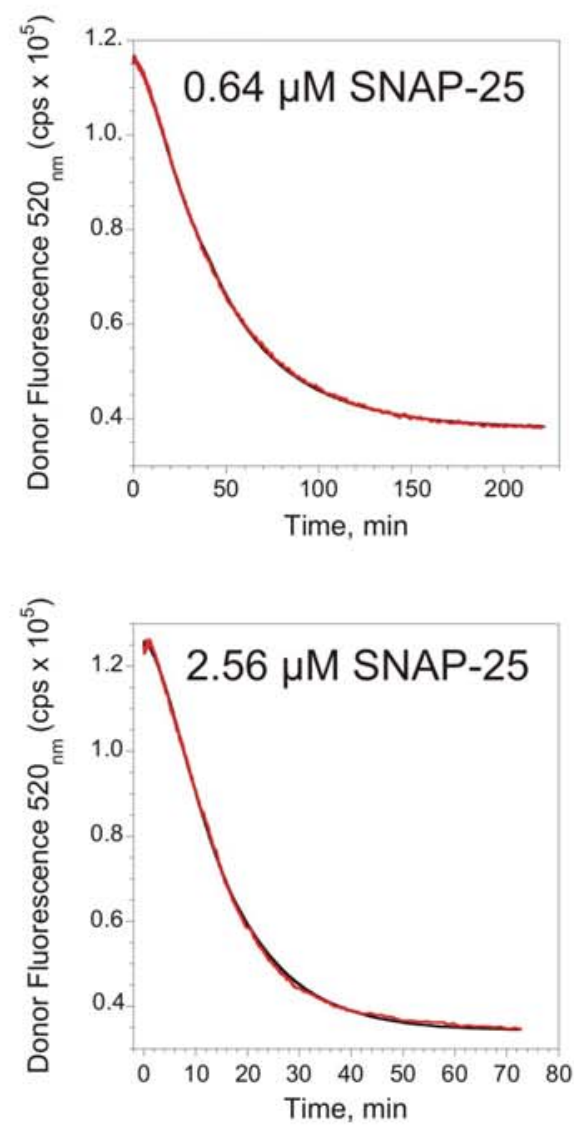
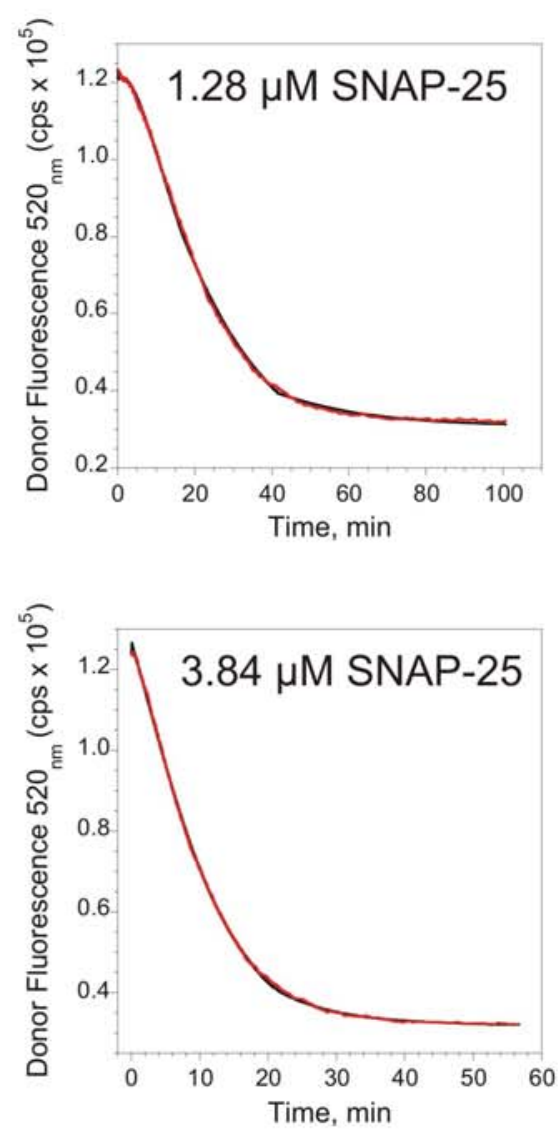

b



Syntaxin.Syntaxin.SNAP-25

Syntaxin.Synaptobrevin.SNAP-25

$\begin{array}{lll}\text { Name } & \text { Value } & \text { Error } \\ \text { k+1: } & 1260 \mathrm{M}^{-1} \mathrm{~s}^{-1} & +/-8.30 \mathrm{e}-005 \mathrm{M}^{-1} \mathrm{~s}^{-1} \\ \mathrm{k}-1: & 0.99 \mathrm{~s}^{-1} & +/-4.30 \mathrm{e}-002 \mathrm{~s}^{-1} \\ \mathrm{k}+2: & 2.75 \times 105 \mathrm{M}^{-1} \mathrm{~s}^{-1} & +/-8.99 \mathrm{e}-003 \mathrm{M}^{-1} \mathrm{~s}^{-1} \\ \mathrm{k}-2: & 0.0024 \mathrm{~s}^{-1} & +/-4.81 \mathrm{e}-005 \mathrm{~s}^{-1} \\ \mathrm{k}+3: & 1.79 \times 105 \mathrm{M}^{-1} \mathrm{~s}^{-1} & +/-8.10 \mathrm{e}-002 \mathrm{M}^{-1} \mathrm{~s}^{-1}\end{array}$

C

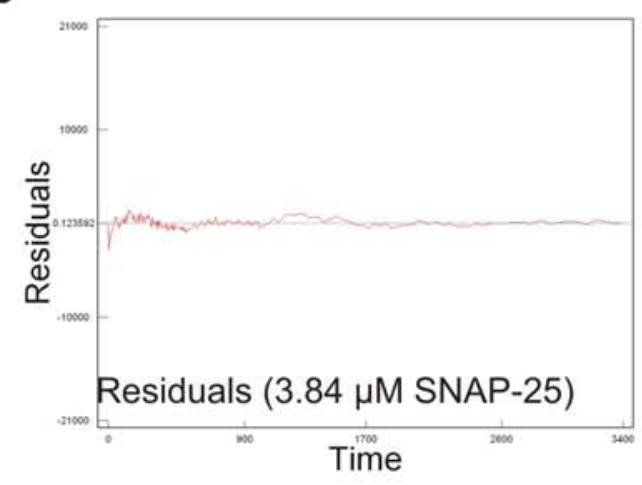

d




Figure 18. Simulation followed by fitting of the kinetic traces obtained in the SNAP-25 titration experiment (FRET between Syb28OG/Syx197TR).

(experimental traces were the ones shown in figure 15c). (see legend to figure 17 for details). Notice that the residuals trace fluctuates randomly around zero (c).

In the second solution, experimental data from titrations of SNAP-25 with two FRET pairs was used. The simulated traces from the fitting of data using the FRET pair $\mathrm{Syb}^{61 \mathrm{OG}}$ and $\mathrm{Syx}{ }^{225 \mathrm{TR}}$ result in $7700 \mathrm{M}^{-1} \mathrm{~s}^{-1}$ and $1 \mathrm{~s}^{-1}$ for $\mathrm{k}_{\text {on }}$ and $\mathrm{k}_{\text {off }}$ respectively for the $1: 1$ complex between syntaxin and SNAP-25. The $\mathrm{k}_{\text {on }}$ and $\mathrm{K}_{\text {off }}$ for the binding of second syntaxin molecule to the 1:1 syntaxin/SNAP-25 acceptor complex were $4.94 \times 10^{5} \mathrm{M}^{-1} \mathrm{~s}^{-1}$ and $0.00079 \mathrm{~s}^{-1}$ respectively, whereas the $\mathrm{k}_{\mathrm{on}}$ for the binding of synaptobrevin in liposomes to the 1:1 syntaxin/SNAP-25 acceptor complex was $2.21 \times 10^{5} \mathrm{M}^{-1} \mathrm{~s}^{-1}$ (Fig. 17b). The rates calculated for the experimental data using the FRET pair $\mathrm{Syb}^{280 G}$ and Syx ${ }^{197 \mathrm{TR}}$ were $1260 \mathrm{M}^{-1} \mathrm{~s}^{-1}$ and $0.99 \mathrm{~s}^{-1}, 2.75 \times 10^{5} \mathrm{M}^{-1} \mathrm{~s}^{-1}, 0.0024 \mathrm{~s}^{-1}$ and $1.79 \times 10^{6} \mathrm{M}^{-1} \mathrm{~s}^{-1}$ for $\mathrm{k}_{+1}, \mathrm{k}_{-1}, \mathrm{k}_{+2}, \mathrm{k}_{-2}$ and $\mathrm{k}_{+3}$ respectively (Fig. 18b). Two essential outcomes of the analysis using the second solution were that the syntaxin/SNAP-25 1:1 complex on membranes is highly unstable and the binding of synaptobrevin to the 1:1 syntaxin/SNAP-25 complex is extremely rapid.

Thus, two solutions of reaction rates for the intermediates and product were arrived at. The first solution suggested that the formation and dissociation of syntaxin/SNAP-25 1:1 acceptor is similar to that of the published values for soluble SNAREs (Fasshauer and Margittai, 2004). The syntaxin $_{2} /$ SNAP-25 binary complex was, however, strongly suppressed during the reaction. Finally, the binding of synaptobrevin to syntaxin/SNAP-25 was considerably slow. The second solution indicated that the $\mathrm{K}_{\text {on }}$ for syntaxin/SNAP-25 and $\operatorname{syntaxin}_{2} /$ SNAP-25 were comparable to that of the soluble proteins. The binding rate of synaptobrevin to the syntaxin/SNAP-25 acceptor complex, however, was rapid (on the order of $10^{5}$ to $10^{6} \mathrm{M}^{-1} \mathrm{~s}^{-1}$ ). The overall slow rate was traced to the high $\mathrm{K}_{\text {off }}$ for syntaxin/SNAP-25 complex (about 100 times faster than that estimated for proteins in solution). New experiments can be designed to test either possibility. 


\subsubsection{Substitution of membrane-proximal tryptophan residues by serine does not enhance the rate of complex formation}

The two highly conserved tryptophan residues at the C-terminal end of the SNARE motif of synaptobrevin were reported to cause membrane-insertion of the linker connecting the SNARE motif and the transmembrane domain (Kweon et al., 2003a; Kweon et al., 2003b). These authors reported that synaptobrevin reconstituted in liposomes entered into complexes only when the tryptophan residues are replaced with serines (Kweon et al., 2003b). The possibility that these tryptophan residues, by causing insertion into the lipid bilayer of the membrane-proximal stretch of synaptobrevin (aa 85-92) compromises its reactivity can be ruled out since the results above show that wild-type synaptobrevin in liposomes is fully capable of binding to syntaxin and SNAP25. It is possible though that when the proposed membrane-block is relieved by replacing the membrane-proximal tryptophan residues with smaller polar residues like serine, the rate of complex formation can be significantly enhanced. To assess this possibility, synaptobrevin W89S W90S was generated and reconstituted into proteoliposomes. Addition of soluble syntaxin and SNAP-25 led to the formation of SDS resistant ternary complex that dissociated into its components upon heating (Fig. 19a), suggesting that the mutant version of synaptobrevin is capable of engaging in SNARE complexes. Complex formation was also monitored by fluorescence anisotropy as described above. When soluble syntaxin and SNAP-25 were added to synaptobrevin W89S,W90S ${ }^{61 O G}$ reconstituted in liposomes, fluorescence anisotropy increased, denoting complex formation, which was effectively blocked by soluble unlabelled synaptobrevin (Fig. 19b). Complex formation rate was monitored in more detail by FRET using the FRET pair $\mathrm{Syb}^{61 \mathrm{OG}}$ and $\mathrm{Syx}^{225 \mathrm{TR}}$. As shown in Fig. 19c, the rate of complex formation was not accelerated with respect to the wild-type protein. Similar results were obtained when other labeling positions were used (not shown). The rate of complex formation increased when the SNAP-25 concentration was increased, as was shown for wild-type synaptobrevin (Fig. 19d), suggesting that the assembly pathway of synaptobrevin W89S,W90S in liposomes is simlar to that of the wild-type protein. 
a

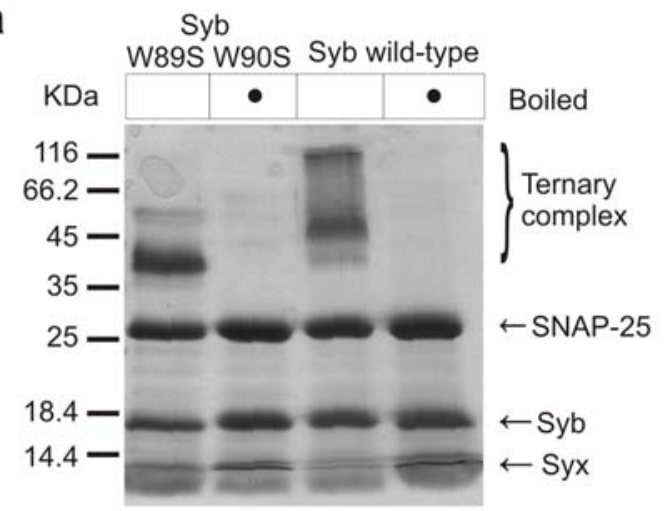

C

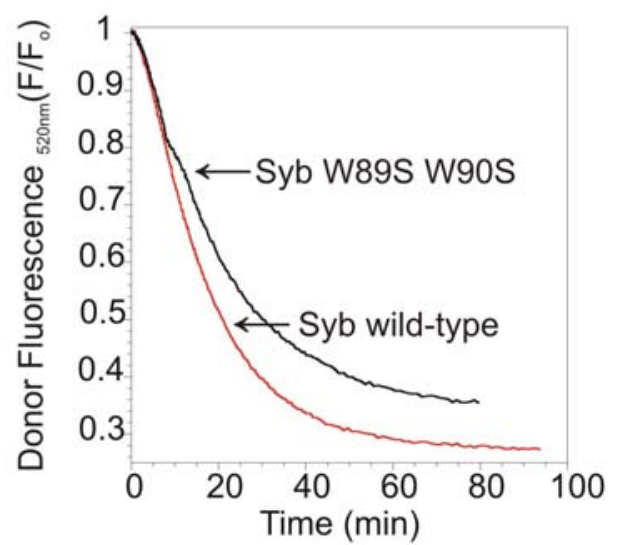

b

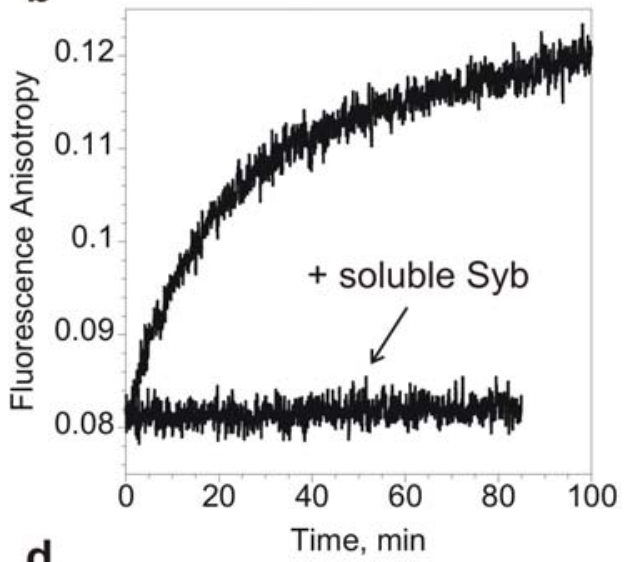

d

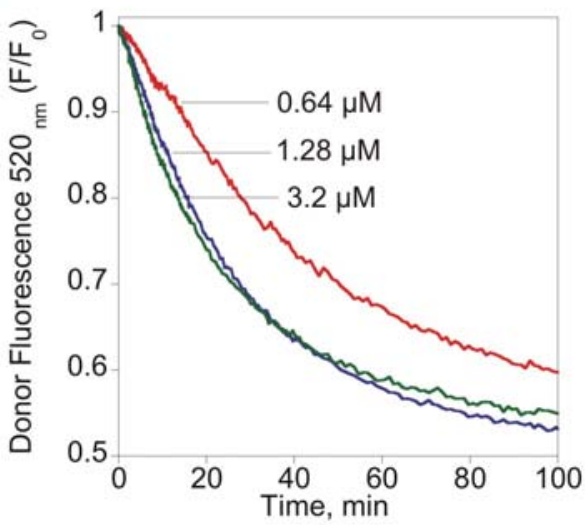

Figure 19. Substitution of the membrane proximal tryptophans 89 and 90 with serine does not alter the efficiency of synaptobrevin to enter SNARE complexes.

a) Complex formation, monitored by the appearance of heat-resistant bands after SDSPAGE and Coomassie Blue staining. Liposomes were reconstituted with either mutant or wild-type synaptobrevin (incubations as in Fig. 8, with approx. 100 and 200 pmoles of syntaxin and SNAP-25 respectively). Note that the complex containing synaptobrevin W89S W90S migrates somewhat faster in SDS-PAGE, probably indicating a difference in the amount of bound SDS.

b) Complex formation monitored by fluorescence anisotropy. Anisotropy increased when unlabeled soluble syntaxin $(1.3 \mu \mathrm{M})$ and SNAP-25 $(2.85 \mu \mathrm{M})$ were added to the reaction containing $\mathrm{Syb}^{61 \mathrm{OG}}$ W89S, W90S in liposomes $(150 \mathrm{nM})$. Note that excess soluble synaptobrevin $(5 \mu \mathrm{M})$ blocks the increase in anisotropy

c) Complex formation, monitored by FRET using the FRET pair $\mathrm{Syb}^{61 \mathrm{OG}} / \mathrm{Syx}^{225 \mathrm{TR}}$ (see legend to Figure 11a). Upon addition of SNAP-25 $(\sim 1 \mu \mathrm{M})$, complex formation on liposomes containing either variant of synaptobrevin proceeded at similar rates indicating that the mutation of membrane proximal tryptophans to serines does not accelerate the reaction rate.

d) Increasing concentrations of SNAP-25 profoundly accelerates the rate of complex formation indicating that Syb W89S, W90S in liposomes binds to the syntaxin/SNAP-25 1:1 acceptor complex. Complex formation was monitored by FRET (see legend to Figure $15 \mathrm{a})$. 


\subsubsection{SNARE complex assembly rate is enhanced in the presence of weak counterions}

The rate of SNARE complex assembly on reconstituted synaptobrevin liposomes is very slow when compared to that of soluble SNAREs. It is conceivable that SNARE complex assembly on membranes is affected by intracellular factors. To test the possibility that intracellular factors may enhance complex assembly rate, the reaction was monitored by the FRET assay discussed above in the presence or absence of rat brain cytosol. No difference in assembly rate was observed (Fig. 20a). To preclude the possibility that the complex that formed was simultaneously disassembled by the NSF/ $\alpha$ SNAP machinery, NEM, a known inhibitor of NSF and ATP $\gamma$, the non-hydrolyzable form of ATP that binds to the ATP-binding site of NSF, were included in the reaction. The complex assembly rate did not differ from the control reaction suggesting that brain cytosolic proteins may not directly catalyze SNARE complex assembly (Fig. 20b).

a

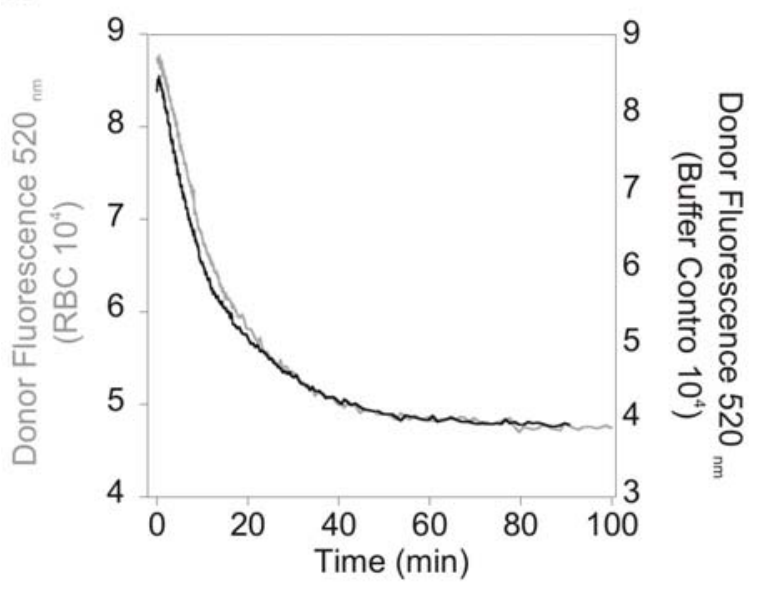

b

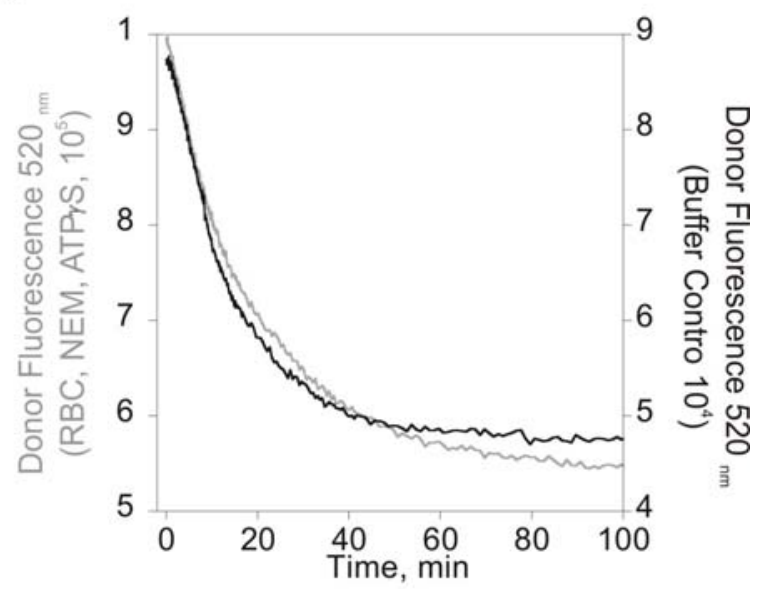

Figure 20. Rate of SNARE complex formation on synaptobrevin-containing liposomes is not changed by brain cytosolic proteins.

Complex formation was monitored by FRET (see legend to Figure 11a for details).

a) When Rat Brain Cytosol was added to the reaction cuvette to a final concentration of 1 $\mathrm{mg} / \mathrm{ml}$, complex formation was not enhanced.

b) To rule out the possibility that NSF/ $\alpha$-SNAP were disassembling the SNARE complexes formed, NEM (300 $\mu \mathrm{M}$ final conc.) and ATP $\gamma \mathrm{S}(400 \mu \mathrm{M}$ final conc.) were included in the reaction. No difference in the complex formation rate was observed. 
The crystal structure of the SNARE complex suggests two divalent co-ordinating sites in the groove between SNAP-25 and synaptobrevin (Sutton et al., 1998). The presence of calcium in the reaction may thus play a role in complex assembly rate. As shown in Fig. 21, using the fluorescence anisotropy assay described above, SNARE complex assembly rate was unaffected in the presence of $1 \mathrm{mM}$ calcium.

The lipid environment in a physiological setting may be different from the reduced system used in this study. Furthermore, a study suggested that SNAREs normally prefer liquid-disordered phase in the membrane (Bacia et al., 2004). The fluidity of the lipid bilayer could thus influence the rate of SNARE complex assembly. The fluidity of a bilayer being dependent on temperature (Canvin and Buhr, 1989), SNARE complex assembly was carried out at varying temperatures $\left(18^{\circ} \mathrm{C}, 25^{\circ} \mathrm{C}\right.$ and $\left.30^{\circ} \mathrm{C}\right)$. The rate of complex formation, however, remained unchanged, suggesting that the fluidity of membrane may have little or no bearing on the rate of assembly of SNAREs (Fig. 21).

The membrane-proximal region of synaptobrevin (aa 77-90) has been reported to bind to liposomes containing acidic phospholipids but not to liposomes containing only non-charged phospholipids (Quetglas et al., 2000). This finding raises the possibility that binding of syntaxin/SNAP-25 to synaptobrevin reconstituted in liposomes containing only neutral lipids might influence the latter's reactivity. However, in fluorescence anisotropy experiments to report complex formation, SNARE complex formation rate with synaptobrevin reconstituted in neutral lipids (PC:Cholesterol: 70:30) remained unchanged (Fig. 21). 




Figure 21. Rate of SNARE complex assembly does not depend on extraneous conditions.

SNARE complex assembly was monitored by fluorescence anisotropy. Upon addition of SNAP$25(1.92 \mu \mathrm{M})$ to syntaxin $(1.25 \mu \mathrm{M})$ and $\mathrm{Syb}^{280 \mathrm{G}}$ in liposomes $(125 \mathrm{nM})$, an increase in anisotropy was observed. Complex formation rate did not alter if the temperature was raised to $30^{\circ} \mathrm{C}$ or lowered to $18^{\circ} \mathrm{C}$. When $\mathrm{Syb}^{280 \mathrm{G}}$ was reconstituted in liposomes composed of neutral lipids (phospatidycholine/cholesterol in a ratio of 70:30) or divalent ions $\left(\mathrm{Ca}^{+2}\right.$, final conc. $1 \mathrm{mM}$ ), complex formation rate was not altered, indicating that SNARE complex formation is robust and relatively independent of exogenous factors.

a



b

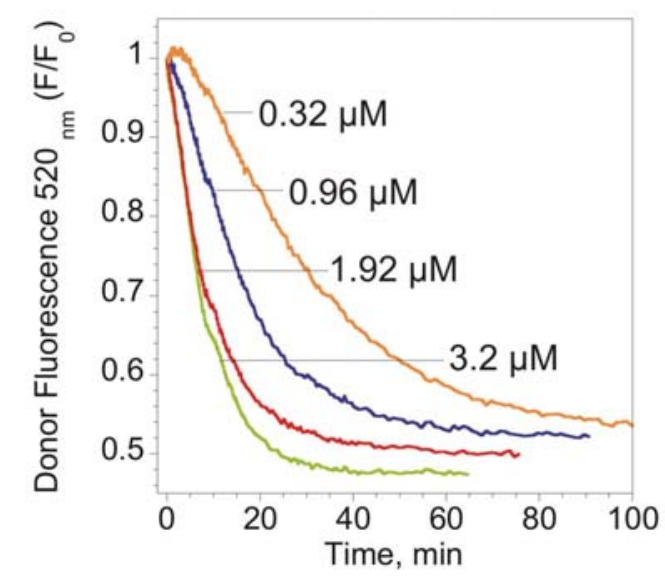

Figure 22. SNARE complex formation on synaptobrevin (Syb)-containing liposomes with a lipid content of POPC:DOPS in a ratio of 85:15, monitored by FRET.

a) FRET between $\mathrm{Syb}^{610 \mathrm{G}}$ and $\mathrm{Syx}^{225 \mathrm{TR}}$. $\mathrm{Syb}^{61 \mathrm{OG}}$, reconstituted in liposomes (POPC:DOPS in a ratio of 85:15) ( $100 \mathrm{nM}$ final conc.) was incubated with Syx ${ }^{225 T R}$ (final conc. $\sim 300$ $\mathrm{nM})$. Upon addition of SNAP-25 $(1.92 \mu \mathrm{M})$ donor fluorescence decreased, indicating complex formation. To test the specificity of signal change, the proteoliposomes were preincubated with trypsin as described in Fig. 11c. FRET did not occur in trypsin treated synaptobrevin liposomes. 
b) Increasing concentration of SNAP-25 increased the rate of complex formation (see legend to Fig. 15), indicating SNARE complex assembly pathway remains unchanged when synaptobrevin is inserted in liposomes of different lipid compositions.

Kweon et al. did not observe SNARE complex assembly on membranes in studies using Electron paramagnetic resonance (Kweon et al., 2003b). The lipid composition of the liposomes used in their study was POPC:DOPS in a ratio of 85:15. To rule out the possibility that SNARE complexes do not form on liposomes composed of POPC/DOPS, proteoliposomes containing $\mathrm{Syb}^{61 \mathrm{OG}}$ were generated. When reconstituted synaptobrevin was incubated with soluble syntaxin and SNAP-25, SNARE complexes formed, as assessed by FRET (Fig. 22). When the proteoliposomes were pre-treated with trypsin, complex formation was blocked. As described above, the ternary complex transits through the 1:1 acceptor complex between syntaxin and SNAP-25. As per the model, excess of SNAP-25 enhances the rate of assembly on synaptobrevin in POPC/DOPS liposomes (Fig. 22), indicating that the ability of synaptobrevin incorporated in liposomes of different lipid compositions to engage soluble syntaxin and SNAP-25 in a complex is the same. Therefore, divalent ions, membrane fluidity, lipid composition and intracellular factors do not appear to enhance or mitigate the rate of SNARE complex assembly. Is SNARE complex assembly then dependent on extraneous physical parameters like chaotropicity, ionic strength or the presence of weak counter-ions? To investigate these possibilities, SNARE complex assembly under different solution compositions was monitored by the FRET assay. When the salt in the solution was changed from $150 \mathrm{mM}$ $\mathrm{NaCl}$ to $140 \mathrm{mM} \mathrm{KCl}$ or $140 \mathrm{mM} \mathrm{KI}$, no difference in the reaction rate was discernible, whereas the absence of ionic charges greatly retarded the reaction rate (Fig. 23a). When $140 \mathrm{mM} \mathrm{K}$-Glutamate/20 mM K-Acetate was used as the salts in the solution, the reaction rate was significantly enhanced (Fig. 23a). Complex formation of soluble SNAREs too was monitored in the presence of $150 \mathrm{mM} \mathrm{NaCl}$ or $140 \mathrm{mM} \mathrm{K}$-Glutamate/20 mM KAcetate (Fig. 23b). The rate of complex assembly was higher in the K-Glutamate/KAcetate solution indicating that the presence of weak counterions at the concentrations specified was the sole criteria for the enhancement of SNARE complex assembly rate, a condition known to stabilize protein-protein interaction in general (Griep and McHenry, 1989; Reichert and Moore, 2000; Zou and Richardson, 1991). 
a

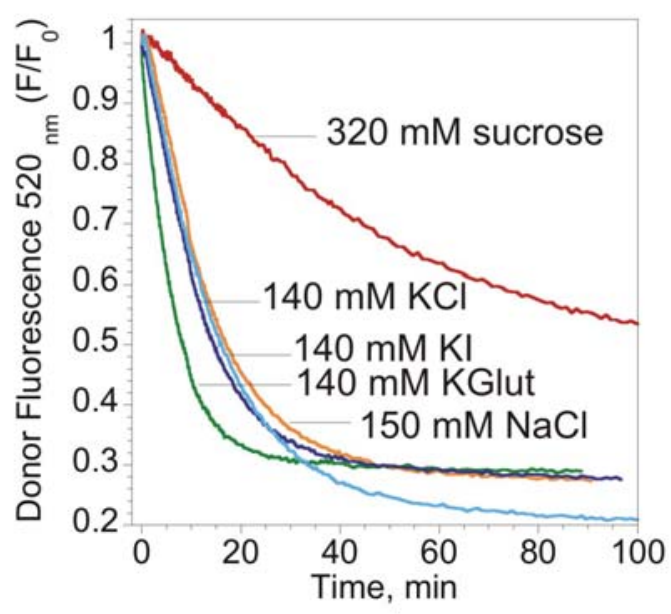

b

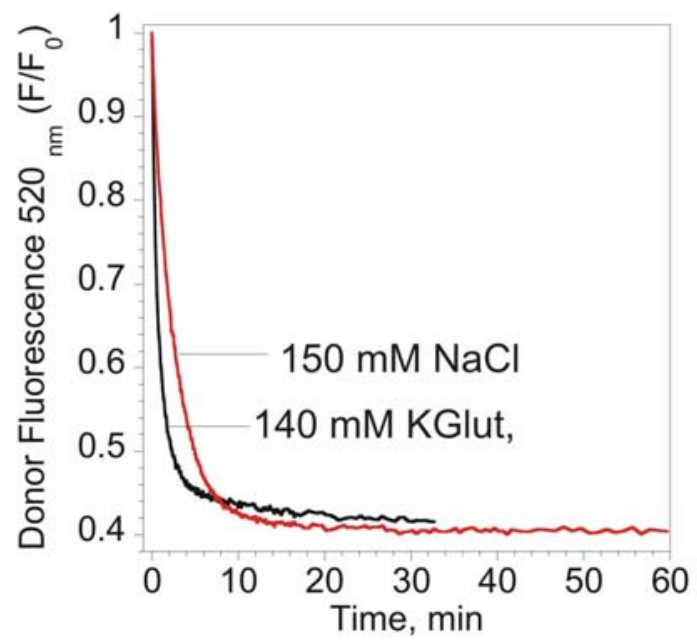

Figure 23. Rate of SNARE complex assembly on synaptobrevin-containing liposomes in the presence of different solvents.

(monitored by FRET between $\mathrm{Syb}^{61 \mathrm{OG}} / \mathrm{Syx}^{225 \mathrm{TR}}$ as described in Fig. 11a except that the concentration of SNAP-25 was $1.92 \mu \mathrm{M}$ )

a) SNARE complex assembly rate in the presence of $\mathrm{KCl}, \mathrm{NaCl}$ and $\mathrm{KI}$ was unaltered. It was retarded in the presence of sucrose and enhanced in the presence of K-Glut $/ \mathrm{K}$ Acetate, indicating that whereas the presence of weak counterions is sufficient to enhance the rate of complex formation, the absence of ions can abolish assembly.

b) SNARE complex assembly rate of souble $\operatorname{Syb}^{610 G}(150 \mathrm{nM}) \mathrm{Syx}^{225 \mathrm{TR}}(500 \mathrm{nM})$ and SNAP-25 $(1.28 \mu \mathrm{M})$ in presence of $150 \mathrm{mM} \mathrm{NaCl}$ or $140 \mathrm{mM} \mathrm{K}$-Glut/20 mM K-Acetate, indicating that the even the soluble proteins form SNARE complexes faster in the presence of weak counter-ions.

\subsection{SNARE complex assembly on synaptic vesicles}

The results described so far show that synaptobrevin retains its ability to form SNARE complexes after insertion into liposomes. It is possible, however, that in its native environment, the reactivity of synaptobrevin is downregulated by interaction with other proteins. I therefore investigated whether synaptobrevin in synaptic vesicles can also engage in SNARE complexes. In synaptic vesicles purified from rat brains, almost the entire pool of synaptobrevin is sensitive to digestion by tetanus toxin light chain (Fig. 24a, left), indicating its total outward orientation. Addition of SNAP-25 and syntaxin quantitatively shifted the synaptobrevin into an SDS-resistant band of higher molecular weight, which, as expected for SNARE core complexes, was insensitive to toxin digestion (Fig. 24a, right). Similar results were obtained when the soluble domain of syntaxin with the intact $\mathrm{N}$-terminal domain was used instead of the syntaxin with only the SNARE motif (Fig. 24b). 
As an independent indicator of SNARE complex assembly on synaptic vesicles, the FRET assay was used. Since synaptobrevin in the vesicle cannot be selectively labelled with a fluorescent dye, an indirect approach was employed to monitor ternary SNARE complex. The off-pathway during the assembly of ternary SNARE complex is the formation of the $\operatorname{syntaxin}_{2}$ :SNAP-25 binary complex. The $t_{1 / 2}$ for the dissociation of the second syntaxin molecule was determined to be between 400 and 1400 seconds in the models discussed above (see Figs. 17 and 18), leading to the transitory syntaxin/SNAP-25 acceptor complex to which a synaptobrevin molecule can readily bind. The formation of the ternary complex is essentially an irreversible reaction. Hence, the addition of synaptobrevin to the $\operatorname{syntaxin}_{2}$ :SNAP-25 reaction would result in the irreversible replacement of one of the syntaxin molecules and provide a ready binding site for synaptobrevin. The $\operatorname{syntaxin}_{2}$ :SNAP-25 complex was generated by incubating equimolar amounts of SNAP-25 and syntaxin labelled at position 225 with either Oregon Green or Texas Red. Since the two syntaxin and the lone SNAP-25 molecule have a parallel arragment (Margittai et al., 2001; Xiao et al., 2001), the two syntaxin molecules were therefore in a state in which the donor fluorescence was quenched. Increasing amounts of synaptic vesicles caused faster irreversible formation of ternary complex, as assessed by donor de-quenching (Fig. 24c) or acceptor fluorescence increase (Fig. 24d). These experiments lend support to the conclusion that synaptobrevin resident on synaptic vesicles is fully active. 
a

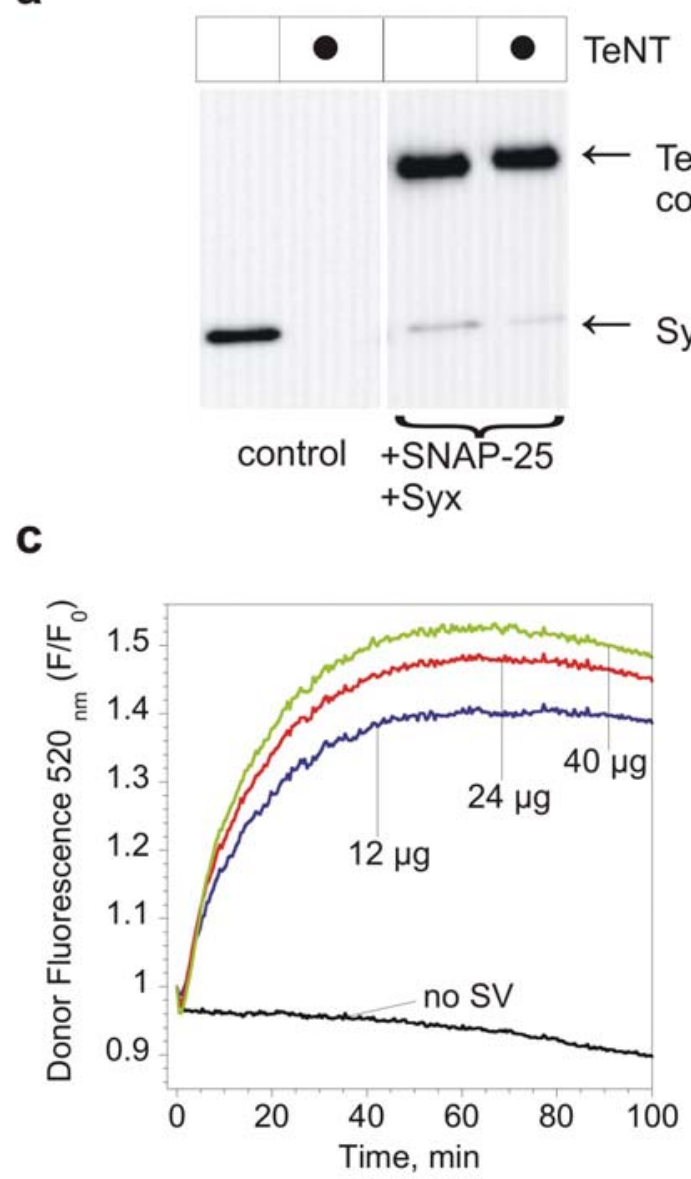

b



d

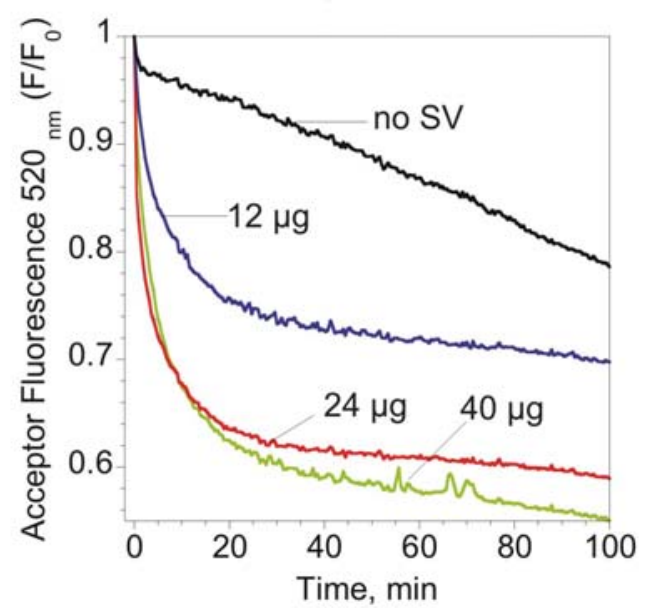

Figure 24. SNARE complex assembly on synaptic vesicles (SV).

a) SNARE complex formation on synaptic vesicles, monitored by SDS-PAGE followed by immunoblotting for synaptobrevin. Syntaxin (40 pmoles) and SNAP-25 (100 pmoles) were incubated with $5.6 \mu \mathrm{g}$ of purified synaptic vesicles for $4 \mathrm{~h}$ in $50 \mu \mathrm{l}$ volume. The reactions were stopped by $50 \mu 12 \mathrm{x}$ sample buffer containing SDS; $5 \mu 1$ of this reaction was analyzed. Complex formation on synaptic vesicles resulted in an almost complete shift of synaptobrevin to an SDS-resistant band of higher molecular weight (Ternary complex). Treatment of vesicles with the light chain of tetanus toxin (TeNT) before addition of SNAP-25 and syntaxin cleaved nearly the entire pool of synaptobrevin (left lanes), documenting that it is completely accessible.

b) Similar experiment as in a) except that syntaxin with the intact cytoplasmic domain (aa 1262) was used. Almost the entire pool of synaptobrevin on synaptic vesicles forms a ternary complex with the intact cytoplasmic portion of syntaxin.

c) and d) SNARE complex assembly monitored by FRET. Syx ${ }^{225 T R}(100 \mathrm{nM})$ and $\operatorname{Syx}^{2250 G}(100 \mathrm{nM})$ were preincubated with SNAP-25 $(200 \mathrm{nM})$ for $1 \mathrm{~h}$ to allow for the formation of the syntaxin/SNAP-25 2:1 complex. Synaptobrevin replaces one of the syntaxins to form the ternary SNARE complex. Increasing the amount of synaptic vesicles increased the rate of displacement of one molecule of syntaxin from the syntaxin/SNAP-25 2:1 complex as seen by donor fluorescence increase and acceptor fluorescence decrease, thus indicating that soluble syntaxin and SNAP-25 can form complexes with synaptobrevin on synaptic vesicles. 


\subsubsection{SNARE complex formation on synaptic vesicles is not influenced by calcium}

Since synaptic vesicles contain the putative calcium sensor (synaptotagmin I), the rate of complex formation in the presence or absence of calcium ions was also tested. Synaptic vesicles were incubated with syntaxin and SNAP-25 for $30 \mathrm{~min}$, i.e., conditions under which complex formation was not complete. No difference was observed in the presence of calcium concentrations up to $1 \mathrm{mM}$ (Fig 25a.). Complex formation was also monitored on synaptic vesicles over time at various calcium concentrations. As shown in Fig. 25b, SNARE complexes increased over time at all concentrations of calcium indicated.

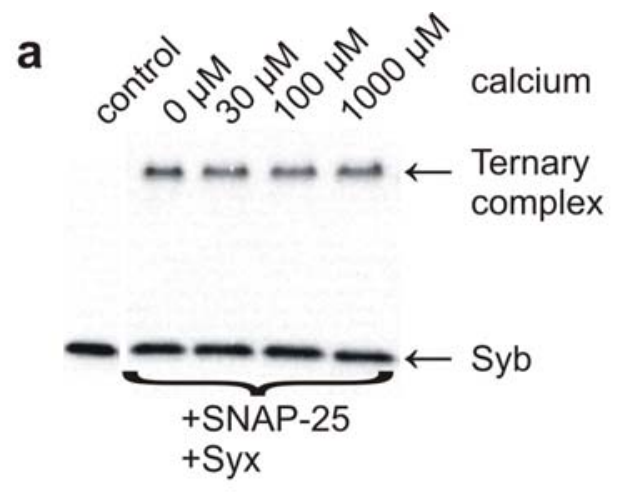

b

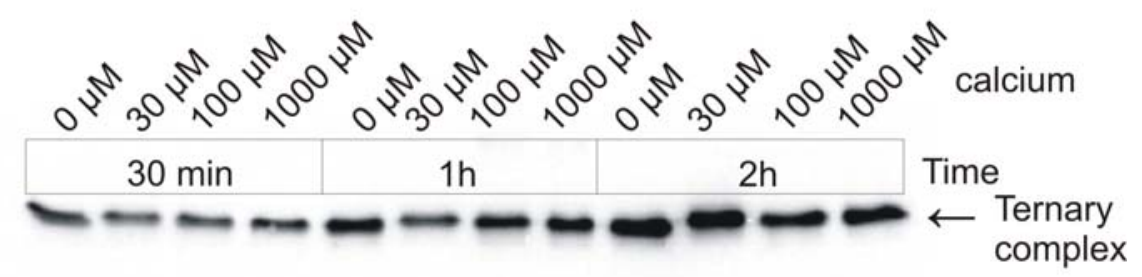

Figure 25. SNARE complex assembly on synaptic vesicles does not change in the presence of increasing amounts of calcium.

Conditions were the same as in Fig. 24a) except that reactions were carried out in the stated amounts of calcium. a) SNARE complex assembly monitored by immunoblotting for synaptobrevin. Synaptic vesicles were incubated with syntaxin and SNAP-25 for 30 minutes in the presence of the indicated $\mathrm{Ca}^{+2}$ concentrations. Note that under these conditions, complex formation is not completed (see also Fig. 26). b) SNARE complex assembly over time monitored by anti-ternary complex antibody (cl 135.2) indicating that complex formation increased over time, irrespective of the amounts of calcium in the reactions. 


\subsubsection{Reactivity of synaptobrevin in liposomes and synaptic vesicles is comparable}

In the next experiments, the reactivity of synaptobrevin in proteoliposomes and synaptobrevin in synaptic vesicles was compared. To make the reaction rates comparable, the amounts of synaptobrevin were matched using approximately twice as much for the generation of liposomes in order to account for random orientation. Since endogenous synaptobrevin cannot be labeled, the reaction was again monitored by the appearance of SDS-resistant SNARE complexes. For complex formation, a fluorescently labeled syntaxin variant $\left(\mathrm{Syx}^{225 \mathrm{OG}}\right)$ was used, allowing to monitor the reaction both by immunoblotting for synaptobrevin and by fluorescence. Addition of SNAP-25 and syntaxin to synaptobrevin-containing liposomes or synaptic vesicles, respectively, resulted in complex formation at comparable rates, regardless of whether immunoblotting or fluorescence was used as read-out (Fig. 26). 
Immunoblot anti-syb

$0^{\prime} 2^{\prime} 5^{\prime} 15^{\prime} 30^{\prime}$ ' $\mathrm{h}$ 2h $4 \mathrm{~h} 6 \mathrm{~h}$

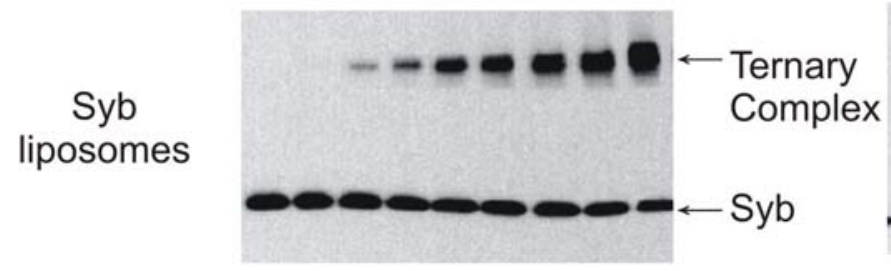

$0^{\prime} 2^{\prime} 5^{\prime} 15^{\prime} 30^{\prime}$ ih $2 \mathrm{~h}$ 4h $6 \mathrm{~h}$



Syx fluorescence

0' 2' 5' 15'30' 1h 2h 4h 6h

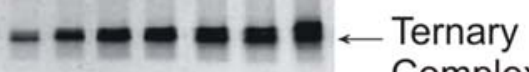

Complex

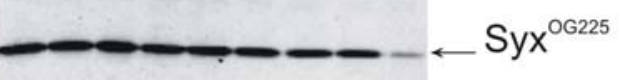

0' 2' 5' $15^{\prime} 30^{\prime}$ ih $2 \mathrm{~h}$ 4h $6 \mathrm{~h}$

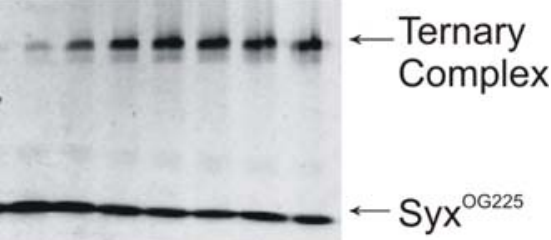

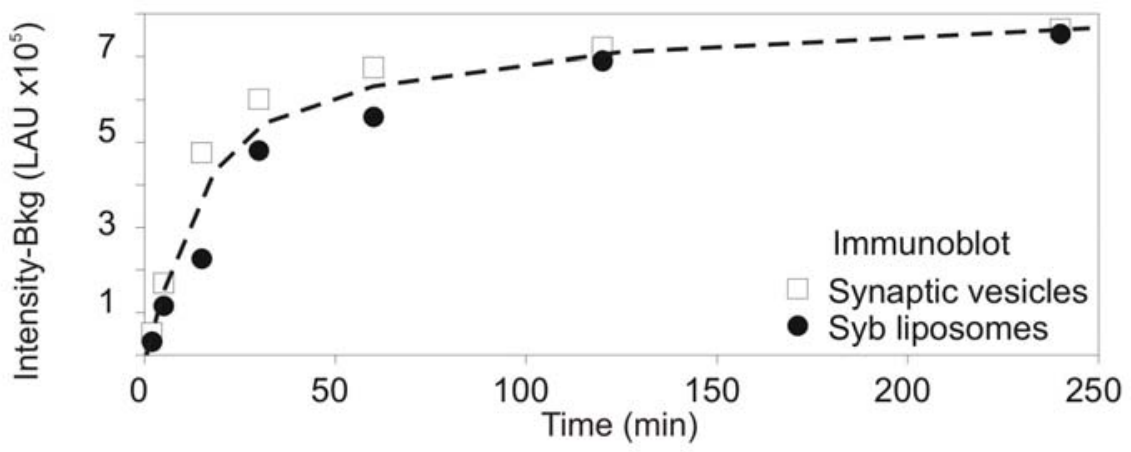

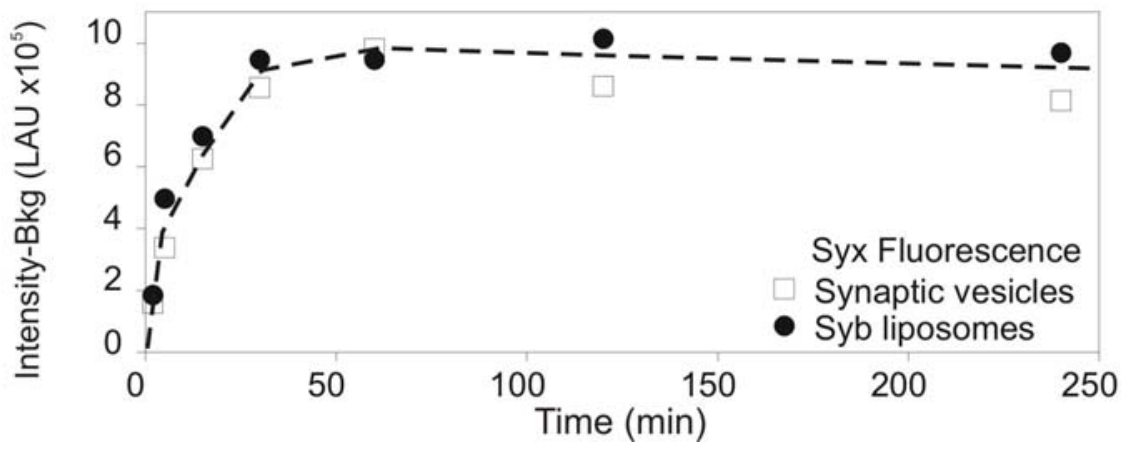

Figure 26. The rates of SNARE complex assembly on synaptic vesicles and on synaptobrevin liposomes are comparable.

$\mathrm{Syx}^{2250 \mathrm{G}}$ and SNAP-25 were incubated with synaptobrevin liposomes or synaptic vesicles (see Fig. 24a legend). SNARE complex formation, measured by the appearance of SDS-resistant bands, was monitored by immunoblotting for synaptobrevin (left panels) or by measuring fluorescence derived from syntaxin (right panels). To ensure that the reactions are completely arrested at the end of the incubation, SDS-containing sample buffer was added and the samples were immediately shock-frozen, and thawed only immediately prior to SDS-PAGE. For quantitation, the intensity of the bands was determined, corrected for background and plotted against incubation time. 


\subsection{Soluble syntaxin and SNAP-25 displace synaptobrevin from the synaptophysin/synaptobrevin heterodimer to form the ternary SNARE complex}

In synaptic vesicles, synaptobrevin is complexed with synaptophysin in a manner that is mutually exclusive with its interaction with SNAREs (Edelmann et al., 1995; Pennuto et al., 2002; Yelamanchili et al., 2005). The possibility that complex formation with exogenous SNAREs leads to a dissociation of synaptobrevin from synaptophysin was therefore tested. A fraction enriched in synaptic vesicles was incubated with SNAP25 and syntaxin, followed by detergent solubilization and immunoprecipitation of either synaptobrevin or synaptophysin. As shown in Fig. 27a, addition of the SNAREs caused a massive reduction in the amount of synaptobrevin co-precipitating with synaptophysin. Conversely, upon SNARE addition, a similarly strong reduction was observed in the amount of synaptophysin that co-precipitated with synaptobrevin (Fig. 27b). These results indicate that SNARE complex formation effectively dissociates synaptobrevin from synaptophysin. To confirm that synaptophysin does not interact with assembled SNARE complexes, complex formation was carried out using fluorescently labeled syntaxin, followed by solubilization and immunoprecipitation with synaptobrevin- or synaptophysin-specific antibodies, respectively. As shown in Fig. 27c, labelled syntaxin is only detectable in the synaptobrevin immunoprecipitates. As an independent read-out for the synaptophysin-synaptobrevin complex, which avoids detergent solubilization, I employed cross-linking using the bifunctional cross-linker DSS (Edelmann et al., 1995). Cross-linking resulted in the appearance of an additional band of approximately $55 \mathrm{kDa}$ that was positive for both synaptophysin (Fig. 27d) and synaptobrevin (not shown). Incubation of the vesicles with SNAP-25 and syntaxin prior to cross-linking prevented the formation of the adduct (Fig. 27d) suggesting that the endogenous synaptobrevin of synaptic vesicles is fully active with respect to SNARE complex formation and that it dissociates from synaptophysin when entering SNARE complexes. 



Figure 27. Synaptobrevin is displaced from synaptophysin (Syp) upon formation of SNARE complexes.

$\mathrm{a}, \mathrm{b}$ and $\mathrm{c}$ ) An enriched vesicle fraction (LP2, $50 \mu \mathrm{g}$ of protein) was incubated in the presence or absence of $50 \mu \mathrm{g}$ fluorescently labeled Syx ${ }^{225 \mathrm{TR}}, 200 \mu \mathrm{g}$ unlabelled syntaxin and $500 \mu \mathrm{g}$ SNAP-25 for $2 \mathrm{~h}$., followed by solubilization in Triton X-100 and immunoprecipitation for either synaptophysin (a) or synaptobrevin (b). All samples were analyzed by SDS-PAGE a) Immunoblotting for synaptobrevin shows that the amount of synaptobrevin coprecipitating with synaptophysin is reduced in the presence of syntaxin and SNAP-25

b) Conversely, immunoblotting for synaptophysin shows that the amount of synaptophysin coprecipitating with synaptobrevin is reduced in the presence of SNAREs. Note that in both cases the efficiency of antigen immunoprecipitation is comparable.

c) SNARE complexes (visualized by fluorescence of $\mathrm{Syx}^{225 \mathrm{TR}}$ ) coprecipitate with synaptobrevin but not with synaptophysin.

d) Disappearance of the synaptophysin-synaptobrevin complex in the presence of unlabelled syntaxin and SNAP-25, monitored by cross-linking with DSS, a bifunctional reagent. In the absence of the SNAREs, cross-linking results in the appearance of a band of $\sim 55 \mathrm{kDa}$ (*) that is recognized by both synaptophysin- (d) and synaptobrevin-specific (data not shown) antibodies and thus represents a heterodimer (Edelman).

IP: Immunoprecipitation, IB: Immunoblotting. 


\subsection{Characterisation of monoclonal antibodies against neuronal ternary SNARE complex}

Monitoring of SNARE complexes within a cell have been done by either overexpression studies involving fluorescently tagged SNARES (An and Almers, 2004) or by the use of toxins that clip individual SNAREs at specific sites, reviewed in (Montecucco et al., 2005). The second part of this work involved the characterisation of and subsequent assay development with, monoclonal antibodies that recognise the ternary SNARE complex but not the individual monomeric SNAREs. The antigen used for immunisation was the complex of synaptobrevin (aa 1-96), syntaxin (aa 180-262) and SNAP-25 first and second helices. Three antibodies were characterised: 135.1 (subtype IgG1), 135.2 (subtype $\operatorname{IgG} 2 b$ ) and 135.5 (subtype IgG1).

a

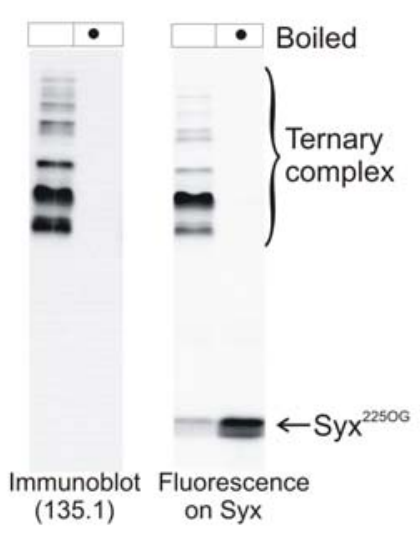

b

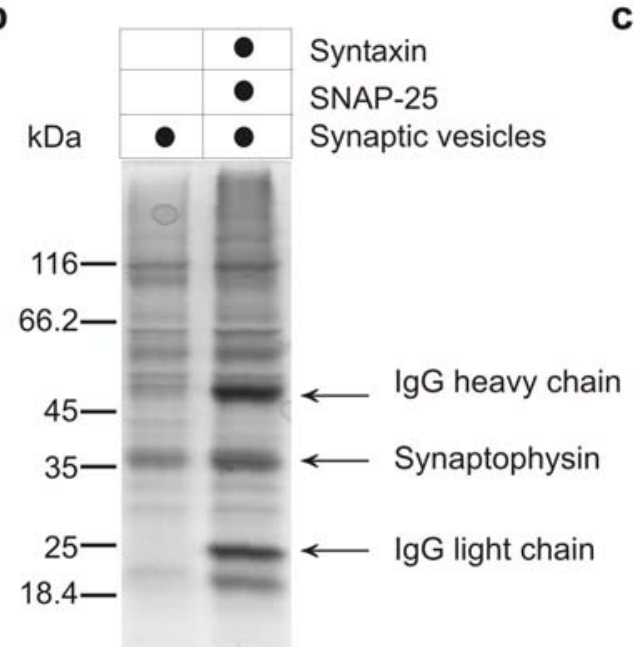

C

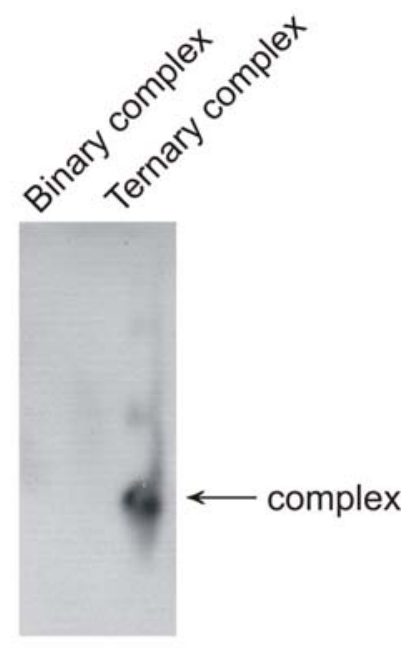

Figure 28. Binding specificity of anti-ternary complex antibodies to the SNARE complex.

a) Fluorescently labeled syntaxin $\left(\mathrm{Syx}^{225 \mathrm{OG}}\right.$ ), synaptobrevin (aa 1-96) and SNAP-25 were incubated in a molar ratio of 1:1:2 for $1 \mathrm{~h}$ before SDS-PAGE and immunobloting for ternary complex with anti-ternary complex antibodies. $\mathrm{Cl} 135.1$ recognizes only the ternary SNARE complexes and not the monomeric SNAREs, i.e., when the complex is boiled prior to loading (left). The bands corresponding to the tenary were also visible due to fluorescence on syntaxin (right). Boiled complex shows the fluorescently labeled monomeric syntaxin. Similar results were obtained with cl 135.2 and cl 135.5 (not shown).

b) Synaptic vesicles preincubated with syntaxin and SNAP-25 in a stoichiometric ratio of 1:1 when incubated with the anti.ternary complex antibody showed that it bound to it as seen by the IgG bands in the Coomassie stained SDS-PAGE. Note that in the control where pre-incubation with syntaxin and SNAP-25 was omitted, no antibody bands were visible. 
e) Native gel showing that whereas cl 135.1 recognizes ternary complex, it cannot recognize a binary complex of syntaxin and SNAP-25.

To test the specificity of the antibodies for the complex, immunoblotting was done to determine whether only the complex and not the monomeric SNAREs is recognised by the antibodies. Soluble synaptobrevin, syntaxin and SNAP-25 were mixed in the stoichiometric ratio of 1:1:2 and pre-incubated for $1 \mathrm{~h}$ before SDS-PAGE/immunoblotting. As shown in Fig. 28a, the antibody (135.1) recognises only the complex and not the monomers, as asessed by the immunoblot and fluorescence signal on syntaxin. Similar results as in Fig. 28a were obtained for 135.2 and 135.5.

In some immunoblots using native material, a faint band of approximately $25 \mathrm{kDa}$ was visible (not shown). However, the antibodies do not recognise recombinant SNAP25. It is possible that during immunoblotting, native SNAP-25 acquires a conformation that enables its recognition by the antibodies.

To further test the binding of the antibodies to the complex, synaptic vesicles were pre-incubated with soluble syntaxin and SNAP-25. Antibodies were added to synaptic vesicles preincubated or not with syntaxin/SNAP-25. These mixes were pelleted down by high speed centrifugation $(100,000 \mathrm{x} \mathrm{g})$ and loaded onto SDS-PAGE followed by Coomassie staining. As shown in Fig. 28b, synaptic vesicles preincubated with syntaxin/SNAP-25 had higher signal for IgG bands of the antibody in the Coomassiestained gel. Whereas the ternary complex is resistant to SDS treatment, the binary complex between syntaxin and SNAP-25 is not. Therefore, native PAGE followed by immunoblotting for the antibodies confirmed that the antibodies recognise only the ternary complex and not the binary complex between syntaxin and SNAP-25 (Fig. 28c). 
Results

a

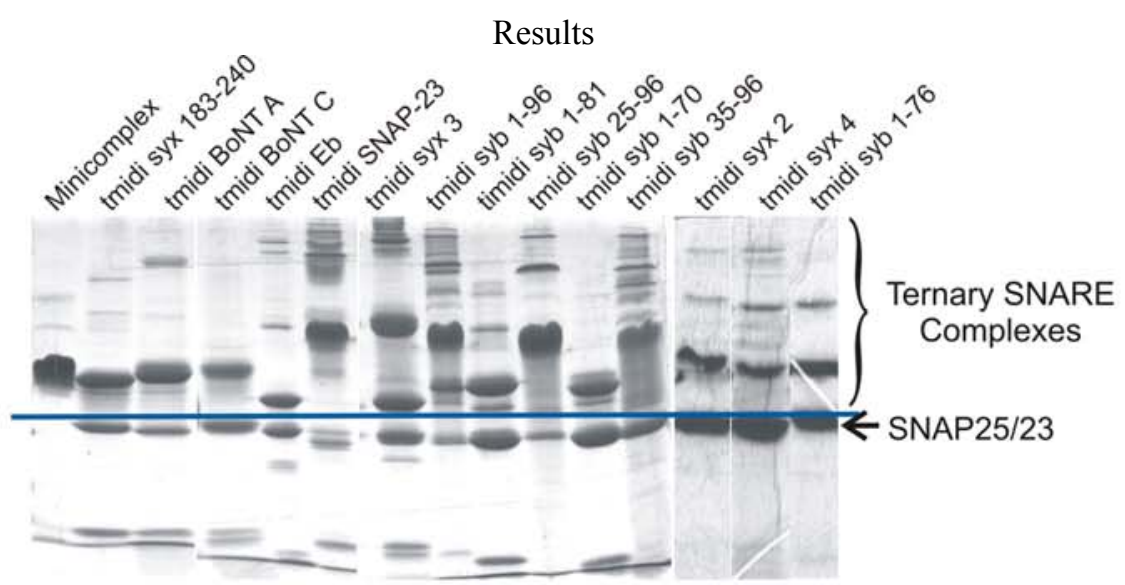

b
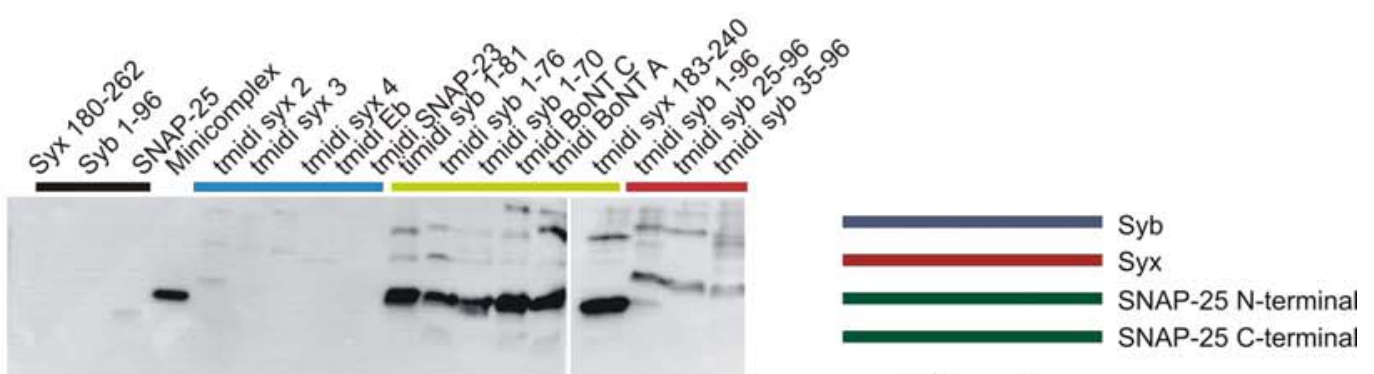

Minicomplex

cl 135.1

c


cl 135.2

d



represents monomers: syntaxin (syx), synaptobrevin (syb) and SNAP-25

represents replacement of a SNARE in tmidi with another SNARE:

(syx 2,3,4 replace syntaxin 1, endobrevin (Eb) replaces synaptobrevin, SNAP-23 replaces SNAP-25)

represents C-terminal truncations of a SNARE:

BoNT C and BoNT A indicates tmidi where SNAP-25 has aa 1-199 and 1-197 respectively

represents $\mathrm{N}$-terminal truncations of a SNARE 
Figure 29. Characterisation of the binding site of the novel monoclonal antibodies 135.1, 135.2 and 135.5 .

a) $10 \mu \mathrm{g}$ of all complexes tested were loaded on an SDS-PAGE followed by coomaise staining to determine whether they are SDS-resistant. All the bands above the blue line represent SDS-resistant SNARE complexes.

b), c) and d) $200 \mathrm{ng}$ of all proteins (monomers and complexes) were analysed by SDSPAGE followed by immunoblotting for the antibodies. tmidi: syx (aa 180-262), syb (aa 196) and SNAP-25, minicomplex: same as tmidi except that the SNAP-25 is without the linker between the two SNARE motifs (antigen).

b) $\mathrm{Cl} 135.1$ recognizes the N-terminal region of the SNARE complex more strongly. Cterminal deletions of the monomeric SNAREs does not affect its capacity to bind. Nterminal truncations of monomeric synaptobrevin compromises its capacity to bind the complex.

c) $\mathrm{Cl} 135.2$ recognizes the C-terminal region of the SNARE complex more strongly. Whereas N- terminal deletions of the monomeric SNAREs does not affect its capacity to bind, C-terminal truncations of monomeric synaptobrevin compromises its capacity to bind the complex. Note that C-terminal truncations of SNAP-25 does not affect its binding capacity.

d) Cl 135.5's binding preference is similar to that of 135.2 though the binding affinity is greatly reduced.

The next set of experiments were done to determine the binding site of the antibodies on the complex. Various complexes were generated: those with one of the SNAREs replaced with its isoforms and those that had truncations in either the C-terminal end or N-terminal end of one of the SNAREs. All complexes that were tested are resistant to SDS treatment (Fig. 29a). SDS-PAGE followed by immunoblotting with the individual antibodies revealed that whereas the $\mathrm{N}$-terminal end of the complex was crucial for the recognition of 135.1 (Fig. 29b), the C-terminal end of the complex, particularly the Cterminal end of synaptobrevin, appeared crucial for the recognition of 135.2 and 135.5 (Fig. $29 \mathrm{c}$ and d). The affinity of 135.5 for the complexes is the lowest.

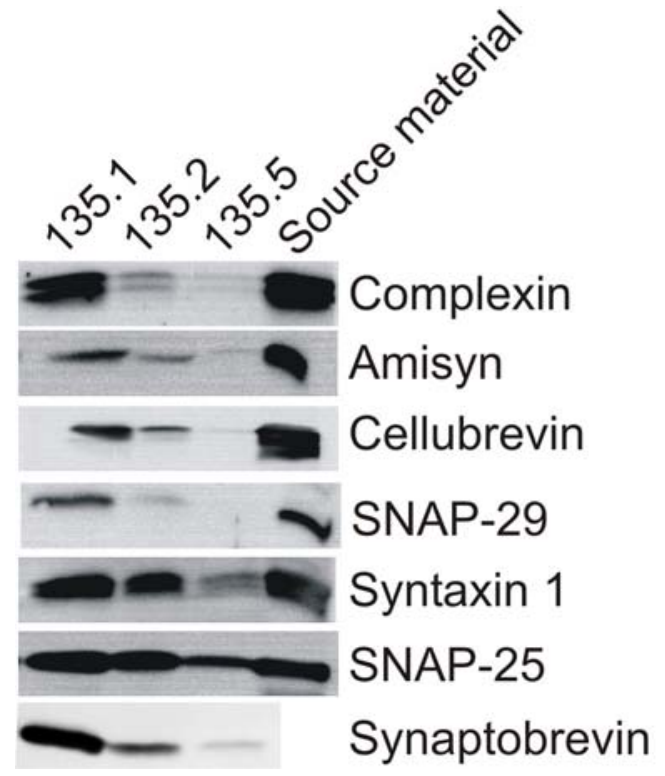

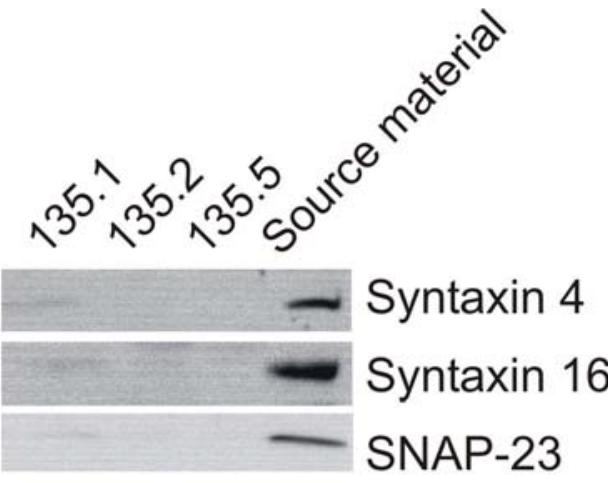

Negative controls 
Proteins identified by LC-coupled Electrospray Ionization (ESI) tandem mass spectrometry

\begin{tabular}{|c|c|}
\hline CHAPS extract & Triton $X-100$ extract \\
\hline syntaxin-1A [Rattus norvegicus] & syntaxin 1A [Rattus norvegicus] \\
\hline syntaxin 1B2 [Rattus norvegicus] & syntaxin 1B2 [Rattus norvegicus] \\
\hline SNAP-25b [Rattus norvegicus] & SNAP-25b [Rattus norvegicus] \\
\hline $\begin{array}{l}\text { vesicle associated membrane protein } 2 \text { [Mus } \\
\text { musculus] }\end{array}$ & $\begin{array}{l}\text { vesicle associated membrane protein } 2 \\
\text { [Mus musculus] }\end{array}$ \\
\hline $\begin{array}{l}\text { ATPase, } \mathrm{H}+\text { transporting, lysosomal V0 subunit a } \\
\text { isoform } 1 \text { [Mus musculus] }\end{array}$ & $\begin{array}{l}\text { ATP synthase gamma-subunit [Rattus } \\
\text { norvegicus] }\end{array}$ \\
\hline SNAP-25a [Rattus norvegicus] & tomosyn isoform $\mathrm{m}$ [Rattus norvegicus] \\
\hline VAMP-1 [Rattus norvegicus] & complexin 1 [Rattus norvegicus] \\
\hline synaptotagmin P65 - [Rattus norvegicus] & \\
\hline vacuolar-type $\mathrm{H}(+)$-ATPase [Homo sapiens] & \\
\hline $\begin{array}{l}\text { vacuolar adenosine triphosphatase subunit D [Mus } \\
\text { musculus] }\end{array}$ & \\
\hline
\end{tabular}

Figure 30. Immunoprecipitation followed by immunoblotting and mass-spectrometry of synaptopsome-enriched rat brain fraction.

The immunoprecipitates of 135.1, 135.2 and 135.5 were immunoblotted for various antibodies. The antibodies for the proteins indicated above produced positive results. Peptide generation by trypsinisation of the Triton X-100 and CHAPS extracted immunoprecipitates of cl 135.1 followed by Liquid Chromatography coupled Electrospray Ionization (ESI) tandem mass spectrometry analysis resulted in the detection of the proteins listed above.

To determine whether the antibodies specifically bind native neuronal complexes, immunoprecipitation of the complexes from a synaptosomal enriched fraction of the brain was done. In addition to syntaxin, synaptobrevin and SNAP-25, other SNAREs including amisyn, SNAP-29 and cellubrevin and a known binding partner of SNARE complex, complexin were detected in immunoblots (Fig. 30). Since the amount of proteins immunoprecipitated using 135.1 was the highest among the three antibodies tested, the extracts generated using two different detergents, CHAPS and TritonX-100, were used as starting material for immunoprecipitation with 135.1. Immmunoprecipitation was followed by SDS-PAGE and LC-coupled Electrospray Ionization (ESI) tandem mass spectrometry. The immunoprecipitates from the two different detergent extracts were used for mass-spectrometry because, owing to the difference in their micellar size $(6.15 \mathrm{kDa}$ for CHAPS and $80 \mathrm{kDa}$ for Triton X-100) (SigmaAldrich), there would be differential detection of SNARE complex interaction partners. In the immunoprecipitate of the Triton X-100 extract, in addition to the three SNARES (syntaxin 1, SNAP-25 and synaptobrevin), tomosyn, complexin 1 and ATP synthase gamma-subunit were detected (Fig. 30). In the immunoprecipitate of the CHAPS extract, in addtion to syntaxin, synaptobrevin and SNAP-25, synaptotagmin and vacuolar ATPase subunit D were detected (Fig. 30). 


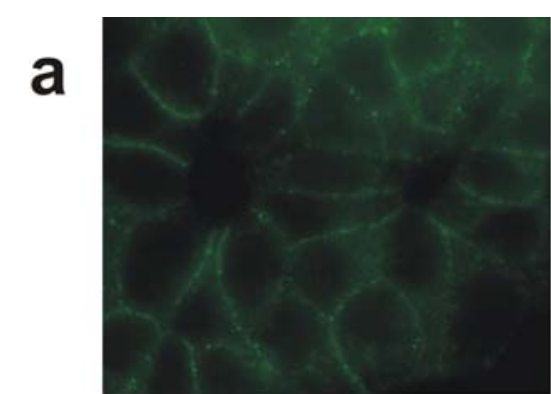

Ternary complex (135.1)



Ternary complex (135.1)

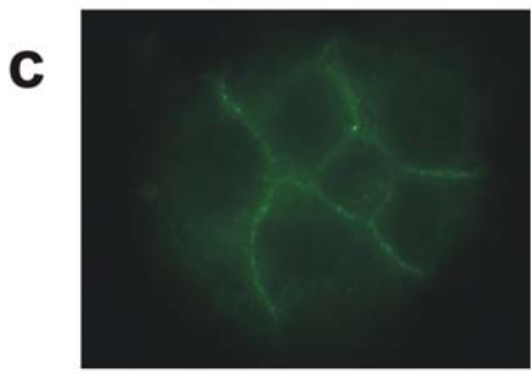

Ternary complex (135.1)

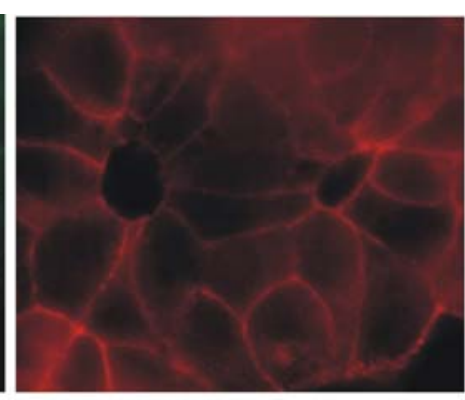

SNAP-25 (casanova)

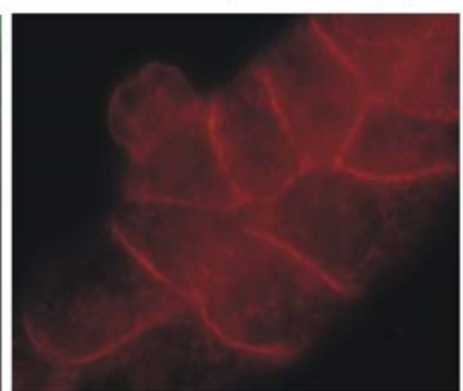

Syntaxin (R31)



Synaptophysin (G96)

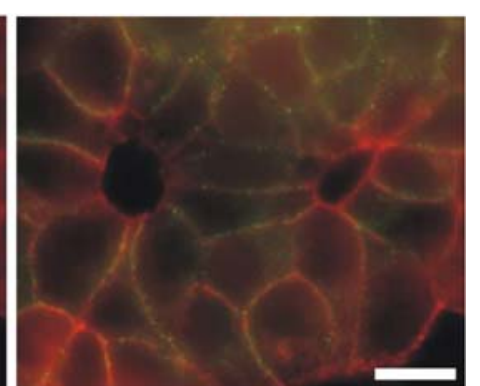

135.1/casanova overlay

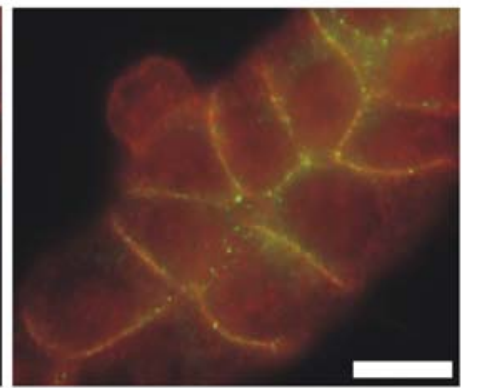

135.1/R31 overlay

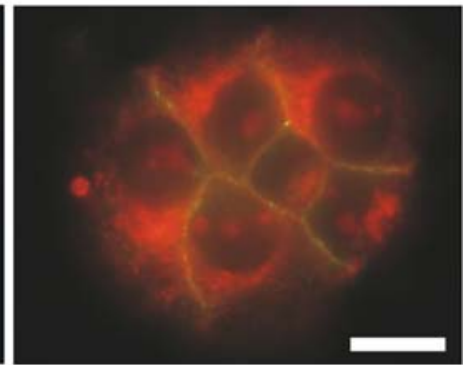

135.1/G96 overlay

Figure 31. Colocalization of ternary complex with membrane resident proteins, SNAP-25 (a) and syntaxin (b) and vesicular marker synaptophysin (c).

Note that like SNAP-25 and syntaxin, ternary complexes are mainly localized on the plasmamembranes. Also note that the vesicular marker synaptophysin is distributed throughout the cell

In immunostaining on PC-12 cells, 135.2 and 135.5 do not recognise specific signals (not shown) but 135.1 produced a punctate plasma membrane staining pattern (Fig. 31). To determine whether the recognition of the SNARE complex in a cell is specific, membrane patches of BHK cells and PC-12 cells were prepared as described in the Methods. In BHK cells, the neuronal SNAREs are absent. Thus the anti-neuronal SNARE complex antibody is expected not to bind to membrane sheets prepared from BHK cells. However, immunostaining of BHK cell membrane sheets resulted in some background staining (Fig.32, right panel) which was markedly reduced if the primary incubation and subsequent washing steps were done in the presence of $1 \%$ BSA and high 
salt $(500 \mathrm{mM})$ (Fig.32, middle panel), implying that though the antibody produces background staining, conditions can be controlled for minimising this effect.

The weak background staining observed in BHK cells, where neuronal SNAREs are normally absent suggests that the antibody's recognition of the antigen is specific, though the binding conditions need to be controlled, for instance by ensuring high salt concentration $(500 \mathrm{mM} \mathrm{NaCl})$ in buffers in the primary antibody incubation and subsequent washing steps.
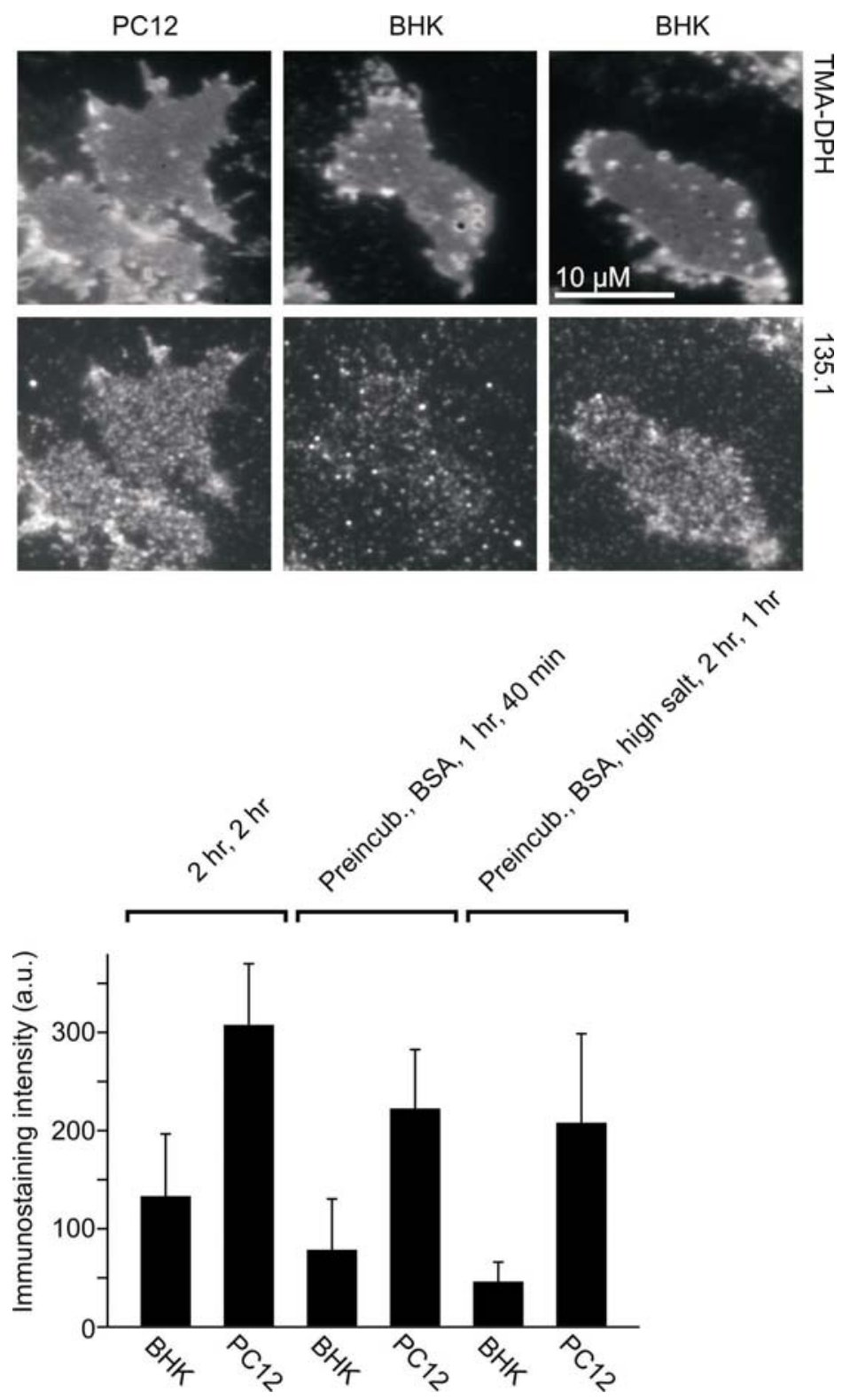

Figure 32. Supressing unspecific background of 135.1 staining on PFA-fixed membrane sheets.

Membrane sheets were produced from PC-12 cells and BHK cells (lacking neuronal ternary SNARE complexes), fixed and immunostained. $40 \%$ immunostaining intensity was observed on BHK membrane sheets when compared to that of PC-12 cells when incubation periods for both primary and secondary antibodies was $2 \mathrm{hr}$ and BSA as a blocker was not applied. Similar results were obtained when the incubation periods were shortened as indicated and BSA was applied as a 
blocker prior to antibody incubation. Relative BHK staining intensity could be strongly suppressed when the primary antibody and subsequent washing steps were done in the presence of $500 \mathrm{mM} \mathrm{NaCl}$ (see methods for details). Error bars are S.D. (These experiments were done in collaboration with T. Lang, MPI-bpc, Göttingen)

It was reported earlier that in PC-12 cells exogenously added synaptobrevin can engage plasma membrane syntaxin and SNAP-25 in a complex (Lang et al., 2002). The work in this thesis consolidates this finding. A measure of specific binding of antibody to the ternary SNARE complex was to determine to what extent incorporated fluorescencetagged synaptobrevin correlated with the antibody signal. A high degree of correlation was obtained (0.48+/- 0.6) (Fig. 33), strongly suggesting that the antibody's capacity to recognise SNARE complexes within a cell is high. However the low slope of the plot of incorporated fluorescent synaptobrevin versus anti-ternary complex antibody immunostaining signal suggests that the increasing signal of synaptobrevin fluorescence is disproportionate to that of the anti-ternary complex antibody. A possible explanation for this observation is that the anti-ternary complex antibody is occluded from its bonafide interaction sites by other factors or by the multimerisation of SNARE complexes, resulting a low signal to noise ratio. In contrast, synaptobrevin fluorescent intensity was proportionate to the immunostaining of an antibody that recognizes synaptobrevin (Fig. 33 ), indicating that its binding site is not sequestered by other factors. 
a
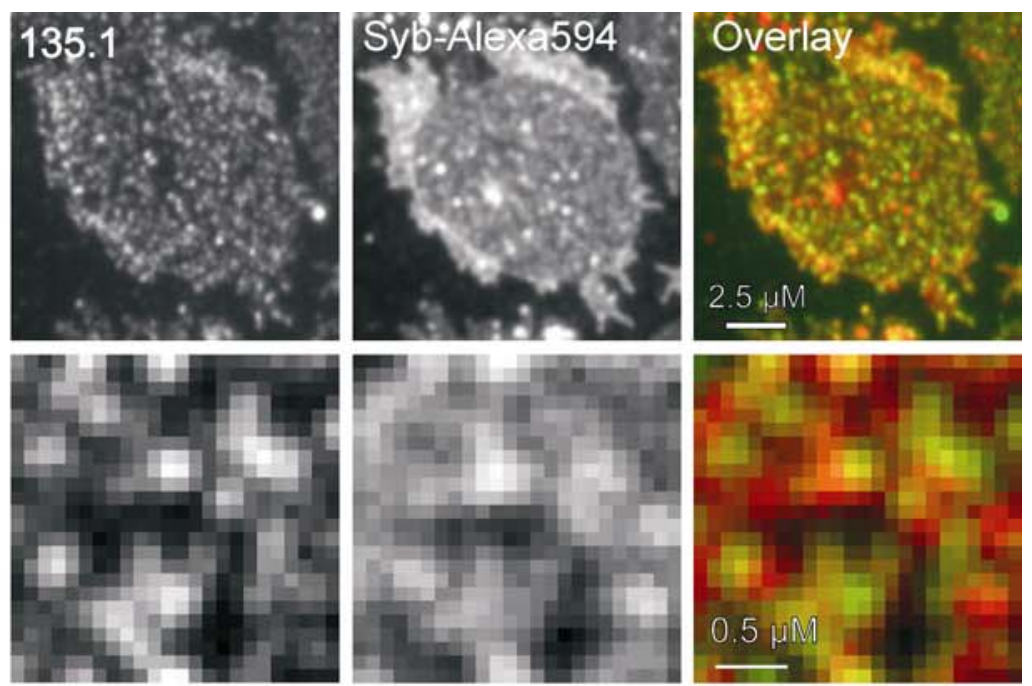

b
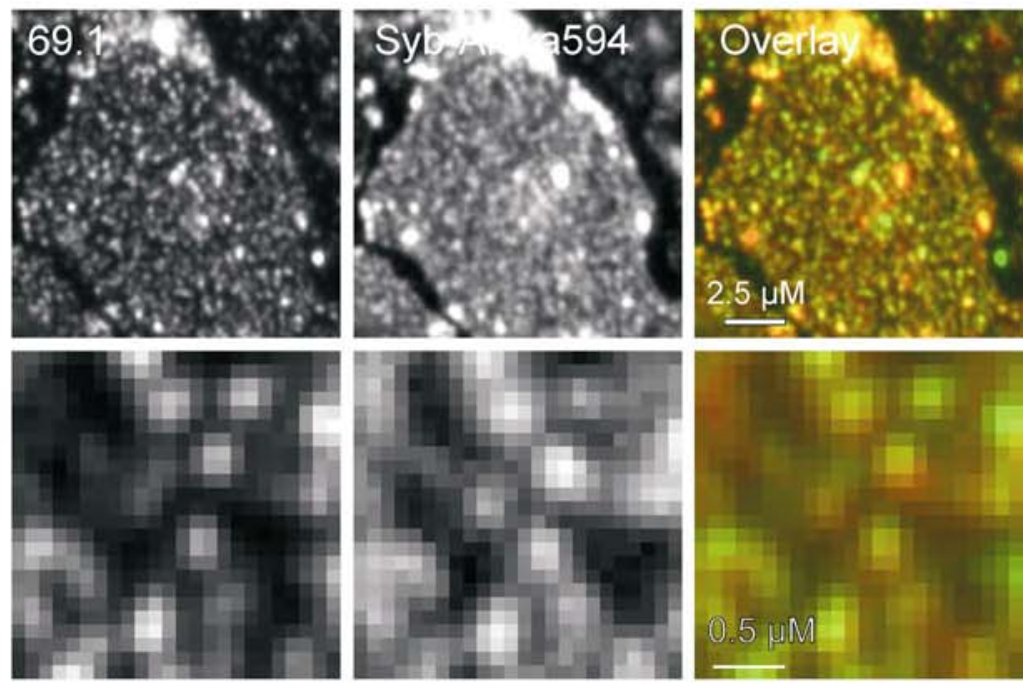

C

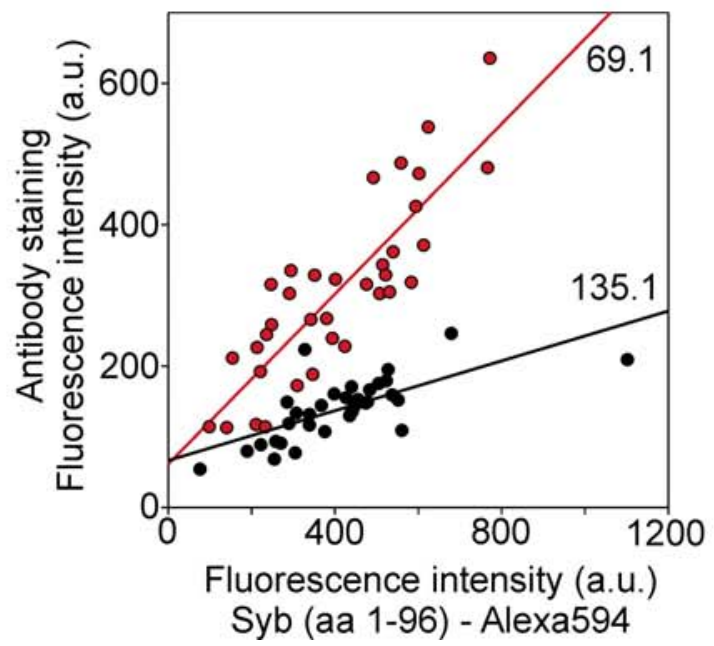


Figure 33. Correlation of signals arising from incorporated fluorescently labelled synaptobrevin with anti-ternary complex antibody 135.1 immunostaining (a) or with anti-synaptobrevin antibody 69.1 immunostaining (b).

( $\mathrm{a}$ and $\mathrm{b}$ ) Membrane sheets were produced from PC-12 cells. and then reacted with synaptobrevin 2-Alexa594 in order to form ternary SNARE complexes (red channel) followed by immunostaining with 135.1 (a) or 69.1 (b). Lower panels represent magnified views in a and b. The third panels in both a) and b) represent overlays from both channels. The correlation coefficient of signal of fluorescent synaptobrevin and 135.1 was $0.48+/-0.06(n=3$, on average 10 membrane sheets analysed in each experiment), that of fluorescent synaptobrevin and 69.1 was 0.72 (15 membrane sheets analysed).

c) Plotting green against red fluorescence intensity from individual membrane sheets from experiments shown in a and $b$. (These experiments were done in collaboration with Thorsten Lang, MPI-bpc, Göttingen)

\subsection{The anti-ternary complex antibodies block disassembly of SNARE complexes by NSF/ $\alpha$-SNAP}

Since the antibodies have been shown to bind to the neuronal SNARE complex and not to the monomers, it is expected that they should not have a marked effect on the assembly of SNARE complex but may impair the disassembly of the complex by NSF/ $\alpha$ SNAP. The assembly and disassembly of the SNARE complex was monitored by the FRET assay discussed above. Whereas an antibody against SNAP-25 (71.1) which is known to block assembly (Xu et al., 1999), does block donor fluorescence quenching of SNARE complexes in the FRET assay, none of the monoclonal antibodies against the complex blocks assembly either individually or all together (Fig. 34a). An antibody against synaptobrevin (69.1), which is known not to interfere with assembly (Lang et al., 2002), was used as a negative control (Fig. 34a). In the FRET assay developed for monitoring disassembly of pre-assembled SNARE complexes by NSF/ $\alpha$-SNAP, whereas all the three antibodies tested blocked disassembly to a certain degree, only 135.1 and 135.2 together blocked disassembly by an appreciable extent (Fig. 34b). These experiments suggest that the concerted block of disassembly of SNARE complex by 135.1 and 135.2, which probably bind to either end of the SNARE complex could be an important tool in functional assays and may even shed light on the disassembly mechanism. 
a

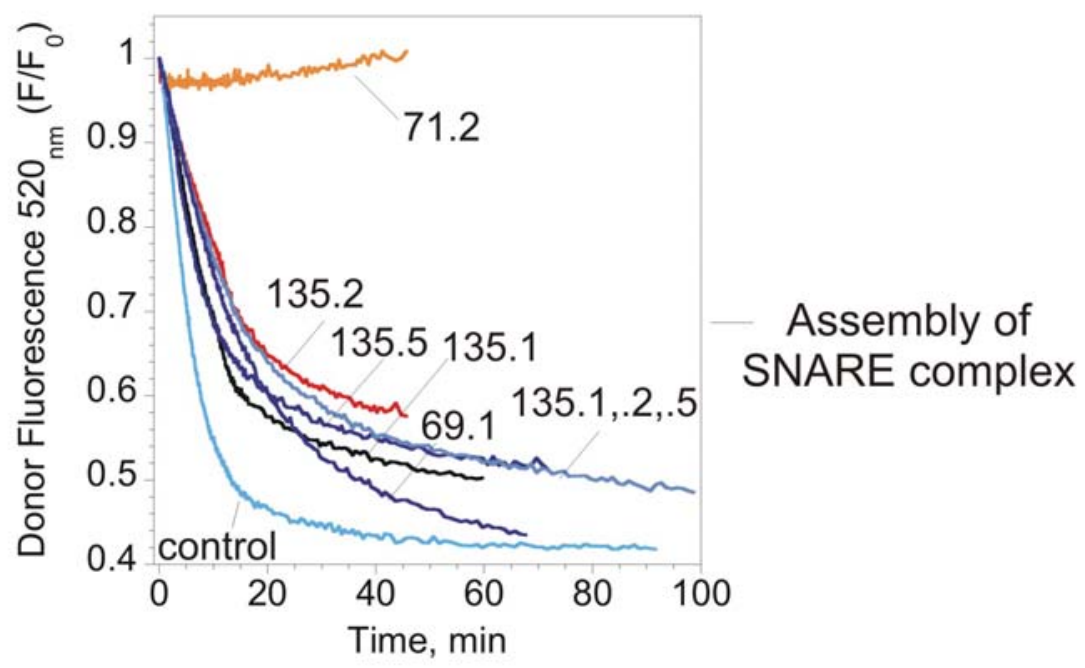

b

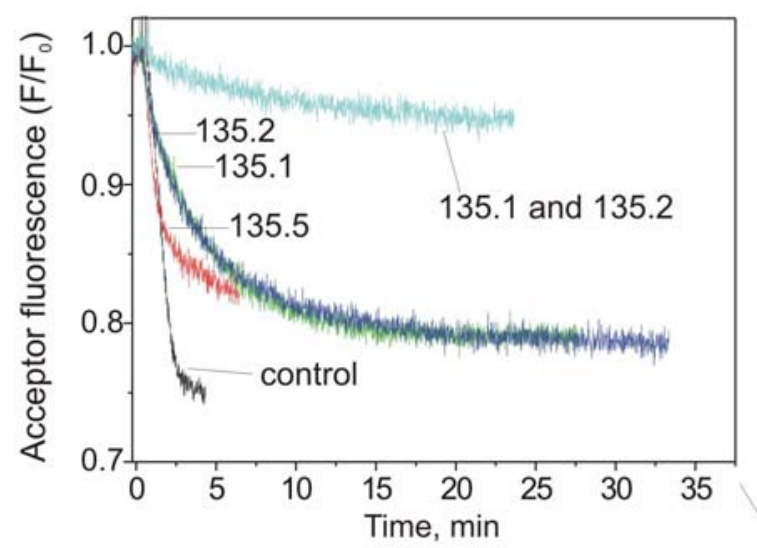

Disassembly of SNARE complex

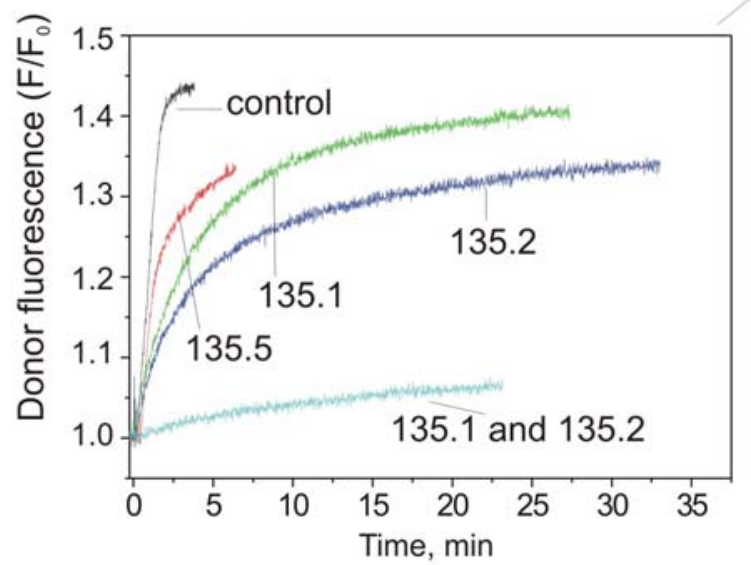

Figure 34. The anti-ternary complex antibodies can block disassembly of SNARE complexes by NSF/a-SNAP but does not abolish SNARE complex assembly, as assessed in a FRET assay.

a) Assembly of SNARE complex. Whereas purified monoclonal antibody against SNAP-25 (71.1, final conc. $32 \mathrm{ng} / \mu \mathrm{l}$ ), a known inhibitor of SNARE complex assembly (Xu et al., 1999) blocks donor fluorescence quenching, antibodies directed against synaptobrevin (69.1, final conc. $32.4 \mathrm{ng} / \mu 1$ ), known not to inhibit assembly (Lang et al., 2002) or against ternary SNARE complex (135.1 final conc. $10 \mathrm{ng} / \mu \mathrm{l}),(135.2$ final conc. $10 \mathrm{ng} / \mu \mathrm{l})$ and 
(135.5 final conc. $10 \mathrm{ng} / \mu \mathrm{l}$ ) alone or all together could not block assembly. (see legend to Fig. 11a)

b) Disassembly of SNARE complex (80 nM final conc. of synaptobrevin ${ }^{280 \mathrm{G}}$ (aa 1-96), syntaxin aa $180-262$, SNAP-25 $5^{130 T R}$ full-length) by NSF $(8 \mathrm{nM}) / \alpha$-SNAP $(1.6 \mu \mathrm{M})$. Although, each of the antibodies 135.1, 135.2 and 135.5 (0.5 $\mathrm{ng} / \mu \mathrm{l}$ final concentration) blocks SNARE complex disassembly to a certain level, appreciable block was possible only when both 135.1 and 135.2 were used in concert. All disassembly reactions were carried out in Hepes-NaOH, pH 7.4, 2mM ATP, $5 \mathrm{mM} \mathrm{MgCl}_{2}, 0.5 \%$ CHAPS. (Figure b was contributed by Ulrike Winter, MPI-bpc, Göttingen)

\subsection{The anti-ternary complex antibodies do not prevent release from permeabilized PC-12 cells}

To test whether the antibodies can inhibit release in a functional assay, the permeabilized cell assay (Hay and Martin, 1992; Klenchin et al., 1998) was used. Permeabilized PC-12 cells have been extensively studied to monitor release under different conditions. This assay was adapted to test whether PC-12 cells perfused with anti-ternary complex antibodies blocked release of secretory granules. Perforations were generated in the PC-12 cells that had been pulsed with $[3 \mathrm{H}]$ labelled Norepinephrine by treatment with Streptolysin-O (Ahnert-Hilger et al., 1993). The perforated cells, pretreated with a mixture of all the three antibodies were stimulated for 25 minutes. The antibodies were accessible into the permeabilised cells (Fig. 35b). As shown in Fig. 35a, the antibodies had no impact on release, suggesting that the releasable pool of vesicles had either pre-formed SNARE complexes or SNARE complexes formed upon stimulaton. The binding of antibodies to the complexes did not prevent them from fusing membranes. Since this assay runs down in a short duration, a second round of release - which is be expected to be retarded by the antibodies-cannot be monitored. 
a

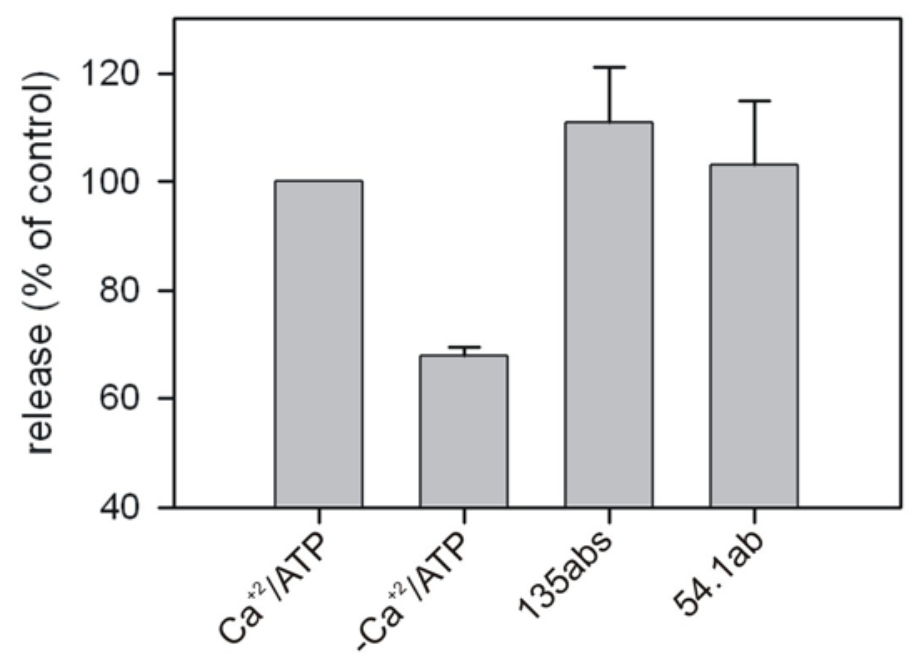

b

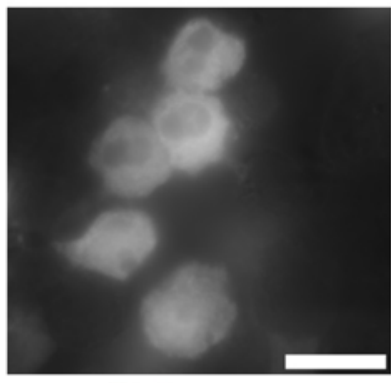

+ SLO



$-S L O$

Figure 35. $[3 \mathrm{H}]$ Norepinephrine secretion from Streptolysin-O permeabilized PC-12 cells.

a) Permeabilized cells were incubated with anti-ternary complex antibodies (135.1, 135.2, 135.5) or anti-NMDA receptor antibody 54.1 for $12 \mathrm{~min}$ prior to stimulation. Then calcium and ATP were added and release was measured after $25 \mathrm{~min}$ of incubation. None of the antibodies inhibited release. Graph shows mean \pm SEM (four expts.)

b) The antibodies have access into pearmeabilised cells. SLO-permeabilized (left) and nonpermeabilized (right) cells were incubated with 135 antiobodies as in the release assay followed by detection with a Cy3 conjugated secondary antiobody.

(Figure b was contributed by Silvio Rizzoli, MPI-bpc, Göttingen) 


\subsection{Preliminary results suggest that the total amount SNARE complex in a resting cell and stimulated cell is comparable}

Does the amount of SNARE complex in a cell increase when it has been stimulated or does the cell's disassembly machinery - NSF/ $\alpha$-SNAP - act immediately after exocytosis has occurred to maintain the concentration of SNARE complex in a cell? Previous reports have indicated that under resting conditions, the amount of SNARE complex can be significantly increased (Lonart and Sudhof, 2000). To determine whether SNARE complexes accumulate in synaptosomes that have been stimulated, an assay that can measure SNARE complexes directly is most suited. The novel antibodies discussed in this thesis provide an excellent tool to measure SNARE complexes in native membranes. Synaptosomes prepared from rat brains were stimulated by the application of either $\mathrm{KCl}$ or ionomycin, a $\mathrm{Ca}^{+2}$ ionophore. To test whether the synaptosomes treated with $\mathrm{KCl}$ or ionomycin had indeed been stimulated, the synaptosomes were subjected to osmotic lysis and separated into a plasma membrane rich (LP1) and a vesicle enriched fraction (LP2) followed by SDS-PAGE and immunoblotting for Rab3a, a small GTPase known to dissociate from vesicles upon stimulation (Fischer von Mollard et al., 1991). As shown in Fig. 36, in $50 \mathrm{mM} \mathrm{KCl}$ or $10 \mu \mathrm{M}$ ionomycin treated synaptosomes, the amount of Rab3a reduced by almost $60 \%$ in the vesicular fraction whereas it remained unchanged in unstimulated synaptosomes. In both stimulated and unstimulated synaptosomes, comparable amount of SNARE complexes were present (Fig. 36 c), suggesting that they disassemble immediately after exocytosis has occurred. 
a

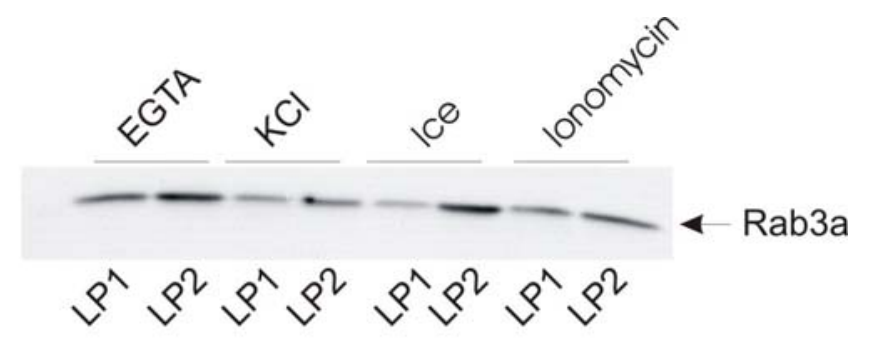

b

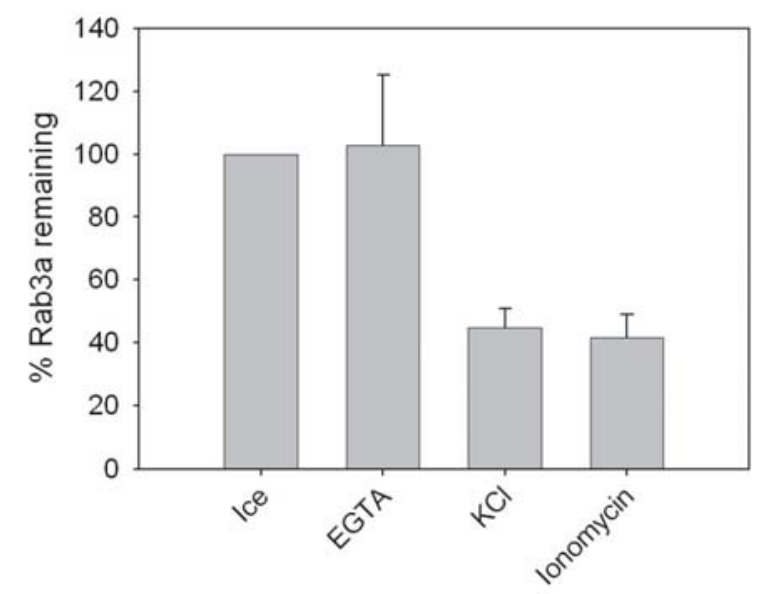

C

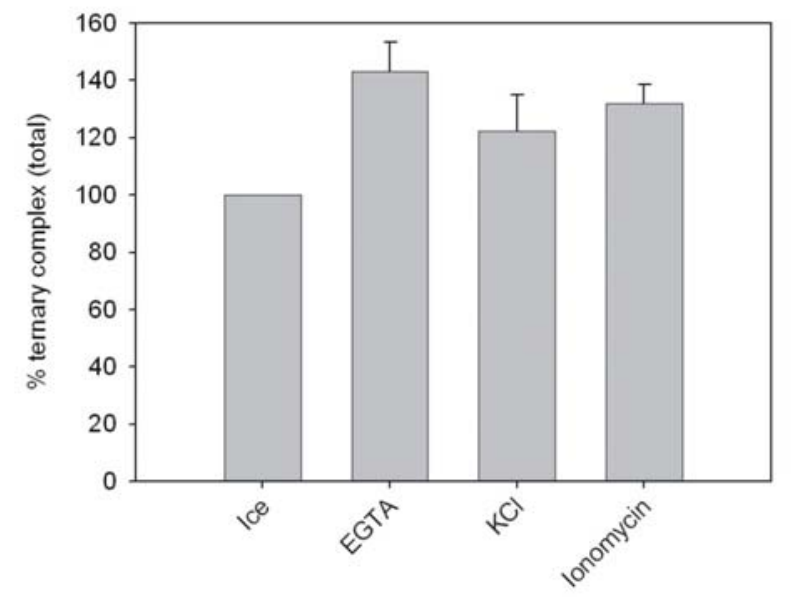

Figure 36. Are ternary complexes dissociated before or after synaptic vesicle endocytosis?

Synaptosomes were generated from rat brain. They were stimulated by the addition of high potassium or ionomycin. To monitor ternary complexes, synaptosomes were loaded directly onto the gel. To assess whether synaptosomes had indeed been stimulated, they were ruptured via hyperosmotic shock. Large membranes were pelleted by differential centrifugation (LP1). Synaptic vesicles and newly endocytosed vesicles were then pelleted by prolonged high speed centrifugation $(33,000 \mathrm{~g}$, LP2).

a) Typical immunoblots of stimulated and unstimulated synaptosomes, ice (synaptosomes kept on ice), EGTA (synaptosomes incubated at $37^{\circ} \mathrm{C}$ in the presence of EGTA), $\mathrm{KCl}$ (synaptosomes incubated with $50 \mathrm{mM} \mathrm{KCl}$ in the presence of $\mathrm{Ca}^{+2}$ at $37^{\circ} \mathrm{C}$ ), ionomycin (synaptosomes stimulated with $10 \mu \mathrm{M}$ ionomycin in the presence of $\mathrm{Ca}^{+2}$ at $37^{\circ} \mathrm{C}$ ). Immunoblot of anti-Rab3a antibody 42.1 . 
b) Efficiency of stimulation. Rab3a dissociates from synaptic vesicles during the fusion process (Fischer von Mollard et al., 1991). Note the loss of Rab3a in ionomycin or KCl conditions. Graph shows mean \pm SEM, four experiments. Densitometry was performed on LP2 fractions.

c) Ternary complex does not increase in the stimulated synaptosomes. Synaptosomes were stimulated as above and the amount of ternary complex was quantified (mean $\pm \mathrm{SEM}$, four experiments). Note that the total amount of complex remains unchanged despite massive exocytosis.

\subsection{SNARE complexes are present on synaptic vesicles}

That vesicle endocytosis occurs even before SNARE complexes are disassembled on the plasma-membrane is a distinct possibility. Immunoblotting of subcellular fractions of rat brain revealed substantial amount of SNARE complexes in the vesicular fraction (Fig. 37d). Furthermore, direct imaging of isolated vesicles revealed that a majority of synaptic vesicles (about 80\%) have ternary complexes on them (Fig. 37a and c). The intensity of the signals due to anti-ternary complex antibodies on these vesicles was markedly lower than that of vesicle resident proteins like synaptophysin, indicating that very few copies of ternary complexes were present on the vesicles. It cannot be ruled out though that SNARE complexes form on isolated vesicles when they are not subjected to disassembly by NSF/ $\alpha-S N A P$ 


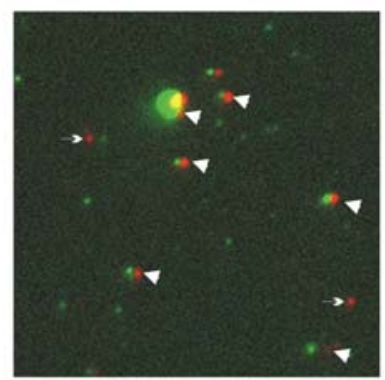

C

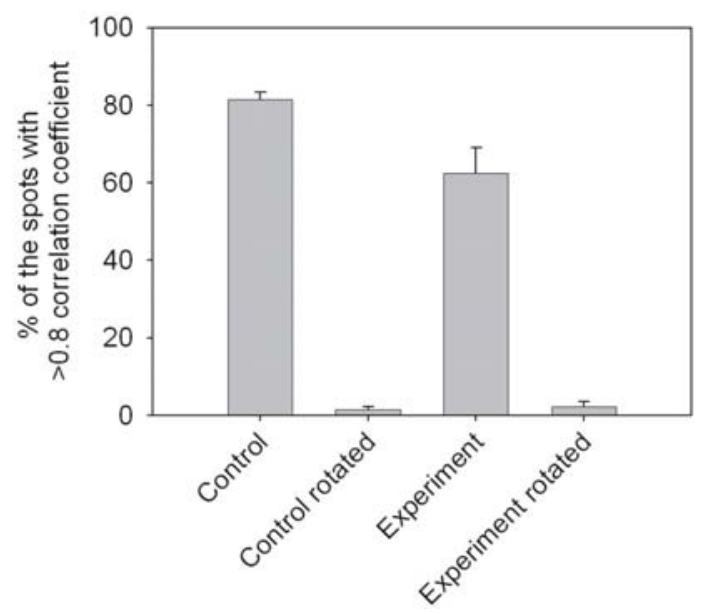

Results

b

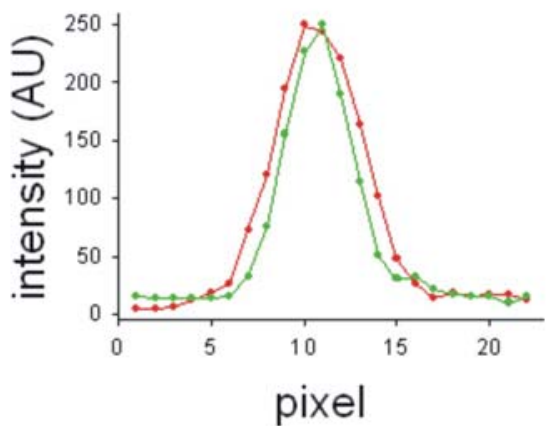

d

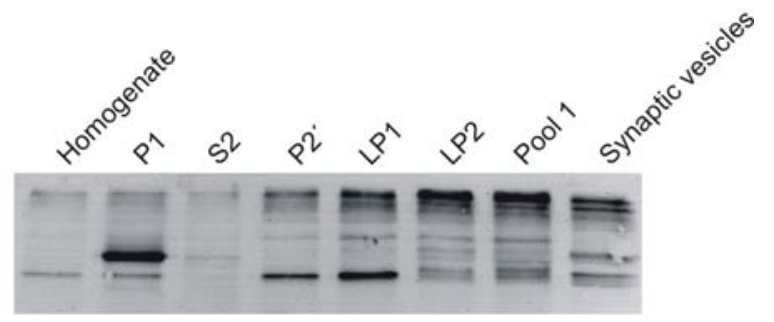

Figure 37. A large fraction of synaptic vesicles binds anti-ternary complex antibodies.

Synaptosomes were prepared according to (Nicholls and Sihra, 1986), and a synaptic vesicleenriched fraction was prepared (LS1). The vesicles were labeled in suspension with 135.1 antibodies, centrifuged onto glass coverslips, further labeled with G96 rabbit anti-synaptophysin antibodies, and finally stained with fluorescently labeled goat anti-mouse and anti-rabbit antibodies.

a) Images were acquired in the green (135.1) and red (G96) channels, the two frames were aligned using Tetraspeck beads, and then shifted by 5 pixels in the $\mathrm{X}$ axis, to indicate the colocalizing green and red spots (marked by arrows). Some vesicles (identified by the bright G96 staining) do not exhibit 135.1 staining (arrowheads).

b) 24-pixel long line scans were drawn through the centers of randomly selected G96positive organelles; the intensities in the green and red channels along the scans were obtained and plotted as in the example above. The correlation between the two fluorescence distributions was then measured (see Methods).

c) c) The percentage of highly correlating spots (correlation coefficient higher than 0.8) was obtained for the experimental condition. Positive control represents synaptic vesicles labeled for synaptophysin and stained with two differently colored secondary antibodies; negative controls represent the correlation between images obtained in the red channel and the mirror images of the green channel. $\mathrm{N}=3$ independent experiments for each condition (means +/- SEM).

d) Subcellular distribution of Ternary SNARE complex. Brain subcellular fractions (10 $\mu \mathrm{g} /$ lane) were analyzed by SDS-PAGE followed by immunoblotting for anti-ternary complex antibody 135.1 .P1, low speed (nuclear) pellet P2', enriched synaptosomal fraction; $L P 2$, enriched synaptic vesicles; $L P 1$, membrane pellet obtained after hypotonic lysis of P2'; P1, low speed (nuclear) pellet; pool 1, large organeller fraction. For details of subcellular fractionation, see (Hell, 1994; Huttner et al., 1983; Nagy et al., 1976) 


\section{$4 \quad$ Discussion}

Genetic, physiological and biochemical studies have established the role of SNARE proteins at a late stage of exocytosis (references in Introduction). SNARE complex formation is accompanied by a large enthalpy of binding of unstructured monomeric SNAREs (Fasshauer et al., 2002). This energy can be transduced via the linker regions of SNAREs to the membranes, perturbing them thereby and inducing fusion (Knecht and Grubmuller, 2003).

In vitro studies using soluble domains of SNAREs have shown that SNARE complex formation is spontaneous and irreversible (Fasshauer et al., 1997b). The complex thus formed is kinetically stable and there appears to be no additional input of energy in the fusion reaction. The free energy of assembly cannot be measured due to a marked hysteresis, i.e., SNARE complex assembly and disassembly pathways are divergent (Fasshauer et al., 2002). The kinetic stability of the SNARE complex is ideally suited to ensure that trans-complex formation, once initiated, is brought to completion. The monomeric SNAREs are regenerated for another round of fusion by the AAA+ ATPase NSF and its co-factor $\alpha$-SNAP (Sollner et al., 1993a; Sollner et al., 1993b).

Synaptobrevin 2 is the R-SNARE localized to synaptic vesicles. Mice that are deficient in synaptobrevin have severely impaired evoked neurotransmitter release (Schoch et al., 2001). Synaptobrevin is also the target for most of the clostridial neurotoxins that target the SNAREs, Botulinum toxins B, D, F, G and Tetanus toxin, underscoring the importance of this protein in neuronal physiology, reviewed in (Breidenbach and Brunger, 2005). Synaptobrevin comprises about 10\% of all proteins (or 60-70 copies) on a single synaptic vesicle (Takamori unpublished observations). The regulation of such an abundant and reactive molecule is therefore of paramount significance.

Studies on double knock-out mice lacking synaptobrevin and another R-SNARE, cellubrevin have revealed that the non-conserved N-terminal domain of synaptobrevin (residues 1-32) regulates the priming activity of synaptobrevin (Borisovska et al., 2005). The N-terminal domain of synaptobrevin is proline rich and is therefore a potential target for regulatory molecules though no such factor is known. The determination of such a regulatory factor may not have been possible due to the transient nature of binding. Thus, 
as suggested for SNAP-25 (Hess et al., 1992; Koticha et al., 2002; Lane and Liu, 1997; Nagy et al., 2002; Nagy et al., 2004) and syntaxin (Betz et al., 1997; Dulubova et al., 1999; Sassa et al., 1999), the regulatory domain on synaptobrevin may not be solely encoded in the SNARE motif. The specificity of pairing of its SNARE domain, the interactions of its membrane-proximal region with the membrane and/or other regulatory factors and the dynamics of its trans-membrane region may confer additional regulatory features on synaptobrevin.

SNAREs may be regulated by the specificity of SNARE pairing. Soluble RSNAREs, tomosyn and amisyn, bind syntaxin and SNAP-25 resulting in fusionincompetent complexes (see Introduction). Synaptobrevin cannot replace tomosyn (Pobbati et al., 2004) or amisyn (Pobbati, PhD thesis) to form the fusogenic complex with syntaxin and SNAP-25 ensuring that the number of fusion competent acceptor complexes between syntaxin and SNAP-25 is tightly controlled. The existence of non-cognate SNARE complexes could thus be a means of fine-tuning SNARE regulation.

Liposome fusion experiments carried out by Rothman and colleagues led to the proposal that the specificity of fusion is determined by the SNAREs (McNew et al., 2000; Parlati et al., 2000). Another study has, however, shown that both cognate and noncognate SNAREs engage to form complexes (Fasshauer et al., 1999). Moreover, several SNAREs may be operating in more than one pathway, for instance, vesicles bearing Snc1 or Snc2 can fuse with plasma membrane, trans-golgi network and early endosomes (McNew et al., 2000; Paumet et al., 2004). In synaptobrevin knock-out mice, cellubrevin expression is up-regulated by $36 \%$ (Borisovska et al., 2005). Likewise, in cellubrevin knock-out mice, the expression of synaptobrevin is up-regulated by $27 \%$ (Borisovska et al., 2005). These results, among other similar studies in yeast and Drosophila (Bhattacharya et al., 2002; Liu and Barlowe, 2002; Vilinsky et al., 2002) suggest that SNAREs can compensate the deficiency of their isoforms to a certain extent. It is therefore possible that there is a degree of functional redundancy among SNARE proteins.

The evolutionary design of the synaptic SNAREs is best suited to orchestrate neuronal exocytosis with precise kinetics. Each of the synaptic SNAREs has structural features that subject it to regulation. In this study, the activity of synaptobrevin in membranes was studied. In the second part of this study, an antibody raised against the neuronal SNARE complex was characterised and the status of the SNARE complex in a cell was investigated. 


\subsection{Synaptobrevin in membranes is fully active}

It has been suggested that synaptobrevin is under direct regulation by the membrane (Kweon et al., 2003b). In a separate study, synaptobrevin in synaptic vesicles was suggested to be inhibited from interaction with syntaxin and SNAP-25 (Hu et al., 2002). Together, these findings suggest that synaptobrevin in membranes is intrinsically inactive and that it has to be activated by the calcium sensor, synaptotagmin, prior to SNARE complex assembly.

In the present study, exogenously added SNAP-25 and syntaxin were capable of driving surface-exposed synaptobrevin (as measured by its susceptibility to the protease Tetanus toxin) completely into SNARE complexes.

The differences in the results in my study and that of Kweon et al. are noteworthy. Shin and colleagues found no measurable binding of synaptobrevin to partner SNAREs, as assessed by electron paramagnetic resonance studies (Kweon et al., 2003b). Though the phospholipid composition and protein : lipid ratio was somewhat different to that used in my study, I observed SNARE complex assembly to a measureable degree in liposomes made of different lipid compositions. Since the reconstitution procedure used by Shin and colleagues was different, using insertion of protein into preformed liposomes instead of simultaneous reconstitution from micellar solutions, in a pilot experiment using a protocol similar to that of Kweon et al, I again found no evidence for inactivation of synaptobrevin (not shown).

The results also suggested that 1) synaptobrevin activity on membranes is an intrinsic property of SNAREs, i.e, given the correct physical environment, synaptobrevin in membranes spontaneously engages syntaxin and SNAP-25 in a complex and 2) like other protein-protein interactions, synaptobrevin binding to its partner SNAREs is stabilised by weak counter-ions.

An interesting observation was that synaptobrevin became refractory to SNARE complex formation after prolonged storage or after frequent freeze-thaw cycles. Synaptobrevin on these liposomes could not be cleaved by Tetanus toxin light chain. Investigation by electron microscopy (not shown) revealed that aged and frequently frozen-thawed liposomes tended to clump together and form larger clusters, thus preventing synaptobrevin from interacting with other proteins. 
I was also unable to reproduce the findings of Davletov and colleagues who reported that membrane-anchored synaptobrevin, either reconstituted in liposomes or in purified synaptic vesicles was unable to form complexes with exogenous syntaxin and SNAP-25 unless detergent was added (Hu et al., 2002). Though we have no obvious explanation for the discrepancy between our results and that of Davletov and colleagues, it is conceivable that the differences in protein purification and vesicle enrichment protocols may account for some of the differences. It is noteworthy that $\mathrm{Hu}$ et al. extracted the proteins from preparative SDS-PAGE. Since SDS binds to hydrophobic sufaces very tightly, it may not be possible to unbind SDS by simple dialysis. SDS bound to SNARE proteins would interefere with SNARE complex assembly.

Several laboratories reported that synaptobrevin-containing liposomes readily fuse with liposomes containing SNAP-25 and syntaxin, a reaction that clearly requires active synaptobrevin (Schuette et al., 2004; Tucker et al., 2004; Weber et al., 1998). In the liposome fusion assays used by researchers as a way of mimicking intra-cellular membrane fusion events, however, it has not been clear how active the SNAREs are. In the present study, the reactivity of synaptobrevin in synaptic vesicles and lipsomes were shown to be comparable implying that the propensity of synaptobrevin in membranes to engage partner SNAREs, syntaxin and SNAP-25 in a complex is its intrinsic property, uninfluenced by other proteins, as suggested by previous studies (Chen et al., 2004; Hu et al., 2002; Hu et al., 2003; Kweon et al., 2003b; Quetglas et al., 2000).

The proposed inaccessibility of synaptobrevin for complex formation also does not explain the action of clostridial neurotoxins that readily cleave membrane-bound synaptobrevin both in liposomes and in synaptic vesicles. In order to cleave synaptobrevin, clostridial neurotoxins require access to most of the cytoplasmic domain of synaptobrevin, for instance the cocrystal structure of synaptobrevin bound to botulinum neurotoxin type B at $2.0 \AA$ resolution showed that BoNT B has extensive interactions with residues N-terminal to the cleavage site 76-77 (Hanson and Stevens, 2000). The present study provides a convenient explanation for these findings by showing that the cytoplasmic portion of membrane-bound synaptobrevin is free to interact with other proteins. 


\subsection{Role of membrane-proximal tryptophan residues of synaptobrevin}

The reason for the inaccessibility of synaptobrevin to soluble cognate SNAREs was assigned to the membrane-dipping of the membrane-proximal residues (aa 85-92). These residues were suggested to form an amphipathic helix dipping into the membrane at an angle of $33^{\circ}$ mediated by interfacial tryptophan residues (Kweon et al., 2003b). The studies by Shin and colleagues who reported that insertion of the membrane-proximal region of synaptobrevin into the membrane is responsible for its inactivation implies that if tryptophan residues are replaced by smaller, polar amino acids, the block by the membrane would be relieved and SNARE complex formation would be markedly accelerated. In the present study though, I was unable to detect any difference in SNARE complex formation rate between wild type synaptobrevin and a mutant in which two conserved membrane-proximal tryptophan residues were replaced by serines.

Surprisingly, no such inactivation was observed by Shin and colleagues for the yeast synaptobrevin homolog Snc2p, and spin-labeling of the membrane proximal region of Snc2p suggested that this region is not membrane-inserted (Chen et al., 2004). Since Snc2p is involved in constitutive secretion, it was proposed that the structural difference between Snc2p and synaptobrevin membrane-proximal regions represents the difference between constitutive and regulated secretion (Chen et al., 2004). This hypothesis proposes that whereas R-SNAREs involved in constitutive secretion would be constitutively active, R-SNAREs involved in regulated exocytosis would require an activation step. This activation was proposed to be carried out by the calcium sensor, synaptotagmin (Hu et al., 2002).

The membrane-proximal tryptophan residues suggested to be critical for synaptobrevin inactivation are conserved between synaptobrevin and Snc2p. It is intriguing that such divergent roles would arise for very highly conserved residues, as illustrated in the sequence alignment below, in proteins belonging to the same sub-family. It is not rare for tryptophan residues to be found near the membrane-water interface in many single-spanning membrane proteins. In such proteins, they are part of characteristic belts containing both basic and aromatic residues near the membrane-water interface (Killian and von Heijne, 2000). The indole side chain of tryptophan is ideally suited for interacting with polar-apolar membrane-water interface. Whereas the large hydrophobic aromatic ring is preferentially buried in the hydrophobic part of the bilayer, the amide 
group attached to this aromatic ring can interact with the polar environment of the interface. Such a property can also be suggested for tyrosine. Not surprisingly, the membrane-proximal residue of synaptobrevin (aa 88) is tyrosine. In addition, the positively charged amino acids Lys and Arg might be expected to have a special interaction with the interface. Whereas the positively charged residues can 'snorkel' into the phospholipids head groups, the polar-aromatic residues penetrate into a region near the lipid carbonyls (Killian and von Heijne, 2000).

MuMre_Syb4/1-141 AsPa_snc/1-122 AsNig_Snc/1-119 ScPo_Snc/1-121 SaCe_Snc $2 / 1-115$ KIL_SSC/1-110 HyJe_Sno/1-111 $\mathrm{NeCr} \mathrm{SnC}_{-11-119}$ Gize_Sno/1-118 HMe_syb 1/1-169 LySt_syb 1/1-145 ScJa_syb 3*/1-160 CaBr_snb 1/1-108 CaEI_Snb 1/1-109 StPu_syb 1b/1-104 GaGa_Syb 2/1-114 DaRe_syb 2/1-110 RaNo_Syb 2/1-116 MuMu_syb 2/1-116 RaNo_Syb 2b/1-13t HoSa_syb 2/1-116 MaMu_syb 2/1-116 OrCu_syb 2/1-81 XeLa_syb 1a/1-114 XeTr_syb 1/1-114 DaRe_syb 1/1-119 RaNo_syb 1/1-117 HoSa_syb 1-1/1-111 HoSa_syb 1-3/1-11t XeLa_sybPMN1-10: DrPs_Syb/1-135 DrMe_Syb_a/1-132

Conservation

Quality

Consensus

\section{Membrane-Proximal Tryptophan Residues}

\author{
$100 \quad, \quad 110$ \\ $10 \quad 120$
}

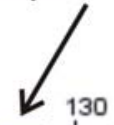

140

LDELLDKS'ESLSD DNATAF'SNRS'KQLRRQ'MMURG'CKIKÁ IMALÁAAIL LDSLQDKTDNLAVSAQGFRRGANRVRKQMWWKDMKMRVCLVICVILL LDSLQDKTDNLATSAQGFRRGANRVRKQMWWKDMKMRVCL IVCIIIL LDSLQDKTDNLAVSAQGFRRGANRVRKKMWWKDMRMRLC। I G I I L LTS I EDKADNLA I SAQGF KRGANRVRKQMWWKDLKMRMCLFLVV I IL LTSIQDKADNLAVSAQGF KRGANRVRKQMWWKDLKMRMCLVLVVI IL LDVLQDKTDNLAESAQGFRRGANRVRKQMWWKDMKMRVCIVVG। ILL I DALQTKTDDLA I SAQGFRRGANQVRKKMWWKDMKMR I CLIVG I I L LDALQDKTDNLAVSAQGFRRGANRVRKQMWWKDMKMRMCLI IG I I L LAELDGRADALQAGASQFEASAGKLKRKFWWKNMKMML IMGAVVAVV I SQLDDRAEALQAGASQFEASAGKLKRKYWWKNCKMML ILGA I IGI I LSQLDDRADALQAGASQFEASAGKLKNKYWWKNMKMNI I IGGVALVL LSQLDDRADALQEGASQFEKSAATLKRKYWWKN I KMMI IMCA I VVIL LSQLDDRADALQEGASQFEKSAATLKRKYWWKN I KMMI I MCA I VVIL LSVLDDRADALQQGASQFETNAGKLKRKYWWKNCKMMI ILA I I I IV I LSELDNRADALQAGASQFETSAAKLKRKYWWKNCKMMI I LGVVCTVI LSELDDRADALQAGASQFETSAAKLKNKYWWKNAKMMIILGVICVIV LSELDDRADALQAGASQFETSAAKLKRKYWWKNLKMMI ILGVICAI I LSELDDRADALQAGASQFETSAAKLKRKYWWKNLKMMI ILGVICA I I LSELDDRADALQAGASQFETSAAKLKRKYWWKNLKMMI ILGVICA I I LSELDDRADALQAGASQFETSAAKLKRKYWWKNLKMMIILGVICAI I LSELDDRADALQAGASQFETSAAKLKRKYWWKNLKMMI ILGVICA I I LSELDDRADALQAGASQFETSAAKLKRKYWWKNLKMMI ILGVICA I । LSELDDRTDALQAGASQFETSAAKLKRKYWWKNMKMMI IMGVMCA I I LSELDDRADALQAGASQFETSAAKLKRKYWWKNLKMMI IMGVICA I । LSELDDRADALQAGASQFESSAAKL KNKYWWKNMKMMI IMG IMG I I L LSELDDRADALQAGASVFESSAAKLKRKYWWKNCKMMIMLGA ICAI I LSELDDRADALQAGASQFESSAAKLKRKYWWKNCKMMIMLGA ICA I I LSELDDRADALQAGASQFESSAAKLKRKYWWKNCKMMIMLGA I CA I I LSELDDRADALQAGASQFET SAAKLKRKYWWKN CKMWA I L I AVVLVI LSELGERADQLEQGASQFEQQAGKLKRKQWWANMKMMIILGVIAVVL LSELGERADQLEQGASQFEQQAGKLKRKQWWANMKMMI I LGVIAVVL

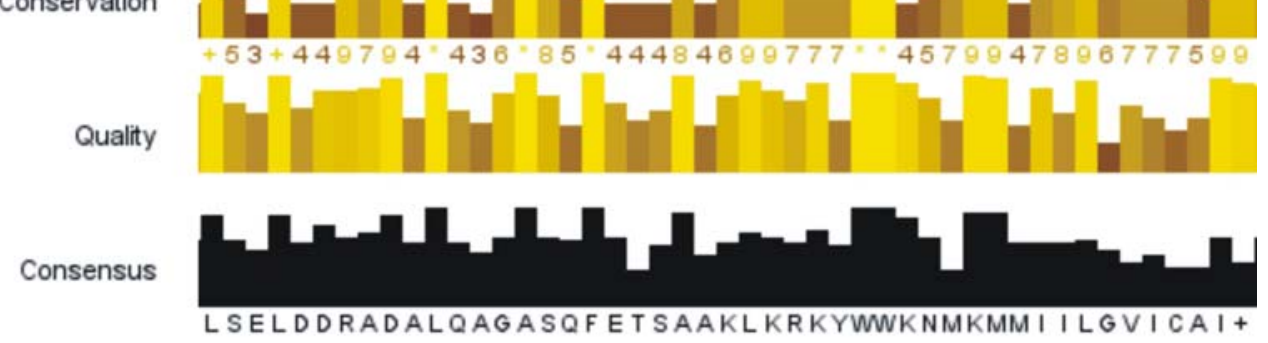

Figure 38. Sequence alignment of C-terminal residues of synaptobrevin homologs. 
Note that the membrane-proximal tryptophan residues are highly conserved across specis, irrespective of the proteins being involved in constitutive or regulated exocytosis. MuMu: Mus musculus, AsPa: Aspergillus parasiticus, AsNig: Aspergillus niger, ScPo: Schizosaccharomyces pombe, SaCe: Saccharomyces cerevisae, KILa: Kluyveromyces lactis, HyJe: Hypocrea jecorina, NeCr: Neurospora crassa, GiZe: Gibberella zeae, HiMe: Hirudo medicinalis, LySt: Lymnea stagnalis, ScJa: Schistosoma japonicum, CaBr: Caenorhabditis briggsae, CaEl: Caenorhabditis elegans, StPu: Strongylocentrotus purpuratus, GaGa: Gallus gallus, DaRe: Danio rerio, RaNo: Rattus norvegicus, HoSa: Homo sapiens, MaMu: Macaca mulatto, OrCu: Orycytolagus cuniculus, XeLa: Xenopus laevis, XeTr: Xenopus tropicalis, DrPs: Drosophila psuedoobscura, DrMe: Drosophila melanogaster. The numbering is derived from the protein with the longest sequence.

Though the function of the polar-aromatic residues is not entirely understood, they might maintain the vertical position of the single transmembrane helix relative to the membrane-water interface (Ridder et al., 2000). Another more likely function for the polar-aromatic residues is that together with the positively charged amino acids, Lys and Arg, they serve to maintain the orientation of the protein (Ridder et al., 2000). Such a pattern has been discerned in many type two single spanning proteins and is not characteristic for synaptbrevin (Arkin and Brunger, 1998; Landolt-Marticorena et al., 1993). The proposed difference between modes of exocytosis assigned to structural difference of the membrane-proximal regions is therefore difficult to reconcile with the fact that this region is highly conserved in nearly all synaptobrevin homologs.

\subsection{Synaptobrevin readily dissociates from synaptophysin to form SNARE complex with soluble syntaxin and SNAP-25}

Synaptobrevin may be regulated by its interaction with other proteins such as synaptophysin. Synaptobrevin is known to be complexed with synaptophysin in the vesicle membrane (Calakos and Scheller, 1994; Edelmann et al., 1995; Washbourne et al., 1995). Synaptophysin together with its homolog, synaptogyrin constitutes about $10 \%$ of total vesicular protein pool (Janz et al., 1999). Interest in synaptophysin and its homolog has dwindled because of the lack of visible phenotype in synaptophysin knock-out (McMahon et al., 1996) and synaptophysin/synaptogyrin double knock-out animals (Janz et al., 1999). It is still possible that these proteins are involved in fine-tuning synaptic transmission or are involved in post-fusion activity.

Although neither the regions of synaptobrevin involved in binding to synaptophysin nor the stoichiomety of the complex is known in detail, binding appears to involve the very C-terminal portion of synaptobrevin, suggesting that at least part of the 
binding is due to interactions between the transmembrane domains (Edelmann et al., 1995; Yelamanchili et al., 2005). The trans-membrane region of synaptobrevin is known to form heterodimer with that of syntaxin via contiguous areas of interfacial residues (Laage et al., 2000; Margittai et al., 1999). The transmembrane region of SNAREs is considered to be important for fusion because SNAREs geranylgeranylated at their Cterminal end are dominant inhibitors of fusion (Grote et al., 2000) and SNAREs with their trans-membrane region replaced with phospholipids are unable to support fusion (McNew et al., 1999; Melia et al., 2002). Therefore the interaction of synaptobrevin with synaptophysin by trans-membrane region may be functionally relevant.

The synaptobrevin-synaptophysin complex dissociates during exocytosis. This dissociation is mediated by cytoplasmic factors that are activated during calcium influx, i.e. it does not depend on membrane fusion (Reisinger et al., 2004). While it is well established that the interactions of synaptobrevin with synaptophysin and the SNAREs syntaxin 1 and SNAP-25 are mutually exclusive (Edelmann et al., 1995; Pennuto et al., 2002; Yelamanchili et al., 2005), it was not known whether dissociation from synaptophysin is a pre-requisite for SNARE complex formation or whether SNARE binding triggers dissociation of the complex. The data in this thesis is largely in agreement with the second alternative, though the first possibility cannot be completely ruled out.

\subsection{Kinetic modelling of SNARE complex assembly}

Studies on the assembly pathway of soluble SNAREs have led to the conclusion that the 1:1 syntaxin/SNAP-25 complex is a true intermediate in the formation of the ternary SNARE complex (Fasshauer and Margittai, 2004). The kinetics of assembly of SNARE complex and its intermediates on membranes, where they are naturally found in the cell, has not been determined.

The reaction on the membrane qualitatively mimics that of soluble SNAREs: increasing the amount of SNAP-25 enhances the rate of complex formation. The overall reaction rate on membranes is, however, significantly lower than that of soluble SNAREs, raising the possibility that the proteins may behave differently on membranes.

Two solutions of reaction rates for the intermediates and product were arrived at by kinetic simulations of SNAP-25 titration experiments, referred to as solution 1 and solution 2. In solution 1, the formation and dissociation of syntaxin/SNAP-25 1:1 
acceptor was similar to that of the published values for soluble SNAREs. In marked contrast to the soluble proteins, the $\operatorname{syntaxin}_{2} / \mathrm{SNAP}-25$ binary complex was considerably suppressed during the reaction. The binding of synaptobrevin to syntaxin/SNAP-25, though twenty times faster than that of syntaxin, was still considerably slow. This means that synaptobrevin in membranes, though not inhibited, is greatly retarded from complex formation with the syntaxin/SNAP-25 acceptor complex. It is conceivable that this retardation in synaptobrevin binding to syntaxin/SNAP-25 is due to synaptobrevin dimerization or as suggested by Kweon et al (Kweon et al., 2003b), the $33^{\circ}$ tilt into the membrane mediated by the membrane-proximal tryptophan residues of synaptobrevin renders it incapable of contacting its binding partners. The model proposed by Shin and colleagues implies that relieving the membrane block imposed by tryptophans by small polar residues would considerably enhance the rate of reaction. However, in this study, the rate of complex formation by wild-type synaptobrevin and W89S W90S Synaptobrevin was comparable. A possibility for the slow rate of complex formation is that synaptobrevin is inaccessible due to dimerization. However, synaptobrevin dimerization is an unlikely possibility for the slow rate of complex formation because two successive studies indicated that dimerization is a relatively weak interaction with a dissociation constant of $10 \mathrm{mM}$, rendering it mechanistically irrelevant (Bowen et al., 2002; Kroch and Fleming, 2006).

In the second solution, the $\mathrm{K}_{\text {on }}$ for syntaxin/SNAP-25 and $\operatorname{syntaxin}_{2} / \mathrm{SNAP}-25$ were comparable to that of soluble proteins. The binding rate of synaptobrevin to the syntaxin/SNAP-25 acceptor complex was rapid (on the order of $10^{5}$ to $10^{6} \mathrm{M}^{-1} \mathrm{~s}^{-1}$ ). The $\mathrm{K}_{\text {off }}$ for syntaxin/SNAP-25 complex being 100 times faster than that of the proteins in solution, the overall reaction rate appeared to be very slow. Two aspects of solution 2 are especially noteworthy. One, the syntaxin/SNAP-25 acceptor complex appears to be shortlived in the presence of synaptobrevin-containing liposomes. The concentration profile of reaction intermediates and products revealed that this complex does not pre-dominate in the reaction. Two, the binding of synaptobrevin to the syntaxin/SNAP-25 acceptor complex was very rapid. When the synaptobrevin-containing liposomes were solubilized by detergent, the rate of reaction was only moderately enhanced suggesting that synaptobrevin's interaction with the membrane (Kweon et al., 2003a) may not affect its reactivity. Moreover, when a syntaxin/SNAP-25 complex stabilised by the C-terminal 
fragment of synaptobrevin (synaptobrevin 42-96) was added to synaptobrevin bearing liposomes, rapid binding of synaptobrevin to this complex was observed (not shown) suggesting that synaptobrevin in liposomes is fully active and capable of engaging soluble partners in a complex.

Together, this study establishes that synaptobrevin is capable of engaging its cognate binding partners in a complex. The kinetic simulations and deconvolution of SNAP-25 titrations experiments carry forward the view that synaptobrevin, even on membranes, binds to the syntaxin/SNAP-25 1:1 acceptor complex. It is still not clear though, whether membrane fusion in vivo proceeds after an inhibitory block is removed from partially zipped complexes (Figure 38, path 1) or whether complex formation nucleating at the N-terminal ends drives SNAREs into a full complex resulting in fusion (Figure 38, path 2). The former proposal is supported by studies involving clostridial neurotoxins: toxins that normally target N-terminal regions of SNAREs were ineffective in blocking nerutransmitter release because the target sites were occluded in the complex but toxins that target the C-terminal regions of SNAREs effectively blocked exocytosis (Hua et al., 1998). A potential weakness of this model is that no molecule that selectively blocks SNAREs in a partially zippered state has been identified. If the syntaxin/SNAP-25 complex in vivo is labile, as assessed by solution 2 kinetic simulation in this study, it is probable that the syntaxin/SNAP-25 complex is stabilised in vivo by an accessory factor or by the membrane itself. The stabilisation on the membrane may be mediated by the trans-membrane region of syntaxin and palmitoylation on the SNAP-25 linker region. However, the kinetics of in vitro reconstituted fusion assays has been on a minutes to hours time-scale, begging askance for a model in which stability is conferred upon solely by the membrane. When syntaxin/SNAP-25 complex was stabilised by c-terminal synaptobrevin (aa 42-96), rapid fusion was observed (Pobbati, $\mathrm{PhD}$ thesis). It is therefore more likely that in vivo, the syntaxin/SNAP-25 complex is stabilised by another protein. In the primed state of the vesicle, synaptobrevin may be prevented by this protein from interacting with syntaxin/SNAP-25. Upon calcium entry, the regulatory factors preventing SNAREs from engaging would dissociate from syntaxin/SNAP-25 and rapid complex formation leading to membrane fusion would occur. 


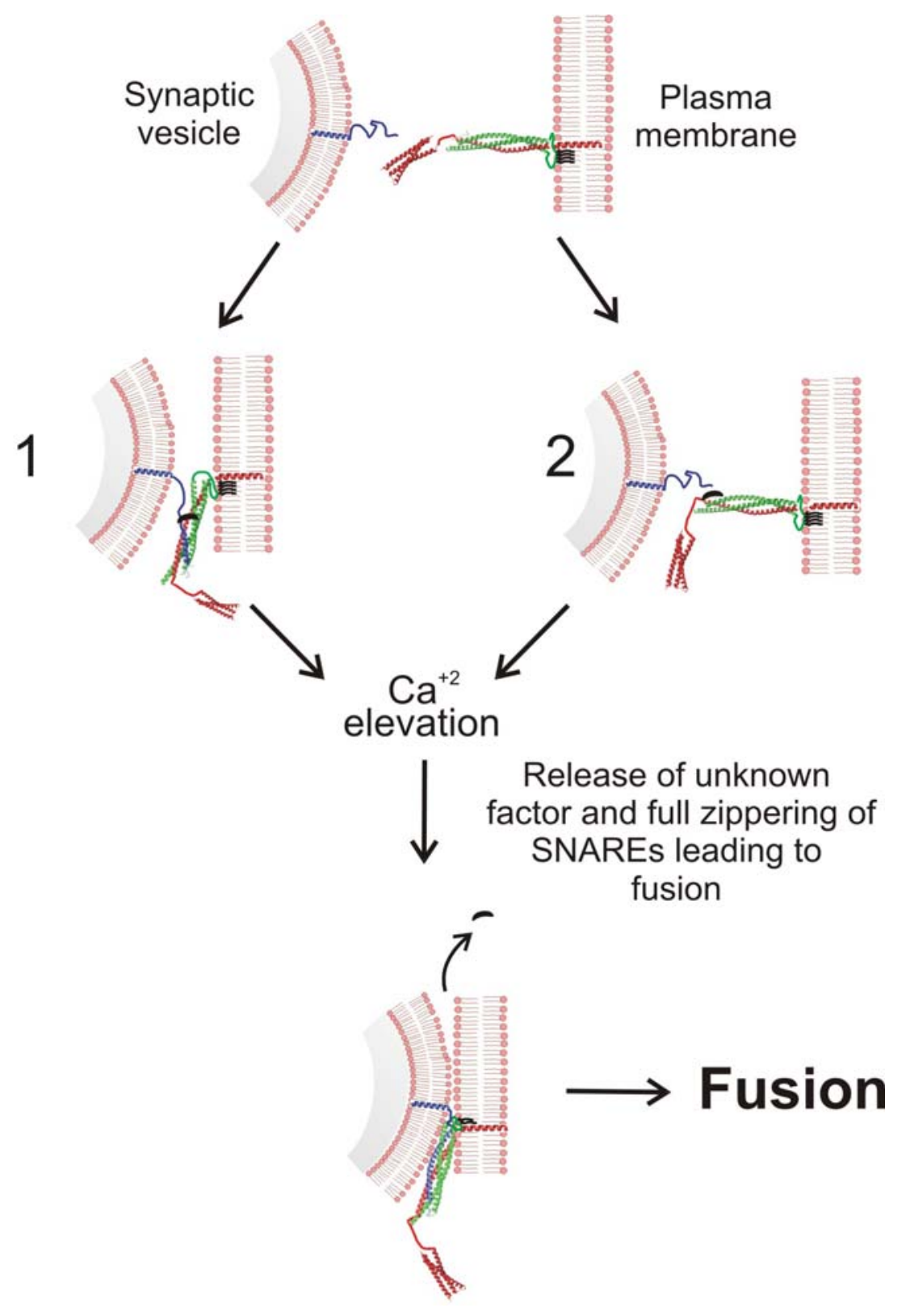

Figure 39. Putative models for synaptobrevin binding to the syntaxin/SNAP-25 acceptor complex.

The kinetic scheme of SNARE complex assembly postulates a syntaxin/SNAP-25 heterodimer being a receptor for synaptobrevin. The binding of synaptobrevin to the syntaxin/SNAP-25 acceptor complex nucleates at the N-terminal ends but is blocked midway by constraints imposed by an unknown factor (1). Calcium elevation would drive out the unknown factor and full zippering of the complex would lead to fusion. Alternatively, the nucleation of the syntaxin/SNAP-25 acceptor complex with synaptobrevin is blocked by an unknown factor, which also stabilizes the acceptor complex (2). Upon calcium elevation, the unknown factor would depart allowing rapid onset of synaptobrevin binding to the acceptor complex, zippering all the way to the C-terminal end of the SNARE motifs, transducing energy though the linker regions and causing membrane fusion. 


\subsection{Anti-ternary complex antibodies: new tools for studying SNARE complexes}

Inside the cell, SNARE complexes are amenable to visualization by FRET when the SNAREs are tagged to a fluorescent protein (An and Almers, 2004). In biochemical experiments, the amount of SNARE complex is estimated by the stoichiometry of individual SNAREs (Fasshauer et al., 1997b). The monoclonal antibody cl 69.1 (Edelmann et al., 1995) directed against synaptobrevin can also recognise synaptobrevin in the complex although by using this antibody within a cell, one cannot distinguish between synaptobrevin and ternary SNARE complex. Thus in immunocytochemical experiments, direct recognition of the ternary SNARE complex without genetic manipulation has not been possible. In order to be able to correctly estimate and visualise SNARE complexes within a cell, three monoclonal antibodies directed against the SNARE complex were successfully raised and characterised.

The inhibition of disassembly of SNAREs by NSF/ $\alpha$-SNAP by the antibodies will probably be useful in functional assays. Three $\alpha$-SNAP molecules have been reported to bind to the SNARE complex (Marz et al., 2003). It is possible that the antibodies block the binding of $\alpha$-SNAP to the SNARE complex since certain level of disassembly was possible when all the three antibodies were added separately, it is suggestive of the disassembly machinery functioning even if two $\alpha$-SNAP molecules are bound to the SNARE complex. Together, these data suggest that disassembly occurs by a robust mechanism and the disassembly machinery is not selective for either end of the complex.

\subsection{SNARE complexes dissociate immediately after exocytosis}

Vesicle fusion with the plasma membrane leads to cis-SNARE complexes on the membrane. These complexes are disassembled into their monomeric components by the NSF/ $\alpha-S N A P$ machinery. Since SNARE complexes are also found on synaptic vesicular membranes (Otto et al., 1997), it is not known at which stage of the exo-endocytosis cycle the disassembly of SNARE complex occurs. Syntaxin-1 and SNAP-25, components of the 
plasma membrane have been found on synaptic vesicles (Koh et al., 1993; WalchSolimena et al., 1995). However, this internal fraction of syntaxin 1 is excluded from synaptic vesicles that undergo activity dependent recycling (Mitchell and Ryan, 2004).

Südhof and colleagues reported that under resting conditions, the amount of SNARE complexes and the readily releasable vesicle pool in superfused synaptosomes increases when it is treated with NEM implying that SNARE complexes are assembled prior to release (Lonart and Sudhof, 2000). In the present study, however, the amount of SNARE complexes in stimulated and unstimulated synaptosomes was comparable suggesting that SNARE complexes disassemble rapidy following exocytosis. It cannot be ruled out, however, that complexes form after solubilization of synaptosomes.

Immunostaining of a synaptic vesicle enriched fraction (LP2) showed the presence of SNARE complexes on a majority of vesicles although the copy number of SNARE complexes per vesicle was much reduced when compared with more abundant vesicleresident proteins. Furthermore, in immunoblots of various sub-cellular fractions, significant amount of SNARE complexes on vesicles were found. These data support previous reports of SNARE complexes on vesicles (Otto et al., 1997), though it cannot be ruled out that SNARE complexes form on isolated vesicles.

Together, these findings suggest that the number of SNARE complexes in a cell is tightly controlled and that due to rapid endocytosis, some of the cis-complexes may be internalized. 


\section{Summary and Conclusions}

The data in this thesis show that the cytoplasmic domain of synaptobrevin is reactive when the protein is membrane-anchored in liposomes as well as in its native resident membrane, laying to rest any speculation about its apparent refractory nature. I have amply demonstrated that the neuronal SNAREs are constitutively active, i.e. there is no need for an energy-consuming activation step prior to fusion, with the probable exception of disassembly mediated by NSF to make monomeric SNAREs available for fusion. This does not exclude the possibility that SNAREs are directly controlled by interaction with other proteins. These interactions cannot be very tight, and such control proteins may rapidly dissociate during the late stages of exocytosis. That synaptobrevin dissociates from synaptophysin to form the SNARE complex, as was clearly demonstrated in this work, is a clear instance of this premise. Weak binding of regulators is favourable since the energy costs for dissociation of the regulators would be low, thus preserving the energy stored in the unfolded free SNARE motifs for the execution of the fusion reaction. Thus, it would be most economical to control the initial contact of the SNARE partners. However, since the energy landscape of the $\mathrm{N} \rightarrow \mathrm{C}$ terminal assembly pathway is not yet known, it is conceivable that SNARE assembly may be arrested after initial trans-contact has been established. Such a scenario is very attractive for neuronal exocytosis because it would allow very fast triggering of membrane merger.

The assembly of SNAREs on membranes follows a pathway similar to that of the soluble versions of the proteins. SNARE complex formation is greatly retarded when syntaxin with the intact $\mathrm{N}$-terminal domain $\left(\mathrm{H}_{\mathrm{abc}}\right.$ domain) is used instead of syntaxin with only the SNARE motif. Moreover, synaptobrevin binds to the pre-assembled syntaxin/SNAP-25 acceptor complex. Kinetic simulations of the SNAP-25 titrations data provide two alternative explanations for the slow rate of assembly on the membranes: one, the binding of synaptobrevin in membranes to the syntaxin/SNAP-25 acceptor complex is indeed slow and two, synaptobrevin binds to the acceptor complex rapidly but the stability of the acceptor complex is compromised. The latter possibility is amply supported by other experimental findings; for instance, synaptobrevin in membranes binds rapidly to an acceptor complex stabilised by a C-terminal peptide.

SNARE complex formation on liposomes reconstituted with synaptobrevin is very robust, largely unaffected by endogenous and extraneous factors. The complex formation 
rate on synaptic vesicles and reconstituted liposomes is comparable. The complex formation rate, however, is enhanced in the presence of weak counter ions, which is typical of protein-protein interactions. I also demonstrated that complex formation on synaptic vesicles is unaffected by calcium, calling into question the proposed role of the calcium sensor, synaptotagmin I (Hu et al., 2002), in relieving the proposed membraneblock of synaptobrevin (Kweon et al., 2003b).

In the second part of the study, three monoclonal antibodies which selectively recognize the ternary SNARE complex but not the monomers were successfully raised and characterised. The binding sites of the antibodies were mapped by immunoblotting for a range of SNARE complexes carrying either a replacement of one of the partners or truncations of one of the SNAREs from either the N-terminal or the C-terminal end. Whereas one of the antibodies depended more on the stability of the N-terminal end of the complex for optimum binding, two antibodies preferred the C-terminal end for optimum binding. The ternary complex has a typical plasma membrane staining and was largely specific for the complex, as assessed by labelling studies on plasma-membrane sheets.

In a functional assay designed to monitor disassembly by NSF/ $\alpha$-SNAP, each of the three antibodies blocked disassembly to a certain extent but appreciable block was possible only when two antibodies, one recognizing the N-terminal end and the other recognizing the $\mathrm{C}$-terminal end, were used in concert. These data indicate that the disassembly process is very robust and the disassembly machinery does not have a preference for either end of the complex. However, in a permeabilized PC-12 cell assay designed to monitor release, the antibodies neither retarded nor potentiated release, indicating that this assay cannot monitor release in subsequent rounds of fusion since the assay runs down relatively fast. Since the antibodies block disassembly of the SNARE complex by NSF/ $\alpha$-SNAP, given a stable system that monitors release for many rounds of exo-endocytosis, a phenotype similar to the comatose mutation in Drosophila (Pallanck et al., 1995) would probably be discernible.

The final experiments dealt with determining the status of SNARE complexes in resting and stimulated pre-synaptic nerve terminals. Interestingly, the total amount of SNARE complex in both the resting and the stimulated synaptosomes was comparable, indicating that SNARE complexes are rapidly disassembled after exocytosis has occurred. It is conceivable that endocytosis may take place with a fraction of the cis-SNARE 
complexes un-disassembled. Indeed, imaging of isolated vesicles indicated that a majority of the vesicles carried a few copies of cis-SNARE complexes. These findings were substantiated by immunoblots of sub-cellular fractions of the rat brain showing significant amount of SNARE complexes in the vesicular fractions. However, it cannot be ruled out that SNARE complexes form on isolated organelles in vitro. 


\section{Abbreviations and symbols}

\begin{tabular}{|c|c|}
\hline$\AA$ & Angstrom \\
\hline aa & Amino acid \\
\hline APS & Ammonium persulfate \\
\hline ATP & Adenosine triphosphate \\
\hline a.u. & Arbitrary units \\
\hline BSA & Bovine serum albumine \\
\hline $\mathrm{cmc}$ & Critical micelle concentration \\
\hline DOPS & 1,2-Dioleoyl-sn-Glycero-3-(Pospho-L-Serine) \\
\hline DTT & Dithiothreitol \\
\hline DNA & Deoxyribonucleic acid \\
\hline E.coli & Escherichia coli \\
\hline EDTA & Ethylendiaminetetraacetic acid \\
\hline EPR & Electron paramagnetic resonance \\
\hline FFF & Free flow fractionation \\
\hline FRET & Förster resonance energy transfer \\
\hline GST & Glutathion-S-transferase \\
\hline GTP & Guanosine triphosphate \\
\hline GTPase & GTP hydrolysing enzyme \\
\hline IgG & Immunoglobulin $\mathrm{G}$ \\
\hline IPTG & Isopropyl- $\beta$-D-thiogalactoside \\
\hline $\mathrm{kDa}$ & Kilo Dalton \\
\hline LB & Luria Bertani medium \\
\hline $\mathrm{mS}$ & Milli Siemens \\
\hline MWCO & Molecular weight cut off \\
\hline NEM & N-ethyl-maleimide \\
\hline Ni-NTA & Nickel-nitrilo-triacetate \\
\hline NSF & N-ethylmaleimide-sensitive factor \\
\hline PAGE & Polyacrylamide gel electrophoresis \\
\hline PBS & Phosphate buffered saline \\
\hline PCR & Polymerase chain reaction \\
\hline
\end{tabular}




$\begin{array}{ll}\text { PMSF } & \text { Phenymethylsulfonylflouride } \\ \text { POPC } & \text { 1-Palmitoy-2-Oleoyl-sn-Glycero-3-Phosphocholine } \\ \text { pH } & \text { Negative logarithm of } \mathrm{H}^{+} \text {concentration } \\ \text { rpm } & \text { Revolutions per minute } \\ \text { RT } & \text { Room temperature } \\ \text { S } & \text { Svedberg } \\ \text { SDS } & \text { Sodium dodecyl sulfate } \\ \text { SEM } & \text { Standard error of the mean } \\ \text { SD } & \text { Standard deviation } \\ \text { SNAP } & \text { Soluble-NSF-attachment protein } \\ \text { SNAP-25 } & \text { Synaptosome associated protein of 25 kDa } \\ \text { SNARE } & \text { SNAP-receptor } \\ \text { TB } & \text { Terrific broth } \\ \text { TEMED } & \text { N,N,N',N'-Tetramethylethylene diamine } \\ \text { Tris } & \text { Trisand -aminomethane } \\ \text { V/v } & \text { Volume/volume } \\ \text { W/v } & \text { Weigth/volume }\end{array}$




\section{List of Publications}

Siddiqui, T J, Jahn R, Fasshauer D, ' Membrane-anchored synaptobrevin is active and readily engages in SNARE complexes' (submitted)

Siddiqui, T J, Rizzoli S O, Thorsten Lang, Fasshauer D, Jahn R. 'Anti-ternary SNARE complex antibodies: new tools for dissecting neuro-exocytosis' (manuscript in preparation) 


\section{Curriculum vitae}

\section{Personal information}

Name:

Tabrez Jamal Siddiqui

Date of birth: $\quad 10^{\text {th }}$ of April, 1976

Place of birth: Calcutta, India

Nationality: Indian

\section{Education}

2003 - Present:

$\mathrm{Ph}$.D. student of the International Graduate Program in Molecular Biology, Georg-August-Universität, Göttingen, Germany at the Department of Neurobiology, Max Planck Institute for Biophysical Chemstry, Göttingen, Germany

2001 - 2003:

International Master's degree in Molecular Biology, International Graduate Program in Molecular Biology, Georg-August-Universität, Göttingen, Germany.

1997-2000:

BSc. (Hons.) Biochemistry, Aligarh Muslim University, Aligarh, India 


\section{Bibliography}

Ahnert-Hilger, G., Stecher, B., Beyer, C., and Gratzl, M. (1993). Exocytotic membrane fusion as studied in toxin-permeabilized cells. Methods Enzymol 221, 139-149.

Akert, K., Moor H, Pfenninger K (1971). Synaptic fine structure. Adv Cytopharmacol 1, $273-290$.

An, S. J., and Almers, W. (2004). Tracking SNARE Complex Formation in Live Endocrine Cells. Science 306, 1042-1046.

Antonin, W., Dulubova, I., Arac, D., Pabst, S., Plitzner, J., Rizo, J., and Jahn, R. (2002a). The N-terminal domains of syntaxin 7 and vtilb form three-helix bundles that differ in their ability to regulate SNARE complex assembly. J Biol Chem 277, 36449-36456.

Antonin, W., Fasshauer, D., Becker, S., Jahn, R., and Schneider, T. R. (2002b). Crystal structure of the endosomal SNARE complex reveals common structural principles of all SNAREs. Nat Struct Biol 9, 107-111.

Arkin, I. T., and Brunger, A. T. (1998). Statistical analysis of predicted transmembrane alpha-helices. Biochim Biophys Acta 1429, 113-128.

Augustin, I., Rosenmund, C., Sudhof, T. C., and Brose, N. (1999). Munc13-1 is essential for fusion competence of glutamatergic synaptic vesicles. Nature 400, 457-461.

Baba, T., Sakisaka, T., Mochida, S., and Takai, Y. (2005). PKA-catalyzed phosphorylation of tomosyn and its implication in $\mathrm{Ca} 2+$-dependent exocytosis of neurotransmitter. J Cell Biol 170, 1113-1125.

Bacia, K., Schuette, C. G., Kahya, N., Jahn, R., and Schwille, P. (2004). SNAREs Prefer Liquid-disordered over "Raft" (Liquid-ordered) Domains When Reconstituted into Giant Unilamellar Vesicles. J Biol Chem 279, 37951-37955.

Bai, J., Wang, C. T., Richards, D. A., Jackson, M. B., and Chapman, E. R. (2004). Fusion pore dynamics are regulated by synaptotagmin*t-SNARE interactions. Neuron 41, 929942.

Bark, I. C., Hahn, K. M., Ryabinin, A. E., and Wilson, M. C. (1995). Differential expression of SNAP-25 protein isoforms during divergent vesicle fusion events of neural development. Proc Natl Acad Sci U S A 92, 1510-1514.

Baumert, M., P R Maycox, F Navone, P De Camilli, and R Jahn (1989 ). Synaptobrevin: an integral membrane protein of 18,000 daltons present in small synaptic vesicles of rat brain. EMBO J February 379-384. 
Bibliography

Bennett, M., Calakos N, Scheller RH. (1992 ). Syntaxin: a synaptic protein implicated in docking of synaptic vesicles at presynaptic active zones. Science 257(5067), 255-259. .

Betz, A., Okamoto, M., Benseler, F., and Brose, N. (1997). Direct interaction of the rat unc-13 homologue Munc13-1 with the N terminus of syntaxin. J Biol Chem 272, 25202526.

Bhattacharya, S., Stewart, B. A., Niemeyer, B. A., Burgess, R. W., McCabe, B. D., Lin, P., Boulianne, G., O'Kane, C. J., and Schwarz, T. L. (2002). Members of the synaptobrevin/vesicle-associated membrane protein (VAMP) family in Drosophila are functionally interchangeable in vivo for neurotransmitter release and cell viability. Proc Natl Acad Sci U S A 99, 13867-13872.

Blasi, J., Chapman, E. R., Link, E., Binz, T., Yamasaki, S., Camilli, P. D., Sudhof, T. C., Niemann, H., and Jahn, R. (1993). Botulinum neurotoxin A selectively cleaves the synaptic protein SNAP-25. Nature 365, 160-163.

Blasi, J., E R Chapman, S Yamasaki, T Binz, H Niemann, and R Jahn (1993). Botulinum neurotoxin $\mathrm{C} 1$ blocks neurotransmitter release by means of cleaving HPC-1/syntaxin. EMBO J Dec;12, 4821-4828.

Bock, J. B., Matern, H. T., Peden, A. A., and Scheller, R. H. (2001). A genomic perspective on membrane compartment organization. Nature 409, 839-841.

Borisovska, M., Zhao, Y., Tsytsyura, Y., Glyvuk, N., Takamori, S., Matti, U., Rettig, J., Sudhof, T., and Bruns, D. (2005). v-SNAREs control exocytosis of vesicles from priming to fusion. EMBO J, sj.emboj. 7600696.

Bowen, M. E., Engelman, D. M., and Brunger, A. T. (2002). Mutational analysis of synaptobrevin transmembrane domain oligomerization. Biochemistry 41, 15861-15866.

Bradford, M. M. (1976). A rapid and sensitive method for the quantitation of microgram quantities of protein utilizing the principle of protein-dye binding. Anal Biochem 72, 248254.

Brandhorst, D., Zwilling, D., Rizzoli, S. O., Lippert, U., Lang, T., and Jahn, R. (2006). Homotypic fusion of early endosomes: SNAREs do not determine fusion specificity. Proc Natl Acad Sci U S A.

Breidenbach, M. A., and Brunger, A. T. (2005). New insights into clostridial neurotoxinSNARE interactions. Trends in Molecular Medicine 11, 377-381.

Broadie, K., Prokop A, Bellen HJ, O'Kane CJ, Schulze KL, Sweeney ST (1995). Syntaxin and synaptobrevin function downstream of vesicle docking in Drosophila. Neuron Sep15 663-673. 
Bibliography

Brooks, J. C., and Treml, S. (1984). Effect of trifluoperazine and calmodulin on catecholamine secretion by saponin-skinned cultured chromaffin cells. Life Sci 34, 669674.

Brose, N., Petrenko, A. G., Sudhof, T. C., and Jahn, R. (1992). Synaptotagmin: a calcium sensor on the synaptic vesicle surface. Science 256, 1021-1025.

Brunger, A. T., and Ernst, J. A. (2002). High resolution structure, stability, and synaptotagmin binding of a truncated neuronal SNARE complex. J Biol Chem 20, 20.

Burgoyne, R. D. (1984). Mechanisms of secretion from adrenal chromaffin cells. Biochim Biophys Acta 779, 201-216.

Calakos, N., Bennett, M. K., Peterson, K. E., and Scheller, R. H. (1994). Protein-protein interactions contributing to the specificity of intracellular vesicular trafficking. Science 263, 1146-1149.

Calakos, N., and Scheller, R. H. (1994). Vesicle-associated membrane protein and synaptophysin are associated on the synaptic vesicle. J Biol Chem 269, 24534-24537.

Canvin, A. T., and Buhr, M. M. (1989). Effect of temperature on the fluidity of boar sperm membranes. J Reprod Fertil 85, 533-540.

Cao, X., and Barlowe, C. (2000). Asymmetric requirements for a Rab GTPase and SNARE proteins in fusion of COPII vesicles with acceptor membranes. J Cell Biol 149, 55-66.

Chamberlain, L. H., Roth, D., Morgan, A., and Burgoyne, R. D. (1995). Distinct effects of alpha-SNAP, 14-3-3 proteins, and calmodulin on priming and triggering of regulated exocytosis. J Cell Biol 130, 1063-1070.

Chapman, E. R. (2002). Synaptotagmin: a $\mathrm{Ca}(2+)$ sensor that triggers exocytosis? Nat Rev Mol Cell Biol 3, 498-508.

Chapman, E. R., Hanson, P. I., An, S., and Jahn, R. (1995). Ca2+ regulates the interaction between synaptotagmin and syntaxin 1. J Biol Chem 270, 23667-23671.

Chen, X., Tang, J., Sudhof, T. C., and Rizo, J. (2005). Are neuronal SNARE proteins Ca2+ sensors? J Mol Biol 347, 145-158.

Chen, X., Tomchick, D. R., Kovrigin, E., Arac, D., Machius, M., Sudhof, T. C., and Rizo, J. (2002). Three-dimensional structure of the complexin/SNARE complex. Neuron 33, 397-409.

Chen, Y., Xu, Y., Zhang, F., and Shin, Y. K. (2004). Constitutive versus regulated SNARE assembly: a structural basis. Embo J. 
Bibliography

Chen, Y. A., Scales, S. J., and Scheller, R. H. (2001). Sequential SNARE assembly underlies priming and triggering of exocytosis. Neuron 30, 161-170.

Chen, Y. A., and Scheller, R. H. (2001). SNARE-mediated membrane fusion. Nat Rev Mol Cell Biol 2, 98-106.

Constable, J. R., Graham, M. E., Morgan, A., and Burgoyne, R. D. (2005). Amisyn regulates exocytosis and fusion pore stability by both syntaxin-dependent and syntaxinindependent mechanisms. J Biol Chem 280, 31615-31623.

Davis, A. F., Bai, J., Fasshauer, D., Wolowick, M. J., Lewis, J. L., and Chapman, E. R. (1999). Kinetics of synaptotagmin responses to Ca2+ and assembly with the core SNARE complex onto membranes. Neuron 24, 363-376.

de Haro, L., Ferracci, G., Opi, S., Iborra, C., Quetglas, S., Miquelis, R., Leveque, C., and Seagar, M. (2004). Ca2+/calmodulin transfers the membrane-proximal lipid-binding domain of the v-SNARE synaptobrevin from cis to trans bilayers. Proc Natl Acad Sci U S A $101,1578-1583$.

De Haro, L., Quetglas, S., Iborra, C., Leveque, C., and Seagar, M. (2003). Calmodulindependent regulation of a lipid binding domain in the v-SNARE synaptobrevin and its role in vesicular fusion. Biol Cell 95, 459-464.

Dower, W. J., Miller, J. F., and Ragsdale, C. W. (1988). High efficiency transformation of E. coli by high voltage electroporation. Nucleic Acids Res 16, 6127-6145.

Dulubova, I., Sugita, S., Hill, S., Hosaka, M., Fernandez, I., Sudhof, T. C., and Rizo, J. (1999). A conformational switch in syntaxin during exocytosis: role of munc18. Embo J $18,4372-4382$.

Edelmann, L., Hanson, P. I., Chapman, E. R., and Jahn, R. (1995). Synaptobrevin binding to synaptophysin: a potential mechanism for controlling the exocytotic fusion machine. Embo J 14, 224-231.

Eisel, U., Jarausch, W., Goretzki, K., Henschen, A., Engels, J., Weller, U., Hudel, M., Habermann, E., and Niemann, H. (1986). Tetanus toxin: primary structure, expression in E. coli, and homology with botulinum toxins. Embo J 5, 2495-2502.

Fairweather, N. F., Lyness, V. A., Pickard, D. J., Allen, G., and Thomson, R. O. (1986). Cloning, nucleotide sequencing, and expression of tetanus toxin fragment $\mathrm{C}$ in Escherichia coli. J Bacteriol 165, 21-27.

Fasshauer, D. (2003). Structural insights into the SNARE mechanism. Biochim Biophys Acta 1641, 87-97. 
Fasshauer, D., Antonin, W., Margittai, M., Pabst, S., and Jahn, R. (1999). Mixed and noncognate SNARE complexes. Characterization of assembly and biophysical properties. J Biol Chem 274, 15440-15446.

Fasshauer, D., Antonin, W., Subramaniam, V., and Jahn, R. (2002). SNARE assembly and disassembly exhibit a pronounced hysteresis. Nat Struct Biol 9, 144-151.

Fasshauer, D., Bruns, D., Shen, B., Jahn, R., and Brunger, A. T. (1997a). A structural change occurs upon binding of syntaxin to SNAP-25. J Biol Chem 272, 4582-4590.

Fasshauer, D., Eliason, W. K., Brunger, A. T., and Jahn, R. (1998a). Identification of a minimal core of the synaptic SNARE complex sufficient for reversible assembly and disassembly. Biochemistry 37, 10354-10362.

Fasshauer, D., and Margittai, M. (2004). A Transient N-terminal Interaction of SNAP-25 and Syntaxin Nucleates SNARE Assembly. J Biol Chem 279, 7613-7621.

Fasshauer, D., Otto, H., Eliason, W. K., Jahn, R., and Brunger, A. T. (1997b). Structural changes are associated with soluble N-ethylmaleimide- sensitive fusion protein attachment protein receptor complex formation. J Biol Chem 272, 28036-28041.

Fasshauer, D., Sutton, R. B., Brunger, A. T., and Jahn, R. (1998b). Conserved structural features of the synaptic fusion complex: SNARE proteins reclassified as Q- and RSNAREs. Proc Natl Acad Sci U S A 95, 15781-15786.

Fergestad, T., Wu, M. N., Schulze, K. L., Lloyd, T. E., Bellen, H. J., and Broadie, K. (2001). Targeted mutations in the syntaxin H3 domain specifically disrupt SNARE complex function in synaptic transmission. J Neurosci 21, 9142-9150.

Fernandez, I., Ubach, J., Dulubova, I., Zhang, X., Sudhof, T. C., and Rizo, J. (1998). Three-Dimensional Structure of an Evolutionarily Conserved N-Terminal Domain of Syntaxin 1A. Cell 94, 841-849.

Fiebig, K. M., Rice, L. M., Pollock, E., and Brunger, A. T. (1999). Folding intermediates of SNARE complex assembly. Nat Struct Biol 6, 117-123.

Fischer von Mollard, G., Sudhof, T. C., and Jahn, R. (1991). A small GTP-binding protein dissociates from synaptic vesicles during exocytosis. Nature 349, 79-81.

Fujita, Y., Shirataki, H., Sakisaka, T., Asakura, T., Ohya, T., Kotani, H., Yokoyama, S., Nishioka, H., Matsuura, Y., Mizoguchi, A., et al. (1998). Tomosyn: a syntaxin-1-binding protein that forms a novel complex in the neurotransmitter release process. Neuron 20, 905-915. 
Geppert, M., Goda, Y., Hammer, R. E., Li, C., Rosahl, T. W., Stevens, C. F., and Sudhof, T. C. (1994). Synaptotagmin I: a major Ca2+ sensor for transmitter release at a central synapse. Cell 79, 717-727.

Gonzalez, L. C., Jr., Weis, W. I., and Scheller, R. H. (2001). A Novel SNARE N-terminal Domain Revealed by the Crystal Structure of Sec22b. J Biol Chem 276, 24203-24211.

Graf, C. T., Riedel, D., Schmitt, H. D., and Jahn, R. (2005). Identification of functionally interacting SNAREs by using complementary substitutions in the conserved '0' layer. Mol Biol Cell 16, 2263-2274.

Griep, M. A., and McHenry, C. S. (1989). Glutamate overcomes the salt inhibition of DNA polymerase III holoenzyme. J Biol Chem 264, 11294-11301.

Griffin, E. E., Detmer, S. A., and Chan, D. C. (2006). Molecular mechanism of mitochondrial membrane fusion. Biochimica et Biophysica Acta (BBA) - Molecular Cell Research.

Grote, E., Baba, M., Ohsumi, Y., and Novick, P. J. (2000). Geranylgeranylated SNAREs are dominant inhibitors of membrane fusion. J Cell Biol 151, 453-466.

Hanson, M. A., and Stevens, R. C. (2000). Cocrystal structure of synaptobrevin-II bound to botulinum neurotoxin type B at 2.0 A resolution. Nat Struct Biol 7, 687-692.

Hanson, P. I., Heuser, J. E., and Jahn, R. (1997). Neurotransmitter release - four years of SNARE complexes. Curr Opin Neurobiol 7, 310-315.

Hatsuzawa, K., Lang, T., Fasshauer, D., Bruns, D., and Jahn, R. (2003). The R-SNARE motif of tomosyn forms SNARE core complexes with syntaxin 1 and SNAP-25 and down-regulates exocytosis. J Biol Chem 278, 31159-31166.

Hauser, D., Eklund, M. W., Kurazono, H., Binz, T., Niemann, H., Gill, D. M., Boquet, P., and Popoff, M. R. (1990). Nucleotide sequence of Clostridium botulinum C1 neurotoxin. Nucleic Acids Res 18, 4924.

Hay, J. C., and Martin, T. F. (1992). Resolution of regulated secretion into sequential MgATP-dependent and calcium-dependent stages mediated by distinct cytosolic proteins. J Cell Biol 119, 139-151.

Hayashi, T., McMahon, H., Yamasaki, S., Binz, T., Hata, Y., Sudhof, T. C., and Niemann, H. (1994). Synaptic vesicle membrane fusion complex: action of clostridial neurotoxins on assembly. Embo J 13, 5051-5061.

Hazzard, J., Sudhof, T. C., and Rizo, J. (1999). NMR analysis of the structure of synaptobrevin and of its interaction with syntaxin. J Biomol NMR 14, 203-207. 
Heiner Niemann, J. B. a. R. J. (1994). Clostridial Neurotoxins: new tools for dissecting exocytosis. Trends in Cell Biology 4.

Hell, J. W., Jahn, R. (1994). Preparation of Synaptic Vesicles from Mammalian Brain. Academic Press, Inc.

Hess, D. T., Slater, T. M., Wilson, M. C., and Skene, J. H. (1992). The 25 kDa synaptosomal-associated protein SNAP-25 is the major methionine-rich polypeptide in rapid axonal transport and a major substrate for palmitoylation in adult CNS. J Neurosci 12, 4634-4641.

Heumann, R., Kachel, V., and Thoenen, H. (1983). Relationship between NGF-mediated volume increase and "priming effect" in fast and slow reacting clones of PC12 pheochromocytoma cells. Role of cAMP. Exp Cell Res 145, 179-190.

Horsnell, W. G., Steel, G. J., and Morgan, A. (2002). Analysis of NSF mutants reveals residues involved in SNAP binding and ATPase stimulation. Biochemistry 41, 52305235.

Hu, K., Carroll, J., Fedorovich, S., Rickman, C., Sukhodub, A., and Davletov, B. (2002). Vesicular restriction of synaptobrevin suggests a role for calcium in membrane fusion. Nature 415, 646-650.

Hu, K., Rickman, C., Carroll, J., and Davletov, B. (2003). A common mechanism for regulation of vesicular SNAREs on phospholipid membranes. Biochem J Pt.

Hu, K., Rickman, C., Carroll, J., and Davletov, B. (2004). A common mechanism for the regulation of vesicular SNAREs on phospholipid membranes. Biochem J 377, 781-785.

Hua, S. Y., Raciborska, D. A., Trimble, W. S., and Charlton, M. P. (1998). Different VAMP/synaptobrevin complexes for spontaneous and evoked transmitter release at the crayfish neuromuscular junction. J Neurophysiol 80, 3233-3246.

Huttner, W. B., Schiebler, W., Greengard, P., and De Camilli, P. (1983). Synapsin I (protein I), a nerve terminal-specific phosphoprotein. III. Its association with synaptic vesicles studied in a highly purified synaptic vesicle preparation. J Cell Biol 96, 13741388.

Inoue, N., Ikawa, M., Isotani, A., and Okabe, M. (2005). The immunoglobulin superfamily protein Izumo is required for sperm to fuse with eggs. Nature 434, 234-238.

Jahn, R., and Grubmuller, H. (2002). Membrane fusion. Curr Opin Cell Biol 14, 488-495. Jahn, R., Lang, T., and Sudhof, T. C. (2003). Membrane fusion. Cell 112, 519-533.

Jahn, R., Schiebler, W., Ouimet, C., and Greengard, P. (1985). A 38,000-Dalton Membrane Protein (p38) Present in Synaptic Vesicles. PNAS 82, 4137-4141. 
Jahn, R., and Sudhof, T. C. (1999). Membrane fusion and exocytosis. Annu Rev Biochem 68, 863-911.

Janz, R., Sudhof, T. C., Hammer, R. E., Unni, V., Siegelbaum, S. A., and Bolshakov, V. Y. (1999). Essential roles in synaptic plasticity for synaptogyrin I and synaptophysin I. Neuron 24, 687-700.

Junge, H. J., Rhee, J. S., Jahn, O., Varoqueaux, F., Spiess, J., Waxham, M. N., Rosenmund, C., and Brose, N. (2004). Calmodulin and Munc13 form a Ca2+ sensor/effector complex that controls short-term synaptic plasticity. Cell 118, 389-401.

Kandel, E. R., Schwartz, J. H. \& Jessell, T. M (2000). Principles of Neural Science (fourth edition). McGraw-Hill Companies.

Karin L. Nicholson, M. M., Rebecca B. Miller, Thomas J Filip, Robert Fairman, and Frederick M. Hughson (1998). Regulation of SNARE complex assembly by an Nterminal domain of the t-SNARE Sso1p. Nature Structural Biology 5, 793-801.

Katz, B. (1969). The Release of Neural Transmitter Substances. Liverpool: Liverpool Univ Press

Katz, B., and Thesleff, S. (1957). On the factors which determine the amplitude of the miniature end-plate potential. J Physiol 137, 267-278.

Katz, L., and Brennwald, P. (2000). Testing the 3Q:1R "rule": mutational analysis of the ionic "zero" layer in the yeast exocytic SNARE complex reveals no requirement for arginine. Mol Biol Cell 11, 3849-3858.

Killian, J. A., and von Heijne, G. (2000). How proteins adapt to a membrane-water interface. Trends in Biochemical Sciences 25, 429-434.

Klenchin, V. A., Kowalchyk, J. A., and Martin, T. F. J. (1998). Large Dense-Core Vesicle Exocytosis in PC12 Cells. Methods 16, 204-208.

Knecht, V., and Grubmuller, H. (2003). Mechanical Coupling via the Membrane Fusion SNARE Protein Syntaxin 1A: A Molecular Dynamics Study. Biophys J 84, 1527-1547.

Koh, S., Yamamoto, A., Inoue, A., Inoue, Y., Akagawa, K., Kawamura, Y., Kawamoto, K., and Tashiro, Y. (1993). Immunoelectron microscopic localization of the HPC-1 antigen in rat cerebellum. J Neurocytol 22, 995-1005.

Koticha, D. K., McCarthy, E. E., and Baldini, G. (2002). Plasma membrane targeting of SNAP-25 increases its local concentration and is necessary for SNARE complex formation and regulated exocytosis. J Cell Sci 115, 3341-3351. 
Bibliography

Kroch, A. E., and Fleming, K. G. (2006). Alternate Interfaces May Mediate Homomeric and Heteromeric Assembly in the Transmembrane Domains of SNARE Proteins. Journal of Molecular Biology 357, 184-194.

Kuzmin, P. I., Zimmerberg, J., Chizmadzhev, Y. A., and Cohen, F. S. (2001). A quantitative model for membrane fusion based on low-energy intermediates. Proceedings Of The National Academy Of Sciences Of The United States Of America 98, 7235-7240.

Kweon, D. H., Kim, C. S., and Shin, Y. K. (2003a). Insertion of the membrane proximal region of the neuronal SNARE coiled coil into the membrane. J Biol Chem 15, 15.

Kweon, D. H., Kim, C. S., and Shin, Y. K. (2003b). Regulation of neuronal SNARE assembly by the membrane. Nat Struct Biol 10, 440-447.

Laage, R., and Langosch, D. (1997). Dimerization of the synaptic vesicle protein synaptobrevin (vesicle- associated membrane protein) II depends on specific residues within the transmembrane segment. Eur J Biochem 249, 540-546.

Laage, R., Rohde, J., Brosig, B., and Langosch, D. (2000). A conserved membranespanning amino acid motif drives homomeric and supports heteromeric assembly of presynaptic SNARE proteins. J Biol Chem 275, 17481-17487.

Laemmli, U. K. (1970). Cleavage of structural proteins during the assembly of the head of bacteriophage T4. Nature 227, 680-685.

Lakowicz, J. R. (2000). Principles of Fluorescence Specroscopy (second edition). Kluwer Academic, Plenum Publishers, New York.

Landolt-Marticorena, C., Williams, K. A., Deber, C. M., and Reithmeier, R. A. (1993). Non-random distribution of amino acids in the transmembrane segments of human type I single span membrane proteins. J Mol Biol 229, 602-608.

Lane, S. R., and Liu, Y. (1997). Characterization of the palmitoylation domain of SNAP25. J Neurochem 69, 1864-1869.

Lang, T., Margittai, M., Holzler, H., and Jahn, R. (2002). SNAREs in native plasma membranes are active and readily form core complexes with endogenous and exogenous SNAREs. J Cell Biol 158, 751-760.

Lerman, J. C., Robblee, J., Fairman, R., and Hughson, F. M. (2000). Structural analysis of the neuronal SNARE protein syntaxin-1A. Biochemistry 39, 8470-8479.

Lin, R. C., and Scheller, R. H. (1997). Structural organization of the synaptic exocytosis core complex. Neuron 19, 1087-1094.

Link, E., Edelmann, L., Chou, J. H., Binz, T., Yamasaki, S., Eisel, U., Baumert, M., Sudhof, T. C., Niemann, H., and Jahn, R. (1992). Tetanus toxin action: Inhibition of 
Bibliography

neurotransmitter release linked to synaptobrevin proteolysis. Biochemical and Biophysical Research Communications 189, 1017-1023.

Littleton, J. T., Chapman, E. R., Kreber, R., Garment, M. B., Carlson, S. D., and Ganetzky, B. (1998). Temperature-sensitive paralytic mutations demonstrate that synaptic exocytosis requires SNARE complex assembly and disassembly. Neuron 21, 401-413.

Liu, Y., and Barlowe, C. (2002). Analysis of Sec22p in endoplasmic reticulum/Golgi transport reveals cellular redundancy in SNARE protein function. Mol Biol Cell 13, 33143324.

Loewi, O. (1921). Über humorale Übertragbarkeit der Herznervenwirkung. Pfluegers Arch 189, 201-203.

Lonart, G., and Sudhof, T. C. (2000). Assembly of SNARE core complexes prior to neurotransmitter release sets the readily releasable pool of synaptic vesicles. J Biol Chem 275, 27703-27707.

Manders, E. M., Stap, J., Brakenhoff, G. J., van Driel, R., and Aten, J. A. (1992). Dynamics of three-dimensional replication patterns during the S-phase, analysed by double labelling of DNA and confocal microscopy. J Cell Sci 103 ( Pt 3), 857-862.

Margittai, M., Fasshauer, D., Jahn, R., and Langen, R. (2003a). The Habc domain and the SNARE core complex are connected by a highly flexible linker. Biochemistry 42, 40094014.

Margittai, M., Fasshauer, D., Pabst, S., Jahn, R., and Langen, R. (2001). Homo- and heterooligomeric SNARE complexes studied by site-directed spin labeling. J Biol Chem 276, 13169-13177.

Margittai, M., Otto, H., and Jahn, R. (1999). A stable interaction between syntaxin 1a and synaptobrevin 2 mediated by their transmembrane domains. FEBS Lett 446, 40-44.

Margittai, M., Widengren, J., Schweinberger, E., Schroder, G. F., Felekyan, S., Haustein, E., Konig, M., Fasshauer, D., Grubmuller, H., Jahn, R., and Seidel, C. A. (2003b). Singlemolecule fluorescence resonance energy transfer reveals a dynamic equilibrium between closed and open conformations of syntaxin 1. Proc Natl Acad Sci U S A 100, 1551615521.

Margittai, M., Widengren, J., Schweinberger, E., Schroder, G. F., Felekyan, S., Haustein, E., Konig, M., Fasshauer, D., Grubmuller, H., Jahn, R., and Seidel, C. A. M. (2003c). Single-molecule fluorescence resonance energy transfer reveals a dynamic equilibrium between closed and open conformations of syntaxin 1. PNAS 100, 15516-15521.

Margittai $\mathrm{PhD}$ Thesis, $\mathrm{M}$. 
Marz, K. E., Lauer, J. M., and Hanson, P. I. (2003). Defining the SNARE complex binding surface of alpha-SNAP: implications for SNARE complex disassembly. J Biol Chem 278, 27000-27008.

McMahon, H. T., Bolshakov, V. Y., Janz, R., Hammer, R. E., Siegelbaum, S. A., and Sudhof, T. C. (1996). Synaptophysin, a major synaptic vesicle protein, is not essential for neurotransmitter release. Proc Natl Acad Sci U S A 93, 4760-4764.

McNew, J. A., Parlati, F., Fukuda, R., Johnston, R. J., Paz, K., Paumet, F., Sollner, T. H., and Rothman, J. E. (2000). Compartmental specificity of cellular membrane fusion encoded in SNARE proteins. Nature 407, 153-159.

McNew, J. A., Weber, T., Engelman, D. M., Sollner, T. H., and Rothman, J. E. (1999). The length of the flexible SNAREpin juxtamembrane region is a critical determinant of SNARE-dependent fusion. Mol Cell 4, 415-421.

Melia, T. J., Weber, T., McNew, J. A., Fisher, L. E., Johnston, R. J., Parlati, F., Mahal, L. K., Sollner, T. H., and Rothman, J. E. (2002). Regulation of membrane fusion by the membrane-proximal coil of the t- SNARE during zippering of SNAREpins. J Cell Biol 158, 929-940.

Misura, K. M., Gonzalez, L. C., Jr., May, A. P., Scheller, R. H., and Weis, W. I. (2001a). Crystal structure and biophysical properties of a complex between the $\mathrm{N}$ - terminal SNARE region of SNAP25 and syntaxin 1a. J Biol Chem 276, 41301-41309.

Misura, K. M., Scheller, R. H., and Weis, W. I. (2000). Three-dimensional structure of the neuronal-Sec1-syntaxin 1a complex. Nature 404, 355-362.

Misura, K. M., Scheller, R. H., and Weis, W. I. (2001b). Self-association of the H3 region of syntaxin 1A. Implications for intermediates in SNARE complex assembly. J Biol Chem 276, 13273-13282.

Mitchell, S. J., and Ryan, T. A. (2004). Syntaxin-1A is excluded from recycling synaptic vesicles at nerve terminals. J Neurosci 24, 4884-4888.

Montecucco, C., Schiavo, G., and Pantano, S. (2005). SNARE complexes and neuroexocytosis: how many, how close? Trends Biochem Sci 30, 367-372.

Nagy, A., Baker, R. R., Morris, S. J., and Whittaker, V. P. (1976). The preparation and characterization of synaptic vesicles of high purity. Brain Res 109, 285-309.

Nagy, G., Matti, U., Nehring, R. B., Binz, T., Rettig, J., Neher, E., and Sorensen, J. B. (2002). Protein kinase C-dependent phosphorylation of synaptosome-associated protein of $25 \mathrm{kDa}$ at Ser187 potentiates vesicle recruitment. J Neurosci 22, 9278-9286. 
Nagy, G., Reim, K., Matti, U., Brose, N., Binz, T., Rettig, J., Neher, E., and Sorensen, J. B. (2004). Regulation of Releasable Vesicle Pool Sizes by Protein Kinase A-Dependent Phosphorylation of SNAP-25. Neuron 41, 417-429.

Nicholls, D. G., and Sihra, T. S. (1986). Synaptosomes possess an exocytotic pool of glutamate. Nature 321, 772-773.

Nonet, M. L., Saifee, O., Zhao, H., Rand, J. B., and Wei, L. (1998). Synaptic transmission deficits in Caenorhabditis elegans synaptobrevin mutants. J Neurosci 18, 70-80.

Okabe, T., Sugimoto, N., and Matsuda, M. (1992). Calmodulin is involved in catecholamine secretion from digitonin-permeabilized bovine adrenal medullary chromaffin cells. Biochem Biophys Res Commun 186, 1006-1011.

Ossig, R., Schmitt, H. D., de Groot, B., Riedel, D., Keranen, S., Ronne, H., Grubmuller, H., and Jahn, R. (2000). Exocytosis requires asymmetry in the central layer of the SNARE complex. Embo J 19, 6000-6010.

Otto, H., Hanson, P. I., and Jahn, R. (1997). Assembly and disassembly of a ternary complex of synaptobrevin, syntaxin, and SNAP-25 in the membrane of synaptic vesicles. Proc Natl Acad Sci U S A 94, 6197-6201.

Oyler, G. A., Higgins, G. A., Hart, R. A., Battenberg, E., Billingsley, M., Bloom, F. E., and Wilson, M. C. (1989). The identification of a novel synaptosomal-associated protein, SNAP-25, differentially expressed by neuronal subpopulations. J Cell Biol 109, 30393052 .

Pallanck, L., Ordway, R. W., and Ganetzky, B. (1995). A Drosophila NSF mutant. Nature $376,25$.

Parlati, F., McNew, J. A., Fukuda, R., Miller, R., Sollner, T. H., and Rothman, J. E. (2000). Topological restriction of SNARE-dependent membrane fusion. Nature 407, 194198.

Paumet, F., Rahimian, V., and Rothman, J. (2004). The specificity of SNARE-dependent fusion is encoded in the SNARE motif. 101, -

Peduzzi, P., Holford, T., and Hardy, R. (1979). A computer program for life table regression analysis with time dependent covariates. Comput Programs Biomed 9, 106114.

Pennuto, M., Dunlap, D., Contestabile, A., Benfenati, F., and Valtorta, F. (2002). Fluorescence resonance energy transfer detection of synaptophysin I and vesicleassociated membrane protein 2 interactions during exocytosis from single live synapses. Mol Biol Cell 13, 2706-2717. 
Pevsner, J., Hsu, S. C., Braun, J. E., Calakos, N., Ting, A. E., Bennett, M. K., and Scheller, R. H. (1994). Specificity and regulation of a synaptic vesicle docking complex. Neuron 13, 353-361.

Pobbati, A. V., Razeto, A., Boddener, M., Becker, S., and Fasshauer, D. (2004). Structural basis for the inhibitory role of tomosyn in exocytosis. J Biol Chem 279, 47192-47200.

Quetglas, S., Iborra, C., Sasakawa, N., De Haro, L., Kumakura, K., Sato, K., Leveque, C., and Seagar, M. (2002). Calmodulin and lipid binding to synaptobrevin regulates calciumdependent exocytosis. Embo J 21, 3970-3979.

Quetglas, S., Leveque, C., Miquelis, R., Sato, K., and Seagar, M. (2000). Ca2+-dependent regulation of synaptic SNARE complex assembly via a calmodulin- and phospholipidbinding domain of synaptobrevin. Proc Natl Acad Sci U S A 97, 9695-9700.

Raptis, A., Torrejon-Escriban..., B., Gomez de Aranda, I., and Blasi, J. (2005). Distribution of synaptobrevin/VAMP 1 and 2 in rat brain. J Chem Neuroanat.

Reichert, V., and Moore, M. J. (2000). Better conditions for mammalian in vitro splicing provided by acetate and glutamate as potassium counterions. Nucleic Acids Res 28, 416423.

Reisinger, C., Yelamanchili, S. V., Hinz, B., Mitter, D., Becher, A., Bigalke, H., and Ahnert-Hilger, G. (2004). The synaptophysin/synaptobrevin complex dissociates independently of neuroexocytosis. J Neurochem $90,1-8$.

Rettig, J., and Neher, E. (2002). Emerging roles of presynaptic proteins in Ca++-triggered exocytosis. Science 298, 781-785.

Rice, L. M., Brennwald, P., and Brunger, A. T. (1997). Formation of a yeast SNARE complex is accompanied by significant structural changes. FEBS Lett 415, 49-55.

Richmond, J. E., Weimer, R. M., and Jorgensen, E. M. (2001). An open form of syntaxin bypasses the requirement for UNC-13 in vesicle priming. Nature 412, 338-341.

Rickman, C., Archer, D. A., Meunier, F. A., Craxton, M., Fukuda, M., Burgoyne, R. D., and Davletov, B. (2004). Synaptotagmin Interaction with the Syntaxin/SNAP-25 Dimer Is Mediated by an Evolutionarily Conserved Motif and Is Sensitive to Inositol Hexakisphosphate. J Biol Chem 279, 12574-12579.

Rickman, C., and Davletov, B. (2003). Mechanism of Calcium-independent Synaptotagmin Binding to Target SNAREs. J Biol Chem 278, 5501-5504.

Ridder, A. N. J. A., Morein, S., Stam, J. G., Kuhn, A., de Kruijff, B., and Killian, J. A. (2000). Analysis of the Role of Interfacial Tryptophan Residues in Controlling the Topology of Membrane Proteins. Biochemistry 39, 6521-6528. 
Bibliography

Rizo, J., and Sudhof, T. C. (2002). Snares and Munc18 in synaptic vesicle fusion. Nat Rev Neurosci 3, 641-653.

Rosenmund, C., Rettig, J., and Brose, N. (2003). Molecular mechanisms of active zone function. Current Opinion in Neurobiology 13, 509-519.

Roy, R., Laage, R., and Langosch, D. (2004). Synaptobrevin transmembrane domain dimerization-revisited. Biochemistry 43, 4964-4970.

Ruiz-Montasell, B., Aguado F, Majo G, Chapman ER, Canals JM, Marsal J, Blasi J. (1996). Differential distribution of syntaxin isoforms 1A and 1B in the rat central nervous system. Eur J Neurosci Dec;8(12), 2544-2552

Sakaba, T., Stein, A., Jahn, R., and Neher, E. (2005). Distinct kinetic changes in neurotransmitter release after SNARE protein cleavage. 309, -.

Sambrook, J., Russell, DW (2001). Molecular Cloning: A Laboratory Manual. ISBN: 0879695773

Sassa, T., Harada, S., Ogawa, H., Rand, J. B., Maruyama, I. N., and Hosono, R. (1999). Regulation of the UNC-18-Caenorhabditis elegans syntaxin complex by UNC-13. J Neurosci 19, 4772-4777.

Scales, S. J., Hesser, B. A., Masuda, E. S., and Scheller, R. H. (2002). Amisyn, a novel syntaxin-binding protein that may regulate SNARE complex assembly. J Biol Chem 277, 28271-28279.

Schiavo, G. G., Benfenati, F., Poulain, B., Rossetto, O., de Laureto, P. P., DasGupta, B. R., and Montecucco, C. (1992). Tetanus and botulinum-B neurotoxins block neurotransmitter release by proteolytic cleavage of synaptobrevin. Nature 359, 832-835.

Schoch, S., Deak, F., Konigstorfer, A., Mozhayeva, M., Sara, Y., Sudhof, T. C., and Kavalali, E. T. (2001). SNARE function analyzed in synaptobrevin/VAMP knockout mice. Science 294, 1117-1122.

Schuette, C. G., Hatsuzawa, K., Margittai, M., Stein, A., Riedel, D., Kuster, P., Konig, M., Seidel, C., and Jahn, R. (2004). Determinants of liposome fusion mediated by synaptic SNARE proteins. PNAS 101, 2858-2863.

Schulze, K. L., Broadie, K., Perin, M. S., and Bellen, H. J. (1995). Genetic and electrophysiological studies of drosophila syntaxin-1A demonstrate its role in nonneuronal secretion and neurotransmission. Cell 80, 311-320.

Sheng, M., and Kim, M. J. (2002). Postsynaptic Signaling and Plasticity Mechanisms. Science 298, 776-780. 
Bibliography

Shin, O. H., Rhee, J. S., Tang, J., Sugita, S., Rosenmund, C., and Sudhof, T. C. (2003). $\mathrm{Sr} 2+$ binding to the $\mathrm{Ca} 2+$ binding site of the synaptotagmin $1 \mathrm{C} 2 \mathrm{~B}$ domain triggers fast exocytosis without stimulating SNARE interactions. Neuron 37, 99-108.

SigmaAldrich

http://www.sigmaaldrich.com/sigma/general\%20information/detergent $\% 20$ selection $\% 20 \mathrm{~g}$ uide.pdf.

Sollner, T., Bennett, M. K., Whiteheart, S. W., Scheller, R. H., and Rothman, J. E. (1993a). A protein assembly-disassembly pathway in vitro that may correspond to sequential steps of synaptic vesicle docking, activation, and fusion. Cell 75, 409-418.

Sollner, T., Whiteheart, S. W., Brunner, M., Erdjument-Bromage, H., Geromanos, S., Tempst, P., and Rothman, J. E. (1993b). SNAP receptors implicated in vesicle targeting and fusion. Nature 362, 318-324.

Sorensen, J. B., Matti, U., Wei, S. H., Nehring, R. B., Voets, T., Ashery, U., Binz, T., Neher, E., and Rettig, J. (2002). The SNARE protein SNAP-25 is linked to fast calcium triggering of exocytosis. Proc Natl Acad Sci U S A 99, 1627-1632.

Sugita, S., Janz, R., and Sudhof, T. C. (1999). Synaptogyrins regulate Ca2+-dependent exocytosis in PC12 cells. J Biol Chem 274, 18893-18901.

Sutton, R. B., Fasshauer, D., Jahn, R., and Brunger, A. T. (1998). Crystal structure of a SNARE complex involved in synaptic exocytosis at 2.4 A resolution. Nature 395, 347353.

Takamori unpublished observations.

Teo, T. S., and Wang, J. H. (1973). Mechanism of activation of a cyclic adenosine 3':5'monophosphate phosphodiesterase from bovine heart by calcium ions. Identification of the protein activator as a Ca2+ binding protein. J Biol Chem 248, 5950-5955.

Tochio, H., Tsui, M. M., Banfield, D. K., and Zhang, M. (2001). An autoinhibitory mechanism for nonsyntaxin SNARE proteins revealed by the structure of Ykt6p. Science 293, 698-702.

Towbin, H., Staehelin, T., and Gordon, J. (1989). Immunoblotting in the clinical laboratory. J Clin Chem Clin Biochem 27, 495-501.

Trimble, W. S., Cowan, D. M., and Scheller, R. H. (1988). VAMP-1: A Synaptic VesicleAssociated Integral Membrane Protein. PNAS 85, 4538-4542.

Tucker, W. C., Weber, T., and Chapman, E. R. (2004). Reconstitution of Ca2+-Regulated Membrane Fusion by Synaptotagmin and SNAREs. Science 304, 435-438. 
Van Komen, J. S., Bai, X., Scott, B. L., and McNew, J. A. (2006). An intramolecular tSNARE complex functions in vivo without the syntaxin NH2-terminal regulatory domain. J Cell Biol 172, 295-307.

Verhage, M., Maia, A. S., Plomp, J. J., Brussaard, A. B., Heeroma, J. H., Vermeer, H., Toonen, R. F., Hammer, R. E., van den Berg, T. K., Missler, M., et al. (2000). Synaptic assembly of the brain in the absence of neurotransmitter secretion. Science 287, 864-869. Vilinsky, I., Stewart, B. A., Drummond, J., Robinson, I., and Deitcher, D. L. (2002). A Drosophila SNAP-25 null mutant reveals context-dependent redundancy with SNAP-24 in neurotransmission. Genetics 162, 259-271.

Walch-Solimena, C., Blasi, J., Edelmann, L., Chapman, E. R., von Mollard, G. F., and Jahn, R. (1995). The t-SNAREs syntaxin 1 and SNAP-25 are present on organelles that participate in synaptic vesicle recycling. J Cell Biol 128, 637-645.

Washbourne, P., Schiavo, G., and Montecucco, C. (1995). Vesicle-associated membrane protein-2 (synaptobrevin-2) forms a complex with synaptophysin. Biochem J 305 ( Pt 3), 721-724.

Washbourne, P., Thompson, P. M., Carta, M., Costa, E. T., Mathews, J. R., LopezBendito, G., Molnar, Z., Becher, M. W., Valenzuela, C. F., Partridge, L. D., and Wilson, M. C. (2002). Genetic ablation of the t-SNARE SNAP-25 distinguishes mechanisms of neuroexocytosis. Nat Neurosci 5, 19-26.

Weber, T., Zemelman, B. V., McNew, J. A., Westermann, B., Gmachl, M., Parlati, F., Sollner, T. H., and Rothman, J. E. (1998). SNAREpins: minimal machinery for membrane fusion. Cell 92, 759-772.

Wei, S., Xu, T., Ashery, U., Kollewe, A., Matti, U., Antonin, W., Rettig, J., and Neher, E. (2000). Exocytotic mechanism studied by truncated and zero layer mutants of the Cterminus of SNAP-25. Embo J 19, 1279-1289.

Whiteheart, S. W., Griff, I. C., Brunner, M., Clary, D. O., Mayer, T., Buhrow, S. A., and Rothman, J. E. (1993). SNAP family of NSF attachment proteins includes a brain-specific isoform. Nature 362, 353-355.

Widberg, C. H., Bryant, N. J., Girotti, M., Rea, S., and James, D. E. (2003). Tomosyn interacts with the t-SNAREs syntaxin 4 and SNAP23 and plays a role in insulin-stimulated GLUT4 translocation. J Biol Chem 278, 35093-35101.

Wiedenmann, B., and Franke, W. W. (1985). Identification and localization of synaptophysin, an integral membrane glycoprotein of $\mathrm{Mr} 38,000$ characteristic of presynaptic vesicles. Cell 41, 1017-1028. 
Willig, K. I., Rizzoli, S. O., Westphal, V., Jahn, R., and Hell, S. W. (2006). STED microscopy reveals that synaptotagmin remains clustered after synaptic vesicle exocytosis. Nature 440, 935-939.

Wimmer, C., Hohl, T. M., Hughes, C. A., Muller, S. A., Sollner, T. H., Engel, A., and Rothman, J. E. (2001). Molecular mass, stoichiometry, and assembly of $20 \mathrm{~S}$ particles. J Biol Chem 276, 29091-29097.

Xiao, W., Poirier, M. A., Bennett, M. K., and Shin, Y. K. (2001). The neuronal t-SNARE complex is a parallel four-helix bundle. Nat Struct Biol 8, 308-311.

Xu, T., Rammner, B., Margittai, M., Artalejo, A. R., Neher, E., and Jahn, R. (1999). Inhibition of SNARE complex assembly differentially affects kinetic components of exocytosis. Cell 99, 713-722.

Yelamanchili, S. V., Reisinger, C., Becher, A., Sikorra, S., Bigalke, H., Binz, T., and Ahnert-Hilger, G. (2005). The C-terminal transmembrane region of synaptobrevin binds synaptophysin from adult synaptic vesicles. Eur J Cell Biol 84, 467-475.

Yizhar, O., Matti, U., Melamed, R., Hagalili, Y., Bruns, D., Rettig, J., and Ashery, U. (2004). Tomosyn inhibits priming of large dense-core vesicles in a calcium-dependent manner. Proc Natl Acad Sci U S A 101, 2578-2583.

Zou, L. L., and Richardson, J. P. (1991). Enhancement of transcription termination factor rho activity with potassium glutamate. J Biol Chem 266, 10201-10209. 